A manual on guidelines for the control of arboviral encephalitides in Canada Agricuiture

Canada DEC 131990

Library / Bibliothèque, Ottawa K1A 0C5 
Digitized by the Internet Archive in 2013 


\section{A manual on guidelines for the control of arboviral encephalitides in Canada}

Submitted by

The Canada Biting Fly Centre

Department of Entomology

The University of Manitoba

Technical Bulletin 1990-5E

Research Branch

Agriculture Canada

1990 
Copies of this publication are available from Peter G. Mason

Secretar

Exper Committee of Insect Pests of Animals

Research Branch. Agriculture Canada

107 Science Crescent

Saskatoon. Saskatchewan

S7N 0X2

Produced by Research Program Service

c. Minister of Supply and Services Canada 1990

Cat. No. A54-8/1990-5E

ISBN $0-662-18197-2$

Contract DSS No. OSF85-00073

\section{Cover illustration}

The dots on the map represent

Agriculture Canada research

establishments. 


\section{PART I}

1. INTRODUCTION

PAGE

2. DISEASES

a. Western Equine Encephalitis

i. History

ii. Nature of Disease

iii. Clinical Aspects in Human Infections

iv. Epidemiology

b. St. Louis Encephalitis

i. History

ii. Nature of Disease

iii. Clinical Aspects in Human Infections

iv. Epidemiology

c. Eastern Equine Encephalitis

i. History

ii. Clinical Aspects

3. VECTORS

a. Known and Potential Vectors of Western Equine Encephalitis

i. Survey of Virus Isolations

1i. Virus Amplification

iii. Transmission

iv. Overwintering of Vectors

b. Known and Potential Vectors of Eastern Equine Encephalitis

c. Known and Potential Vectors of California Group Viruses

d. Possible Overwintering of Viruses

4. SURVEIILANCE

a. Mosquito Populations

i. Larval Surveys

ii. Adult Surveys

iii. Population Dynamics, Activity

iv. Interpretation of Trap Data 
i. Mosquito Populations $\quad 49$

ii. Sentinel Flocks 50

iii. Reservoirs, Indicators 51

c. Criteria 52

i. Mosquito Counts 52

ii. Significance of Seroconversion $\quad 54$

iii. Weather Factors $\quad 54$

d. Provincial Surveillance 55

i. Western Equine Encephalitis 55

ii. St. Louis Encephalitis 60

5. CRITERIA FOR CONTROL 61

a. Western Equine Encephalitis 61

b. St. Louis Encephalitis 68

6. CONTROL OPTIONS 69

a. Vector Suppression 69

i. Non-Chemical 70

ii. Use of Insecticides 71

iii. Aerial Insecticide Application 77

iv. Activity Periods of Vectors 80

v. Honey Bee Activity 81

b. Host Protection 83

i. Suitable Clothing 83

ii. Screening and Netting 83

iii. Behaviour Modification 84

iv. Topically Applied Repellents 85

v. Repellent-impregnated Clothing 86

vi. Personal Hygiene 87

vii. Human Vaccination 87

viii. Devices for Protection 87

ix. Horse Vaccination 87

$\mathrm{x}$. Repellents for Horses 88

$x i$. Smudges 88

xil. Insecticides 98

7. EMERGENCY VECTOR CONTROL 89

a. Emergency Vector Control by Municipalities 89

b. Value of Ground-Based Control 95

c. Assessment of Repellents 96

8. ENVIRONMENTAL MONITORING 101 
a. Spray Dispersal

b. Effects on Wildlife 103

c. Effects on Humans 105

$\begin{array}{ll}\text { 9. PUBLIC INFORMATION } & 117\end{array}$

$\begin{array}{ll}\text { a. Information Program } & 117\end{array}$

b. Media Option 120

$\begin{array}{ll}\text { c. Resource Materials } & 121\end{array}$

d. Specific Needs $\quad 124$

PART II

$\begin{array}{ll}\text { 1. SURVEILLANCE PROGRAM } & 127\end{array}$

a. Protocols for Arbovirus Surveillance 127

b. Resource Requirements for Surveillance Program

c. Components for Surveillance 128

i. Weather 128

ii. Mosquito Monitoring 128

iii. Virus Activity 130

iv. Equine Cases 132

v. Human Cases 133

vi. Contributions and Activities of Other Jurisdictions

d. Approach

2. CONTINGENCY PLANNING FOR IMPLEMENTATION OF EMERGENCY VECTOR CONTROL

a. General Approach to Aerial ULV Applications 135

b. Resource Requirements 136

$\begin{array}{ll}\text { c. Planning and Scheduling } & 141\end{array}$

d. Legislation and Jurisdiction 142

$\begin{array}{ll}\text { e. Procedural Guidelines } & 144\end{array}$

f. Reporting Procedures 147 
3. PUBLIC INFORMATION

a. Implementation 149

b. Maintenance Requirements 150

c. Assessment

\section{PART III}

REFERENCES CITED

APPENDIX

I. INVENTORY OF CHEMICALS REGISTERED IN CANADA

FOR MOSQUITO CONTROL AND AS REPELLENTS

II. INVENTORY OF EQUIPMENT USED IN CANADA FOR MOSQUITO CONTROL

III. SUPPLIERS OF REPELLENTS AND CHEMICALS

REGISTERED FOR MOSQUITO CONTROL IN CANADA

IV. ARBOVIRUS SURVEILLANCE PROGRAMS IN CANADA, 1980 
PART I 



\section{INTRODUCTION}

Mosquito-borne virus outbreaks in Canada have typically been sporadic and unpredictable. Consequently, when there is an outbreak, communities are caught by surprise and may be poorly prepared for the public health emergency to follow. The Canada Biting Fly Centre was contracted to help synthesize available information on mosquito-borne arboviruses in Canada, and to provide officials with the emergency measures necessary during an outbreak.

The contract was initiated by the Expert Committee of Insect Pests of Animals (Agriculture Canada), and expertise was drawn from the membership of The Expert Committee on Insect Pests of Animals. Contributing members of that Committee and other collaborators are listed below, followed by their sections of responsibility:

Dr. R.A. Brust - Dept. of Entomology, University of Manitoba, Winnipeg, Manitoba. R3T 2N2. Part I. Sections 3a(i, ii, iii, iv), 4a(iii, iv), 4b(i, ii), 4c(i,ii, iii), 6a(iv, v), $7 \mathrm{~b}$.

Dr. R.A. Costello - British Columbia Ministry of Agriculture and Fisheries, 1770-57th Ave., Surrey, British Columbia. V3S 124. Part I. Section 4a(i, ii).

Dr. R.A. Ellis - Prairie Pest Management, 207 Cullen Dr., Winnipeg, Manitoba. R3R 1P5. Part I. Sections 6a(i, ii, iii), 6b, 7a, c, 9a, b, c, d; Part II. 2, 3; Appendices I, II, and III.

Dr. M.M. Galloway, Director, Canada Biting Fly Centre, Dept.of Entomology, University of Manitoba, Winnipeg, Manitoba. R3T 2N2. Part I. Sections 4d(i), 5a; Part II, la, b, c, d; Appendix IV.

Dr. T.D. Galloway, Dept. of Entomology, University of Manitoba, Winnipeg, Manitoba. R3T 2N2. Part I. Sections 2a(i, ii, iii, iv), 3b, c, d, 4b(iii), 4d(ii), 5b.

Dr. W.L. Lockhart, Dept. of Fisheries and Oceans, Freshwater Institute, 501 University Crescent, Winnipeg, Manitoba. R3T 2N6. Part I. Sections $8 a, b, c$.

Dr. P.N. Nation, Head, Veterinary Pathology Branch, Alberta Agriculture, 6909116 St., Edmonton, Alberta. T6H 4P2. Part I. Section $2 a(i)$, Alberta data in Table 2 .

Dr. H.J. Smith, Food Production and Inspection Branch, Health of Directorate, Animal Pathology Division, Box 1410, 13 College St., Sackville, New Brunswick. EOA 3CO. Part I. Sections 2c(i, ii, iii).

Dr. G.A. Surgeoner, Dept. of Environmental Biology, University of Guelph, Guelph, Ontario. N1G 2Wl. Part I. Sections 2b(i, ii, iii, iv).

Funding for this project was provided through a contract from Agriculture Canada to The Canada Biting Fly Centre.

The support and financial assistance provided by Dean J.I.

Elliot and former Dean R.C. McGinnis of the Faculty of Agriculture, University of Manitoba, and the Office of Research 
Administration of the University of Manitoba are gratefully acknowledged. The assistance of Mr. R.M. Prentice, former Chair, and Dr. R. Trottier, Chair, of the Expert Committee of Insect Pests of Animals (Agriculture Canada) is also gratefully acknowledged. 


\section{INTRODUCTION}

Au Canada, les foyers de virus transmis par les moustiques ont toujours été sporadiques et imprévisibles. En conséquence, lorsqu'un foyer se déclare, les populations touchées sont prises au dépourvu et sont souvent mal préparées à faire face à la situation d'urgence sanitaire qui en découle. On a donc retenu les services du Centre canadien sur les insectes piqueurs pour participer à la synthèse de l'information disponible sur les arbovirus transmis par les moustiques au Canada et pour indiquer aux fonctionnaires les mesures à prendre dans de telles circonstances.

C'est le Comité d'experts des insectes nuisibles aux animaux (Agriculture Canada) qui a eu l'idée de ce contrat, et quelques-uns de ses experts ont permis de mener à bien ce projet. Vous trouverez ci-après les noms de ces membres et des autres collaborateurs, ainsi que leurs coordonnées et les sections de l'ouvrage qu'on leur a confiées.

Dr R.A. Brust - Département d'entomologie, Université du Manitoba, Winnipeg (Man.) R3T 2N2. Partie I. Sections 3a (i, ii, iii, iv), 4a (iii, iv), $4 \mathrm{~b}$ (i, ii), 4c (i, ii, iii), $6 \mathrm{a}$ (iv, v), $7 \mathrm{~b}$.

$\mathrm{D}^{\mathrm{r}}$ R.A. Costello - British Columbia Ministry of Agriculture and Fisheries, 1770-57th avenue, Surrey (C.-B.) V3S 124. Partie I. Section 4a (i, ii).

Dr R.A. Ellis - Prairie Pest Management, 207 Cullen Dr., Winnipeg (Man.) R3R 1P5. Partie I. Sections 6a (i, ii, iii), 6b, 7a, c, 9a, b, c, d; Partie II. 2, 3; Annexes I, II et III.

$D^{\mathrm{r}}$ M.M. Galloway, directeur, Centre canadien sur les insectes piqueurs, Département d'entomologie, Université du Manitoba, Winnipeg (Man.) R3T 2N2. Partie I. Sections 4d (i), 5a; Partie II, la, b, c, d; Annexe IV.

Dr T.D. Galloway, Département d'entomologie, Université du Manitoba, Winnipeg (Man.) R3T 2N2. Partie I. Sections $2 a$ (i, ii, iii, iv), $3 b, c, d, 4 b$ (iii), 4d (ii), 5b.

$\mathrm{D}^{\mathrm{r}}$ W.L. Lockhart, ministère des Pêches et des Océans, Institut des eaux douces, 501 University Crescent, Winnipeg (Man.) R3T 2N6. Partie I. Sections $8 \mathrm{a}, \mathrm{b}, \mathrm{c}$.

$D^{r}$ P.N. Nation, Head, Veterinary Pathology Branch, Alberta Agriculture, 6909116 St., Edmonton (Alb.) T6H 4P2. Partie I. Sections 2a (i), données concernant 1 'Alberta au tableau 2 . 
${ }^{\mathrm{r}} \mathrm{H} . J$. Smith, Direction générale de 1 a production et de l'inspection des aliments, Direction de l'hygiène vétérinaire, Division de la pathologie animale, Case postale 1410, 13, rue College, Sackville (N.-B.) EOA 3CO. Partie I. Sections 2c (i, ii, iii).

$D^{r}$ G.A. Surgeoner, Dept. of Environmental Biology, University of Guelph, Guelph (Ont.) N1G 2Wl. Partie I. Sections 2b (i, ii, iii, iv).

Le projet en question a été financé dans le cadre d'un contrat qu'Agriculture Canada a passé avec le Centre canadien sur les insectes piqueurs.

Nous témoignons notre reconnaissance au doyen actuel de la Faculté de l'agriculture de l'Université du Manitoba, M. J.I. Elliot et à son prédécesseur, M. R.C. McGinnis, ainsi qu'au Bureau de l'administration de la recherche de 1'Université du Manitoba pour leur soutien et leur aide financière. Nous remercions aussi le $D^{r}$. Trottier, président du Comité d'experts des insectes nuisibles aux animaux (Agriculture Canada) et son prédécesseur, M. R.M. Prentice, de leur précieuse contribution. 



\section{DISEASES}

a. Western Equine Encephalitis

1. History

The early recognition of WEE virus as a disease-causing agent was understandably difficult. Clinical symptoms are not glaringly unique and most often occur in horses and humans. Association of a common causative agent in these two diverse hosts was not easily accommodated. Disease outbreaks have historically been sporadic, interspersed by periods of virtual absence of clinical activity. Detection and isolation of virus required special handling of affected tissues. Epidemics and epizootics have not always coincided closely. Consequently, the first isolation of WEE virus by Meyer et al. (1931) from horses, and confirmation of the relationship of this same virus to human encephalitis by Howitt (1938) were landmarks in an understanding of this disease.

The WEE virus is apparently widespread in Canada and human cases have been reported from British Columbia, Alberta, Saskatchewan, Manitoba and Quebec (Artsob and Spence 1979, Table 1). However, the Prairie Provinces have been the prime focus for both human and equine infections. For the total number of 1607 confirmed human cases reported from Canada, 865 (54\%) and 674 (42\%) occurred in Saskatchewan and Manitoba, respectively. Over $68 \%$ (1094 cases) of all human cases were recorded during a single epidemic in the prairies in 1941 (Table 1).

Epizootics of WEE in horses were recognized in Canada for many years before the causative agent was isolated and identified in 1935 by Fulton (1938). Previous to 1935, it was known that horses suffered from an affliction of the nervous system, but were diagnosed in somewhat vague terms as having forage poisoning, botulism, cerebro-spinal meningitis, corn-stalk disease, sleeping sickness or blind staggers (Fulton 1938; Artsob and Spence 1979). As for WEE in humans, cases of WEE in horses are widespread in Canada, reported from British Columbia, Alberta, Saskatchewan, Manitoba and northwestern Ontario (Table 2; Artsob and Spence 1979). Many thousands of horses were lost to WEE in Saskatchewan and Manitoba, again the provinces where clinical disease in horses has been most prevalent. The development of an effective vaccine for horses, introduced in 1938, seems to have limited the relative magnitude of epizootics. For example, it was estimated that $9 \%$ of the Manitoba horse population was clinically affected in the outbreak of 1937 (data collected by questionnaire circulated to 70 municipalities) (Savage 1942). Since the widespread availability of the WEE vaccine, the incidence of WEE has not approached even $1 \%$ of the total horse population.

It is fair to assume that Public Health personnel and veterinarians will face isolated cases and outbreaks in the future. Increased awareness of WEE in recent years should result in rapid diagnosis and optimal treatment in the majority of cases. 
1i. Nature of Disease

Western Equine Encephalitis virus is an Alphavirus in the

Togaviridae. There are apparently three serologically similar variants of WEE virus, only one of which, WEE virus sensu stricta, will be considered here. The other two are Highlands J virus and Fort Morgan virus. Highlands $\mathrm{J}$ virus was once thought to be a less virulent strain of WEE virus, found in the eastern U.S. and Gulf Coast states, and is in many ways ecologically reminiscent of WEE virus (see Hayes and Wallis 1977 for a review). Fort Morgan virus has so far been recovered only from the cliff swallow - English sparrow - swallow bug system in the west central U.S. (Calisher et al. 1980).

WEE virus is widespread in the New World. It is most prevalent and best known in the western and south central U.S. and Prairie Provinces, where there have been numerous outbreaks in humans and horse populations since recognition of the disease. However, the virus is also prevalent in South America, and outbreaks are known in Brazil, Guyana, Uraguay and Argentina (Grimstad 1983).

The enzootic cycle of WEE virus involves mosquitoes as primary vectors and wild avian hosts. This cycle has been studied in greatest detail in foci in California and Texas where Culex tarsalis is the most important vector for the virus. Nestling passerines are the principal amplfying factor for the virus, and horizontal transfer continues throughout the bird population as long as there are sufficient susceptible hosts and vector mosquitoes.

Epizootics and epidemics are initiated when the prevalence of virus in the endemic cycle increases, and probability of horses and humans contacting infected vectors is high. Clinical symptoms in horses and humans may or may not be present, but the duration and level of viremia are insufficient to warrant their contributing significantly to the amplication or persistence of the virus. Unfortunately, the consequences of clinical WEE in these cases can be serious, resulting in death or permanent physical and behavioural disability.

Involvement of humans and horses in the WEE disease cycle has prompted a great deal of intensive research activity. However, one aspect of the disease remains enigmatic and offers room for considerable speculation. There is still no convincing evidence for the support of an overwintering mechanism.

Transovarial transmission in mosquitoes, mites and bird is apparently negative. The virus may be capable of overwintering in adult female mosquitoes, but evidence so far is weak. Vectors

other than Culex tarsalis may be important, perhaps Culiseta spp., Anopheles spp. or even spring Aedes spp., but their role is unclear. It has been suggested that hibernating vertebrates may be able to harbour the virus through the winter and display a high or cyclic viremia when they resume activity in the spring. Snakes, frogs, turtles, and ground squirrels have all been considered possible candidates, but there is no clear support from 
field data. Migratory birds may carry chronic latent infections and display a viremia at the onset of reproductive activity, or may become infected in the south and carry a virus infection with them on their northward flight. Again, however, these mechanisms are speculative in regard to the establishment of new disease foci or generation of epidemic cycles. It has also recently been suggested that infected mosquitoes may be borne considerable distances on prevailing winds from endemic foci into non-endemic regions. Clearly, the question of overwintering of WEE virus should provide a significant objective for future research in the field.

In Canada, epidemics have been sporadic and rarely involved large numbers of people. Even though consequences of clinical disease can be severe, it is difficult to justify continued expenditure of public funds in support of the long term field and laboratory research necessary before we can gain a good understanding of the epidemiology of WEE. With the inevitable occurrence of epidemics in the future, it seems unlikely that researchers and medical personnel will be able to provide the answers to questions asked by the public and political decision makers.

\section{1i1. Clinical Aspects in Human Infections}

Humans and horses play no major role in the epidemiology of WEE virus. However, because of the severe clinical manifestations of disease in these dead-end hosts, and the frequency and magnitude of outbreaks in the Prairie Provinces (see Tables 1, 2), WEE must be considered, historically, the most important mosquitoborne virus in Canada. During epidemics of WEE, there has been ample opportunity to document closely, the onset and development of symptoms, pattern of recovery, sequelae, and pathology.

The onset of clinical symptoms of WEE may be gradual, increasing in intensity or may be sudden, especially in young children and infants. In most cases, acute illness is preceeded by headache, fever, drowsiness and gastrointestinal disturbance (Pavilanis et a1. 1957; Friesen and Eadie 1982). With the exception of headache, these symptoms are generally mild and cause for little concern in patients. The intense and persistent headache may last for several days, prompting the person to seek medical attention. Upon examination, the patient displays symptoms more closely associated with flu virus infection than central nervous system involvement (Adamson and Dubo 1942).

The acute phase of WEE presents an array of non-specific central nervous system disorders, and may last, on average, three weeks (Gareau 1941). Headache may persist, in a high percentage of cases, accompanied by a variably high fever running from 38.4 40.4 $\mathrm{C}$ (Waters 1976). The febrile period may last from 7-10 days before returning to normal. Convulsions and delerium commonly accompany periods of high fever, and may not necessarily be associated with direct damage to the brain by virus infection. Convulsions more commonly seem to accompany infections in infants (Adamson and Dubo 1942; Medovy 1943). 
Cerebrospinal fluid may be under pressure infrequently or for short duration, and is rarely severe. All counts are generally elevated and may persist in such condition throughout the acute phase. The cell content is dominated by polymorpholeucocytes early in the infection, gradually falling to normal after about 4 days (Adamson and Dubo 1942). Mononuclear cells generally predominate after 3-4 days. Total cell counts reach their highest levels during the first few days (averaging 150; as high as 400 or more) (Adamson and Dubo 1942), and remain elevated for at least 9-10 days.

There are a variety of non-specific associated symptoms during the acute phase. Most patients experience some degree of sleep disturbance, predominately somnolence, though examples of insomnia are not uncommon. There may be a degree of muscle pain or stiffness and like stiffness of the spine, is generally not severe, and not of great concern to the patient. Muscle discomfort apparently lasts only 4-5 days during the acute phase (Adamson and Dubo 1942). Medovy (1943) reported that, in infants, a peculiar rigidity, which was accompanied by high fever and general restlessness, was one of the earliest symptoms observed and lasted the longest.

Patients are frequently anorexic, and experience nausea and abdominal cramps (Gareau 1941). Vomiting is prevalent among patients but is not usually frequent or peristent. Those people who suffer vomiting generally do so for only one day. Bladder and bowel disorders are common and constipation may require frequent attention and treatment.

Tremors, characteristically in the facial muscles, lips and tongue are frequently reported and Adamson and Dubo (1942) considered these signs valuable in diagnosing WEE as opposed to polio during the 1941 epidemics. Some degree of speech impediment occurs, largely due to the disfunction of facial and throat muscles, and speech may be slurred or very slow. The face in general may take on a flushed or masked appearance or have an overall puffiness. The nose may be red, and the eyes inflamed. Sweating during this phase may be profuse and patients frequently experience chills as well (Adamson and Dubo 1942; Friesen and Eadie 1982).

In severe cases, patients may suffer a distinct loss of motor control and coordination (Friesen and Eadie 1982).

Paralysis is not frequent and is not usually severe or widespread. Patients commonly exhibit at least dulling of the senses or mental confusion to a general stupor. In the extreme, 10-20\% of clinical cases, patients may become comatose for variable periods of time, often up to 3 or 4 days (Adamson and Dubo 1942; Friesen and Eadie 1982). One of the remarkable clinical features of WEE is that a person may suffer from this extended period of coma, and still recover fully.

Unfortunately, the various signs and symptoms described above appear to be of little prognostic value. Death has occurred in approximately $10 \%$ of the reported clinical cases in Canada (see Table 1). Highest mortality rates seem to be associated with 
cases involving infants (less than 1 year old at the time of infection), and the elderly, often following respiratory or gastrointestinal complications.

Patients may recover fully following the acute phase of the infection, or exhibit varying degree of sequelae. Most studies have included examination of patients within a year of release from hospital. In these studies, a high proportion of patients still suffer from residue such as subtle changes in mood or personality, fatigue, slight tremors or twitches, slight loss of fine and/or gross motor control, inability to concentrate, sleep/rest disorders, or sensory distortion, involving hearing, speech or taste (Mulder et al. 1952; Friesen and Eadie). Occasionally, there may be severe psychological disturbance that may restrict normal social interaction or necessitate institutionalization (Fulton and Burton 1953; Earnst et al. 1971). Neurologic sequelae including convulsions, spastic weakness or brain disfunction may also be apparent (Earnst et al. 1971). Mulder et al. (1952) and Hawryluk et al. (1982) felt that severity of sequelae, at least among adult patients, could be directly correlated with duration of the comatose period or degree of debility during the acute phase of the disease.

In summary, WEE is a virus disease characterized by a complicated and variable pattern of onset and acute phase. Accurate confirmation of diagnosis cannot usually be made on the basis of the clinical picture, and medical authorities must rely on a serological diagnosis. Recovery may be rapid and complete, but in some patients sequelae occur which may be mild to severe. The recovery period often requires more than a year, and in cases where permanent damage has occurred patients never fully recover.

Pathology in humans following WEE virus infection seems largely to be restricted to the brain and central nervous system. At the time of autopsy, the organs appear generally unremarkable, or perhaps slightly oedematous (Quong 1942). There is no gross haemorrhage associated with brain tissues and there are no obvious diagnostic characteristics.

Quong (1942) identified three categories of microscopic change associated with the nerve tissues following post mortem examination on 18 human cases during the 1941 epidemic in Manitoba. The leptomeninges were oedematous and separated, containing scattered lymphocytes and glial cells. Perivascular cuffing, also observed by Rozdilsky et al. (1968), was evident, with lymphocytes and glial cells predominating. Finally, there were numerous small (0.2-0.4 mm), scattered areas of cell breakdown and deterioration. These lacunae eventually become filled with small cells, but were often seen as clear areas in histological preparations and gave the tissues what Quong (1942) referred to as a moth-eaten appearance. He even suggested that because the areas of damage were so small and scattered, that this may account for the relative freedom of serious sequelae in the majority of humans who recovered from the acute phase of the disease. Quong (1942) found that the grey matter was most seriously affected, while Rozdilsky et al. (1968) observed similar 
tissue destruction more extensively in the white matter.

Pathology, therefore, is quite restricted to the central nervous tissues. However, Monath et al. (1978) discovered that they could produce myocarditis and impaired cardiac function in mice infected with WEE virus. To date, there is no evidence to support similar cardiac involvement in humans.

\section{iv. Epidemiology}

By the very nature of Western Equine Encephalitis outbreaks in humans in Canada, it is difficult to assemble a meaningful epidemiological picture. The epidemics are sporadic and often do not encompass large numbers of clinical human cases (see Table 1). The majority of the data summarized below were collected during the outbreaks in the Prairie Provinces in 1941 (1094 cases), 1975 (14 cases) and 1981 (25 cases).

\section{The 1941 Epidemic}

Introduction

By virtue of the relative magnitude of the 1941 epidemic, we can gain better understanding of the epidemiology of WEE as it affects humans, than in any other epidemic period in Canada. The data collected during 1941 were reported for Alberta by McGugan (1942), for Saskatchewan by Davison (1942), and for Manitoba by Donovan and Bowman $(1942 a, b)$ and most of the following discussion is drawn from these sources. Coincident with the WEE epidemic, the Dominion Bureau of Statistics conducted their eighth national census on 2 June, 1941, and was the source of demographic information (Vo1. I 1950; Vo1 III 1946).

Canada was in a period of turmoil in 1941. The country had passed through a period of severe economic and industrial depression. On the prairies, there was a reduced farm population and the numbers of marriages and births had declined. For the first time in Canada's history, the population below age 10 , failed to exceed the 10-19 age class size. Immigration into Canada was reduced and industrial centres were faced with acute housing shortages.

By 1941, the economy showed signs of improvement, but Canada was at war, and approximately 313,452 males were enlisted in the Armed Forces at the time of the census in June. As a consequence of the depleted young male population and the increasing demand for food and supplies, changes took place in the previous employment pattern. More women were drawn into the active work force, and the average age of farm operators increased. The training activity of Armed Forces personnel resulted in widespread movement of troops, many of which came to bases on the Prairies, in rapidly prepared camp and barrack environments where men were concentrated in high densities.

Medical personnel in the Prairies were on the alert as the summer of 1941 unravelled. What was described as a major poliomyelitis epidemic in Manitoba and Alberta, began in June, and continued until early November, with 966 reported cases. Many of the early WEE cases were initially diagnosed as poliomyelitis. 
The temporal course of the WEE epidemic in the summer of 1941 was similar among the three provinces involved.

Saskatchewan, with 543 cases, reported the greatest spread from first to last dates of onset. One case in April, one in May and additional cases in June all occurred before any clinical activity was observed in either Alberta or Manitoba. No onset of WEE was reported from the latter provinces until late July. The peak in numbers of cases reported was uniformly in mid to late August. In Manitoba and Saskatchewan 195 (38\%) and 180 (33\%) of the cases were reported in weeks ending 19 and 23 August, respectively. By autumn, the incidence of WEE declined, until no further human cases occurred beyond 10 September, 14 October and 15 November for Alberta, Manitoba and Saskatchewan, respectively.

The data on numbers of clinical cases of WEE in humans, according to age and sex, were not reported in consistent fashion among provinces. Consequently, these data are summarized here (Table 3 ) according to three quite broad age categories i.e. infants ( $<1$ year of age), adolescents (ages 1-20) and adults $(>20$ years of age). For Alberta, McGugan (1942) provided no information on sexes by age of patients, other than that of the total, 30 (71.4\%) were males and 12 (29.6\%) were females. Data for Manitoba and Saskatchewan were more complete, but authors used different age groupings in reporting numbers of cases.

\section{Geographic Distribution}

As pointed out by Donovan and Bowman $(1942 a, b)$ there was no real spread of WEE in the 1941 epidemic, in the sense that human cases did not radiate out from isolated foci. Rather, cases were reported in seemingly random fashion from throughout the affected area.

Most cases of WEE were, however, from the southern region of the Prairie Provinces. For example, approximately $80 \%$ of the human cases in Manitoba occurred south of $50^{\circ} \mathrm{N}$ latitude, and about $72 \%$ in Saskatchewan were in south central areas of the province, south of Regina. In Alberta, $75 \%$ were reported from the southeastern corner of the province. In Manitoba cases were reported from as far north as the Swan River area in the western region, and Fisher Branch and Chattfield areas in the Interlake. In Saskatchewan, there was one case reported from the Meadow Lake area, along with 7 cases from Saskatoon.

There was a rural bias in the reported human cases in the 1941 epidemic. Approximately $80 \%$ of the Manitoba cases were from outside the most densely populated urban areas. Despite half the population of the province residing in Winnipeg and suburbs, less than $1 / 3$ (166 cases) of the cases occurred there. Similarly in Saskatchewan nearly $2 / 3$ of the total cases were from rural areas. As one would assume from these statistics, the outdoor workers seemed to be at greatest risk of exposure. In Alberta $>90 \%$ of the clinical cases were farm family members (Davison 1942; Donovan and Bowman 1942a,b; McGugan 1942). 
Age Distribution and Sex Ratios

Very little can be deduced from the WEE epidemic in Alberta in 1941. Only 42 total human cases were reported, and these only in the 1-19 year and > 19 year age classes. No infants developed clinical symptoms, and number of clinical cases in the older age classes did not exceed $0.1 / 1000$ (0.07 and 0.04 for the 1.19 and $>$ 19 year age classes respectively).

The population of Saskatchewan exceeded that in Manitoba for all age classes (Table 4), but the proportions of each sex were approximately equal. In both provinces, infants most often exhibited clinical symptoms (2.7 and 2.1/1000 for Saskatchewan and Manitoba respectively) although these data did not necessarily correlate with the proportion of deaths (Table 3). Adolescents were least affected in terms of manifested clinical signs, with only 0.4 and $0.3 / 1000$ for Saskatchewan and Manitoba respectively. In Saskatchewan proportionally fewer people in the adult age class became ill (0.7/1000), compared to Manitoba (0.9/1000). However in both provinces over $30 \%$ of the total reported WEE cases occurred in the over 45 age classes, where only about $20 \%$ of the total population was included.

The proportion of clinical cases among infants was approximately equal for males and females in both Saskatchewan and Manitoba (Table 3). In the older age classes, where sex specific occupational differences are expected to be more pronounced, the proportion of cases in males greatly exceeded that among females (Table 3). For Saskatchewan and Manitoba combined, nearly $69 \%$ of the total cases in the adolescent and adult age classes were male.

Occupational Relationships

No quantitative analysis of occupation for each of the reported WEE cases was published for the 1941 epidemic. McGugan (1942) did determine that "slightly over ninety per cent of all reported cases occurred among farmers and farmers' families". Davison (1942) suggested that because the peak of pathogen transmission occurred during the summer holiday season, many of the urban infections may have been acquired during visits to rural or recreational areas.

\section{The 1975 Epidemic}

Introduction

By comparison to the widespread 1941 epidemic of WEE in the Canadian Prairie Provinces, subsequent disease activity among human populations in Canada has been insignificant. However, epidemics in Manitoba in 1975 and again in 1981 were intensively studied and co-ordinated efforts were made to maximize the amount of information gathered. In 1975, there were only 14 confirmed human cases, all in Manitoba (Waters 1976). The earliest confirmed case was reported during the first week of August, and the last after 6 September. The largest number of cases (6) for any week occurred during the period 23-29 August. No deaths were attributed to WEE virus in 1975. Because of the small number of 
cases, conclusions regarding peak activity, age and sex class affected, and geographic pattern are limited, but are summarized here as for the 1941 outbreak.

\section{Geographic Distribution}

Seven cases were reported in patients who lived in Winnipeg or on the outskirts. The remainder were distributed throughout southern Manitoba, the most northerly human case from Gimli.

Age Distribution and Sex Ratio

All age groups were affected in the 1975 outbreak. Two cases, both males, occurred in infants; six patients ( 3 males; 3 females) were adolescents; six ( 4 males; 2 females) were adults. There was an overall preponderance of cases among males ( 9 vs. 5 females), but the small number of cases makes conclusions questionable.

Occupational Relationships

No data were reported on the occupation of the various patients during 1975.

The 1981 Epidemic

Introduction

In 1981, the only clinical human WEE cases reported in Canada occurred in Manitoba and were described in great detail by Friesen and Eadie (1982), Hawryluk et al. (1982), and Eadie and Friesen (1982). Of the 25 human cases in 1981, 22 were interviewed in person or by telephone, and the additional information gained from relatives, friends, neighbours and colleagues (Eadie and Friesen 1982), to produce the most complete epidemiological profile of any Canadian WEE outbreak. As in 1975, the total number of cases (25), with two deaths, does not allow extensive analysis.

The first case occurred on $13 \mathrm{July}$, and numerous cases were reported sporadically until the last onset on 10 October (Sekla and Stackiw 1982). The greatest number of cases was recognized during the period 10-24 August ( 9 cases), but three cases per week occurred during the other weeks of August.

\section{Geographic Distribution}

Twelve of the total human cases were either from Winnipeg ( 5 cases) or other urban Manitoba areas ( 7 cases). The remaining 13 were from rural areas. There were no apparent foci of infection, all cases being widely distributed. The northernmost case was just south-west of Swan River. There were no cases in either the Interlake Region, or in the eastern boreal forest zone of the province.

Age Distribution and Sex Ratio

There was one male infant case and two male adolescents cases in 1981. The remaining cases were 18 adult males, and 4 adult females. There was a preponderance of male cases in all age 
classes.

Occupational Relationships

Farm workers were a dominant component of the clinical cases in 1981. Ten patients were defined as full time farmers, and on average spent more time outdoors than the average for all other patients. Three additional cases were part-time farmers. The remaining cases were distributed among various occupations.

\section{Conclusions}

The outbreaks of clinical WEE in humans in Canada have been sporadic, and with the exceptions of 1941, 1975 and 1981, less than extensively reported. Among these three years, the 1941 data alone contains enough cases from which we can draw conclusions, but trends in both 1975 and 1981 in Manitoba are supportive.

With the exception of a few very early and very late cases seen in 1941 in Saskatchewan, it seems one can expect the first cases to appear in the latter part of July, with peak clinical activity in August. The rate of appearance of new cases would be expected to subside in September, with perhaps a few late cases in October. At the moment there is no available means to predict either number or location of cases. Since a large proportion of the population is susceptible to WEE virus, presumably exposure to the pathogen is related to probability of contact with infected mosquito vectors. Although a greater proportion of reported clinical cases are males, presumably this bias is a reflection of a greater probability of contact with vectors, rather than some intrinsic factor. Factors affecting manifestation of disease symptoms are unknown, but infants seem to be particularly sensitive to infection. 
Table 1. Numbers of human clinical cases of WEE reported for each province in Canada, 1935-1986. There were no cases reported in years not cited.

Province Year No. of Cases No. of Deaths

British Columbia $\quad 1971$

Alberta

Saskatchewan

1963

$1935-7$

1938

1939

1941

1947

1953

1963

1964

1965

1966

1967

1969

Manitoba

1938

1941

1947

1975

1977

1981

1983
1941
$14^{5}$

42

6

present
29
few
543
68
53

$90-100$

1

72

2

1

1

27
509
81
14
5
25
18

$0^{2^{1}}$

$8^{3}$

$0^{4}$

N.A. ${ }^{5}$

N.A. ${ }^{7}$

N.A. ${ }^{8}$

$3^{7}$

$0^{9}$

$8^{10}$

$0^{9}$

$0^{9}$

$0^{9}$

$6^{11,12}$
$78^{11,12,13}$
N.A..$^{14}$
$0^{15}$
$0^{16}$
$2^{16}$
$1^{17}$

Ontario

Quebec

1955

$0^{18}$

${ }^{1}$ Kettyls et al.(1972); ${ }^{2}$ Kettyls and Bowmer (1975); ${ }^{3}$ McGugan (1942); ${ }^{4}$ Morgante et al. (1968); ${ }^{5}$ Fulton (1941); ${ }^{6}$ Davison (1942); ${ }^{7}$ Dillenberg (1965); ${ }^{8}$ Dillenberg et al. (1956); ${ }^{9}$ McLintock et al. (1979); ${ }^{10}$ Rozdilsky et al. (1968); ${ }^{11}$ Donovan and Bowman (1942a); ${ }^{12}$ Donovan and Bowman (1942b); ${ }^{13} \mathrm{Jackson}(1942) ;{ }^{14} \mathrm{Sne} 11$ (1966); ${ }^{15}$ Sekla and Stackiw (1976); ${ }^{16}$ Sekla and Stackiw (1982); ${ }^{17}$ Sekla (unpublished); ${ }^{18}$ Pavilanis et al. (1957). 
Table 2. Numbers of confirmed WEE equine cases reported for each province in Canada, 1935-1986. There were no cases confirmed in years not cited.

\begin{tabular}{|c|c|c|c|}
\hline Province & Year & No. of Cases & No. of Deaths \\
\hline \multirow[t]{3}{*}{ British Columbia } & 1971 & 60 & $15^{1}$ \\
\hline & 1972 & 17 & N.A. ${ }^{2}$ \\
\hline & 1973 & 7 & N.A. ${ }^{2}$ \\
\hline \multirow[t]{6}{*}{ Alberta } & 1965 & 136 & N.A. ${ }^{3}$ \\
\hline & 1977 & & 420 \\
\hline & 1980 & & 120 \\
\hline & 1981 & & 1720 \\
\hline & 1982 & & $1^{20}$ \\
\hline & 1983 & & $1^{20}$ \\
\hline \multirow[t]{18}{*}{ Saskatchewan } & 1935 & extensive & N.A. ${ }^{4}$ \\
\hline & 1937 & extensive & N.A. ${ }^{4}$ \\
\hline & 1938 & $-52,500$ & $>15,000^{5}$ \\
\hline & 1941 & present & N.A. ${ }^{6}$ \\
\hline & $1953-4$ & 76 & N.A. ${ }^{7}$ \\
\hline & 1962 & extensive & N.A. ${ }^{8,9}$ \\
\hline & 1963 & 279 & 479 \\
\hline & 1964 & 41 & N.A. ${ }^{10}$ \\
\hline & 1965 & 106 & N.A. ${ }^{10}$ \\
\hline & 1966 & 8 & N.A. ${ }^{10}$ \\
\hline & 1967 & 10 & N.A. ${ }^{10}$ \\
\hline & 1968 & 9 & N.A. ${ }^{10}$ \\
\hline & 1969 & 40 & N.A. ${ }^{10}$ \\
\hline & 1970 & 17 & N.A. ${ }^{10}$ \\
\hline & 1971 & 7 & N.A. ${ }^{10}$ \\
\hline & 1974 & 4 & N.A. ${ }^{10}$ \\
\hline & 1975 & 23 & N.A. ${ }^{10}$ \\
\hline & 1981 & 2 & N.A. ${ }^{10}$ \\
\hline \multirow[t]{13}{*}{ Manitoba } & 1935 & extensive & N.A. ${ }^{4}$ \\
\hline & 1937 & $-12,000$ & $>2,000^{12}$ \\
\hline & 1938 & extensive & N.A. ${ }^{12}$ \\
\hline & 1941 & present & N.A. ${ }^{6}$ \\
\hline & 1963 & 173 & N.A. ${ }^{13}$ \\
\hline & 1964 & 73 & N.A. ${ }^{13}$ \\
\hline & 1965 & 75 & $9^{14}$ \\
\hline & 1966 & 51 & N.A. ${ }^{15}$ \\
\hline & 1968 & 14 & N.A. ${ }^{13}$ \\
\hline & 1969 & 41 & N.A. ${ }^{13}$ \\
\hline & 1975 & 145 & N.A. ${ }^{13,16}$ \\
\hline & 1976 & 10 & N.A. ${ }^{16}$ \\
\hline & 1977 & 53 & N.A. ${ }^{16}$ \\
\hline
\end{tabular}


Province Year No. of Cases No.of deaths

\begin{tabular}{lrrr}
\hline 1978 & 1 & N.A. ${ }^{16}$ \\
1980 & 1 & N.A. ${ }^{16}$ \\
1981 & 120 & N.A. ${ }^{17}$ \\
1982 & 1 & N.A. ${ }^{17}$ \\
1983 & 23 & N.A. \\
Ontario & & & $1{ }^{18}$ \\
& 1937 & 1 & N.A. ${ }^{13}$ \\
& 1975 & 4 & N.A. ${ }^{19}$ \\
\hline
\end{tabular}

Kettyls et al. (1972); ${ }^{2}$ Kettyls and Bowmer (1975); ${ }^{3}$ Morgante et al. (1968); ${ }^{4}$ Fulton (1938); ${ }^{5}$ Davison (1942); ${ }^{6}$ Cameron (1942); ${ }^{7}$ Dillenberg et al. (1956); ${ }^{8}$ Spalatin et al. (1963); ${ }^{9} \mathrm{Dillenberg}(1965)$; ${ }^{10} \mathrm{Spalatin}$ et al. (1963); ${ }^{9} \mathrm{Dillenberg} \mathrm{(1965);}$ ${ }^{10}$ McLintock et al. (1979); ${ }^{11}$ Neufeld and Nayar (1982); ${ }^{12}$ Savage (1942); ${ }^{13}$ Lillie et al. (1976); ${ }^{14}$ Snell (1966); ${ }^{15}$ McKay et al. (1968); ${ }^{16}$ Sekla and Stackiw (1982); ${ }^{17}$ Sekla (unpublished); ${ }^{18} \mathrm{Mitche} 11$ and Walker (1941); ${ }^{19}$ Surgeoner et al. (1982); ${ }^{20}$ Nation (unpublished). 
Table 3. Summary of numbers of clinical human case and deaths by age and sex in the Western Equine Encephalitis epidemic in the Canadian Prairie Provinces, 1941.

\section{Alberta ${ }^{1}$ \\ Saskatchewan ${ }^{2}$ \\ Manitoba ${ }^{2}$}

\begin{tabular}{|c|c|c|c|c|c|c|}
\hline $\begin{array}{l}\text { Age/ } \\
\text { Class }\end{array}$ & $\begin{array}{l}\text { No. of } \\
\text { Cases } \\
(\%)\end{array}$ & $\begin{array}{l}\text { No. of } \\
\text { Deaths } \\
\text { (\%) }\end{array}$ & $\begin{array}{l}\text { No. of } \\
\text { Cases } \\
(\%)\end{array}$ & $\begin{array}{l}\text { No. of } \\
\text { Deaths } \\
(\%)\end{array}$ & $\begin{array}{l}\text { No. of } \\
\text { Cases } \\
(\%)\end{array}$ & $\begin{array}{l}\text { No. of } \\
\text { Deaths } \\
\text { (\%) }\end{array}$ \\
\hline$\frac{\leq 1 \text { yr }}{\text { Male }}$ & - & - & $\begin{array}{c}24 \\
(55.8)\end{array}$ & $\begin{array}{c}0 \\
(-)\end{array}$ & $\begin{array}{c}13 \\
(48.2)\end{array}$ & $\begin{array}{c}2 \\
(66.7)\end{array}$ \\
\hline Female & - & - & $\begin{array}{c}19 \\
(44.2) \\
\end{array}$ & $\begin{array}{c}0 \\
(-) \\
\end{array}$ & $\begin{array}{c}14 \\
(51.8) \\
\end{array}$ & $\begin{array}{c}1 \\
(33.3) \\
\end{array}$ \\
\hline $\begin{array}{l}\text { Total } \\
1 \text {-19yr. }{ }^{4}\end{array}$ & $\begin{array}{c}0 \\
(-)\end{array}$ & $\begin{array}{c}0 \\
(-)\end{array}$ & $\begin{array}{c}45 \\
(8.3)\end{array}$ & $\begin{array}{c}0 \\
(-)\end{array}$ & $\begin{array}{c}27 \\
(5.3)\end{array}$ & $\begin{array}{c}3 \\
(11.1)\end{array}$ \\
\hline Male & - & - & $\begin{array}{l}89 \\
(59.3)\end{array}$ & $\begin{array}{l}2 \\
(40.0)\end{array}$ & $\begin{array}{l}49 \\
(69.0)\end{array}$ & $\begin{array}{c}4 \\
(44.4)\end{array}$ \\
\hline Female & - & - & $\begin{array}{c}61 \\
(40.7) \\
\end{array}$ & $\begin{array}{l}3 \\
(60.0) \\
\end{array}$ & $\begin{array}{r}22 \\
(31.0) \\
\end{array}$ & $\begin{array}{l}5 \\
(55.6) \\
\end{array}$ \\
\hline $\begin{array}{l}\text { Total } \\
\geq 19 \mathrm{yr}^{4} \\
\end{array}$ & $\begin{array}{c}20 \\
(47.6)\end{array}$ & $\begin{array}{c}0 \\
(-)\end{array}$ & $\begin{array}{l}151 \\
(27.8)\end{array}$ & $\begin{array}{c}5 \\
(3.3)\end{array}$ & $\begin{array}{c}71 \\
(14.0)\end{array}$ & $\begin{array}{c}9 \\
(12.7)\end{array}$ \\
\hline Male & - & - & $\begin{array}{l}246 \\
(71.1)\end{array}$ & $\begin{array}{l}21 \\
(55.3)\end{array}$ & $\begin{array}{l}289 \\
(70.3)\end{array}$ & $\begin{array}{l}37 \\
(56.1)\end{array}$ \\
\hline Female & - & - & $\begin{array}{c}100 \\
(28.9) \\
\end{array}$ & $\begin{array}{c}17 \\
(44.7) \\
\end{array}$ & $\begin{array}{c}122 \\
(29.7) \\
\end{array}$ & $\begin{array}{r}29 \\
(43.9) \\
\end{array}$ \\
\hline $\begin{array}{l}\text { Total } \\
\text { TOTAL }\end{array}$ & $\begin{array}{c}22 \\
(52.4)\end{array}$ & $\begin{array}{c}0 \\
(-)\end{array}$ & $\begin{array}{c}346 \\
(63.9)\end{array}$ & $\begin{array}{c}38 \\
(11.2)\end{array}$ & $\begin{array}{c}411 \\
(80.7)\end{array}$ & $\begin{array}{c}66 \\
(16.1)\end{array}$ \\
\hline Male & - & $\begin{array}{c}0 \\
(-)\end{array}$ & $\begin{array}{c}359 \\
(66.6)\end{array}$ & $\begin{array}{c}23 \\
(53.5)\end{array}$ & $\begin{array}{c}351 \\
(69.0)\end{array}$ & $\begin{array}{c}43 \\
(55.1)\end{array}$ \\
\hline Female & 42 & $\begin{array}{c}0 \\
(-) \\
0\end{array}$ & $\begin{array}{c}80 \\
(33.4) \\
543\end{array}$ & $\begin{array}{r}20 \\
(46.5) \\
43\end{array}$ & $\begin{array}{l}158 \\
\frac{(31.0)}{509}\end{array}$ & $\begin{array}{c}35 \\
(44.9) \\
78\end{array}$ \\
\hline
\end{tabular}

${ }^{1}$ McGugan (1942); ${ }^{2}$ Davison (1942); ${ }^{3}$ Donovan and Bowman (1942a,b). ${ }^{4} \mathrm{No}$. of cases and deaths not reported according to sex for Alberta. 
Table 4. Breakdown of the human population by age and sex for the Canadian Prairie Provinces according to the 1941 Dominion of Canada census.

Age/Class Alberta Saskatchewan Manitoba

$<1$ yr.

Male

Female

$\begin{array}{rrr}7,756 & 8,761 & 6,571 \\ \underline{7,452} & \underline{8,269} & \underline{6,360} \\ 15,208 & 17,030 & 12,931\end{array}$

1-19 yr.

Male

Female

147,177

176,322

127,205

$\frac{144,425}{291,602}$

170,723

124,236

347,045

351,441

$>19 \mathrm{yr}$.

Male

271,525

292,480

244,303

Female

271,834

239.427

$\underline{221}, 069$

489,356

531,907

465,372

Total

Male

426,458

477,563

378,079

Female

369.711

418.429

$\underline{351,665}$

895,992

729,744 
b. St. Louis Encephalitis

Several excellent reviews on St. Louis Encephalitis have been published, primarily in response to a widespread epidemic of the disease throughout the central and eastern United States and southern Ontario in 1975 (Creech 1977). Important reviews include Luby 1979, Monath 1980, Kemp 1981, Tsai and Mitchel1 (1989). Mahdy et al. (1979) reviewed the response to the 1975 outbreak in southern Ontario and is of greatest relevance to Canada. This chapter is a review of the status of St. Louis Encephalitis in Canada with particular reference to findings since the 1975 outbreak.

\section{History}

St. Louis Encephalitis (SLE) was first recognized as a clinical disease in 1933, during a large epidemic in the central United States centered around St. Louis, Missouri (Chamberlain 1980). The first evidence of SLE in Canada was determined by serum neutralization tests of a 71-year-old female in Manitoba in 1941 (Donovan and Bowman 1942a,b). A further case was indicated using complement fixation in Saskatchewan in 1954 (Dillenberg et al. 1956). No further cases were identified in Canada until the major epidemic of 1975.

In 1975, 66 cases of SLE including five fatalities were reported from southern Ontario (Spence et al. 1977). Single cases were reported from Quebec (Davidson et al. 1976) and Manitoba (Sekla and Stackiw 1976). An additional four cases were detected in southern Ontario during 1976 (Weekly Bulletin, Community Health Protection Branch, O.M.H., October 8, 1976).

To the author's knowledge there have been no reported cases in Canada since 1976. In serological surveys of ca. 4,000 individuals from southern Ontario within 12 months after the 1975 epidemic, ca. $0.8 \%$ of the residents had antibodies to SLE as detected by Haemagglutination Inhibition (HAI) tests (Artsob et al. 1979). Neutralizing antibodies have been found in residents of Saskatchewan (McLintock 1976), Alberta (Hoff et al. 1970), and British Columbia (Kettyls et al. 1972).

The SLE virus was first isolated in Canada from a pool of Culex tarsalis mosquitoes collected in Saskatchewan (Burton et al. 1973). The virus has also been isolated from Culex pipiens collected in southern Ontario during 1976 (Thorsen et al. 1980) and from the brain tissue of a fatal case in southern ontario during 1975 (Spence et al. 1977).

In addition, antibodies to SLE have been detected in migratory birds in Ontario (Karstad 1965) prior to the 1975 epidemic. In southern Ontario in 1976 12.8\% of the migratory birds tested were positive for SLE but none in the subsequent two years (Dorland et al. 1979). Similarly, during limited surveillance of English sparrows in the fall of 1975 and early 1976, (29.8\%) of 57 English sparrows were seropositive for SLE (Dorland et al. 1979). Since 1977, over five summers of sentinel 
monitoring, no evidence of SLE antibody has been detected from ca. 1,000 sera per year of either English sparrows or sentinel chickens (pers. obs.). In other areas of Canada, antibodies have also been detected from moose, bull snakes and snowshoe hares in Alberta and from birds and small mammals in British Columbia (see Artsob and Spence 1979).

SLE virus is probably not endemic to Canada but enters as a northern extension of activity in the central or western United States. The cyle in western Canada would involve Culex tarsalis and that in eastern Canada Culex pipiens and Culex restuans as primary vectors.

\section{ii. Nature of Disease}

St. Louis Encephalitis is an RNA virus belonging to the family Togavuidae in the genus Flavivirus group with close antigenic relationships to Japanese Encephalitis, West Nile and Murray Valley virus (Karabatsos 1980). Serological crossreactions with such viruses as Dengue-2 and Yellow Fever virus may create false positive reactions by (HAI) or Immunoflourescence (IF) techniques. Monoclonal antibody and neutralization tests may be necessary for confirmation of antibodies (Roehrig et al. 1983). Six strains of SLE virus have been identified (Trent et al. 1980) with varying degrees of virulence (Monath et al. 1980). The strain collected from the central United States during 1975 had high virulence in mice, whereas a strain from the western United States had lower virulence (Monath et al. 1980). Actual strain determination of field isolates is therefore important in determining the potential virulence of the virus to humans.

The virus has been reported from Canada (British Columbia to Quebec) and as far south as Argentina. In addition to Canada and the United States human cases have been reported from Mexico, the Caribbean, Panama, French Guiana and Argentina (Tsai and Mitche11, in press). There are apparently two epidemiological cycles. One predominately in the western United States has been rural and involved Culex tarsalis as the primary vector. The other in the east is primarily urban with $\mathrm{Culex}$ pipiens being the major vector. With increasing urbanization in the western United States there are indications that outbreaks in western cities, (e.g. Los Angeles in 1984) may be more like the urban cycle of SLE in the east (CDC 1985).

Natural infections of SLE virus are pathogenic only to humans (Tsai and Mitchell 1986). Hamsters, mice, suckling rats and rhesus monkeys are susceptible by cranial injection (Natharson 1980). Birds are considered the most important group of hosts with species from 13 avian families identified serologically as potential hosts (McLean and Bowen 1980). Numerous species of rodents, ungulates and cricetids have also been identified as potential hosts (McLean and Bowen 1980). Several species of bats (Myotis lucifugus, Eptesicus fuscus) in the central United States have been shown to exhibit high seroactivity (Herbold et al. 1983). Virus has been isolated from hibernating bats in Texas, 
and may be a possible overwintering mechanism for the virus (Allen et a1. 1970).

\section{iii. Clinical Aspects}

SLE virus is pathogenic only to humans by natural

infection. Most humans infected are asymptomatic. Approximately 200-300 asymptomatic infections occur for each symptomatic case (Monath 1980). Of ca. 4,000 individual sera examined during the 1975 outbreak in southwestern Ontario, approximately $0.83 \%$ were positive (Artsob et al. 1979). Assuming a population base of ca. 500,000 individuals in this region, ca. 4,000 individuals were infected with SLE virus. From this infected group 66 clinical cases of SLE were identified, of which five were fatal (Joshua 1979). This fatality rate of $7.6 \%$ was similar to that of $8 \%$ found in Chicago during the 1975 outbreak (Zweighaft et al. 1979).

In Ontario, 36 female and 30 male patients were reported, with no sexual bias for the disease. All age groups were affected but cases were more common in individuals over 19 years of age $(56 / 66)$. Mortality occurred in individuals 45 or over. These cases agree with the United States data where case fatality ratios vary from 5-20\% with high ratios occurring in older patients (Monath 1980).

In Ontario, cases were placed in one of four categories:

1) mild influenza - no sequelae (4.5\%), 2) mild meningeal involvement (photophobia, insommia) with excessive fatigue and mild depression as sequelae $(36.4 \%)$, 3) increased meningo encephalitis, neck stiffness, marked photophobia, disorientation with long sequelae marked by excessive fatigue, weakness and acute depression ( $51.5 \%)$, and 4) coma and death (7.6\%). Six months after infection 31 patients had completely recovered and 34 had some sequelae (Joshua 1979). The most common symptoms were fever $89 \%$, headache $74 \%$, stiff neck $57 \%$, vomiting $56 \%$ and general malaise $48 \%$. Disorientation was observed in $45 \%$ of the cases, coma in $10 \%$ and convulsions in $3 \%$ (Joshua 1979).

of the described mortalities in Ontario during 1975 the patients (3) had coexisting disease Draisey et al. (1979). In fatal cases abnormalities are confined to the central nervous system (Suzuki and Phillips 1966). The meninges and spinal cord are infiltrated with macrophages, lymphocytes and occasionally polymorphonuclear cells. Microglial proliferation can be seen around dead and dying neurones (neuronophagia) and may be diffuse or focal in the white matter (Draisey et al. 1979).

In summary, the clinical aspects of SLE in patients from southern Ontario during 1975 were consistent with previous outbreaks and the concurrent outbreak of SLE in the United States. This reinforces the concept that SLE in eastern Canada and probably in western Canada are northern extensions of the United States epidemics.

\section{iv. Epidemiology}

St. Louis Encephalitis is primarily a disease of passeriform and columbiform birds and is transmitted by mosquitoes 
(Monath 1980). Humans are only incidentally involved with most cases occurring in August and September. In Ontario, in 1975, the first confirmed case of SLE occurred during 27 July - 3 August, and the last case on 27 September (Jones 1979). The majority of cases occurred during the last two weeks of August and the first two weeks of September. The majority of cases in the Windsor area were from within the city limited (37) with 13 cases in rural areas. This is a reflection of the population distribution of residents of the region rather than case prevalence in the city.

There are two recognized cylces of SLE transmission in northern latitudes. The rural or western cycle involves Culex tarsalis as the primary vector and is considered rural in nature. The second involves Culex pipiens as the vector and is considered urban in nature. Culex tarsalis breeds in clear to polluted water in stagnant fresh water habitats. Culex pipiens by contrast occurs primarily in polluted waters, e.g. catch basins and sewage lagoons. Adults are abundant in urban situations. Culex restuans is also prevalent in urban environments and may transmit SLE virus. The life history and population dynamics of $C$. pipiens and $C$. restuans have been detailed in Ontario (Madder et al. 1983b). Culex pipiens has been incriminated as the most probable vector of SLE in Ontario with two virus isolations (Thorsen et al. 1980) Other mosquito species of Canada which have been shown experimentally to transmit SLE virus are Aedes sticticus, Aedes vexans and Culiseta inornata (Chamberlain et al. 1959). In widespread epizootics transmission to humans may occur. SLE virus has also been isolated from other mosquito species in the United States, mites and a tick (Mitchell et al. 1980).

The overwintering mechanism of SLE virus is equivocal. SLE virus has been isolated from overwintering Culex pipiens females in Maryland and Pennsylvania (Bailey et al. 1978). Transovarial transmission of the virus occurs experimentally (Francy et al. 1981) but infection rates are low and may not be significant in nature. The virus may overwinter in birds (Chamberlain et al. 1957) or bats (Sulkin and Allen 1974). Other mechanisms may occur (Reeves 1974).

In Ontario, it would appear that the virus successfully overwintered (mechanism unknown) during the winter of 1975-76. There was no evidence of virus activity in sentinel chickens, English sparrows or mosquito pools during five years of subsequent monitoring in Ontario (1977-1983). One can assume that the virus is not endemic to the Province of Ontario. The virus potentially can be moved long distances by infected migratory birds or infected mosquitoes being dispersed from the southern United States to Canada on weather fronts.

\section{c. Eastern Equine Encephalitis (EEE)}

\section{History and Nature}

EEE has been known in North America for a long time. In 1831 a fatal neurologic disease was first described in horses along the east coast of the United States in Massachusetts. The 
virus was first isolated from horses during an outbreak in Maryland in 1931. In 1938 the virus was located from a human in Massachusetts. Subsequently the virus has been recovered during outbreaks from as far west as South Dakota, Wisconsin, Michigan and Indiana, along the eastern Atlantic and Gulf coasts in the U.S., throughout the Caribbean area and Central America and in South America. In Canada, the virus has been isolated from a horse and a migrating Junco in 1938 and 1961, respectively, in Ontario; from snowshoe hare in Alberta in 1962 and from horses in Quebec in 1972. The virus has never been isolated outside the western hemisphere.

The EEE virus is an arbovirus belonging to the family Togaviridae. There are two antigenic variants having distinct geographical distribution and characterized as North American and South American types. The latter appears to be more antigenically heterogenous than the former.

\section{ii. C1inical}

EEE clinically affects man, horses and certain species of birds. The early names of equine sleeping sickness, forage poisoning and blind staggers by which EEE was known are simply clinical manifestations of the disease in horses. In horses an initial fever of $40.5 \mathrm{C}$ develops $11 / 2$ days after infection and persists for about a day. At this stage clinical signs are anorexia and depression. A second febrile response occurs 4 to 6 days after infection with a temperature of 39.5 to $40 \mathrm{C}$ which persists for 1 to 4 days. During this second stage, neurologic signs develop including profound depression characterized by a wide stance, hanging of the head, drooping of the ears, flaccid lips and difficulty in swallowing. Foetid breath, diarrhea or constipation, and a dramatic weight loss of 50 to $100 \mathrm{~kg}$ are frequent. Some animals become restless, excitable, incoordinated, unsteady, hyperesthetic, walk in circles and into obstacles, press the head against obstacles, appear blind and deaf, grind their teeth, chew aimlessly and itch intensely with the removal of large patches of hair by chewing or scratching. Eventually the affected animals became weak, fall down and die within 5 to 10 days.

Horses that recover may have cerebral dysfunction although many appear normal.

In man, and especially in children, the disease is fulminating, with high fever, lethargy, vomiting, convulsions and the patient is gravely ill within 2 days of onset. Other signs include nuchal rigidity and coma. Mortality rates often exceed $65 \%$ among clinically ill persons. Survivors are frequently affected by neurologic and physical sequelae that include retardation, hemiparesis, aphasia, emotional instability, convulsions, hemiplegia, strabismus, impaired vision, partial deafness and speech disorders.

EEE infections produce high viremias of several days duration in birds but except for certain domesticated fowl, pigeons and pheasants, causes low mortality. 


\section{Epidemiology}

The normal transmission of EEE virus is via mosquitoes to wild rodents or wild and domestic birds. Man and horses are tangentially infected, with low to moderate viremia and although horses are probably dead-end hosts under most circumstances, the level of viremia is sufficient to infect the more efficient mosquito vectors. The female mosquito becomes infected after it takes a virus-infected blood meal. The virus replicates in the midgut cells, passes to the coelomic cavity and haemolymph and infects nearly all organs and tissues. The virus also replicates in salivary glands and probably persists there for the life of the insect. Most mosquitoes that act as biological vectors can transmit virus 10 days after initial ingestion of the infective blood meal.

In the endemic cycle, EEE virus is transmitted among birds in fresh water swamps. The principle vector appears to be Culiseta melanura, a swamp breeding mosquito that is preferentially a bird-feeding species. Epidemics of EEE in horses and man have generally originated in the vicinity of swamps, although these hosts are not infected within the swampy endemic focus. Outbreaks often occur after hot excessively rainy weather. When virus infection rates in birds are high, other mosquitoes, possibly floodwater species probably transmit the virus to horses, man and pheasants. Aedes sollicitans (a salt marsh mosquito), Psorophora confinnis (a fresh water species that breeds in temporary rain pools), Culiseta morsitans, the Culex pipiensrestuans complex, Aedes vexans and Ae. canadensis have either been associated with EEE epidemics or have been suggested as vectors.

The mechanism of EEE virus overwintering is unknown. Despite the movement of both serotypes of EEE virus in migrating birds, there is no evidence to support annual reintroduction of the virus rather than the existence of stationary foci of infection. A proposed mechanism would involve recrudescence of EEE virus from reservoir hosts. Isolations of EEE virus from cats, dogs, mice, foxes and a skunk during winter are evidence for the possibility of an overwinter cycle involving one or more of these species even though birds are more likely permanent reservoir hosts. There are also experimental infections and naturally occurring antibody in reptiles and amphibians; these species could also be overwintering hosts.

In Canada the fact that EEE virus has only been isolated occasionally from widely separated geographic areas over many years and does not appear to have become permanently established is evidence that virus is either introduced via a viremic host or infected mosquitoes are brought into an area via unusual air currents to establish a transitory focus of infection. 


\section{VECTORS}

a. Known and Potential Vectors of Western Equine Encephalitis

i. Survey of Viral Isolations

Western Equine Encephalitis (WEE) has been isolated most

frequently from Culex tarsalis, the primary vector in both Canada and the United States (Hess and Hayes 1967; McLintock and Iversen 1975; Sekla and Stackiw 1982; Sekla et al. 1980; Reeves and Hammon 1962). In Saskatchewan, 9 isolations of WEE virus were made from Cx. tarsalis in 1963 and 10 in 1965 (McLintock et al. 1970). The seasonal infection rates were $1: 145$ and $1: 169 \mathrm{Cx}$. tarsalis, respectively. In Manitoba, 33 isolations of WEE virus were made from Cx. tarsalis in 1977 and 18 in 1981; the Cx. tarsalis seasonal infection rates were $1: 347$ in 1977 and $1: 283$ in 1981 (Sekla and Stackiw 1982). WEE virus was isolated from mosquitoes in 1975, an epidemic year in Manitoba, but those mosquitoes were not identified to species (Sekla et al. 1980). Cx. tarsalis was abundant in Winnipeg that year (Brust and Ellis 1976a) and 5 out of 14 human cases were from this urban area (Waters 1976). This indirectly implicates $C x$. tarsalis as the vector in 1975 in Manitoba.

Isolations of WEE virus have been made from naturally infected Culiseta inornata, Cx. restuans, Cx. pipiens complex. Cx. peus, Mansonia (=Coquillettidia) perturbans, Anopheles earlei and several Aedes species from Canada and the United States (Hammon et al. 1945; McLintock et al. 1970; Norris 1946; Reeves and Hammon 1962; Sekla et al. 1980). In some cases the rate of infection has been higher in these secondary vectors than in $C x$. tarsalis. The rate of infection in An. earlei and Ma. perturbans in Manitoba during 1977 was 1:87 An. earlei and 1:202 Ma. perturbans respectively, compared to $1: 347 \mathrm{Cx}$. tarsalis (Sekla et al. 1980). However, these isolations were made from a waterfowl nesting area and are not representative of agricultural or urban regions of the Province. Also, populations of these species do not occur in high density in Manitoba, with the exception of $\mathrm{Ma}$. perturbans in a few marsh habitats. Ma. perturbans adult activity is only 3-4 weeks in length. Thus this species is an unlikely vector of WEE virus to equines or humans but may be involved in the amplification of the virus in the bird-mosquito-bird cycle.

In Alberta, WEE virus was isolated from Aedes vexans, Cs. inornata, and Cx. tarsalis (Shemanchuk and Morgante 1968) during a non-epidemic year. Cx. tarsalis is known to be abundant in southern Alberta (Shemanchuk 1969) and was the most likely vector during the 1963 epidemic and the 1964 epizootic of WEE in that Province.

In Manitoba, natural infections of WEE virus were found in $A e$. vexans, Cs. inornata and Ae. pionips. However, the infection rates were considerably lower than in $C x$. tarsalis (Sekla et al. 1980). Although many species can transmit WEE virus experimentally (Ferguson 1954), it is not known if their natural populations are competent vectors of the virus.

In Saskatchewan, WEE virus was isolated from Cs. inornata 11 
times during 1963-1965. The infection rate was only 1:4664 Cs. inornata in 1963, 1:1209 in 1964 and $1: 2416$ in 1965, about 1/20 the rate for $C x$. tarsalis during those years (McLintock et al. 1970). Isolations were also made from Ae. vexans, Ae. dorsalis, Ae. campestris, Ae. flavescens and Ae. spencerii. However, the number of isolations ranged from 1 to 3 per species over a 3 year period. The finding that most of the WEE virus isolations in Aedes spp. were made during an inter-epidemic year in Saskatchewan led McLintock et al. (1970) to suggest that Aedes spp. and Cs. inornata may maintain the enzootic status of the virus in that Province. Aedes spp. and Cs. inornata may also be the primary vectors of WEE virus in the Northwest Territories (N.W.T.) where 8 out of 160 reindeer, Rangifer spp., were found to have WEE antibodies (Burton and McLintock 1970). Cx. tarsalis has been collected only once from the N.W.T. (Wood et al. 1979), and is generally not found at those latitudes.

In the United States, WEE virus has been isolated from Aedes trivittatus from Iowa (Rowley et al. 1979) and Ae. melanimon from California (Hardy and Bruen 1974). Aedes melanimon is associated with the endemic WEE cycle in jack rabbits, but the role of $A e$. trivittatus in the natural transmission of WEE virus is unknown.

The status of $C x$. restuans as a vector of WEE virus in N.A. is yet to be established. In Manitoba, this species is known to be abundant because of the numerous egg rafts collected from oviposition pools (Buth 1983), but very few adults are attracted to $\mathrm{CO}_{2}$ traps or to light traps (Brust and Ellis 1976a; Gallaway 1983). Because so few females have been tested for WEE virus in Manitoba, it is not surprising that only 1 isolation has been reported (Norris 1946). It is active much earlier in the summer than Cx. tarsalis (Brust and Ellis 1976a), and may be involved in a bird-mosquito-bird cycle. Recently, Monath (1984) has suggested that $C x$. restuans may be involved in the spring amplification of St. Louis Encephalitis (SLE) virus in the Memphis, Tennessee area. Infection levels were higher in $C_{x}$. restuans than in the $C_{x}$. pipiens complex during the spring and fall of the non-epidemic year of 1979 .

The primary vectors of SLE virus in the United States are $C x . p . p i p i e n s$ in the eastern and the northern states, $C x . p$. quinquefasciatus in the southern and southeastern states (up to 39 NL), Cx. nigripalpus in Florida, and $C_{x}$. tarsalis in the western states (Mitchell et al. 1980). These are responsible for epidemic virus transmission to humans and for the enzootic virus

transmission amongst wild vertebrates. During non-epidemic years, where the ranges of $C x$. pipiens complex and $C x$. tarsalis overlap, SLE virus is isolated more frequently from the latter species

(Mitchell et al. 1980). In the eastern states, Cx. restuans and Cx. salinarius may play an important role in the sylvan enzootic transmission of SLE (Monath 1980, 1984).

SLE virus has been isolated from $C x$. tarsalis in Saskatchewan (Burton et al. 1973), and from Culex spp, mostly Cx. pipiens, from Ontario (Thorsen et al. 1979).

In the eastern and southern United States, isolation of SLE 
virus from the $C \mathrm{x}$. pipiens complex during 1974-1976 demonstrated an infection rate of $1: 178 \mathrm{Cx}$. pipiens (Monath 1979). In I11inois, Kokernot et al. (1967) reported an infection rate of 1:51. Isolations of SLE virus from $C x$. salinarius have been as high as $1: 74 C \mathrm{X}$. salinarius in the southern and eastern States, and as high as 1:73 in Memphis, Tennessee, during 1974-1976 (Monath 1979).

The list of natural and experimental vectors of SLE virus will undoubtedly continue to grow. The mosquito vectors known to date and a discussion of their significance in the SLE cycle in the United States can be found in Mitchell et al. (1980).

\section{ii. Virus Amplification}

The spring amplification phase of WEE and SLE viruses in North America begins with these viruses being present in either the mosquito vectors or wild bird reservoirs. Wild birds sustain inapparent infections with viremias sufficient for infection of mosquitoes in the well known bird-mosquito-cycle. The mechanism whereby these viruses overwinter or are re-introduced in the spring is unknown (Hess and Hayes 1967; Monath 1979, 1980).

In the spring and early summer the mosquito vectors of both WEE and SLE feed on avian hosts. CX. tarsalis and $C x$. nigripalpus significantly shift their preference from avian to mammalian hosts in mid-summer (Edman and Taylor 1968; Hayes et al. 1973; Tempelis 1975; Tempelis et al. 1965; Tempelis and Washino 1967). This shift is an important phenomenon in mosquitoes obtaining these viruses from the avian reservoir and transmitting them to humans (Edman and Taylor 1968; Reeves 1971). Passerine birds are preferred hosts of $C$. tarsalis in California and Texas, and nestlings serve as the primary amplification host (Hayes et al. 1967; Reeves and Hammon 1962). The factors that determine the rate of amplification are the numbers of infected and infective mosquito vectors (Hess and Hayes 1967; Reeves 1965; Reeves et al. 1961).

\section{Transmission}

Transmission of viruses, including WEE and SLE, has been reviewed by Chamberlain and Sudia (1961). This section deals with WEE and SLE transmission by the primary vectors and the factors that affect the successful transmission of these viruses.

Vector abundance is one of the more important factors in the successful transmission of both WEE and SLE viruses to humans (Reeves 1965). Epidemics of WEE and SLE are correlated with high levels of vector abundance (Brust 1982; Hess and Hayes 1967; McLintock et al. 1966; Mitchell et al. 1980; 01son et al. 1979; Rees et al. 1959; Reeves and Hammon 1962). However high vector populations have been present during years in which epidemic spread did not occur (Reeves et al. 1964). Vector abundance in Winnipeg, Manitoba was also greater during the non-epidemic years of 1978 and 1984 than during the epidemic year of 1981 (Raddatz 1985).

Extrinsic incubation temperature (body temperature of the 
vector) is another important factor in the transmission of WEE and SLE viruses to vertebrates (Hess et al. 1963; Reeves 1965; Reeves et al. 1964). After acquiring an infective blood-meal, Cx. tarsalis incubates WEE or SLE virus for several days before being able to biologically transmit the virus to a new host. The temperature of extrinsic incubation in the vector may be the reason WEE outbreaks have occurred at or above the $21 \mathrm{C}$ June isotherm whereas SLE outbreaks have occurred at or below the $21 \mathrm{C}$ isotherm (Hess et al. 1963).

WEE and SLE virus transmission varies with vector efficiency (competence) of the particular strain of vector (Reeves 1965). Vector competence and its role in virus transmission has been an active topic in recent years, and a number of reviews are available (Gubler et al. 1982; Hardy et al. 1983; McLintock 1978; Mitchell 1983a). These should be consulted for details on Salivary Gland Infection (SGI) Barrier, Mesenteronal Infection (MI) Barrier, Mesenteronal Escape (ME) Barrier and leaky midgut. A recent study has shown that the MI barrier to WEE virus in $C X$. p. pipiens may be due to the failure of the virus to penetrate the gut epithelium (Houk et al.1986).

The virus dose acquired by a vector plays a role in the successful transmission to another host. In a Manitoba strain of Cx. tarsalis, infected with a WEE virus isolate from Manitoba, the infection threshold in $50 \%$ of $C x$. tarsalis, infected via a bloodmeal from a viremic chick, was $\log 2.3$ Tissue Culture Infective ( $50 \%$ leve1) Dose (TCID50) per $0.3 \mathrm{ml}$ of donor blood. Transmission rates were near $100 \%$ after 25 days (Henderson et a1. 1979). Biological transmission of WEE virus by $C x$. tarsalis, has been shown to be a minimum of 4 days at $24 \mathrm{C}$ when transmitted from chick to chick or chick to mouse (Henderson et al. 1979; Hayles et al. 1972; Hayles 1976). From chick to mouse, Henderson et al.(1979) obtained $76 \%$ transmission. From chick to chick $81 \%$ transmission occurred over 31 days. Hayles et al. (1972) obtained $86 \%$ transmission from chick to chick over 44 days.

Biological transmission of SLE virus in $100 \%$ of infected $C x$. p. pipiens may be reached after 12 days of extrinsic incubation, whereas transmission rates of only 18 to $29 \%$ occurred in $C x$. $p$. quinquefasciatus after a comparable incubation period.

Transmission rates in the latter subspecies reached $100 \%$ after 19 to 26 days of extrinsic incubation (Chamberlain et al. 1959).

Strains of $C x$. tarsalis which are highly resistant to WEE virus infection have been isolated after prolonged selection. Resistance has been associated with a mesenteronal barrier, because both refractory and parental strains were equally susceptible to infection by intrathoracic injection. Inheritance of WEE virus susceptibility is probably polyfactorial in $C x$. tarsalis (Hardy et al. 1978). Genetic selection in laboratory populations and seasonal variations amongst field populations of Cx. tarsalis have also been shown to affect the infection and transmission rates of WEE virus (Hardy et al. 1979).

Transovarial transmission of SLE virus by $C_{x}$. pipiens complex mosquitoes (Francy et al. 1981; Hardy et al. 1984) and by 
Cx. tarsalis, Ae. epactius, and Ae. atropalpus (Hardy et al. 1984) has been demonstrated experimentally. Francy et al. (1981) obtained low transmission rates in progeny from the first ovarian cycle, but the transmission rates increased as much as 30 fold in the second cycle. Hardy et al. (1984) obtained much higher rates of infection in progeny from the first ovarian cycle in the species they tested, including laboratory populations of $C x$. pipiens and $C x$. tarsalis. In both studies low temperature maintenance $\left(18^{\circ} \mathrm{C}\right)$ of larvae and adults was necessary to obtain successful transmission to $\mathrm{F} 1$ larval or adult progeny (Francy et al. 1981 and Hardy et al. 1984).

iv. Overwintering of Vectors

Females of $C x$. tarsalis and $C x$. p. pipiens overwinter as inseminated, nulliparous females (Bellamy and Reeves 1963;

Bennington et al. 1958b; Blackmore and Dow 1962; Spielman and Wong 1973a). Low temperature and short photoperiod induce a facultative ovarian diapause. Females seek nectar rather than a blood-meal to build fat body reserves for overwintering (Eldridge 1968; Eldridge and Bailey 1979; Harwood and Halfhill 1964; Mitchell 1981,1983a; Spielman 1971a; Wilton and Smith 1985). The life stages sensitive to these induction cues are fourth instar larvae and pupae (Eldridge 1968; Reisen 1986, Reisen et al. 1986b; Spielman and Wong $1973 \mathrm{a}, \mathrm{b})$. In southern Jntario initiation of diapause begins in late July, with $90 \%$ of emerging adults being in diapause in mid-August (Madder et al. 1983a). If vector control was necessary to halt an epidemic in progress, larviciding to control $C x . p$. pipiens in Ontario would not affect transmission after mid-August. Over $90 \%$ of the newly emerged females would not be involved in blood-feeding and therefore would be unable to transmit a pathogen.

Diapausing females of $C x . p$. pipiens and $C x$. tarsalis can be induced to take a blood-meal if they are warmed to $25 \mathrm{C}$ for 1 to 3 days and placed in close association with a suitable host (Eldridge and Bailey 1979; Mitchell 1981, 1983b). However, diapause is not terminated under these conditions. The females are induced to blood-feed by avoiding the host-seeking step in the biting cycle. If diapausing females are placed in a $3.8 \mathrm{~L}$ container for blood-feeding instead of a $0.24 \mathrm{~L}$ one, adding hostseeking to the feeding cycle, the females do not feed (Mitchell 1981,1983b). Some diapausing females of $C X . p$. pipiens and $C X$. tarsalis that blood-feed, when placed in close association with the host, fail to use the blood for egg development. This occurred when females were kept at $15 \mathrm{C}$ and a short photophase for 1 week (Arntfield et al. 1982; Eldridge and Bailey 1979; Mitchell $1981,1983 \mathrm{~b})$. However, this probably has little significance to what occurs in nature because diapausing females would not be in the host-seeking mode and would not take a blood-meal.

Fat body size in $C x$. tarsalis increases after females take a carbohydrate meal. In nature, females have significant lipid reserves in the fall and winter months (Bennington et al. 1958b; Schaefer and Washino 1969, 1970; Takata and Harwood 1964). 
Females of $C x$. p. pipiens also develop considerable fat reserves on a carbohydrate diet when maintained at 5 C (Tekle 1960) but less than prehibernating Cx. p. pipiens in nature (Eldridge 1968). When females of $C x$. p. pipiens are kept at a short photoperiod at $10 \mathrm{C}$, considerably more fat reserves are accumulated than in $C x$. p. quinquefasciatus (Eldridge 1968).

Females of $C x$. tarsalis in nature become increasingly cold tolerant as fall progresses to winter (Anderson and Harwood 1966). Adults collected in October in Washington State survived longer at low temperatures than did adults collected in August. Females have been shown to accumulate a larger percentage of unsaturated fatty acids in their fat body in response to low temperatures and short photoperiods. This accumulation may provide the increased cold tolerance (Harwood and Tanaka 1965).

Overwintering sites for $C x$. tarsalis in Canada are largely unknown. Shemanchuk (1965) found that overwintering adult females survived in badger, skunk and marmot burrows in southern Alberta. Hudson (1978) found several species of mosquitoes overwintering in rock piles, root cellars and badger burrows in central Alberta. However no overwintering females of $C x$. tarsalis were found during the 3 winters (November to March) in which numerous adults of An. earlei (445) and $C x$. territans (106) were found.

In the western United States, overwintering females of $C x$. tarsalis have been found in abandoned mines (Blackmore and Winn 1956; Chapman 1961; Mitchel1 1979), outdoor food storage cellars (Keener 1952), under loose rock on canyon and cliff slopes

(Harwood 1962; Rush 1962), under bridges (Dow et al. 1976), inside culverts (Kliewer et al. 1969) and in rodent burrows (Bennington et al. 1958b).

The spring emergence of $C x$. tarsalis from natural overwintering sites is correlated with the time of the spring inversion of soil temperatures (Bennington et al. 1958a; Dow et a1. 1976; Shemanchuk 1965). In southern Alberta Cx. tarsalis emerged from animal burrows in April and May (Shemanchuk 1965).

\section{b. Known and Potential Vectors of Eastern Encephalitis Virus}

There have been numerous isolations of EEE virus and antibodies in a variety of birds and mammals in Canada (Artsob and Spence 1979), yet no infected mosquitoes have been identified to date in this country. In the United States, Culiseta melanura appears to be the primary enzootic vector (Chamberlain 1987). Female Cs. melanura are predominately ornithophilic (Hayes 1961; LeDuc et al. 1972) and circulate EEE virus among avian hosts in swamplands and margins in endemic areas (Grimstad 1983; Howard et a1. 1983). Culiseta melanura overwinters in the larval stage and is restricted in its distribution in Canada to extreme eastern Ontario and southern Quebec (Chant et al. 1973; E11is and Wood 1974; Wood et al. 1979). It is, therefore, unlikely that Cs. melanura constitutes a primary role in EEE virus transmission in Canada, unless there are isolated pockets of infection among resident birds which are so far undetected.

Eastern equine encephalitis virus has been isolated from 
many additional genera and species of mosquitoes, some of which are probably responsible for virus transmission to mammals, including horses and man. Mansonia perturbans, a widely distributed and viscious biter in Canada, was first identified as a vector for EEE virus by Howitt et al. (1949). Since then, it has been suggested repeatedly as an important vector in the spread of EEE virus out of marshland areas and to mammal hosts (Srihongse et al. 1980; Grimstad 1983). Aedes sollicitans and Ae. taeniorhynchus have been implicated as important vectors in the eastern saltmarshes of the United States (Crans 1977). Because of the restricted distribution of the former (Wood et al. 1979), and absence of the latter in Canada, they are undoubtedly of no consequence here. Aedes vexans, Ae. canadensis, and Ae. cantator, all of which occur more widely in Canada (Wood et al. 1979), have been suggested as possible vectors of EEE virus (Grimstad 1983). Infected Culex restuans, $C x$. pipiens and $C s$. morsitans have been recovered in New York (Srihongse et al. 1980), but their role in the epidemiology of EEE is unknown.

In Canada, therefore the primary vector candidates for EEE virus are Mansonia perturbans and perhaps Aedes spp. Although Culiseta melanura is present in eastern Canada, it is rare and limited in distribution. It is possible, however, that low levels of virus may be circulated among bird populations where $C s$.

melanura does occur, and an alternate vector is then responsible for transmission to mammal hosts. Vectors responsible for exposed animals in Alberta and Saskatchewan (Artsob and Spence 1979) are a matter of conjecture, but Ma. perturbans would be an interesting species to examine in more detail.

\section{c. Known and Potential Vectors of California-group Viruses} California encephalitis virus

California encephalitis virus is reported to cause human illness (Hammon and Reeves 1952), but only three cases have been identified in North America (California) (Monath 1979). This virus is widespread in the United States, having been reported from California, Utah, New Mexico and Texas (Calisher 1983). This virus has only recently been isolated from Canada, from a pool of Culiseta inornata collected from Selkirk/Oak Hammock, Manitoba (Artsob et al. 1985). California encephalitis virus is generally believed to be transmitted by Aedes spp. (Le Duc 1979), including Ae. dorsalis, Ae. melanimon, Ae. nigromaculis and Ae. vexans (Turell and LeDuc 1983). However, isolations have also been made from Culex tarsalis, Anopheles freeborni, and Psorophora signipennis, as well as Cs. inornata in the United States. It is interesting that in laboratory trials conducted by Reeves and Hammon (1952), Cx. tarsalis and Cs. inornata were unable to transmit California encephalitis virus after feeding on an infected blood source.

All species of mosquitoes from which California encephalitis virus has been isolated in the field, occur in Canada (Wood et al. 1979). Some of these, such as Ae. vexans, Ae. dorsalis, Cs. inornata and Cs. tarsalis are widespread, often abundant, and 
known to bite man. However, the prevalence of California encephalitis virus is very low, and considering the rarity of human cases, does not deserve primary concern in Canada at the present time.

Snowshoe Hare virus

Snowshoe Hare virus has been implicated in the cause of human disease (Feltz et al. 1972; Lindsey et al. 1976), and has been isolated from all provinces and territories in Canada (Artsob 1983). Most isolations from mosquitoes have been recovered from Aedes spp., especially the univoltine species predominant in the boreal, subarctic and arctic regions where Snowshoe Hare virus is endemic (LeDuc 1979). A summary of naturally infected vector species was provided by LeDuc (1979), and includes Aedes canadensis, Ae. cataphylla, Ae. cinereus, Ae. communis, Ae. excrucians, Ae. fitchii, Ae. hexodontus, Ae. implicatus, Ae. intrudens, Ae. punctor and Ae. vexans. Only the latter, Ae. vexans, is multivoltine, and of these has been shown to become infected and to transmit the virus in the laboratory (LeDuc 1979). Turell and LeDuc (1983) included Ae. stimulans, Ae. triseriatus, and Ae. trivittatus among additional sources for virus isolations. Culiseta inornata may also be an important vector in more southern latitudes. Morgante and Shemanchuk (1967) discovered Snowshoe Hare virus in adult $C s$. inornata collected from mammal burrows in southern Alberta. Mclean et al. 1974 demonstrated the ability of Cs. inornata to acquire and transmit the virus in the $l \mathrm{ab}$, and for the virus to persist for prolonged periods of time within these mosquitoes at low temperature (McLean et al. 1975, a,b). Snowshoe Hare virus has also been isolated from the black fly, Simulium malyschevi in Alaska (Ritter and Feltz 1974), but its role as a vector for the virus is unknown.

Aedes species are generally believed to be the primary vectors of Snowshoe Hare virus over most of its range. In some areas, however, Cs. inornata clearly may play an important role in maintenance and transmission of the virus. More research is needed to illucidate the relative importance of the various vector species present in endemic areas.

\section{Jamestown Canyon virus}

Jamestown Canyon has been implicated in human disease in Canada from a single isolation in Ontario (Deibel et al. 1983). Virus isolations over most of the known range from Aedes spp., including Aedes cantator, Ae. communis, Ae. melanimon, Ae. punctor, Ae. sollicitans, Ae. stimulans, Ae. thelcter, Ae. trivittatus, and Ae. vexans, but Anopheles crucians, An. punctipennis, An. quadrimaculatus, Psorophora columbiae, and Ps. discolor have also provided sources of isolations (Turell and LeDuc 1983). However, in the western United States, Culiseta inornata has been the major source of isolation in the field, and is known to transmit the virus in the laboratory (Turell and LeDuc 1983). Isolations of Jamestwon Canyon virus are also known for the tabanids, Chrysops cincticornis, Hybomitra lasiophthalma and 
H. nuda (Turell and LeDuc 1983), though their role in virus dissemination is unknown.

Virus isolations and positive antibody determinations for Jamestown Canyon virus are widespread in Canada (Hoff et al. 1969; Iversen et al. 1973; MacFarlane et al. 1981, 1982; Artsob 1983). Since many of the mosquitoes from which this virus has been isolated occur and may be widespread in Canada, the potential for additional human clinical cases is clearly present.

\section{Trivittatus virus}

Trivittatus virus is suspected as causing human disease (LeDuc 1979), but there is no available evidence to support this in Canada. There is, in fact, only one isolation of this virus from mosquitoes (Aedes trivittatus) from extreme southwestern Ontario (Thorsen et al. 1980). There have been no other virus isolations or seropositive vertebrates reported for Canada (Artsob 1983). The low prevalence of Trivittatus virus might be expected, considering that Aedes trivittatus, which is probably the principal vector for the virus in the United States, is quite restricted in Canada (Wood et al. 1979). Nonetheless, Trivittatus virus has been isolated from many other mosquito species in the U.S., including Aedes atlanticus/tormentor, Ae. dorsalis, Ae. infirmatus, Ae. sollicitans, Ae. taeniorhynchus, Ae. triseriatus, Ae. vexans, Anopheles crucians, Mansonia perturbans, Culex nigripalpus, Cx. pipiens, Culiseta inornata, and Psorophora signipennis (Turell and LeDuc 1983).

Many of the California group viruses have been isolated in the field from a large number of blood-feeding arthropods. For example, Snowshoe Hare, Jamestown Canyon, and Trivittatus viruses have been recovered from 20, 18, and 14 species respectively. However, in all cases, the number of vectors that are in fact important in the spread of the virus from one vertebrate host to another is limited. Because of the blood-feeding habit, it is conceivably quite easy for an arthropod to acquire virus from a suitable viremic host. Other factors, including the morphological, physiological and behavioural characteristics of the arthropod, however, influence the competency of the arthropod to transmit the virus upon subsequent blood meals. It takes a considerable amount of time and research effort before these levels of competency can be established, and it is in this area that our knowledge of the status of the California group viruses is greatly deficient in Canada.

\section{d. Possible Overwintering of Viruses}

In Canada, endemic foci of mosquito-borne viruses can persist only where suitable mechanisms for winter survival are available and highly successful. Unfortunately, the amount of information on overwintering of these viruses is sadly lacking.

There are essentially three possible mechanisms by which viruses may be maintained in invertebrate vector populations, as described by Reeves (1974). Viruses may survive within the primary mosquito vector populations where long-lived adults obtain 
a viremic blood-meal before entering hibernation, and then retain the infection through hibernation, to transmit the virus with the first blood-meal(s) in the spring. On the other hand, primary mosquito hosts may transmit the virus transovarially, and maintain infected vectors in the population even in the absence of suitable, viremic vertebrate hosts. It is also possible that an endemic cycle exists involving arthropods other than the known primary mosquito vectors. In this case, these other arthropods may overwinter the virus, by either or both of the above mechanisms described for overwintering in the mosquito hosts.

Clearly, there is an urgent need to establish the mechanisms responsible for successful overwintering of mosquito-borne viruses in Canada. We must be able to identify endemic foci, if present, and determine the contribution of both primary and alternate arthropod vectors. Without this knowledge, reasonable predictions on factors affecting disease outbreaks cannot be expected.

\section{California Group Viruses}

Four California group viruses are known from Canada:

Snowshoe Hare virus, Jamestown Canyon virus, Trivittatus virus, and California Encephalitis virus.

California Encephalitis (CE) has been isolated only from Manitoba in Canada (Artsob et al 1985), and though known to cause disease in humans, is of little importance in this county at the present time. Transmission probably involves primarily Aedes spp., but CE virus has been isolated from other general (Turell and LeDuc 1983). Transovarial transmission may be a possible overwintering mechanism among vectors of $C E$ virus. Smart et al. (1972) and Crane et al. (1977) have provided evidence that CE virus was transovarially transmitted by Ae. dorsalis in Utah. However, Reeves (1977, in LeDuc 1979) has not been able to find CE virus in overwintering Ae. melanimon in California. More research is needed before any conclusion can be reached.

Trivittatus virus is also present in Canada (Artsob 1983) and is of little importance at the present time. Aedes trivittatus is probably the primary mosquito host (LeDuc 1979). Overwintering via transovarial transmission was first suggested by Pinger et al. (1975) and Watts et al. (1976), and later confirmed in the field by Andrews et al. (1977).

Jamestown Canyon (JC) virus is apparently associated with white-tailed deer (LeDuc 1979), and not known to cause disease in man. Very little information is available on JC virus maintenance in the field, but Berry et al. (1977) did isolate the virus from larval Ae. triseriatus in Ohio. This is the only known record for overwintering of JC virus. Obviously, a great deal remains to be done to develop a meaningful understanding of overwintering for this virus.

Snowshoe Hare virus is one member within the California group viruses for which there is good evidence that mosquito vectors are important sources for winter maintenance. This virus has repeatedly been isolated from larvae of various univoltine Aedes spp. in the North, including Ae. communis, Ae. hexodontus, 
Ae. canadensis and Ae. implicatus (McLean et a1. 1975, 1977;

McLintock et al. 1976). Despite these isolations, however, definitive studies on subsequent transmission by adults of these various Aedes spp. have not yet been done. Further south, Culiseta inornata may be an important overwintering source for Snowshoe Hare virus. Culiseta inornata has the ability to transmit the virus (Mclean et al. 1974) and to survive and remain infected for extensive periods at low temperatures (McLean et al. 1975). Again, however, Snowshoe Hare virus has not been isolated yet from overwintering, blood-fed adult $C s$. inornata, and the exact role of $C s$. inornata for maintaining the virus is not known (LeDuc 1979).

Although there is good evidence for transovarial transmission and overwintering for some California group viruses (e.g., LaCrosse and Keystone, see Watts and Eldridge 1975, Tesh 1984), found in Canada, the evidence is still largely circumstantial. Hypotheses developed so far are based on infected larvae recovered from the field, early appearance of seroconversion in sentinel hosts, or transovarial transmission in the laboratory. We are still a long way from determining the importance of mosquito vectors in overwintering virus maintenance in terms of epidemiology of the various California group viruses found in Canada.

St. Louis Encephalitis Virus

There are no Canadian studies in which overwintering SLE virus in arthropod vectors has been investigated. Inference must be made from research conducted in the United States.

All known primary mosquito vectors either survive the winter as hibernating adults (Culex pipiens pipiens, Cx. tarsalis) or as actively reproducing populations ( $C x$. pipiens quinquefaciatus, $C x$. salinarius). Only $C x$. pipiens and $C x$. tarsalis occur in Canada and may be involved in SLE virus transmission. Most overwintering females do not initiate host seeking and blood feeding prior to hibernation (e.g., Bellamy and Reeves 1963; Wilton and Smith 1985). SLE virus has been isolated from overwintering mosquitoes (Bailey et al. 1978), but their ability to transmit the virus in spring remains to be shown. Although a possible component to SLE epidemiology, overwintering of the virus in previously blood-fed hibernating female mosquitoes is perhaps of minor importance at best.

The ability of other arthropods to transmit and overwinter SLE virus is poorly known. Although Aedes spp. transovarially transmit California group viruses (bunyaviruses) and have been reported as carrying SLE virus, they have not been implicated in overwintering SLE virus in the field. SLE virus has been isolated from mites (Dermanyssus spp., Ornithonyssis sp.) and ticks (Dermacentor variabilis, Argas arboreus) (see Mitchell et al. 1980), but their role in the epidemiology of SLE is unknown.

Although not yet demonstrated in field populations, transovarial transmission of SLE has been demonstrated experimentally under laboratory conditions. Two Aedes spp. 
(epactius and albopictus), Cx. pipiens and $C \mathbf{x}$. tarsalis successfully transmitted virus to their offspring (Francy et al. 1981, Hardy et al. 1981, Tesh 1984), the efficiency of which may be temperature related (Hardy et al. 1981). Hardy et al. (1981) and Nayar et al. (1986), have also provided additional support for vertical transmission of SLE virus in both Culex and Aedes spp.

\section{Western Equine Encephalitis}

There is no evidence to support transovarial transmission of Western Equine Encephalitis virus to provide the overwintering population of Culex tarsalis with a source of virus. In Canada, as in most of its range, adult nulliparous females overwinter in a condition of ovarian diapause (Bellamy and Reeves 1963; Spielman and Wong 1973). Thus, without evidence for transovarial transmission, it is unlikely that the principal vector for WEE virus can contribute to maintenance of the virus over winter in Canada. There is only one report of WEE isolation for 'over wintering' Cx. tarsalis. Blackmore and Winn (1956) collected WEE virus from a single pool of $C x$. tarsalis during December in Colorado. However, it was not shown whether infected mosquitoes could survive until spring.

Culex tarsalis can retain WEE virus and subsequently transmit the pathogen to a vertebrate host after at least 109 days at reduced temperatures (Bellamy et al. 1958). It is, therefore, possible that under climatically favourable conditions in part of its range, dormant $C x$. tarsalis may maintain the virus.

The role of alternate invertebrate vectors as a possible mechanism for overwintering WEE virus has been investigated, but as yet no positive results are available. Reeves et al. (1947) isolated WEE virus from the bird mite Liponyssus sylvarium in California, but the role as an overwintering source has not been substantiated. The swallow bug, Oeciacus vicarius, though capable of overwintering a serologically similar virus, Fort Morgan, is incapable of transmitting WEE virus (Calisher et al. 1980). WEE virus has been isolated during the summer from numerous presumably secondary mosquito vectors, including Aedes, Anopheles, Culiseta, and Mansonia species. There has been insufficient research to conclusively eliminate these vectors as a possible means of overwintering WEE virus, though a high probability of success is unlikely from what we know at the present time.

\section{Eastern Equine Encephalitis (EEE)}

Although there have been numerous attempts to isolate EEE virus from overwintering vector mosquitoes or to demonstrate vertical transmission of the virus, there have been very few successes (Rosen 1987). Only once (Hayles et al. 1960) has EEE virus been isolated from $C s$. melanura larvae in the field, as possible evidence for transovaxial transmission. EEE virus has been isolated from mosquitoes (Chamberlain and Sudia 1961), but as Rosen (1987) points out, this could have been the result of contamination from infected females. Watts et al. (1987) failed to detect transovarial transmission in either Cs. melanura, Ae. 
cantator or Mansonia perturbans. Considering the overwhelming evidence against EEE virus overwintering in mosquito vectors in the United States, and considering that EEE virus has not yet been isolated from field-collected mosquitoes in Canada, it is probably safe to conclude that this mechanism for overwintering in a mosquito vector is of no consequence in the epidemiology of $\mathrm{EEE}$ in Canada at the present time.

\section{Summary}

The evidence for mosquito vectors serving as an overwintering mechanism for arboviruses was summarized by Rosen (1987). For the California group viruses found in Canada (Snowshoe Hare, Jamestown Canyon, Trivittatus, and California), there is ample evidence to support transovarial transmission as a means for maintenance of the viruses, though other mechanisms may be involved as well. For St. Louis encephalitis virus (a Flavivirus), the evidence is weak, and considerably more research is required before any conclusions can be made. For the two Alphaviruses found in Canada (WEE and EEE), there is no evidence that mosquito vectors contribute to maintenance through the winter. 


\section{SURVEILIANCE}

a. Larval and Adult Surveys

i. Larval Surveys

Larval surveys are used to identify the sources of problem mosquitoes, and to determine their relative abundance. This information is essential to the operation of an efficient control program.

\section{Equipment}

A white enamel or plastic dipper, about $10 \mathrm{~cm}$ in diameter, is most often used for collecting mosquito larvae. Small larvae are most easily seen against the white background. Usually a long wooden handle is attached to allow easier sampling.

A large squeeze bulb syringe of the type used for food basting is useful for sampling breeding sites unsuited for dipping, such as tree holes or rock crevices. For deep holes a length of tubing can be added to the syringe. A flashlight can also be used to improve visibility under these conditions.

During extensive field sampling, a supply of small, screwcap vials containing $70 \%$ alcohol should be on hand. Larvae can be transferred from dippers to these vials with eye-dropper pipettes. A note book should be carried to record field data. A strainer is useful to transfer larvae to clean water when sampling darkcolored or particle-laden water. Rubber wading boots are usually essential to sample an area thoroughly.

\section{Procedures}

In water bodies on the ground, mosquito larvae are usually found around the margins, associated with emergent vegetation and debris. When dipping is planned, the water body must be approached slowly and carefully. If the water is disturbed or a shadow cast, mosquito larvae often dive to the bottom and can not be collected in a dip sample. Because larvae tend to "bunch up" rather than being uniformly distributed, it is often necessary to sample several parts of a water body. Each site should be sampled frequently during the breeding season.

Collected larvae can be preserved in alcohol-filled labelled vials soon after collection for later identification. If possible, larvae should be heat-killed by placing them in hot water. Some or all larvae can be kept alive and raised through to adults as an aid to identification.

Accurate field notes are important. Each water body should be given a code designation and described, and a notation should be made of the date, number of dips made, number of positive dips, and an approximation of the number of larvae collected. The species found should be added upon identification.

\section{Adult Surveys}

Adult mosquito surveys provide information on the relative abundance and seasonal and spatial distribution of species in an area. This can be important in providing forewarning of a disease 
potential by producing data on known vector species. Adult surveys can also serve to evaluate on-going control programs.

\section{Light Traps}

Traps utilizing light as an attractant are widely used as a method of surveying adult mosquitoes. The most commonly used trap is the New Jersey trap, which has a standard household light bulb and requires a 110 volt source of electric power. An automatic timer or photo-cell can be used to control the trap so it operates only during hours of darkness. This is a durable, low maintenance trap that attracts large numbers of mosquitoes.

The New Jersey traps' requirement for a 110 volt power source restricts its use. For remote areas or other situations where electricity is not available, a trap known as the CDC miniature light trap can be used. This is a battery powered trap with a small flashlight-type bulb as an attractant. Usually the batteries will provide only one night trapping and therefore the CDC trap, while portable, requires considerable maintenance. This trap usually catches fewer mosquitoes than the New Jersey trap.

The CDC trap is often used to collect living specimens. New Jersey traps usually kill the entrapped mosquitoes, although the killing jar can be replaced with a mesh bag for live-trapping. Carbon dioxide from dry ice may be used in conjunction with light to enhance a trap's effectiveness.

Proper location of light traps is important. Traps should be installed so that the light source is 165 to $180 \mathrm{~cm}$ above the ground. Traps should be at least 9 meters from buildings, out of the wind as much as possible, and away from competing light sources.

A number of factors must be considered when interpreting light trap catch data. There are considerable differences among mosquito species in their response to light. Depending on the species in the sample area, it may be necessary to use other adult sampling methods in conjunction with light traps to get meaningful data on the relative abundance of mosquitoes. Fluctuations in traps catch numbers do not necessarily reflect changes in the number of mosquitoes in the area. Light trap catches generally decrease during full moon periods. Also, trap catches are affected by changes in flight activity which are governed in part by environmental factors such as temperature and wind.

The degree of accuracy of light trap surveys increases with the number of traps used, which depends mainly on the number of traps and the manpower available.

\section{Biting Collections and Landing Counts}

Collecting and counting mosquitoes as they bite or land to bite is an effective way of determining the relative abundance of species that feed on man and are which therefore potential vectors. Biting collections are made by sucking mosquitoes into an aspirator tube as they begin to bite, usually on the bare legs from knees to ankles. They are then blown into a killing or holding tube for subsequent identification. If mosquito numbers 
are very high, biting collections may become impractical and landing counts are conducted. A landing index (no. of mosquitoes landing per unit time) is made by counting, and collecting if desired, mosquitoes landing on a certain area of the collector, such as the front of the pants, over a measured period of time.

For reliable spatial and temporal comparisons, biting collections and landing counts should be standardized as much as possible. Sampling should be done at approximately the same relative time from day to day, and similar conditions regarding vegetation and wind exposure should be selected. Because of differences in attractiveness among individuals, it is desirable that the same people be used throughout a survey. Similarly colored clothes should be worn by all participants from day to day.

The flexibility of biting and landing counts makes this a very useful method of surveying adult mosquitoes. Counts can be made over a wide area with little preparation, and can be done during daylight hours or, with the aid of a flashlight, at night.

\section{Bait Traps}

Traps using live animals or carbon dioxide (dry ice) have been developed for mosquito surveys. Such traps attract only biting insects and can be used in areas without electric power. However, they require daily maintenance as they are usually baited at dusk and the insects and bait are removed at dawn. Live animals also deserve special care, and procedures must be in compliance with all current Animal Care guidelines.

Bait traps may be small, using a chicken, guinea pig, or dry ice as an attractant, or large enough to be baited with livestock such as calves. All such traps are equipped with a one-way entrance such as an inwardly-directed funnel or V-shaped slot. Where larger animals are used, traps with portable sides for installation after a given exposure period may be helpful.

\section{Resting Stations}

Many species of mosquitoes, especially Culex and Anopheles, are inactive during the day and may be found resting in dark, cool, humid sheltered places. Careful counting of mosquitoes in these daytime shelters can give a comparative index of population densities. Sites that should be examined include barns, culverts, bridges, caves, and animal burrows. A portable light source is essential for many of these areas. An experienced observer often can visually determine the sex and species present, as well as make counts without collecting the mosquitoes. Usually some specimens are taken for laboratory identification. If live specimens are required, such as for virus isolation, an aspirator and holding container are necessary. It is important that counts and collections be made at the same time of day and in the same manner for accurate comparisons of results.

\section{Population Dynamics, Activity}


Information on natural mortality and reproductive potential in $C x$. tarsalis and $C x$. p. pipiens is generally lacking. Most of the published information on the population dynamics of these species is based on laboratory colonies. A thorough review of their bionomics can be found in Mitchell et al. (1980).

At 20 to $21 \mathrm{C}$, the development time for the larvae of $C x$. tarsalis is 8 to 14 days, with a mean of 11 to 12 days. Allowing 3 to 4 days for pupal development, the time from egg to adult is approximately 15 days (Brennan and Harwood 1953; Hagstrum and Workman 1971).

The average development time for larvae of the $C_{x}$. pipiens complex at 26 to $27 \mathrm{C}$ is 6 days (Petersen and Willis 1972; Shelton 1973). Allowing 3 days for pupal development, the time from egg to adult is about 9 days.

Estimates of adult survival rates in natural populations of Cx. tarsalis have been made by Nelson et al. (1978) and Reisen et al. (1983). From mark-release-recapture studies, Nelson et al. (1978) estimated daily survival to be in the range of 64 to $77 \%$. Reisen et al. (1983) estimated daily survival rates of resting females during May to August to be 74 to $87 \%$.

A statistical model of the population dynamics of $C x$. tarsalis, based on biological studies, has been developed to quantify population increases in this species (Moon 1976). The parameters of the population dynamics were based on the Bakersfield, California area. The reproductive season in that region is about 9 months annually (Bellamy and Reeves 1963; Reisen et al. 1986a). In Washington state, the active reproductive period is around 6 months (Anderson and Harwood 1966), while in Manitoba it is approximately 4 months (Brust 1982).

Moon (1976) based his expected population increases on two week intervals, beginning 20 March. Calculating 2+ gonotrophic cycles by 17 April, an expected population increase from 1046 to 39,537 was predicted over 4 months. This increase was based on an adult daily survival rate of $85 \%$.

An increase in trap catches of $C x$. tarsalis of this magnitude occurs over a much shorter period of time in regions where this species has been studied. In Manitoba, a 10 to 30 fold increase in $C x$. tarsalis females trapped occurred within a 2 to 3 week period (Brust 1982). However it is not known whether the size of trap collections reflect population levels or primarily flight activity levels.

Raddatz (1985) developed a biometeorological model that related Winnipeg's mean daily count of $C x$. tarsalis (total daily counts averaged over one week periods) to the antecedent ( 3 week lag) period of temperature and precipitation. The effect of the weather over the trapping period was partially accounted for by the multiplicative activity-level factor which was an exponential function of mean daily temperatures (mean daily maximum and minimum temperatures averaged over one week periods). This factor had a value of 1.00 for a mean daily temperature of $15 \mathrm{C}$, dropping to 0.22 for $0 \mathrm{C}$ and rising to 2.72 at $25 \mathrm{C}$. In this way the model was able to predict trap collections of $C_{X}$. tarsalis, and 
presumably population size, 3 weeks in advance of the collections.

The number of generations of $C x$. tarsalis in Manitoba is

also affected by environmental conditions, mainly seasonal

temperatures and the timing and amount of precipitation.

Undoubtedly the timing of adult emergence from winter

hibernation sites is important in the dynamics of this species. over a period of 10 years (1975 to 1984) the dates of the first Cx. tarsalis male(s) trapped in Winnipeg in spring ranged from 11 May to 26 June, with a median date of 6 June (Ellis 1984). The first $C x$. tarsalis egg rafts collected over a 5 year period in Winnipeg, Manitoba (1980 to 1984) ranged between 7 May and 14 June (Brust unpublished). During 1980 to 1984, egg rafts were found up to a month before females were captured in light traps in the Winnipeg region, possibly because the overwintering survival of females may be so low that they are rarely taken in light traps.

The dates of the first light trap collection of Cx. tarsalis in Winnipeg, and the rapid increase in the counts each year (Raddatz 1985) would suggest that light traps usually capture females from the first summer generation. If the median date of the first light trap collection, 6 June, is considered to be the mean emergence date for the first generation and if females acquire a blood meal by one week post-emergence, then three generations of $C x$. tarsalis are possible by the end of August (Buth 1983).

In the western United States, overwintering females of $C x$. tarsalis are nulliparous and do not seek blood meals (Bellamy and Reeves 1963; Mitchell 1981). There are several factors that indicate females of $\mathrm{Cx}$. tarsalis emerging after mid-August in Manitoba do not seek a blood-meal and would therefore not be involved in vectoring WEE virus. The numbers of females that come to $\mathrm{CO}_{2}$ traps (Brust unpublished), sentinel chicken flock traps (Brust 1982) and light traps (Brust 1982; Raddatz 1985) decrease rapidly after mid-August. Egg rafts in ovipools are most numerous during late July and early August in Winnipeg, and may be collected until early September. The population arising from these should be sizable and trap collections should remain high when evening temperatures are above the threshold for flight. If these females do not seek hosts, the August decrease in $C_{x}$. tarsalis in sentinel chicken flock traps and the $\mathrm{CO}_{2}$ traps in Manitoba is to be expected. The simultaneous decrease in the light trap collections suggests that diapausing females may also not be attracted to lights.

An alternative hypothesis to explain the rapid August decrease in adult $C x$. tarsalis counts in 1 ight, $\mathrm{CO}_{2}$, and flock traps is that there is a marked decrease in the population after mid-August. From a meteorological perspective, the annual mean daily temperature curve which normally peaks (2l C for Winnipeg) during July, drops to levels similar to mid-June (16 to $17 \mathrm{C}$ ) by late August. If $C x$. tarsalis activity levels are exponentially related to mean daily temperatures (Raddatz 1985), the seasonal drop-off of temperatures would have a significant effect on trap 
counts and therefore on population levels.

Autogeny is known to occur in field populations of $C x$. tarsalis throughout the range of this species in North America. California populations may have autogeny rates as high as 90 to 95\% (Spadoni et al. 1974) and these rates decline during the fall (McDonald 1975; Moore 1963; Reisen et al. 1983; Spadoni et al. 1974). Further study is needed to determine if the decrease in autogeny in the fall is due to the same factors that produce gonotrophic inactivity in anautogenous females (Reisen 1986).

In Ontario, during 1978 to $1980, C x$. p. pipiens entered diapause in early July, with more than $90 \%$ of the field population in diapause by mid-August (Madder et al. 1983a). Some females continued laying eggs in the field until 15 October (Madder et a1. 1980). There were three generations from May to September (Madder et al. 1983b).

iv. Interpretation of Trap Data

Various methods have been used to collect vector mosquitoes for virus isolation and to evaluate the effectiveness of vector control programs. An extensive review of mosquito sampling methods may be found in Service (1976).

The most commonly used trap for live collections of mosquitoes for surveillance of mosquito-borne pathogens in North America is the Centre for Disease Control (CDC) miniature light trap (Sudia and Chamberlain 1962), supplemented with dry ice (Newhouse et al. 1966). For the purpose of collecting infected vectors of WEE or SLE viruses the dry ice baited CDC trap collects a significant proportion of parous (potentially infected members of the population) females (Meyer et al. 1975). The efficiency of vector surveillance may be improved by combining a CDC trap with an oviposition site attractive to Culex spp. (Reiter 1983;

Surgeoner and Helson 1978).

For the purpose of estimating population density, CDC dry ice baited traps capture larger numbers of female Cx. tarsalis than New Jersey light traps (Milby et al. 1978).

Culex pipiens complex females are less readily attracted to dry ice. In Ontario, the New Jersey light trap captured as many $C x$. p. pipiens and $C x$. restuans females as the CDC dry ice baited trap (Copps et al. 1984). In the southern and eastern United States, $C x$. pipiens quinquefasciatus is even less attracted to dry ice baited CDC traps than $C x$. p. pipiens. Other collecting methods must be used such as cone or shed traps baited with chickens (Bowen and Francy 1980; Mitchell et al. 1980). Chickenbaited shed traps (Rainey et al.1962) have been used effectively to collect $C_{\mathrm{x}}$. tarsalis in southern Canada (Brust 1982; McLintock et al. 1966; Shemanchuk 1969).

Standard New Jersey light traps are commonly used by mosquito control districts in North America to monitor seasonal mosquito activity. Their primary uses are to determine where adulticiding may be needed within the district, to evaluate the level of control achieved.

The trap counts of vector species are used to assess the 
daily activity of vectors during the season and to compare their activity over a number of years. Olson et al. (1979) analyzed 21 years of light trap catches of $C_{x}$. tarsalis from 55 Mosquito Abatement Districts in California and were able to associate light trap indices (LTI = number of females per trap per night) of $C x$. tarsalis with the activity of WEE and SLE viruses.

The critical level of light trap counts of $C_{x}$. tarsalis in urban areas of California, below which no human cases of WEE or SLE were reported, was a LTI of 0.1 . Peaks in the annual incidence of WEE or SLE occurred during those years when seasonal averages in urban areas reached a LTI of 21 . Weekly incidence of human cases in urban areas was associated with a LTI of 21 for SLE and a LTI of 81 for WEE cases. In rural areas, human cases of SLE occurred when $C_{X}$. tarsalis averaged a LTI of 0.4 to 0.6 for the season. The average LTI for $C_{x}$. tarsalis must be $>0.1$ before human cases of SLE or WEE would be expected in California.

However, human cases may have been contracted when and where vector populations were much higher than the state average for the season.

The critical level for $C x$. tarsalis abundance, in regard to the epidemic spread of WEE to humans in California, was estimated to be a LTI > 10 (Reeves 1971), or a $\mathrm{CO}_{2}$ baited CDC trap index > 30 (Reisen et al. 1984). Analyses of light trap data rarely consider the environmental conditions, if known, that prevent or hinder flight activity in mosquitoes. These factors must be considered when interpreting trap counts as an index of virus transmission.

There is a threshold temperature, depending upon the species and the latitude, below which little or no flight to traps occurs regardless of the size of the resident mosquito population. In Winnipeg, Manitoba, the number of $C x$. tarsalis collected in New Jersey light traps increases as the temperature increases above 15 $C$, and decreases below that temperature (Raddatz 1985). The threshold temperature for flight in $C x$. tarsalis, on the basis of light trap collections, is about 8 to $10 \mathrm{C}$ (Brust and Ellis 1976a). On a seasonal basis, the assumption is frequently made that an equal number of warm and cool nights will occur each year and that the years can therefore be compared. However this is unlikely to occur. Analyses of light trap counts in the future should use available temperature data from the immediate area (e.g. Raddatz 1985). Any conclusions drawn made about population levels from trap counts which have not been adjusted for weather changes must be suspect.

Other environmental factors that may affect the numbers of mosquitoes collected in traps, include relative humidity or vapour pressure deficits (Clark et al. 1976), mean nightly wind speeds, moonlight, physiological state of the females (Bidlingmayer 1985), and both the specific and the general trap location (Copps et al. 1984).

The physiological state of the female mosquito (such as nulliparous, gravid, or parous) influences the collection of vector mosquitoes in different kinds of traps. Meyer et al. 
(1975) found that $\mathrm{CO}_{2}$-baited $\mathrm{CDC}$ traps collected more parous females of $C x$. tarsalis than did New Jersey light traps. However, Magnarelli (1975) found the reverse in $C_{\mathrm{X}}$. restuans, and Feldlaufer and Crans (1979) found that New Jersey light traps collected more parous $C x$. salinarius then did the same trap baited with dry ice. Resting box collections may be less biased towards any particular physiological state and the greater percentage of nulliparous mosquitoes usually found in resting boxes (Meyer et al. 1975; Nelson 1964) may reflect the natural population more accurately.

\section{b. Virus Activity}

\section{Mosquito Populations}

Recovery of WEE and SLE virus from Culex vectors is an important surveillance tool, because it determines the potential for virus transmission to humans. However, human case incidence of WEE and SLE viral infections is not always correlated with virus infection rates in vectors because the findings do not differentiate between infected individuals and infective ones (Bowen and Francy 1980). The significance of infection versus transmission rates in vectors has been reviewed by Reeves et al.(1961) and the factors influencing vector competence have been reviewed by Hardy et al. (1983), McLintock (1978) and Mitchell (1983a).

Surveillance for virus activity in mosquitoes was begun in Canada in 1942, when the Manitoba Department of Health and Welfare, in conjunction with the Children's Hospital of Winnipeg, began a study of the relationship between mosquitoes and WEE virus in Manitoba (McLintock 1946, 1947, 1948). Subsequently, surveillance for virus activity in mosquitoes has been carried out for other viruses causing human illness (McLean 1975, 1979; Mclintock and Iversen 1975; Artsob and Spence 1979).

The 1975 outbreak of WEE in Manitoba resulted in a decade of surveillance of WEE virus activity in that Province. Subsequent outbreaks were studied in more detail than the one in 1975 but not all of these have been published. Results on the 1975 outbreak can be found in Sekla (1976) and on the 1977 and 1981 WEE outbreaks in Sekla (1982). Virus activity, other than WEE, was also monitored in mosquitoes from Manitoba (Sekla et al. 1980; Sekla and Stackiw 1982). Results of the environmental studies conducted during the 1983 WEE outbreak in Manitoba are in a report by Manitoba Environment, Workplace Safety and Health (1984).

During two WEE epidemic years in Manitoba, the number of WEE virus isolations from mosquitoes ranged from 71 isolations in 1977 to 32 in 1981. Of these 33 and 18 respectively were isolated from Cx. tarsalis. During 4 non-epidemic years, the number of WEE virus isolations ranged from 0 to 4 . None of these were from $C x$. tarsalis (Sekla and Stackiw 1982). In 1977, the seasonal infection rate in $C_{X}$. tarsalis was 1:347. In 1981 it was 1:283. In North Dakota, during the epidemic year 1975, the seasonal infection rate was 1:500 Cx. tarsalis (Monath 1979).

In Saskatchewan, the infection rates of WEE virus in $C x$. 
tarsalis were a seasonal average of $1: 145 \mathrm{Cx}$. tarsalis in 1963 and $1: 169$ in 1965. Both years were outbreak years (McLintock et al. 1966; Mclintock et al. 1970). As in Manitoba during 1976 to 1981, WEE virus was not isolated from $C x$. tarsalis during non-epidemic years.

In Alberta, WEE virus was isolated from $C x$. tarsalis collected from mammalian burrows during 1965. The infection rate, calculated on the number (1141) of females of this species examined for blood, was $1: 285 \mathrm{Cx}$. tarsalis. No human cases were reported that year in Alberta but there was an epizootic amongst equines and there were human cases in Saskatchewan (Artsob and Spence 1979; Morgante et a1. 1968; Shemanchuk and Morgante 1968).

Infection rates of WEE and SLE viruses in $C x$. tarsalis may change during the season. When this occurs, the rate increase must be monitored carefully to evaluate potential risk to humans (Reeves and Hammon 1962). In Saskatchewan, McLintock et al.(1970) found that the WEE virus infection rate in $C x$. tarsalis reached a weekly high of 1:43 whereas the seasonal average was 1:145 during the epidemic year of 1963. During the 1965 epidemic year in Colorado, WEE virus infection rates in $C x$. tarsalis increased from $1: 667(1.5 / 1000)$ on $13 \mathrm{June}$ to $1: 67(15 / 1000)$ by $18 \mathrm{July}$. By 25 July, the infection rate began to decrease (Hess and Hayes 1967). The method of vector collection may affect the rate of virus recovery. More efficient methods of collection of potentially infected females need to be considered when surveillance is undertaken. A trap that combines an oviposition pool attractive to the vector species, which selectively captures gravid females, increases the chances of isolating a mosquito-borne pathogen (Surgeoner and Helson 1978). By reducing the size of the oviposition container, thereby forcing gravid females to fly closer to the suction fan, Reiter (1983) was able to capture most gravid females before they oviposited and escaped. In Memphis, Tennessee, he obtained 141 Culex females per oviposition trap night versus one female per resting box collection and 0.4 females per New Jersey light trap night.

\section{i1. Sentinel Flocks}

The use of domestic chickens to monitor WEE and SLE virus activity has been widely adopted in North America (McLintock et al. 1966; Rainy et a1. 1962; Reeves and Hammon 1962; Wong et a1.1976).

Chickens infected with WEE virus become viremic within 1 or 2 days, remain infective for 2 or 3 days and then become immune (Bellamy et al. 1967; Wong et al. 1976). Sentinel chickens are usually bled every two weeks, allowing sufficient time for antibodies to develop to detectable levels (Wong et al. 1976). To be an effective early warning of an impending epidemic, chickens should be placed on location as soon as vectors appear in the spring. Sentinel chickens that develop antibodies should be replaced immediately with new birds to maintain the flock size, exposure rate and attraction to mosquitoes remains at a constant level (Bowen and Francy 1980). 
Wild birds

Wild birds are frequently surveyed to establish antibody prevalence to WEE and SLE viruses (Bowen and Francy 1980; Burton et al. 1966; Burton and McLintock 1970; Dorland et al. 1979; Hayes et al. 1967; Hess and Hayes 1967; Holden et al. 1973; Reeves and Hammon 1962). If only juvenile birds are sampled or if juveniles are carefully separated and the results are tabulated separately from those from the adults, the data will more accurately reflect viral activity for the year. Sparrows are most frequently collected using mist nets. Repeated samples, weekly or biweekly, of 100 to 150 birds from several sites are necessary to provide an adequate early warning of viral activity in a geographic area (Bowen and Francy 1980). Meaningful assessment of antibody levels depends upon the availability of background data from previous epidemic and non-epidemic years, and upon serial measurements from spring to fall. A sudden or gradual increase in antibody level in juvenile birds may be highly significant (Bowen and Francy 1980).

\section{iii. Reservoirs, Indicators}

Vertebrate reservoirs may play three important functions in epidemiology of mosquito-borne viruses. They may serve to amplify virus levels in spring as capable vector species first become active. They may serve as maintenance hosts for the virus throughout the summer and a pool of virus available for vectors to spread the virus to other epizootic or epidemic hosts. Finally, as rates of transmission decrease in autumn with reduced vector activity, the vertebrates may be overwintering reservoirs for the virus.

If wild vertebrates, either birds or mammals or both, are critical to initiation of virus introduction, maintenance and spread, then intuitively, they might be used effectively in a surveillance program as an early warning system for possible disease outbreaks. Limited serosurvey work for mosquito-borne virus activity has been done in Canada. Meaningful surveillance of wild vertebrates must rely on a clear understanding of the specific vertebrates involved, and their roles as they affect distribution and abundance of the virus. Information must be available on reproductive patterns, exposure and availability to vector mosquitoes, response to virus infection, serological response following active infection, and patterns of movement (dispersal and migration). Trained personnel are required in the field to collect the appropriate verebrate species and to take blood samples. Extensive data are necessary for several out break and nonoutbreak years before critical levels of virus activity in vertebate populations can be associated with pending outbreaks.

Only one mosquito-borne virus warrants consideration of wild vertebrate surveillance in Canada, viz Western Equine Encephalitis Virus. Eastern Equine Encephalitis Virus and the known Californian Group Viruses are not prevalent enough to justify extensive surveillance. There has been only one epidemic of $S t$. Louis Encephalitis, in Ontario in 1975, and although antibodies 
have been detected in both resident English sparrows, and migratory passerines (see Artob and Spence 1979), extensive effort to monitor wild birds seems unjustified at the present time.

The history of Western Equine Encephalitis Virus is marked by epizootic outbreak years in the Prairie Provinces. Wild vertebrates have been examined for evidence of virus infection and many species have been implicated. Antibodies have been detected in ducks, blackbirds, pigeons, English sparrows, barn swallows, starlings, ruffed and sharp-tailed grouse, Swainson's hawks, gulls, magpies and robins (Burton et al. 1961; Burton et al. 1966b). Seropositive mammals include Richardson's and Franklin's ground squirrels, snowshoe hares, red foxes, skunks, reindeer, bison, moose, pronghorn antelope, red-backed voles, weasels, and muskrats (Burton et al. 1966b; Gwatkin and Moynihan 1942, Leung et a1. 1975; Yuill and Hanson 1964; Burton and McLintock 1970; Trainer and Hoff 1971; Barrett and Chalmers 1975; Hoff et al. 1970; Sekla and Stackiw 1982). Virus and neutralizing antibodies have also been isolated from garter snakes and leopard frogs (Burton et al. 1966a; Spalatin et a1. 1964; Prior and Agnew 1971). These numerous isolations were, however, detected in trying to establish the existence and/or role of wild vertebrates in the western equine encephalitis cycle. Until these factors are known, surveillance activity for wild vertebrates in Canada will be inefficient or of little value.

Western Equine Encephalitis Virus outbreaks have been unpredictable in time and space. Even by concentrating on species of vertebrates in which levels of virus activity may be an indication of later season activity (e.g. English sparrow - Hess and Holden 1958), the amount of effort and expenditure of resources may not be justified on the basis of the return. More valuable information would be generated by placement of sentinel vertebrates (e.g. chickens or equines) in strategic locations. Sentinel animals are more easily accessed, require fewer blood samples to be processed, and have a known history of virus exposure and antibody response.

In conclusion, it is apparent that it is not feasible to introduce wild vertebrates into arbovirus surveillance programs in Canada at the present time. Unless we gain additional information on the role of wild vertebrates in the virus cycle, and unless there is evidence that certain species provide better correlation of virus activity and disease outbreaks than existing techniques, implementation of vertebrates as a surveillance tool is unwarranted.

\section{c. Criteria}

1. Mosquito Counts

When WEE or SLE viruses are detected in vector mosquitoes, the size of the vector population is an important factor in the spread of these viruses to humans (Brust 1982; Mclintock et al. 1966; Reeves 1965; Reeves and Hammon 1962). The most common method of determining the relative size of the mosquito population and the mosquito species in an urban area, is the use of the New 
Jersey light trap. Live trapping of mosquito vectors for the purpose of virus isolation may be accomplished by using a modified New Jersey type light trap (Brust 1982; McLintock 1946) but usually the CDC light trap baited with dry ice is used. The counts from each of these trap types may used to assess the relative population size during the season or from year to year. For $C_{x}$. tarsalis counts in California, a dry ice-baited CDC trap index of 30 females per trap night, compares to a New Jersey light trap index of about 10 per trap night (Milby et al. 1978; Reisen et al. 1984).

New Jersey light trap collections have been used to estimate minimum levels of abundance of $C x$. tarsalis females associated with WEE and SLE viruses in humans in California over the period 1953 to 1973 (Olson et al. 1979). The LTI was calculated for both rural and urban areas. Because most abatement districts operate New Jersey light traps in urban areas, with a few rural sites, the LTI's from urban areas in California may be of greater value in assessing vector threshold levels of this species in the future. On a seasonal basis in California, the critical level of $C x$. tarsalis counts, below which no human cases of these viruses were detected, was a LTI of 0.1. However critical levels of females associated with a significant number of human cases were between a LTI of 6.4 and 62.4. Peaks in the weekly incidence of SLE cases were associated with a LTI of 21 and of WEE cases with a LTI of 81 (Olson et al. 1979). A LTI for $C x$. tarsalis of $>10$ is considered to be the critical level for WEE virus transmission to humans in California (Reeves 1971; Reisen et al. 1984).

Mosquito counts from sentinel chicken shed traps, each containing 10 birds, have been used to calculate population levels of $C x$. tarsalis associated with epidemic years in Manitoba (Brust 1982). Both the numbers and the $\% C x$. tarsalis in the weekly collections were correlated with epidemic years. During epidemic years, > 20 females per trap week were collected during the last half of June and the first half of July. The threshold level for \% Cx. tarsalis in the collections during this period was $25 \%$. The number of and the $\% C_{x}$. tarsalis in sentinel chicken shed trap collections were considerably below those levels during nonepidemic years (Brust 1982).

Seasonal light trap counts of $C_{x}$. tarsalis in Saskatchewan, using a modified New Jersey live trap, were higher during the epidemic year of 1963 than during the non-epidemic year of 1964 (McLintock et al. 1966). McLintock previously used this trap in Manitoba during 1942 to 1948 and found that the $C x$. tarsalis counts were highest during the epidemic year of 1947 (McLintock and Rempel 1963).

An analysis of 7 years of $C x$. tarsalis counts (1977 to 1983) in New Jersey light trap collections from Winnipeg, Manitoba, was conducted by Raddatz (1985). Also a forecast count and an actual count was made for 1984. Two epidemic years had higher levels of $C x$. tarsalis than 4 non-epidemic years. However, 1 epidemic year (1981) had lower counts than 2 of the non-epidemic years (1978, 1984) (Raddatz 1985), demonstrating that there may be exceptions 
to the association of WEE human cases with abundance of $C_{x}$. tarsalis.

\section{Significance of Seroconversion}

Seroconversion in sentinel chickens is usually correlated with the appearance of human cases of SLE and human and equine cases of WEE (Bowen and Francy 1980; McLintock et al. 1970). In Manitoba, the increase in seroconversion in sentinel chickens occurred simultaneously with the increase in equine cases in 1975 (Lillie et al. 1976; Wong et al. 1976). Human cases followed the equine cases by about 2 weeks (Waters 1976). However, human cases may not follow low, background levels of seroconversion.

The level of seroconversion in sentinel chickens in Manitoba reached 50\% during 1975 and $84 \%$ during 1981 (Wong et al. 1976; Wong and Neufeld 1982). Seroconversion rates $>20 \%$ per exposure period indicates high viral activity and, according to Wong and Neufeld (1982), can lead to an epizootic and an epidemic of WEE.

In Saskatchewan, WEE virus seroconversion rates in sentinel chickens averaged $42 \%$ in 1963 and peaked at $92 \%$ by the end of August (McLintock et al. 1966). In Kern County, California, the highest seroconversion rate (50\%) to WEE virus in chickens over a 10 year period (1943 to 1952) was associated with the epidemic year of 1952. The seroconversion rate exceeded $20 \%$ ( 26 to $34 \%$ ) in rural areas, where rates were higher than in urban areas, for 4 other years; the number of human cases of WEE during those years was greater than average in 1943 and 1945 but lower than average in 1949 and 1950 (Reeves and Hammon 1962).

During SLE epidemics in the United States, seasonal seroconversion rates in chickens have varied between 30 and $90 \%$ (Bowen and Francy 1980). However, one human case of SLE occurred in Kern County, California, when the seasonal seroconversion rate in chickens averaged only $2 \%$ (Reeves and Hammon 1962).

\section{Weather Factors}

Summer temperatures and precipitation may influence the size of vector populations such $C x$. tarsalis (Fraser and Brust 1976; Raddatz 1985; Reeves and Hammon 1962). Wet spring conditions, followed by warm temperatures, have been associated with unusually rapid increases in $C x$. tarsalis populations in Manitoba and California.

Summer temperatures may affect the extrinsic incubation period of arboviruses, the transmission to vertebrate hosts and the extent of their geographic distribution (Hess et al. 1963). In California, WEE virus was detected in mosquitoes after temperatures exceeded $26 \mathrm{C}$ for 2 weeks (Reeves and Hammon 1962). When warm temperatures occurred earlier in the summer, during years when WEE virus was present, virus activity occurred earlier in California. When cool spring temperatures prevailed, virus activity was retarded.

In Saskatchewan and Manitoba, the temperatures suitable for extrinsic incubation of WEE virus may be lower than those in more southern regions. Temperatures rarely exceeded $26 \mathrm{C}$ in 
Saskatchewan in 1963 or in Manitoba during 1975. Weekly means never reached $26 \mathrm{C}$ in Manitoba during either 1975 or 1981 (Fraser 1982; Fraser and Brust 1976; McLintock et a1. 1966). The threshold temperature for extrinsic incubation of WEE virus in $C x$. tarsalis is probably about 19 to 21 C (Mclintock et al. 1966). This would agree with the association of WEE epidemics with the geographic region above the $21 \mathrm{C}$ June isotherm in North America (Hess et al. 1963).

SLE epidemics are associated with geographic regions of North America that have a mean temperature of at least $21 \mathrm{C}$ during June (Hess et al. 1963; Monath 1980). Only 1 SLE epidemic occurred in Canada, and this was limited to southern Ontario, a few hundred $\mathrm{km}$ north of the $21 \mathrm{C}$ June isotherm. Temperatures during 1975 were above normal during May, June and July, and may have played a role in the northern spread of SLE that year (Bristow 1979). In California, SLE isolations have occurred later in summer than those of WEE and have followed periods when temperatures exceeded $28 \mathrm{C}$ (Reeves and Hammon 1962).

Temperature affects the development and abundance of vector populations that transmit WEE and SLE viruses. This in turn affects the spread of these viruses to vertebrates during the spring amplification cycle and the spread to humans and other hosts during the summer months (Hess and Hayes 1967; McLintock et al. 1966; Reeves 1965). In Manitoba, a weekly mean temperature of $16 \mathrm{C}$ is significant in the development of $\mathrm{Cx}$. tarsalis. Below this temperature, development is retarded or halted (McLintock 1948). According to McLintock (1948), two other factors are important for $C_{x}$. tarsalis to become abundant in Manitoba 1) the average mean weekly temperature during the latter half of June must be above normal, and 2) the average mean weekly temperatures for July and August must be $2 \mathrm{C}$ above normal. However, during 1975 and 1981 in Manitoba these conditions were not met (Fraser and Brust 1976; Fraser 1982). New criteria are needed to determine which weather factors are important in the increase of $C x$. tarsalis populations and the transmission of WEE virus to vertebrates.

Daily mean temperatures below $15 \mathrm{C}$ have been shown to be important in reducing trap collections, and therefore flight in Cx. tarsalis in Winnipeg, Manitoba (Raddatz 1985). Based on 7 years of light trap collections, Raddatz (1985) was able to model the effect that temperature, precipitation, evapotranspiration and runoff had on collections, and presumably on population size, of $C x$. tarsalis in Winnipeg. The effect other weather factors have on trap collections (e.g. wind, relative humidity, and vapour pressure deficit) are reviewed by Bidlingmayer (1985).

d. Provincial Surveillance

1. Western Equine Encephalitis

Epidemics of arboviruses occur when vector abundance and transmission of virus exceed thresholds at which the virus is carried over into human and equine hosts. Arbovirus surveillance 
programs entail monitoring of adult populations of vector species, and virus transmission rate, virus isolations, and diagnosis of infections in natural and dead-end (including human) hosts.

Provincial surveillance programs in Canada were reviewed in 1980 - 1981 as part of the feasibility study for the Canada Biting Fly Centre (Appendix IV). At that time, annual surveillance programs for Western Equine Encephalitis were carried out in Manitoba and Saskatchewan. Since then, the Manitoban program has been terminated and the Saskatchewan program reduced. No annual programs are carried out in Alberta, British Columbia, the Maritimes, nor the Territories. Incidence of encephalitis infection in humans in recent years in these regions was nil.

No other annual surveillance programs, except that in Ontario for SLE, are carried out in Canada. Human incidence of EEE infections have occurred in Quebec, but at levels too low to justify the expense of annual surveillance. The National Arbovirus Reference Centre in Toronto monitors incidence of human infections.

Surveillance is carried out in the United States for several arboviral diseases, including WEE, EEE, and SLE, in the states bordering Canada ${ }^{1}$. These jurisdictions provide further input into Canadian surveillance programs, as well as an alert for the implementation of ad hoc surveillance by Canadian authorities.

\section{Manitoba}

Manitoba began surveillance in 1976, after the outbreak of WEE in 1975. The program was carried out by Manitoba Health, and co-ordinated, initially, by the Manitoba Arbovirus Surveillance Committee (1975-1983) and subsequently by the Western Equine Encephalitis Surveillance Committee (1984-1985). Both committees were made up of multi-agency, multi-disciplinary members who were scientists or other health professionals. The program was carried out to predict outbreaks of WEE in the human population and to recommend remedial action. Following an outbreak and extensive emergency mosquito control program in 1983, the fourth in seven years, the Manitoba Government reviewed both the efficacy and environmental monitoring of its emergency aerial spray program, and, separately, mosquito control in the province. The outcome of the review was a re-assessment of provincial procedures and requirements for WEE surveillance and control. The Province maintained its commitment to surveillance and assessment of risk of outbreaks, but rated emergency aerial spray measures as a strategy to protect the public as low priority. Control measures, if warranted, were to be focused on public advisories and personal protection. In 1986, the Government withdrew financial support for the program and surveillance was discontinued in April, 1986.

${ }^{1}$ Ohio Vector Borne Disease Unit, Ohio State Department of Health; Mosquito Research and Control Group, Minnestota State Department of Health; Division of Environmental Sanitation, North Dakota State Department of Health. 
The Province of Manitoba developed the most intensive arbovirus surveillance program in Canada. It consisted of monitoring of weather, abundance of mosquito populations (including $A e$. vexans, Cs. inornata, Cx. restuans, as well as the primary vector, $C x$. tarsalis), virus activity as indicated by isolations from mosquitoes, infections in sentinel flocks, and horse and human infections (See CRITERIA 5a). The program also included exchange of monitoring data or assessments with neighbouring jurisdictions. The Manitoba Arbovirus Surveillance Committee (MASC) was comprised of specialists in medical entomology, epidemiology, veterinary medicine, virology, environmental management, insect control, meteorology, and public health. The mandate of the Committee was to determine risk of outbreak of disease, and to inform the Manitoba Minister of Health.

The provincial program generated criteria for assessing risk on a province wide basis. Surveillance in Saskatchewan has been carried out for longer, but at a smaller, localized scale. Comparisons of programs are difficult because of differences in level of surveillance, approach to surveillance, and approach to protection of public health. Criteria developed in Manitoba were used to assess risk to public health and were also used in decision making on emergency aerial spraying of municipalities.

\section{Saskatchewan}

The WEE surveillance program is part of an annual arboviral surveillance program begun over 30 years ago. The program was a joint venture between the University of Saskatchewan (Department of Veterinary Microbiology) and the Agriculture Canada Research Station; now it is carried out by the University. The program consists of monitoring mosquito populations and virus activity(see CRITERIA 5a). Mosquito populations are monitored using New Jersey style light traps in three selected sites, and virus activity is assessed by monitoring seroconversion rates sentinel chicken flocks and incidence of horse cases. The extensive database gained over three decades of monitoring and research enables experienced scientists to predict likelihood of outbreaks in some local areas.

\section{Ontario}

The province does not carry out routine surveillance for WEE, but keeps in regular contact with neighbouring jurisdictions for prediction of potential outbreaks. Incidence of WEE in Ontario is low; it was first recognized in the western region by horse infections in 1981, concurrent with an outbreak in Manitoba. WEE is not known to occur in provinces east of Ontario.

\section{Other Jurisdictions}

Local mosquito abatement authorities in Canada are too small and too scarce to provide a major component of surveillance and 
control in province-wide surveillance programs. Some local authorities, e.g., in Manitoba the City of Winnipeg and the City of Brandon have the expertise and, in Winnipeg, the resources to make significant contributions, but this level of support is not typical.

Following cancellation of the provincial program in Manitoba in 1986, the Insect Control Branch of the City of Winnipeg, concerned for its mandate to protect Winnipeg residents (representing over $50 \%$ of the provincial population) from WEE, implemented its own surveillance program. The program consists of monitoring virus activity both in mosquito populations and sentinel flocks on a reduced scale to that of former provincial program. The City routinely monitors mosquito populations with a system of 25 New Jersey style light traps within the City's control zone and additional traps outside the control zone. Five sentinel flocks traps of the design used by the province (Wong et al. 1976, Wong and Neufeld 1982) are used in selected sites located across the City. Mosquito pools and sentinel flock blood samples, collected biweekly, are analyzed by the provincial public health laboratory.

The City of Regina mosquito control program has two stated main objectives: to prevent major mosquito infestations from developing in breeding sites, and to monitor populations and identify various mosquito species present throughout the season, in particular those vectors of Western equine encephalitis (Ann. Rpt. Mosq. Control, City of Regina, 1988)

In Alberta and British Columbia, population abundances of Cx. tarsalis are monitored through local control operations. Municipal mosquito control programs in both these provinces are regulated by provincial authorities. Emergency monitoring of mosquito populations and virus activity are carried out when concern of outbreaks is raised as a result of high populations of vector species and identification of viral infections either in the provinces or in adjacent jurisdictions.

\section{Problems in Surveillance Programs}

Problems identified in Manitoba in carrying out surveillance and assessing risk of human infection stem in part from the decision-making process and from the options for remedial action, once the risk of outbreak was identified; and partly from shortcomings in surveillance procedures. Because of the low number of expert local control organizations and the inadequacies of larviciding for emergency measures, the Province resorted to emergency control measures ${ }^{2}$ during each of the last four

2 Control approaches followed in California, where surveillance and control programs have been carried out for 30 years (Walsh 1987) and which represent the best available technology, are not applicable to Canada because of less severe health consequences and lower socioeconomic impacts of mosquitoes in Canada. 
outbreaks. This history of reliance on emergency aerial spraying influenced decision making by the Committee.

The decision-making authority to implement emergency control measures rests with the Minister(s) of Health. As a result of the Committee's role in developing the surveillance program, developing criteria to asses risk, and carrying out the program and assessing risk, the Manitoba Arbovirus Surveillance Committee was a major influence on decisions to implement emergency aerial spraying. The decision-making process lacked adequate separation of stages between identification of risk of human infection and the decision to implement emergency aerial spraying.

Furthermore, effectiveness of remedial action is doubted: the 1981 emergency aerial spray was estimated by provincial officials to provide only $10 \%$ protection at best for the public (unpubl. Inter-Departmental Review Review Committee, Manitoba Government 1984). Failure to achieve greater protection was considered primarily due to lack of exposure of $C x$. tarsalis populations to adulticide because ULV aerial spraying does not penetrate well into resting sites and because federal regulations prohibit aerial application of insecticides during nightfall, i.e., during peak $C x$. tarsalis activity periods. The effectiveness of emergency aerial spray in reducing incidence of WEE cannot be determined with precision; it can only be inferred based on differences in mosquito counts.

The severity of WEE as threat to public health is also doubted. The lack of large numbers of either horse or human cases in outbreaks in Manitoba since the $1960^{\prime} \mathrm{s}$ is indicative of a change in pattern of the disease. One possible explanation is a loss of virulence of the WEE virus occurring in the Canadian prairies. A second is the change in land use, for example, with increased urbanization in the southern regions of Canada, improved agricultural irrigation, and flood control systems, all of which have contributed to reductions in the extent of breeding sites. Considered in conjunction with changes in lifestyles (Gahlinger, Reeves and Milby 1986), these changes have led to reductions of human exposure to the virus.

Jurisdictions differ in attitude and approach to WEE as a threat to public health. The conditions considered necessary for outbreak of WEE in Manitoba have also been present concurrently in Alberta, and British Columbia, yet these provinces did not implement emergency aerial spray measures. No outbreaks occurred there, even though no large scale aerial spraying was carried out. Public and government attitude to WEE in these two provinces differ than that in Manitoba.

Problems in assessing risk were also a result of variables ensconced in the surveillance procedures:

Mosquito Trapping

The variety of trapping techniques used to assess population abundances necessitated different interpretations of trap counts and differential emphasis on trap catches. For example, the program made use of NJLT data generated by three different 
jurisdictions (the Province, the City of Winnipeg, and the City of Brandon) each employing its own modification of NJLT style trap. Difficulties in trap catch interpretations were also compounded by differences in trapping schedules, and trap strategy. The surveillance program used one set of mosquito traps to monitor both mosquito populations as well as to capture large enough samples of mosquitoes for viral analysis. Flock traps, 1ight traps, and $\mathrm{CO}_{2}$-baited traps capture mosquitoes differentially, according to mosquito species, age, behavioural activity, as well as ambient (i.e. temperature, humidity and light intensity) conditions.

\section{Equine Cases}

Lack of data on frequency and extent of horse vaccinations, and lack of information on horse cases compromise the value of a relatively reliable indicator of impending human infections. By the time horse cases are identified, it may be too late to mount an effective emergency spray program, particularly one which is monitored for environmental impacts. It is also too late to take any action other than emergency spraying and public advisories on self-protection.

Failure to Apply all Criteria in Assessing Risk

Prior to 1981, the criteria on which the Committee based its decisions were poorly defined, in part because of an inadequate data base. After the 1981 emergency, the Committee developed more formal criteria using four indicators (see CRITERIA 5a). However, these criteria were not followed consistently in 1983. That year, an initial assessment of high risk was based only on an unexpected early seroconversion of sentinel flocks. High mosquito numbers, virus in mosquitoes, and horses cases appeared later.

The practice of hiring university students as seasonal assistants has led to gaps in the database for significant periods, e.g., April and late August and September. Surveillance is not carried out at times when data are still needed to assess threat of infection in late season, and to clarify virus cycle in late summer, early fall.

\section{St. Louls Encephalitis}

Ontario is the only province in which significant SLE activity and human cases have been recorded. Following the only reported outbreak in 1975, a document was prepared by the Committee on Programs for the prevention of Mosquito-borne Encephalitis, for the Ontario Ministry of Health (Mahdy et al. 1979). In that report, provincial surveillance activities were described for mosquitoes (Helson et al. 1979b; Thorsen et al. 1979), sentinel chicken flocks (Helson et al. 1979a), wild birds (Dorland et al. 1979), horses (Artsob et al. 1979), and humans (Mahdy et al. 1979). 
a. Western Equine Encephalitis

The objective of arbovirus surveillance programs is to predict the risk of transmission of virus to the human population in time to implement effective remedial action. Remedial measures continue to focus on suppression of vector population abundance, primarily by chemical control as part of integrated pest management programs. However, authorities relying on current vector control strategies are facing increasing opposition to pesticide use because of the environmental and public health implications of adulticiding, and, with respect to WEE, scepticism over the effectiveness of adulticiding in reducing human infections.

McLintock (1976) described conditions necessary for an outbreak of Western Equine Encephalitis (WEE) to occur.

1. There must be susceptible population. For WEE as well as SLE, horses and humans are susceptible populations. Rural residents are more at risk than urban ones (in Reisen and Monath 1988, Eadie and Freisen 1982).

2. The virus must be available to the mosquito vector. Infected passerine birds are the most important source of virus for the mosquito. Since the life cycle of WEE virus is not fully known (see DISEASES, 2a; VECTORS 3a), the precise role, as reservoirs, of birds and mammals from which virus isolations have been made is not well understood. Horses and humans, on which infected mosquitoes feed after fledgling birds are no longer readily available as hosts, are normal hosts of mammophilic mosquitoes infected with the virus.

3. Populations of mosquito vectors must be sufficiently large to sustain an epidemic. Cx. tarsalis is the only species known to transmit WEE virus to humans and horses.

Surveillance programs monitor parameters leading to the second and third of these conditions. The first condition is taken into consideration in determining control strategy. Criteria developed by the Manitoba Arbovirus Surveillance Committee were used to assess risk to public health in Manitoba and were also used in decision-making on emergency aerial spraying of municipalities. They were based on data collated by McLintock for Saskatchewan, and experience gained in Manitoba since the 1975 outbreak.

Prior to 1981 , the criteria were not precisely defined because of a lack of an adequate database. Following criticism and review of its handling of the 1981 outbreak, Manitoba Health attempted to devise more rigorous criteria using four indices. However, these criteria were not followed consistently in 1983, the next period of increased viral activity and a subsequent outbreak.

Assessment of risk was patterned after the surveillance programs in Saskatchewan and California (Walsh 1985) and were developed by comparisons between outbreak and non-outbreak years. The Manitoba Arbovirus Surveillance Committee developed thresholds of risk based on trap data, incidence of seroconversion in 
sentinel flocks, and incidence of equine cases. Thresholds were based on trap distribution and collection regime and reporting procedures used 1976 - 1981 (Brust 1982, Wong and Neufeld 1982, Sekla and Stackiw 1982). In both risk assessment and decisionmaking on control measures, indications in neighbouring jurisdictions were also considered.

Comparison of criteria between jurisdictions is difficult because of differences in surveillance programs, e.g., in trapping schedules, handling and storage of samples, climatic differences, in addition to differences in species or strains of vectors, virus, or hosts. There are also considerable differences in attitude to risks to public health and approaches to protecting public health (see SURVEILIANCE, $4 \mathrm{a}$ ).

The surveillance and decision-making processes in Manitoba led to the declaration of four public health emergencies, for each of which a wide scale emergency aerial spray program was carried out. The criteria used are the basis for this discussion.

\section{Weather}

Climatic factors used to predict outbreak conditions include temperature, rainfall, soil moisture conditions of the previous fall, winter temperatures, amount of snow and rate of snowmelt.

Temperatures are monitored to predict potential of virus multiplication in $C x$. tarsalis and other mosquitoes, and on the rate of growth of mosquito populations. McLintock (1948) described two conditions of temperatures necessary for WEE outbreaks in the prairies: a mean average weekly temperature above normal during the last two weeks of June, and average mean weekly temperatures in July and August at least $2 \mathrm{C}$ above normal. The second condition was not met in Manitoba in 1975 (Fraser and Brust 1976).

In an attempt to forecast population abundance of $C x$. tarsalis in southern Manitoba, Raddatz developed a model for $C x$. tarsalis populations of Winnipeg, based on the City of Winnipeg's NJLT collections 1977 - 1981 (Raddatz 1982). The model, based on temperature and rainfall conditions and still requiring confirmation, offers prediction eight weeks in advance of high Cx. tarsalis counts. A warm and wet late April and early May is required for the rapid increase of $C x$. tarsalis populations in late June/early July, an increase necessary for an outbreak.

In the United States, a computerized weather monitoring program (IPM program) is being developed. The Raddatz (1985) model is an encouraging first attempt for Canada, but until it is adequately tested, it remains an experimental tool.

Winter temperatures, snow fall, and soil saturation levels are early season environmental conditions used to assess outbreak potential. These early season factors provide only secondary qualitative information. Impacts of snowfall and soil saturation levels are indicative of abundance of breeding sites, and in themselves are insufficiently direct to provide reliable predictions of virus activity.

Winter temperatures affect population abundance of 
overwintering adults. Survival of overwintering populations, including $C x$. tarsalis, is inversely proportional to mean maximum winter temperature. Successful emergence is proportional to mean monthly temperature in late spring and abundance of females entering hibernation the previous fall (Eldridge 1987b). Rapid increase in temperature and consequent melting of the snow pack lead to high populations of $C x$. tarsalis (Reisen and Monath 1988). These conditions also lead to high populations of $C s$. inornata which are assummed to have a significant role in the WEE cycle in early spring and late fall. In California, river flooding is associated with high populations of $C x$. tarsalis, but has not been a consideration in development of criteria for risk assessment in Canada.

Weather preceeding arrival of migratory birds is critical for the development of outbreak conditions (Eldridge 1987b). When local Culex populations have emerged from hibernation by the time migratory birds arrive, they are exposed to available virus in infected migrants. Adult mosquito populations active at the critical time period make an outbreak possible. SLE was introduced into Ontario in the spring of 1975 by migrating birds which are viremic for only 3 days. Time of emergence of overwintering $C x$. tarsalis populations is determined by temperature (Eldridge 1987b); survival of emerging populations is also dependent on adequately high temperatures and adequate rainfall to support a breeding population.

In Manitoba, $C x$. tarsalis does not emerge early enough to feed on northward migrating birds. Birds migrate north in April; Cx. tarsalis females typically are not trapped in Manitoba or Saskatchewan until the second or third week of June.

Abundance of Culex tarsalis Populations

Criteria for the assessing risk on the basis of mosquito abundance in Manitoba were developed from monitoring carried out using standard light traps and flock traps since 1976. Two basic trap types were used: flock traps and New Jersey Light Traps (NJLT). A third trap type, the CDC style carbon dioxide-baited trap, was also used also provide an indication of mosquito abundance because these provide large numbers of mosquitoes for viral analysis. Their value as indicators of abundance was restricted by an inadequate database on their trapping characteristics in Manitoba.

Four species of mosquitoes were monitored in Manitoba; $C x$. restuans, $C s$. inornata, and $A e$. vexans as well as $C x$. tarsalis. In Saskatchewan, additional Aedes species are monitored. Only $C x$. tarsalis is included in criteria for assessing risk of outbreaks. All selected species were tested for virus infection.

Trap catches were interpreted on the basis of trap type, frequency of trap catch collection, number and location of trap sites, and meterological conditions over the trapping period. Because mosquito activity varies widely with ambient conditions, trap counts also vary widely with ambient conditions (Bidlingmayer 1985). Hence, whenever possible, daily catches of mosquitoes were 
made. However, because of limited resources, trap catches were made at lower and various frequencies: 1 (for distant sites), and 2 , or 4 times weekly, depending on distance from Winnipeg.

\section{Flock Traps}

Flock trap design and use is described in Wong et a1. 1976, and Wong and Neufeld 1982. Initially five, and after the 1979 outbreak, eleven flock traps were used. Flock traps do not collect as many mosquitoes as light traps. Nevertheless, from data collected 1976-1981 on one flock trap and one suction style NJLT at each of five rural locations, Brust (1982) concluded that flock traps provided the most efficient indication of risk; flock trap catches showed increases in $C x$. tarsalis populations one to two weeks earlier than NJLT catches (Brust 1982).

During years when human infections occurred, flock trap catches exceeded 75 female $C x$. tarsalis /trap/week and $C x$. tarsalis exceeded $38 \%$ of the total catch of mosquitoes. In nonoutbreak years, $C x$. tarsalis counts were less than 20 females/trap/week, and less than $25 \%$ of the total flock trap catch. In outbreak years, $C x$. tarsalis populations increased rapidly before mid July. High population abundance of $C x$. tarsalis by mid July are apparently needed for virus amplification to build up to outbreak levels. In outbreak years, $C x$. tarsalis numbers increased rapidly in flock traps during the first two weeks in July or earlier: greater than $10 \mathrm{Cx}$. tarsalis /trap night for three consecutive nights by the end of the second week of July, and 20-30 Cx. tarsalis or more/trap night during the second and third weeks of July for three days of longer.

\section{Light Traps}

Light traps give an index of relative mosquito abundance and provide greater numbers of mosquitoes for viral analysis. New Jersey style light traps, modified ("suction style") for livetrapping (Brust 1982), were equipped with 100 watt bulbs rather than the standard 25 watt bulb in order to increase the number of mosquitoes caught for viral analysis. Only one NJLT was used per location, and at only 5 locations across the province.

NJLT counts of $C x$. tarsalis are also higher in outbreak years but the correlation between higher NJLT counts and outbreak years is less than that between flock trap counts and outbreak years (Brust 1982). Again, counts of Cx. tarsalis in NJLT catches of three or more/trap night by the end of first week in $\mathrm{July}$, and more than $10 \%$ of the total catch during the fourth week of June to mid July is also indicative of an outbreak.

Cx. tarsalis counts in light traps exceed those in flock traps toward the end of the season. In Saskatchewan, survival of the overwintering population is estimated on NJLT catches.

Emergence of hibernating females in spring is determined by light trap catches. If no females are caught before the first males, the hibernating population was small and survival also small.

The City of Winnipeg has maintained a network of New Jersey 
style light traps as part of its annual mosquito control program. The trap network has been operated for over a decade and now provides a useful database on mosquito population abundance. The City has developed a threshold of $C x$. tarsalis abundance based on nightly average trap catches to predict risk of human infection (Dr. R. A. Ellis, pers comm.). An average of 50 or more female Cx. tarsalis /NJLT/night is predictive of an outbreak.

Carbon Dioxide Baited Traps

CDC style light traps baited with carbon dioxide were used in 15 sites across the province between $1982-1985$. The sites were chosen on the basis of human population and history of virus activity. Three traps were used per location to reduce effects of local and ambient conditions on trap efficiency. $\mathrm{CO}_{2}-\mathrm{CDC}$ trap catches were usually consistently higher (e.g. an average of approximately $75 \%$ greater numbers of $C x$. tarsalis /trap/night in 1983 in sites where flock traps were high). However, since $\mathrm{CO}_{2}$ CDC trap data are available for only one outbreak year (1983), the data are inadequate for development of predictive thresholds.

Following the 1983 outbreak, thresholds of risk of 'low', 'medium', and 'high' of mosquito activity were determined by both flock trap and light trap catches (average number of females caught/trap/week). Greater emphasis was placed on numbers rather than on relative abundance of $C x$. tarsalis trapped and on abundance of $C x$. tarsalis at critical times during the season.

Virus Activity

Virus activity was monitored using three parameters: virus

isolations from mosquitoes, seroconversion in sentinel flocks, and incidence of horse infections. Virus isolations from mosquitoes were usually made each year, however, the rate of infections among mosquito pools differ between outbreak and non-outbreak years. During 1975-1981, less than $2 \%$ of pools of all mosquitoes species tested were positive in non outbreak years as compared nearly $5 \%$ or greater in outbreak years. Pools of Cs. inornata and $A e$. vexans, and less frequently, $C q$. perturbans, Ae. pionips, and $A n$. earlei, were among those from which WEE virus was isolated. The rate of virus isolation from $C x$. tarsalis pools was consistently greater in outbreak years: $8.5 \%$ or more of $C x$. tarsalis pools with positive whereas the virus was not found in any $C x$ tarsalis pools in non-outbreak years (Sekla and Stackiw 1982).

Sentinel Flocks

Sentinel flocks are a widely-used, valuable indicator of virus activity because they become infected when rate of epizootic transmission is high, and the virus is carried over from wild passerine hosts. When bled biweekly, sentinel flocks frequently provide seroconversion data prior to the onset of equine infection (Hess and Hayes 1967), a situation that was usual in Manitoba but did not occur in 1983.

In Manitoba, sentinel flock data gathered between 1975 and 
1981 were consistent in distinguishing outbreak years from nonoutbreak years, although the trapping system was varied in trap number, design, and location; types of birds; bleeding and exposure schedule (Wong and Neufled 1982). In non-outbreak years over the period of 1975 - 1981, the percentage of birds which seroconverted ranged from a low of less than $1 \%$ to $3.3 \%$. In outbreak years, the percentage which seroconverted was $16 \%, 26 \%$ and $84 \%$ (Wong and Neufled 1982).

\section{Equine Cases}

Horses are very sensitive to WEE infection, and are a useful sentinel animal for WEE virus activity. Diagnosis of WEE infection in horses is by serological confirmation of a rise in antibody titre, or a positive single serum sample (Neufeld and Nayar 1982). Onset of equine cases in most epidemics has usually occurred two weeks before human infections (Hess and Hayes 1967). In all but one of the recent outbreaks, the first horse cases preceded human infection by two $(1975,1977)$ or three (1981) weeks. In 1983 no horse cases were reported until after the declaration of a health emergency, and the initiation of an aerial spray program. A rate of new cases of six or more per week for two consecutive weeks is predictive of an outbreak (Neufeld and Nayar 1982).

An effective vaccine is available for horses. Not all horses are vaccinated, and some of those that are may still contract the disease. Young and therefore unvaccinated animals are particularly susceptible to virus infection. Use of equine cases as sentinels is compromised because the prevalence of vaccination of horses is not known. Only relative and indirect information can be gained from estimates of vaccine sales.

\section{Human Infection}

Monitoring of human cases is carried out to determine prevalence of WEE disease. Incidence of human cases is not an efficient predictive parameter. Human infections do not offer an early warning system. Infected humans show a variety of clinical symptoms which develop over at least eight to ten days, and often longer periods. Some cases go undetected until after the outbreak has been fully realized. Hence by the time human infections are diagnosed, the human population has already been at risk for, at least, eight to ten days, and perhaps considerably longer.

\section{Other Factors}

When indicators show departures from non-outbreak conditions, other factors tend to be considered in addition to defined criteria. These other factors include epidemiological features, previous experiences of disease outbreaks, and, following declaration of health emergencies, selection of target populations for control measures. In Manitoba and the bordering States, the Red River Valley area is considered to be at greater risk than western parts of the province, because historically, most indicators have occurred in the Red River Valley. The Red 
River Valley location has not incorporated into criteria for assessing risk, but was considered when assigning priority for aerial spray treatment during outbreaks.

Risk Assessment and Actionable Thresholds

Thresholds of risk are based on a combination of environmental and epidemiological conditions encountered during the season which are favourable to high levels of mosquito production, and high levels of virus activity.

Low Risk - Non-Outbreak Conditions

Temperatures normal or below normal levels; precipitation (snowfall and spring rainfall) at normal or below normal levels; Vector population abundance at normal or below normal levels ; No virus isolations from vector populations; Less than $4 \%$ rate of serconversion in sentinel flocks; No or occasional equine infections.

Action Response

Maintain routine monitoring.

Medium Risk

Above normal levels of up to four of the indicators. Greater level of risk if flock trap data or equine cases approach high risk level and with increasing number of indicators approaching high risk levels.

Consideration of all parameters is essential since any parameter in itself is not indicative of an outbreak. Mosquito populations can be high; but not support virus transmission (Reeves et al. 1961); and the virus can be present in the absence of high mosquito populations as is demonstrated by both horse cases and seroconversion in sentinel flocks in non-outbreak years.

\section{Action Response}

Increased frequency of trap collection, increased frequency of sentinel flock bleeding, reminders to veterinarians for closer scrutiny of possible horse infections, information exchange with neighbouring jurisdictions and local mosquito control authorities, close monitoring of weather reports, soil saturation levels, flood forecasting, preliminary advisory to senior health officials.

\section{High Risk - Epidemic Situation}

Mean weekly temperatures over the last two weeks of July and average mean weekly temperatures in July and August at least $2 \mathrm{C}$ above normal. Wet and warm April and May.

Trap collections of $75 \%$ or more Cx. tarsalis/flock

trap/week, and $35 \%$ or more of total trap catch of Cx tarsalis.

Fifty or more female Cx. tarsalis/ trap/week in NJLTs.

Virus activity levels resulting in $8.5 \%$ or more of $\mathrm{Cx}$.

tarsalis pools positive and a rate of $20 \%$ or greater seroconversion in sentinel flocks. 
Equine infections occurring at a rate of six new equine cases or more per week.

Occurrence of human cases is the primary criterion for implementation of control measures.

\section{Action - Advisory to Minister of Health}

Risk of outbreak was assessed on objective scientifically developed criteria. However, the same type of criteria are not available for determining both what remedial measures should be implemented, and where emergency (aerial) spraying should be carried out, if spraying is the chosen course of action. Selection of targets for emergency aerial spraying has been by size of population centre, despite the fact that WEE is primarily a rural disease, and if available, surveillance data. When aerial spraying has been adopted as a control strategy the procedure of systematically spraying all municipalities, priorized according to population size, has been used. It provides an objective device for selecting targets. In 1983, the U. S. Centre for Disease Control recommended to the Minnesota Arbovirus Surveillance Committee that ULV using malathion be implemented, and that the decision to spray areas should be made on the basis of population size and data on virus activity and infection. In the 1983 emergency program in Manitoba, some municipalities were sprayed which were both distant from surveillance sites and which had no earlier record of WEE activity.

More reasoned criteria for selecting sites for emergency aerial spraying are needed; lack of them are a deterrent to the implementation of a control option that can be carried out rapidly, on a wide-scale.

Criteria for control should also be developed to include cost-benefit of methods employed, i.e., compare the cost of impact of the disease the public, and costs of control procedures and surveillance against benefit of reducing human suffering and impact of disease and control measures on livestock and wildlife.

\section{b. St. Louis Encephalitis}

Following the only reported outbreak of St. Louis encephalitis reported for Canada, in Ontario in 1975, a special document was prepared by the Committee on Programs for the Prevention of Mosquito-borne Encephalitis (Mahdy et al. 1979), for the Ontario Ministry of Health. In that report, Mackenzie (1979) described, in detail, mosquito control efforts related to St. Louis encephalitis in Ontario. 


\section{CONTROL OPTIONS}

a. Vector Suppression

The mosquitoes carrying encephalitis virus from wildlife sources to people are the weakest link in the virus transmission cycle. Controlling the mosquito vector breaks that transmission chain and reduces the chances of people becoming infected with the virus. Thus, during epidemics of mosquito-borne encephalitis, every attempt possible is usually made to reduce the mosquito vectors that may be involved in virus transmission.

There are many reference works available on mosquito vector control. However, most of these guidelines, although very helpful, are not directly transferrable to the Canadian situation, having been written, in most part, for conditions in other countries (e.g. Anon. 1976, 1978, 1982, 1987, 1989; Monath 1980). Provincial mosquito control guidelines also may be very useful, particularly for starting the mosquito larviciding operations they usually emphasize (e.g. Brust et al. 1976). Also, reference can be made to an unpublished, 3-part technical manual on WEE surveillance and control in Manitoba (Ellis 1982a,b,c). In addition, a review of some of the papers describing the past emergency vector control programs in Manitoba and Ontario (e.g. Ellis 1976; Brust and Ellis 1976; MacKenzie 1979; Sekla 1982) would be useful.

Mosquito vectors can be controlled, under Canadian conditions, in both larval and adult stages. Generally, it is considered impractical to attempt to control mosquitoes in the egg stage and no insecticides are available in this country for pupal mosquito control. The only registered mosquito pupicide, Flit $M L O^{R}$, is no longer marketed in Canada. Thus, the choices are limited to mosquito larvicides and adulticides. Neither approach, mosquito larviciding or mosquito adulticiding is $100 \%$ effective. But, mosquito control can reduce the vector population and the chances of virus transmission.

Depending upon the timing of the encephalitis outbreak, one may carry out both mosquito larviciding and adulticiding or only mosquito adulticiding. When it is late in the mosquito season (i.e. after mid-August) and most, if not all, of the $\mathrm{Culex}$ larvae present will be giving rise to adult mosquitoes that will be entering diapause rather than blood-feeding, medical-veterinary entomologists usually will recommend that control efforts be concentrated on those mosquito adults that are already present and possibly infective. Such a mosquito adulticiding program is aimed solely at breaking the virus transmission cycle without concern about the next year's population. The decision would have to be based on a thorough assessment of the percentage of female mosquito vectors that had entered diapause.

Even late in the mosquito season, some workers might argue that both control options - mosquito larviciding and mosquito adulticiding - be taken because some of the newly-emerged mosquitoes may blood-feed, at least once, and, perhaps, carry the virus over the winter into the subsequent season. They might also 
argue that the public would perceive a new mass emergence of mosquitoes, even of a non-vector species, as a general failure of the vector control program and become sceptical of both the disease control efforts and the effectiveness of mosquito adulticiding.

If human cases were the only warning that a outbreak was in progress and those cases were not confirmed until late August, the best decision might be not to attempt any vector control. Without early indicators of virus and vector activity (i.e. an extensive surveillance program, including monitoring virus activity in sentinel flocks and mosquito vector population levels), an outbreak may be well advanced before it is recognized. In such a circumstance, personal protection (including repellents, screened doors and windows, wearing proper outer-wear, avoiding mosquitoinfested areas, etc.) might be the best (and only) course of action. Typically, there is sufficient time, once an imminent outbreak is recognized, to initiate vector control operations. Given even a basic vector and virus surveillance program, there may be a 2-4 week period during which vector control is possible. During this period and under optimum weather conditions, the vector population may increase while the virus amplifies in wildlife, resulting in many more human cases. Thus, the emergency mosquito control measures must not be delayed or their impact on the vector population will be greatly diminished.

\section{Non-chemical}

Ideally, mosquitoes could be controlled using non-chemical methods (Anon. 1965, 1977). Water management, in some jurisdictions, has been used successfully to maintain mosquito populations at acceptable levels. Saltmarshes, sewage and dairy lagoons, storm water-retention ponds, and even farm dug-outs can be managed to make them unsuitable for mosquito breeding through a combination of design, water level manipulation and vegetation control.

To a lesser extent, open ditches along road, rail and transmission line rights-of-way also can be managed to minimize mosquito breeding. Properly designed, so that they drain quickly rather than retain run-off water, and regularly maintained, so that sediments are removed and vegetation is kept low, such ditches do not have to be major sources of mosquitoes. However, good ditch management methods are very expensive compared to conventional mosquito larviciding, especially in the relatively flat land areas of the Canadian prairies.

Also, the public can be educated, over time, to recognize the importance of keeping private property free of water-filled containers. Leaf-plugged eavestroughs, discarded tires, buckets, bird baths, etc. that are full of stagnant water can contribute to neighbourhood mosquito problems. All of these various means of mosquito source reduction should be encouraged.

Biological control is another non-chemical alternative for mosquito control. The mosquito's natural enemies (including 
pathogens, parasites and predators) could be used more effectively against mosquitoes in their various life stages. Some successes have been reported in the southern U.S.A., especially the use of mosquito-eating fish. However, although mosquitoes have many natural enemies and these organisms undoubtedly have some impact on mosquito populations, they rarely maintain mosquitoes at acceptable levels. They have to be manipulated by man if they are to work effectively.

Only mosquito pathogens, particularly bacteria, have been successfully managed. Bacillus thuringiensis israelensis and Bacillus sphaericus have both been commercialized for larval mosquito control. Both bacteria can be mass-produced, packaged, shipped and stored for use. In the U.S.A., both bacteria are registered under several product brands. In Canada, only BTI is registered as a mosquito larvicide. The early larval instars are most susceptible to this bacteria. It has little effect on 4 thinstar larvae. Thus, it requires more understanding of mosquito biology to be used successfully. Although some argue that BTI is not truly a biological control agent because it is a complex chemical within the bacteria that actually kills the mosquito, BTI is a natural pathogen of mosquito larvae and it is certainly not a conventional chemical larvicide. Its use should be encouraged in on-going mosquito larviciding programs across Canada.

As much as these non-chemical means of mosquito control should be the basis of long-term mosquito abatement programs, they are of little value when one is faced with mosquito vector control after an outbreak of mosquito-borne encephalitis has been perceived.

\section{Use of Insecticides}

To deal with vector control during an outbreak of mosquitoborne encephalitis, there is currently no alternative to the use of conventional chemical insecticides. The only choices are which mosquito larvicides and/or adulticides to use, given the time, personnel and spray equipment available and the product label restrictions. The alternatives should be examined carefully, considering their cost, effectiveness and environmental acceptability.

Types and strategies

Mosquito control - whether it be larval or adult mosquito control - can be carried out using ground-based and/or aerial application equipment (Anon., 1974). From the ground, the sprayers may include manual or powered back-pack sprayers and truck- or trailer-mounted power sprayers. From the air, either rotary- or fixed-wing aircraft can be used to apply the insecticide. The insecticides may be applied as coarse or fine mists, as dusts or granules or as thermal or cold aerosols. Product choice depends on what spray equipment is available, whether larviciding or adulticiding is planned, and whether or not sufficient insecticide can be procured in the time available to meet emergency requirements. 
If an encephalitis outbreak is recognized early, mosquito larviciding may play an important role in preventing virus transmission. However, destroying the adult mosquito, a portion of which is carrying the virus from its first blood-meal, always must be the highest priority.

\section{Larviciding}

Mosquito larviciding is the introduction of biological or chemical insecticides into those aquatic habitats where mosquito larvae are believed to be present. Whether the larvicide is a biological material (e.g. the bacteria, BTI) or a chemical material (e.g. the organophosphate, Chlorpyriphos), the aim is to destroy the larvae before they emerge as adults and disperse throughout an area. It is much easier to destroy a million mosquito larvae in $1 \mathrm{~km}$ of roadside ditch than to destroy the resulting adult mosquitoes which may spread out over a 100 square $\mathrm{km}$ area. Also, it is usually more economical and environmentally acceptable to destroy mosquitoes in their immature stage.

If a decision is made to include mosquito larviciding in an emergency mosquito vector control program, it should be carefully planned and carried out. To have a significant impact on subsequent virus transmission, the larviciding program must be done as efficiently as possible. The spray equipment that is to be used must be already available or must be easily procured or hired.

Large truck-mounted sprayers should be used to treat major roadside larval breeding sites in and around urban and rural population centres. Rotary-or fixed-wing aircraft should be used to treat larger breeding sites (e.g. flooded woodlots, sloughs, wide drainage ditches). Helicopters are usually preferred over fixed-wing aircraft because of their greater manoeuvrability over smaller, off-road, breeding sides and their ability to land and be re-loaded quickly on farm access roads.

The ground and aerial larviciding programs must be coordinated and mobile. Ideally, the ground-based spray equipment and operators and the aerial spray crews will be under the supervision of an experienced mosquito abatement official with the authority and responsibility to larvicide in and around areas of highest priority based on larval surveys. The larviciding operation will, of necessity, have to be radio-equipped for good communications.

Both the ground and aerial operators would work in concert with each other, crews travelling from town to town as surveillance indicates the need for controlling vectors in the larval stage.

Also, "walk-in crews", equipped with motorized back-pack sprayers, could be used to treat small, off-road breeding sites that are either too small or too hazardous to treat using aircraft (e.g. small breeding sites located under transmission lines, between buildings or in backyards).

Such an emergency mosquito larviciding operation could be made to work efficiently, given proper supervision and authority. 
Its weakness is, of course, the likelihood that ground-based sprayers of the type used in mosquito control will not be available locally. Although helicopter spray crews and insecticide can be hired and procured fairly readily, or seconded from other provinces, it may take weeks or months to receive major spray equipment (e.g. Buffalo Turbine liquid-granular sprayer; John Bean mist-blower) ordered from U.S. manufacturers. Even a large, urban centre with an established mosquito abatement program rarely would have enough spray equipment to handle its own needs let alone be able to allow that equipment to be seconded.

Thus, an emergency mosquito larviciding program may be limited to an aerial application program, any ground-based equipment available restricted to the municipality fortunate enough to have it. Nevertheless, the aerial larviciding program, using helicopters and crews under contract, could make a significant contribution to the overall vector control program.

In an emergency situation, conventional chemical larvicides would be recommended. Biological larvicides (i.e. BTI) would not be acceptable because they are only effective, consistently, against 1st-3rd instar larvae. Most larval breeding sites containing populations of vector species would have a mixture of eggs, all larval instars, and pupae. Thus, one would choose an insecticide that would kill all larval instars and remain in the water for as long as possible, killing larvae that subsequently hatch. One insecticide having these qualities and formulated as both a liquid for ground-based sprayers and as a granule for helicopter application is chlorpyriphos. Others are listed in the Appendix.

\section{Adulticiding}

Mosquito adulticiding is the application of insecticides to destroy adult mosquitoes. It may involve "space spraying" which is the release of thermal or cold aerosol droplets into the air or "residual spraying" which is the application of a coarse liquid spray to vegetation where mosquitoes rest. Mosquito adulticiding (especially space spraying) has little effect on larval mosquitoes and they will continue to emerge as adults.

Space spraying may be accomplished with thermal or cold foggers, carried by hand, truck or aircraft. The small droplets that are generated by the sprayers drift through mosquito-infested areas, impinging on mosquitoes and killing them. After the droplets hit a surface and slowly evaporate or drift out of the target area, they no longer will kill mosquitoes. Thus, there is no residual carry-over of the insecticide. As a guide, as soon as the odour of the insecticide disappears from the area (usually within 1-2 hours), there is too little insecticide remaining to kill mosquitoes.

Space spraying is most efficient when done from the air (Akesson and Yates 1982). Ground-based fogging is obviously limited, except in large parks and golf courses, to areas immediately adjacent to roads, streets and lanes. The thermal or cold fog will drift downwind about $100 \mathrm{~m}$ before it begins to 
dissipate. Aerial ULV applications are not restricted in this manner, allowing large blocks to be treated more-or-less uniformly with mosquito adulticide. Aircraft are also much more efficient than truck-mounted foggers. For example, one DC-6 spray plane equates to about 150 truck-mounted foggers and the area coverage is about 10 times greater.

Many active ingredients are registered for use as space sprays for adult mosquito control. Some are limited to use with thermal aerosol generators (i.e. the old thermal foggers); others are registered for use in cold aerosol or ultralow volume sprayers (i.e. ULV or cold foggers). Some can only be used in groundbased equipment; others can be used in both ground and aerial spray equipment. Although there are several possible choices, only malathion and propoxur have been used in major emergency vector control programs in Canada. Both these products can be used from the ground or air and with either thermal or ULV sprayers. Each has its pros and cons but, at least, the problems with each are fairly well known and manageable.

Residual spraying is more limited in that only those mosquitoes resting in or attempting to rest in treated vegetation will be contacted and killed by the adulticide. Because residual sprays are usually limited to application using ground-based spray equipment, the total area sprayed is limited to vegetation reached from roadsides. Further, some vegetation (e.g. many sensitive flowers and shrubs) will be adversely affected by such residual sprays (unless water-based or very weak emulsions).

However, residual sprays can be used to advantage in roadside ditches, killing resting adult mosquitoes and, possibly, mosquito larvae breeding in flooded portions of those ditches. Such sprays may also destroy newly emerging adult mosquitoes and adult female mosquitoes returning to these sites to lay their eggs.

Many insecticides are registered in Canada as residual sprays for adult mosquito control. Those used most widely include chlorpyriphos and methoxychlor. Both would serve well during a health emergency, lasting 2-3 weeks on vegetation. Another alternative is Malathion. But, because of its short residual activity (lasting only a few days at best), it is seldom recommended for vector control programs where the duration of residual activity must be 10-20 days.

Comparison of Ground and Aerial Application

As noted above, both ground and aerial application have their place in both routine and emergency mosquito vector control programs, in both the larviciding and adulticiding operations. For large-area treatments, especially for adult mosquito control, aerial applications are generally more efficient and economical than ground-based applications. However, in the case of mosquito larviciding, many vector breeding sites are small or occur in narrow roadside ditches and they are dealt with most effectively using truck-mounted or back-pack sprayers.

Thus, assuming the resources were available, one would carry 
out mosquito larviciding from both the ground and air. If groundbased larviciding equipment is not readily available, an aerial larviciding program should still be considered. Although many small breeding sites might be missed, the aerial spraying might have a major impact on the mosquito vector population.

Large-area mosquito adulticiding is best carried out from the air during a health emergency. This mode of application provides for much quicker and more even coverage of an area with the adulticide at less cost than an incomplete, slower, groundbased operation. For example, under favourable conditions, a city the size of Winnipeg might be protected in one day using one large, multi-engine aircraft with an adequate insecticide load capacity. Under the same favourable conditions, it might take 10 days for 10 truck-mounted foggers to treat the same area, omitting portions that were inaccessible by road. However, it should be remembered that aerial applicators may not necessarily be available during a health emergency. If this were true, even a slow, incomplete, ground-based, adulticiding operation would be preferable to nothing.

In an ideal situation, one in which both ground and aerial application equipment is available, both types of equipment could be used to advantage in an emergency situation. The ground-based equipment may be mobilized faster and carry out mosquito adulticiding while the aircraft is being readied. Further, the truck-mounted equipment might be used, after the aerial application has been done and moved on to other areas, to "touch up" areas where either new adult mosquito emergences have occurred or areas where the level of control from the aerial application was unacceptable. Truck-mounted equipment could also be used to treat a series of small towns while the aircraft was used to treat larger centres.

\section{Control Requirements/Procedures}

Emergency vector control requires trained personnel, spray equipment and insecticide(s). Ideally, each province which has had a history of outbreaks of mosquito-borne encephalitis will maintain an up-to-date inventory of the resources available to deal with possible future outbreaks. Knowing who and what is or can be made available is crucial to a rapid response to such an outbreak.

Fortunately, most such provinces have ongoing mosquito abatement programs in some of their larger cities or regions. The staff involved in these programs can advise on what manpower and equipment they may have available to assist. From this base of experienced mosquito control officials, the province may be able to appoint someone to direct the emergency spray operations. In addition, these officials can suggest suppliers of needed insecticides either from within the province or nationally, through local sales offices, based on recent calls for insecticide tenders that they have made.

Following their advice could save the province considerable money in purchasing insecticides. These people can give an 
objective assessment of how the available insecticides perform locally and on the past service records of suppliers. These mosquito abatement agencies may also be able to supply needed insecticides, on a replacement basis, before the commercial suppliers can produce and deliver materials, enabling an emergency program to begin operation as early as possible. Similarly, major mosquito abatement agencies in adjacent provinces may be contacted for advice, and possibly emergency start-up supplies of materials.

Two major considerations in large-area mosquito vector control programs are the insecticide to use and the contractor to hire for its aerial application. The insecticides currently registered in Canada for mosquito control, using aerial ULV application equipment, are propoxur and malathion. Both have environmental pros and cons but both have been used successfully in Canada for emergency vector control. Their most current unit costs and costs of application can be compared when an emergency occurs in order to determine which oe to use.

When choosing an experienced aerial applicator with multiengine aircraft, equipped with the specialized ULV spray nozzles required for vector control, the choices are more limited. Only one aerial application firm in Canada has experience with largearea, emergency mosquito vector control. Conair Aviation of Abbottsford, B.C., was the aerial applicator that carried out the emergency spraying in Ontario in 1975 and in Manitoba in 1975, 1977,1981 , and 1983.

One should expect local aerial application firms, usually small businesses with 1 or 2 ag-planes, each equipped with conventional boom and nozzle sprayers, to lobby for the aerial application contract. Local industry should be supported, all things being equal. However, it must be emphasized that having the proper equipment and experienced staff is most important.

One DC-6, with 2 crew members and having a 16,000 L insecticide capacity and equipped with the specialized ULV nozzles needed for its application, can treat 44,500 hectares in under 4 hours. For small ag-planes to do the same work in the same time, one would need 69 ag-planes, 46 spotter planes, and a fleet of insecticide and fuel supply trucks. One should also consider that 69 aerial applicator agreements would have to be developed.

The most important consideration in selecting an aerial applicator is safety in the air. Perhaps, the best advice such lobbyists can be given is for them to acquire equipment and gain experience with non-emergency mosquito control. Some of them would then be in a position to assist in future vector control programs.

Every effort should be made to contract with the suppliers of insecticide and aerial application services as soon as the need for emergency vector control is recognized. Even after these suppliers are designated, there will be many problems to solve.

\section{Relevant Provincial and Federal Legislation}

Many federal and provincial Acts and regulations relate, directly or indirectly, to routine and emergency mosquito vector 
control operations. The federal Pest Control Products Act and associated regulations are, of course, the key concerns.

Basically, the Act states that only registered insecticides may be used and then only in accordance with approved label directions and precautions. This should not present any problem in vector control programs but should certainly be followed. If any ambiguities are perceived, advice should be sought from the Pesticides Directorate of Agriculture Canada and followed.

If migratory bird refuges are contained within proposed spray blocks, attention should be paid to the Canada Migratory Birds Convention Act, and close coordination maintained with local wildlife authorities. Generally, such refuges should not be included in emergency spray programs unless the risk to human health outweighs any possible risks to waterfowl and their habitat.

Most provinces also have Acts and regulations governing the use of pesticides (e.g. Alberta Agricultural Chemicals Act, Manitoba Pesticides and Fertilizers Control Act, Manitoba Environment Act, Ontario Pesticides Act). Also, the provinces may each have several other Acts that indirectly affect pesticide applications (e.g. Environmental Assessment Acts, Workplace Safety and Health Acts, Transportation of Dangerous Goods Acts, etc.).

Provincial regulatory officials should be consulted early to ensure that all applicable regulations under these Acts are either followed during routine mosquito control operations or, if necessary, waived for the duration of emergency vector control operations.

\section{Aerial Insecticide Application}

Mosquito vector populations can be reduced fairly quickly by emergency wide-area aerial applications of insecticide. After the human population centres that are at risk from mosquito-borne encephalitis are identified, plans can be made to aerially spray these centres and an adequate area surrounding each of them. The centres may be identified by the occurrence of human cases, horse cases, sentinel flock virus activity and/or high vector

populations. These centres may be priorized on the basis of human population levels and/or incidences of virus activity.

Once known, detailed maps (usually the most current topographical maps that are available) for these cities and towns can be obtained and the spray blocks drawn onto them. Usually, there is only one spray block per small city or town and 3 or 4 spray blocks per larger city. The spray block is centred over the population centre and includes a buffer zone of up to $10 \mathrm{~km}$ around the town or city.

The topographical maps usually show the location of obstacles to low-level flight, including radio and television transmission towers, water towers, etc. Flight lines can be drawn to avoid these obstacles. The longitude-latitude coordinates given on the map can be used to set-up the computerized flight navigation equipment, ensuring precise start and finish points for each of the parallel flight lines within the spray block. 
When the number of populated centres is known and the spray block maps have been developed, the total surface area to be treated will be known (although additional centres might be added after the spray operations begin). Once the treatment area is known, the amount of insecticide required can be calculated and ordered. Also, based on the number of spray blocks and their size, negotiations can begin with the contractor on aerial application costs.

Typically, there are fees associated with moving aircraft into the province, daily positioning fees at the airport, maintenance fees for holding the aircraft while awaiting acceptable weather conditions for spraying, and fees for the actual spraying. A fairly accurate picture of the costs associated with the health emergency can be developed once the spray blocks are identified, the insecticide is ordered and the contract with the aerial applicator has been finalized.

While all of this is happening, the emergency spray operations base can be set-up at the closest international airport, the public information centre can be organized, groundsupport personnel can be arranged, legal matters can be attended to, surveillance can be accelerated, spray monitoring and evaluating procedures developed, reporting procedures agreed to, etc.

One should expect that local environmental groups and individuals will attempt to stop the emergency spray operations. Reasons may vary but usually will centre on health and environmental concerns. Tactics may include media reports on the real and/or imagined effects of similar, past spray programs, attempted court injunctions based on harmful effects of spraying on the health of individuals or groups or the government's ignoring its own Acts and regulations. Everyone involved in the health emergency can expect to be affected by the challenges these tactics present.

Matters may become somewhat chaotic as all the various agencies are trying to either organize themselves, ensure various federal and provincial regulations are being followed, fight legal battles, etc. Events are often further complicated because key decision makers are on vacation during late July and early August. However, once the usual start-up problems are overcome, the spray operations usually settle down to the business at hand.

\section{Weather Conditions}

The success of aerial ULV insecticide applications is closely tied to the weather conditions that occur during and immediately after spraying. Thus, meteorological data are needed for operational purposes throughout the duration of the emergency spray operations. These data not only determine when and where spraying will take place but also may be important in the monitoring of spray dispersal, the evaluation of vector mortality, the assessment of disease risk and the investigation of damage claims. Obviously, the full cooperation of the Atmospheric Environment Service is required. 
Ideally, the aerial applications will be carried out when weather conditions and time of day favour both uniform insecticide droplet dispersal and mosquito flight activity. Realistically, weather conditions over a spray block must sometimes be extrapolated from nearby observation points and, because a spray flight may take 3 hours to complete, changes in weather conditions over the spray block can occur during the spray operation. Whereas the person directing spray operations may make the decision to begin spraying, in consultation with meteorologists and the spray crew supervisor, the ultimate decision is made by the pilot. The pilot is responsible for the safe operation of his aircraft and may decide, on the ground, that the flight cannot take place for mechanical or other reasons or, in the air, that the flight be aborted for weather or mechanical reasons.

Spraying should occur if the following weather criteria are met: i.e. temperatures of $12-28^{\circ} \mathrm{C}$, relative humidities of 35 $95 \%$, wind velocities of 4 to $16 \mathrm{~km} / \mathrm{h}$, wind direction at an angle to the flight lines (the closer to perpendicular the better), and adequate light for visual flight. These criteria for spraying are difficult to meet. Each factor must be considered in relation to the others and a judgment call must be made. Frequently, the decision to begin spraying on a given evening or morning will be made at the last minute to take advantage of favourable weather conditions at any one of several possible spray zones.

This last-minute decision-making, although unavoidable, often causes problems for personnel involved in informing officials and the public and monitoring the spray operation, underlining the need for good communications equipment and procedures.

Environmentally-sensitive areas

Environmentally-sensitive areas, over which direct spraying during a health emergency should be avoided, if at all possible, include those areas where there are large gatherings of people, outdoors during the actual spraying, and those areas which are truly environmentally-sensitive. Examples of the former include such recreational events as major football games in open stadiums and large, special weekend events in major parks. Examples of the latter include bird and other wildlife refuges and zoos where large assemblages of rare or endangered wildlife may be present.

Adjustments to the spray block design must be made to account for some of the environmentally-sensitive areas if these occur on the periphery of the spray block. Otherwise, the spray can be turned off just before the aircraft flies over the area involved.

Requests may be received to omit small areas for one reason or another (e.g., a hospital complex, a golf tournament) but it is impractical to turn the spray off for these areas. In the case of hospitals, food processing complexes, etc., maintenance personnel can be advised to close windows and turn off air conditioning equipment for a few hours. Small, recreational events can be postponed or cancelled if the organizers are concerned. 
Routine mosquito control operations may also affect environmentally sensitive areas. The same precautions should be followed. Because routine operations involve smaller areas, at least on a day-to-day basis, many small but sensitive areas can be excluded from spraying, including buffer areas adjacent to persons registering their opposition to spraying.

\section{iv. Activity Periods of Vectors}

An adult mosquito vector control program must consider mosquito behaviour patterns. Host-seeking and dispersal flight in Cx. tarsalis peak between 30 minutes after sunset and midnight and at about 30 minutes before sunrise (Bailey et al. 1965; Nelson and Spadoni 1972). Adults leave daytime resting sites when the light intensity decreases to $<30$ lux and seek daytime resting sites shortly before sunrise, when light intensities increase to > 80 lux (Gjullin et al. 1963). A variety of natural habitats (e.g. tall vegetation, wood lots, animal burrows) and artificial habitats (e.g. culverts, chicken houses and other farm buildings) serve as daytime resting sites (Mitchell et al. 1980).

For maximum vector suppression, mosquito adulticiding should be conducted during the peak flight and host-seeking periods of the day. The most effective periods for adulticiding would be after sunset and prior to sunrise. Aerial adulticiding cannot be conducted during those times, as aviation regulations in Canada restrict low level flights over urban areas to daylight hours. Therefore aerial applications of ULV insecticides for vector control in urban areas have been made in the early evening hours, when temperatures are above 10 to $12 \mathrm{C}$ and when wind speeds are below $16 \mathrm{~km} / \mathrm{h}$ (Ellis 1976). Ground-based adulticiding, with ULV aerosol sprayers, is usually conducted from $2100 \mathrm{~h}$ to $0500 \mathrm{~h}$, the period of greatest flight activity in host-seeking mosquitoes (Ellis 1976).

When control of $C x$. tarsalis is carried out to reduce the risk of human infection of WEE virus, it is important to determine when blood-feeding activicy ceases and when populations prepare for overwintering. Because females overwinter as non-blood fed, nulliparous individuals (Blackmore and Dow 1962; Bennington et al. 1958b; Burdick and Kardos 1963), with sufficient lipid reserves to last until spring (Kliewer et al. 1969; Schaefer and Miura 1972; Schaefer et al. 1971; Schaefer and Washino 1969, 1970), such females would not seek a blood-meal and would not be involved in virus transmission.

Methods for assessing when diapause begins in $C \mathbf{x}$. tarsalis populations are not well defined (Buth 1983). Measurements of the relative size of ovarian follicles and the germarium in females is one method of evaluation. When serial populations of a Manitoba strain of $C_{x}$. tarsalis were reared outdoors in Winnipeg, and adults maintained outdoors for 2 weeks, some females were in diapause by $27 \mathrm{July}$. By 9 August, 70 to $75 \%$ of the females were in diapause (Buth 1983). Another indicator of diapause may be the rapid reduction in females coming to chicken shed traps and $\mathrm{CO}_{2}$ traps while egg rafts and immatures of $C x$. tarsalis are abundant, 
and nightly temperatures are considerably above the threshold for flight. The precipitous drop in numbers attracted to chickens during August 1981 (Brust 1982) and to $\mathrm{CO}_{2}$ traps near Winnipeg during August 1985 and 1986 (Brust unpublished) would suggest that diapausing females of $C x$. tarsalis are not being trapped. Similar reductions in $C x$. tarsalis occur in light trap collections in Winnipeg during August (Raddatz 1985).

Light traps captured as many parous $C x$. restuans and $C x$. salinarius as $\mathrm{CO}_{2}$ supplemented 1 ight traps (Magnarelii 1975 ; Feldlaufer and Crans 1979) indicating that light traps may be sampling mosquitoes in the same physiological state as those attracted to $\mathrm{CO}_{2}$ traps. If this is so for $C x$. tarsalis, it might explain the rapid drop in light trap catches at Winnipeg in early August 1979, at a time when weather conditions suggested trap collections should be increasing (Raddatz 1985).

The threshold light trap index of $C x$. tarsalis necessary to transmit WEE virus to humans has not been determined for Manitoba. If isolations of the virus in $C x$. tarsalis are made in mid-summer and the seroconversion rate in sentinel chickens exceeds $20 \%$, an epizootic and an epidemic of WEE may occur (Wong and Neufeld 1982). Before vector control is initiated on an emergency basis, assessments of parity and diapause rates in $C x$. tarsalis should be determined. Several collection methods should be used to sample the vector population, and properly evaluate the risk of infection to humans.

v. Honey Bee Activity

An extensive review of the effects of pesticides on bee pollinators may be found in Johansen (1977). Practical aspects that should be considered in mosquito control operations involving agricultural areas, and by beekeepers who may be affected by an insecticide application, are given by Atkins (1975) and McGregor (1976). The main factors are 1) honey bees may be foraging on flowering plants during all hours of the day, but primarily when temperatures are above $20 \mathrm{C} \mathrm{2)} \mathrm{honey} \mathrm{bees} \mathrm{may} \mathrm{be} \mathrm{killed} \mathrm{by} \mathrm{direct}$ contact with insecticide droplets during flight or while gathering nectar or pollen 3 ) the insecticide may be ingested with nectar or pollen, and kill as a stomach poison 4) bees may die in the field if the insecticide acts quickly, and bee death may not be observed by the beekeeper. With slow acting insecticides bees may return to the hive where they may die at the entrance or inside the hive 5) bees may forage $2 \mathrm{~km}$ or more from the hive, and beekeepers may suffer losses even if their hives are outside the target area 6) insecticide drift, especially from ULV applications may carry beyond the target area 7) the insecticide used should be selectively toxic to mosquitoes and should degrade as quickly as possible after mosquitoes are killed.

When mosquito adulticiding operations are conducted in agricultural areas, honey bees foraging for nectar or pollen are at risk. Aerial ULV applications of propoxur and malathion (during 1981 and 1983, respectively) in Manitoba killed significant numbers of honey bees in all the treated areas of the 
Province (Dixon and Fingler 1982, 1984). Direct losses to beekeepers in 1981, when propoxur was used, totalled $\$ 87,455$. Honey bee and leafcutter bee losses in 1983, when malathion was used, totalled $\$ 846,429$. Beekeepers and leafcutter bee operators were compensated by the Province of Manitoba for the loss of bees, and the estimated losses of honey and alfalfa seed production that resulted from the emergency vector control operation (Dixon and Fingler 1982, 1984). The greater losses in 1983 were due to the more extensive agricultural area within the treatment zone and the greater toxicity to bees of malathion compared to propoxur (Dixon and Fingler 1984). 
b. Host Protection

Most female mosquitoes require a blood-meal in order to develop their eggs. The source of this blood is called their host. Preventing mosquitoes from biting an individual person or animal is termed host protection. Some people will argue that the best way to protect people and domestic animals from mosquito bites and mosquito-borne disease is host protection. Others will argue that host protection, although important, is only one component of protection from mosquito-caused annoyance and disease.

Regardless, in certain situations, host protection may be the only means of relief from mosquitoes. Thus, this section attempts to describe the various alternatives that are available. Discussion is limited to the protection of humans and horses, the two hosts most likely to be seriously affected by mosquito-borne encephalitis viruses.

\section{Suitable Clothing}

People who must be outdoors while mosquitoes are active can minimize mosquito bites by wearing appropriate clothing. Loosefitting, long-sleeved shirts, slacks, socks and hats reduce the skin surface area that is exposed. If this clothing is made of a thick, tightly-woven material, mosquito bites will be minimized.

Because mosquitoes are more attracted to dark colours it helps to wear light-coloured clothing. Orange, yellow, beige, and pastel colours reportedly are among the least attractive colours. On the other hand, purple, navy-blue, brown, and other dark colours are very attractive to mosquitoes.

Unfortunately, temperatures are often high during July and August when encephalitis outbreaks occur. People tend to wear shorts and t-shirts rather than the heavier clothing noted above. Sometimes, the use of such summer-wear may be balanced by applying repellents to this greater area of exposed skin.

Some new light-weight, light-coloured, tightly-woven fabrics are being developed for people who spend considerable time outdoors. Marketed as "sun-screen" clothing, pants and jackets made of these materials may afford some protection from mosquito bites. Because of their tight weave, some of these synthetic materials do not breathe very well, causing a build-up of moisture inside and subsequent discomfort. However, for relatively inactive people, sitting outdoors, they may prove to be an alternative means of protection.

\section{i1. Screening and Netting}

People can reduce their exposure to mosquitoes by using screens to prevent mosquitoes from entering their buildings, homes, mobile homes, tents, etc. Windows, doors, vents and other openings should be covered with mosquito-proof screening.

Screens ( $16 \times 16$ or $14 \times 18$ meshes to the inch) to prevent mosquito penetration must be well maintained. Screens with holes or tears should be repaired or replaced before the mosquito season 
begins. If a hole is present, some mosquitoes will find it. Because mosquitoes often will attempt to follow people indoors, screen doors should have fast-acting, automatic closing devices. Perhaps, the simplest such device is the old-fashioned spring-return.

Infants in indoor cribs or outdoor carriages can be further protected by mosquito netting. A rectangular piece of thin, cotton or nylon material (23-26 mesh per inch) can be fastened over the crib or carriage to prevent mosquitoes from biting resting infants. Similar "bed nets" or "mosquito bars" could be made for campers resting in tents that do not have proper screening.

Head nets may be used by older children and adults who may be sensitive to the use of repellents. Unfortunately, head nets are usually uncomfortable and awkward to wear, and consequently, are used only when mosquito annoyance levels are unbearable. The most comfortable are those that use a dark coloured netting, with 14-16 meshes per inch. Often sold as bee veils for use by beekeepers, these devices are an effective means of preventing bites on the head area.

Air curtains can be used to prevent mosquitoes from entering loading and access doors (e.g., in commercial storage and maintenance facilities) where screening may be impractical. Ultraviolet insect electrocution traps, placed indoors, may be used to kill mosquitoes that are able to enter such buildings.

Screened gazebos and porches are another means of minimizing mosquito bites while enabling people to enjoy their evenings outdoors.

Related to this matter, some workers advocate applying an approved, residual insecticide to screen doors and windows and surrounding surfaces. Screens can be treated by using a paint brush to apply the diluted insecticide.

\section{Behaviour Modification}

Because most mosquito vectors are most active during the periods of dawn and dusk, avoidance of human activity during these periods, especially in areas of high mosquito infestation, would reduce the frequency of human-mosquito encounters, and thus, the risks of disease transmission.

Providing this basic information to the public, before and during an encephalitis outbreak, and encouraging them to schedule their recreational activities accordingly may afford some protection. Unfortunately, many people will either ignore this means of personal protection or are not in a position, for occupational (e.g., farmers harvesting their crops) or other reasons, to follow these simple precautions.

During an outbreak of encephalitis, public health officials should make every possible effort to motivate people, particularly those individuals who are in high risk categories (e.g., infants; farmers), to avoid exposure to mosquitoes. Suggesting that activities be carried out in open, sunny, windswept areas during the middle of the day rather than in heavily-vegetated, shady, 
protected areas during the evening may reduce the risk of mosquito bites and possible disease transmission while recognizing the need for people to be outdoors during the mosquito season.

iv. Topically-applied Repellents

If mosquitoes cannot be avoided, one method of reducing mosquito bites is to repel them. Insect repellents applied to exposed skin disrupt the attacking mosquito's sensory organs, essentially confusing them and preventing them from biting.

Repellents can be purchased in a variety of forms including liquids in squeeze bottles, pressurized spray cans, sticks, foams and wipes. They may be scented or unscented. Others may be formulated as combination repellents-sun screens. Also, many different active ingredients, alone or in combination, are used in repellents.

The active ingredients most commonly found in repellents include the following:

- Oil of citronella;

- Citronyl;

o N,n-diethyl-m-toluamide (DEET);

- Dimethyl phthalate;

o Ethylhexanediol;

- Oil of lavender;

- Bisbutylene tetrahydrofurfural (MGK R-11);

- Di-n-propyl isocinchomeronate (MGK 326);

- N-octyl bicycloheptene dicarboximide (MGK 264).

DEET, alone or in combination with one or more of the other active ingredients, is the primary ingredient most commonly found in repellents. The per cent active ingredient present in a formulation may vary considerably from product to product. Given the bewildering number of choices available, people may be confused as to which repellent to buy (Appendix 1).

Some people choose to make and use their own mosquito repellent. Home-made preparations and remedies include animal grease, vitamin B12, lemon peels, oil of citronella, baby oil, bath oil, etc. Although some of these materials may provide some protection against mosquitoes, commercial repellents generally are more dependable. Those repellents having DEET as the primary active ingredient will provide an acceptable level of protection against mosquito bites.

When repellents are applied to exposed skin, they usually can prevent mosquito bites for 2 hours or more, depending on the mosquito species involved, the numbers of mosquitoes present, the formulation used and the user's level of activity. Repellents are removed from the skin by absorption, evaporation, rain, sweating, swimming or wiping. Usually, repellents must be reapplied every few hours to maintain their effectiveness.

Some repellents, in aerosol form, can also be sprayed onto clothing (e.g., socks, cuffs) to enhance their protection. Repellent applied to clothing usually will last longer than when 
applied to skin. If people conscientiously use repellents while they are outdoors, in combination with other personal protection measures (e.g., suitable clothing), the risk of being bitten by mosquitoes will be reduced significantly.

Repellents should, of course, be used carefully, following label directions. Many repellents are solvents, damaging paints, lacquers, varnishes, nail polish, and other plastic materials. Susceptible plastic materials may include watch crystals, eyeglass frames, golf clubs, helmets, telephones, furniture, seat covers, pens, and certain dynel-or rayon-based fabrics.

Because personal repellents are applied to the skin, their safe use is very important. They should not, as a rule, be applied to infants or toddlers. An adult should apply them to young children, taking special care to avoid contact with the eyes, lips and nose. Older children should be instructed in the safe use of repellents.

Aerosol repellents are best sprayed into the open hand and then rubbed onto the face and neck rather than spraying directly. They should not be sprayed near the mouth or eyes of humans or pets.

Although very uncommon, some people may have an adverse reaction to a repellent. This may range from a short-term, allergic skin reaction to a toxic reaction such as generalized seizures. Ingestion of DEET may be fatal. Persons who are sensitive to known repellents should be especially careful. Excessive or prolonged use of a repellent, particularly by young children, should be avoided.

If a slight allergic reaction to one repellent is experienced, switching to a repellent with a different active ingredient (e.g., from DEET to oil of citronella) may correct the problem. In case of a serious reaction, one should seek medical attention immediately.

\section{v. Repellent-impregnated clothing}

Many people want to be or must be outdoors for extended periods of time while mosquitoes are active. As an alcernative to the repeated, topical application of a repellent, some people have found repellent-impregnated jackets (usually hooded and with optional matching pants) to be an effective means of personal protection. The active ingredient used to impregnate the fabric is usually DEET or citronyl. Some manufacturers claim that such jackets will provide up to 32 hours of protection per charge.

Typically, a light-weight, light-coloured mesh jacket is impregnated with repellent by placing it into a storage bag and adding 10-25 mL of repellent. After several hours, the jacket may be used. Most such jackets can be re-charged with repellent and used over and over. These jackets are popular among fishermen, campers, hunters, linemen, surveyors, farmers, photographers, lumberjacks, roughnecks, boaters, gardeners, golfers, and other outdoor workers.

The repellent-impregnated jackets do have some problems. They are not very durable, especially when worn while walking or 
hiking through wooded areas, often catching on branches and snagging or tearing. They also pose a risk around fires because of their flammability. They should not be worn near open flames or while smoking.

\section{vi. Personal Hygiene}

Mosquitoes may be attracted to people by the carbon dioxide they exhale, their movement, the colour of their clothing, their body heat and their body odours. Although there is little scientific information available on this topic, persons who perspire heavily, work under strenuous conditions, and thereby generate significant body odour may be more susceptible to mosquito bites, other things being equal. Thus, good personal hygiene, coupled with the other protective measures, may reduce the number of mosquito bites that person would otherwise receive.

For the same reason, people may wish to avoid the over-use of colognes and hair sprays, some of which may be attractive to mosquitoes.

\section{vii. Human Vaccination}

Fox (1982) assessed the use of vaccination to protect humans from WEE. Currently available only to a relatively few laboratory and field workers who are at greater risk, human vaccination, if feasible, would be a means of controlling outbreaks of encephalitis in endemic areas. Although some research has been carried out on human vaccines, none of the vaccines developed have proven to be sufficiently antigenic to produce long-term immunity.

Because of the high research and development costs that would be involved, the risks and costs associated with largescale vaccination programs, the instability of developmental vaccines and the infrequent occurrence of encephalitis outbreaks, it would appear that human vaccination for protection against mosquito-borne encephalitis currently is not a practical alternative.

\section{viil. Devices for Protection}

Many gadgets are marketed, from time to time, claiming to protect people from mosquito bites while they are outdoors. These include small, battery-operated mosquito repellers and backyard zappers.

A11 of the electronic devices that have been subjected to scientific investigation have proven not to have any significant effect on the number of mosquito bites that a person will receive.

\section{ix. Horse Vaccination}

Horses are especially susceptible to mosquito bites because of the potential for prolonged exposure in the field. Many horses have, over the years, been bitten by mosquitoes carrying encephalitis virus. Some suffer nervous system disorders; others, death.

Since the late 1930 's, a vaccine for protecting horses against WEE has been available. A vaccine has also been developed 
that protects horses against both WEE and EEE in those areas where both diseases are endemic.

Vaccination programs have led to a significant reduction in the number of horse cases of WEE in Manitoba over the past 15 years. Theoretically, it is possible to protect all horses against these diseases (i.e., WEE, EEE) in Canada. However, because horses are dead-end hosts and, thus, play no role in the spread of these diseases to humans, vaccination of horses will not protect humans (Anonymous 1978).

Horse owners wishing to protect their stock should consult their veterinarian for advice on this matter.

\section{x. Repellents for Horses}

Many repellents are available for use on horses to protect them from mosquito bites (Appendix 1). These repellents may be applied by spray or cloth pad. Their main drawbacks include their short duration of protection (usually less than 3 hours), their cost and the time it takes to apply them to one or more large animals. Most veterinarians and farm supply stores carry a range of approved repellent formulations.

\section{xi. Smudges}

Smudges, are an old-fashioned, yet effective, method of repelling mosquitoes within a limited area. Common in rural areas and camp-sites (but usually not allowed by law in urban settings), smudges have been used to protect people and livestock from the bites of mosquitoes and other biting flies.

Smudges often are made by placing rotting wood, leaves or damp straw over a well-established fire to generate clouds of smoke. Horses quickly learn to stand downwind in the smoke to obtain relief from mosquito bites.

\section{xi1. Insecticides}

There are several inseticides registered in Canada for protection of horses from mosquito attack. Because of local problems with availability of various insecticide formulations, persons interested in using these materials are advised to consult their regional agriculture extension representative. 


\section{EMERGENCY VECTOR CONTROL}

a. Emergency Vector Control by Municipalities

As outlined above, in the section on vector suppression, emergency vector control (whether it be a large-scale provincially-operated program or a smaller-scale, municipallyoperated program) is much more than simply deciding to spray, ordering some insecticide and hiring staff and equipment to treat the community(ies) at risk. The many elements of such an operation must be carefully considered before beginning an emergency spray program. In this section emergency mosquito vector control by municipalities, either alone or in conjunction with a provincially-operated vector control program is discussed in general terms.

Most, if not all, emergency vector control programs carried out by municipalities will be done in conjunction with a provincial program. It would be difficult to conceive of a city or town attempting to initiate an emergency program without prior provincial approval, financial support or other involvement. Any municipal mosquito vector control program, whether routine or emergency, would require approval from provincial regulatory agencies. Further, it would be relatively easy for a province to stop a municipality from carrying out an emergency program with which it did not agree, simply by revoking their pesticide applicator licences and program permits. Thus, if an emergency situation developed in only one municipality, nothing of an emergency nature could be done without that municipality and the province agreeing on a course of action.

The reverse would not necessarily hold. If a municipality did not wish to be included in a provincially-operated vector control program, it would have little power to stop the operation. Obviously, any political differences which might exist between the two governments would have to be put aside during a localized or regional mosquito-borne disease outbreak and they would have to cooperate fully with each other.

Based on Manitoba's experience with WEE outbreaks, a municipality with an on-going mosquito control program would be in a good position to step-up its operations from routine to emergency vector control almost immediately. Following political approval of additional expenditures for the accelerated vector control program, the necessary extra personnel could be hired, materials could be purchased, and the program could be expanded. Such local efforts should be encouraged because they could offer a degree of protection to the public while the province was involved in organizing a larger-scale vector control program.

Initially, the municipal vector control operations might be done independently but, once the provincial program started, the 2 programs would have to be coordinated.

If the municipality had the necessary resources, both mosquito larviciding and adulticiding could be carried out. Given the required trained staff, spray equipment and insecticides, mosquito larviciding could be carried out during the day and 
mosquito adulticiding during the night. A municipality with limited resources might concentrate its larviciding efforts on treating known vector breeding sites (for Culex tarsalis, these sites would include discarded tires, barrels, and other containers, plus such temporary and semi-permanent aquatic habitats as ditches, sloughs and marshes) and its adulticiding efforts on applying residual sprays to areas adjacent to these breeding sites.

On the other hand, a municipality with adequate resources might expand its normal larviciding zone around its built-up areas, reducing inward mosquito dispersal, and might adulticide all of its residential areas on a rotational basis, killing adult mosquito vectors already present throughout the city or town.

The key elements of such municipal mosquito vector control programs are described below.

Vector Control Methods, Materials and Equipment

Most on-going mosquito abatement programs emphasize mosquito larviciding. Larvicides are applied to known mosquito breeding sites to destroy the immature mosquitoes before they become a nuisance or health hazard. Mosquito adulticiding, on the other hand, is usually considered a back-up measure, designed to reduce adult mosquito populations, if and when larviciding has been inadequate for some reason.

In the case of new mosquito larviciding programs, most of the larval breeding sites are mapped out before spraying begins. Over the course of several years, abatement officials develop an inventory of such breeding sites, enabling more efficient larviciding operations to be carried out. A municipality that has had such a program for many years is in an excellent position to carry out an emergency larviciding program.

\section{Mosquito Adulticiding}

If a municipality does not know where the mosquito breeding sites are when a health emergency is perceived, it might be best advised to forego any attempt at larviciding, concentrating all of its resources on mosquito adulticiding. The main target, in any case, would be the adult mosquito vector. Only the adult female mosquitoes may have the virus after they have become infected from their first blood-feeding and are then capable of transmitting the disease. The only way in which these infective mosquitoes can be destroyed efficiently is by a mosquito adulticiding operation.

Mosquito adulticiding involves either the application of thermal or cold insecticide aerosols along street and lanes (thermal fogging and ultralow volume [ULV] spraying or cold fogging, respectively) or the application of residual sprays to mosquito resting areas. The adulticide may be applied by backpack sprayers or by truck-, trailer-, or aircraft-mounted sprayers.

Most municipalities with established mosquito control programs will have ground-based programs. Some may supplement their ground-based larviciding operations with aerial larviciding 
(e.g. Winnipeg), mainly using helicopters. A very few may have the capacity to carry out both aerial larviciding and aerial adulticiding (e.g., Edmonton). Such well-equipped operations will be directed by experienced professionals who have acquired expertise over a period of years. Aerial adulticiding is described in other sections of this manual. The discussion below will be limited to describing ground-based, emergency, mosquito adulticiding programs which can be implemented fairly quickly, given the necessary resources.

It should be emphasized that mosquito adulticiding will only reduce mosquito vector populations temporarily. Even if $95+\%$ of the mosquitoes present are destroyed, the remaining mosquitoes still have the potential to transmit the virus. Over the course of several days, additional adult mosquitoes will add to this residual population, dispersing into a treated area from neighbouring untreated areas and emerging from larval breeding sites within the treated area. Thus, the public should not rely entirely on the municipality's efforts to control the mosquito vector. Although the vector population may be decimated by mosquito adulticiding operations, the public still should be encouraged to use personal protection to further reduce the risk of being bitten by any remaining infective mosquitoes.

\section{Fogging}

Generally, fogging is more effective, efficient, economical and practical than residual spraying. The "fog" (i.e. minute aerosol droplets) generated by the specialized sprayers that are used in mosquito fogging is carried about $100 \mathrm{~m}$ downwind through an infested area, killing resting and flying mosquitoes in its path. Residual sprays, on the other hand, must be applied directly to those areas where mosquitoes are expected to rest during daylight hours.

Mosquito fogging may involve the use of either thermal or cold fogging equipment. Thermal foggers (both portable and vehicle-mounted) use an insecticide which has been mixed with an oil (e.g. diesel, fuel oil) prior to application. The insecticide-oil mixture is vaporized in a special heat chamber of the thermal fogger and, aided by an air blast, the resulting gas condenses when it comes into contact with cooler air, forming a dense cloud of fine droplets which drifts downwind, away from the fogger.

Cold foggers do not use heat to form similar spray droplets. An air blast, coupled with a specially-designed nozzle, breaks the liquid insecticide concentrate into small droplets. Rather than forming a dense, white cloud like the thermal fogger, the cold fogger generates an inconspicuous mist.

Cold foggers (e.g. Leco, MicroGen) are more technologically advanced than thermal foggers (e.g. Tifa, MicroGen). The droplets produced by cold foggers are of a very narrow size range, designed to be optimum in size for killing mosquitoes, and the insecticide flow rate is more precisely-controlled. The net effect is that they destroy more mosquitoes, use less insecticide, and do not 
cost as much to operate.

Other advantages include the fact that they present less of a traffic hazard than the dense white cloud produced by thermal foggers. Also, because the insecticide is much less conspicuous, cold fogs do not bother those people who are opposed to fogging as much as thermal fogs do.

The tiny droplets generated by such foggers are carried downwind through the area to be treated. The droplets, most of which are suspended on air currents drift through vegetation and around obstructions. They impact or impinge on rough surfaces, including mosquitoes. These droplets, whether they impinge or continue drifting downwind, evaporate over a period of hours. If they impinge on the hairs or scales of a mosquito, the insecticide penetrates into the mosquito, causing its death. Such insecticidal fogs are generally effective against mosquitoes for up to about $100 \mathrm{~m}$. Beyond that distance, the droplets usually have become too small through evaporation and have dispersed too widely to impinge on and kill mosquitoes.

There are many brands of thermal and cold foggers (see Appendix) available through such Canadian dealers as KemSan Inc. and Sanex Inc. Abatement officials directing large mosquito control programs in Canada can offer advice on the performance of the various brands available. Dealers should be contacted for detailed information on the models currently available.

Consideration should be given to price, delivery time, staff training and the availability of parts and service. The most efficient foggers are large, heavy-duty units that are mounted in the back of pick-up trucks. Portable foggers may be useful in some situations (e.g., along woodland paths, between buildings) but they are relatively inefficient and labour-intensive.

ULV equipment also can be mounted on aircraft. In a municipal situation, ULV nozzles (e.g., Beecomist nozzles) have been mounted on helicopters for treating large, recreational areas. This approach may be very useful when such areas cannot be treated using ground-based equipment where such equipment might damage wet turf or because the areas are heavily wooded or otherwise inaccessible.

Many different mosquito adulticides are registered for use in ground-based equipment. The insecticides currently registered for this purpose are listed in one of the attached appendices. The choice of adulticide will depend on the spray equipment available, cost and relative human and environmental safety. To make an informed choice, one should obtain technical information on the product from the manufacturer (including specimen labels, material safety data sheets, technical bulletins, and unit prices). As for spray equipment selection, other mosquito abatement officials may be the best source of information on the performance characteristics and problems associated with the various brands and models.

One would expect thermal fogging and cold fogging to be equally effective in controlling $C x$. tarsalis and other vector species provided that the insecticide is applied according to 
label directions, the equipment is accurately calibrated and spraying takes place when mosquitoes are most active (i.e., peaks in activity occur 1 hour before and up to 3 hours after sunset and 0.5 hours before and up to 2 hours after sunrise). It should be noted that, on some days, mosquitoes may be fairly active throughout the day because of warm, humid, cloudy, calm weather conditions. Also, fogging has a flushing action on mosquitoes, stirring even resting mosquitoes to flight, perhaps through a repellent action. However, these conditions cannot be depended upon. It is still best to concentrate the fogging efforts at sunset and sunrise for maximum effect on the mosquito vector population.

A municipality can estimate its requirements for fogging equipment and adulticide by considering the number of $\mathrm{km}$ of streets, lanes and roads that can be fogged, the insecticide flow rate and the vehicle's operating speed. Assuming 6 hours of fogging per night, under optimum weather conditions (i.e. temperatures of $12-28^{\circ} \mathrm{C}$, windspeeds less than $16 \mathrm{~km} / \mathrm{h}$, relative humidities of $35-95 \%$ ), and knowing how often one wants to fog an area, one can calculate the number of fogging trucks and the amount of insecticide required.

Given the opportunity to purchase new mosquito adulticiding equipment, serious consideration should be given to replacing old thermal foggers with new ULV sprayers. The many advantages ULV sprayers have over thermal foggers should not be ignored. Although they may be slightly more expensive to purchase than thermal foggers, they will cost less to operate.

Ideally, fogging trucks should drive perpendicular to the prevailing wind for even dispersal of the fog. This is usually not possible, especially in new suburban areas with numerous crescents and bays. Usually, the trucks must simply follow existing road systems, with unavoidable gaps and overlaps occurring.

During routine mosquito adulticiding operations, it is wise to respect the wishes of those opposed to fogging, leaving their properties and an adequate buffer around them untreated. However, during an emergency adulticiding operation, local authorities may choose to treat all properties alike, advising any persons opposed to spraying to close their windows and stay indoors while spraying takes place.

\section{Residual Spraying}

Residual spraying can have an important role in vector control. Applied to the surfaces of vegetation on which mosquitoes rest, residual sprays usually remain toxic to mosquitoes for one to several days before being destroyed by sunlight, moisture, and / or high temperature. If fogging equipment is unavailable, residual spraying for adult mosquito control should be the next choice for adult mosquito vector control.

Spraying plant surfaces (e.g. shrubs, tree trunks, tall grass, weedy areas (especially close to mosquito breeding sites]) 
will destroy mosquitoes resting on these surfaces over several days. Only water-based emulsions should be used (especially on such high value plants as ornamental shrubs) because oil-based sprays frequently "burn" the foliage of sensitive plant species. Such plants are usually listed on the insecticide container label.

Portable sprayers can be used effectively to apply a coarse spray to dense vegetation in backyards and along paths in parks, golf courses and other recreational areas at times when people are absent. Truck- or trailer-mounted sprayers are most efficient along accessible road, rail and transmission line rights-of way. Aircraft also have been used to apply residual sprays, usually along large, relatively inaccessible, drainage ditches with intermittent stagnant water. Environmentally-sensitive areas (e.g. bird sanctuaries, school grounds) are best omitted from such operations.

Spraying should be done according to approved label directions. Optimum vector control will occur if residual spraying is done when temperatures are between $12-28^{\circ} \mathrm{C}$, winds are low (i.e. less than $16 \mathrm{~km} / \mathrm{h}$ ), and the risk of precipitation, while the residual spray dries on the plant surfaces, is minimal.

\section{Occupational Safety}

Because the personnel involved in insecticide handling, mixing and application will be exposed to insecticide for extended periods of time during the emergency vector control program, it is very important that these people be provided with and use all necessary protective clothing and gear. Specific requirements may vary with the worker's activities and the insecticide's toxicity. Occupational safety and health officials, the manufacturer and the container label will provide advice on what protective measures are necessary.

Generally, applicators should wear protective coveralls, rubber boots, rubber gloves, goggles, and an approved agricultural respirator when exposed to spray mists. Persons mixing insecticides may also wear rubber aprons or rain suits for added protection from spills or splashes. These items usually are readily available from safety supply dealers in major cities.

Also, good personal hygiene practices should be encouraged. Workers should clean their safety gear daily, replacing components as necessary. Clean clothes should be worn each day. Hands should be washed before eating or using the washroom. Showers should be taken before changing into clean clothes. Such precautions will minimize any possible chronic absorption of insecticide into their systems.

\section{Coordination of Control Operations}

As noted above, municipal and provincial vector control operations will rarely, if ever, run independent of each other. The operations must be coordinated for maximum effectiveness. Once the provincial program of aerial ULV application is scheduled for a city or town that has been carrying-out mosquito adulticiding while waiting for the provincial program to get or- 
ganized, that municipality should cease adulticiding and switch over its staff and equipment resources to mosquito larviciding.

This change in operations will avoid unnecessary overlaps in adulticiding efforts, further reduce the potential for virus transmission, avoid possible confusion over liability claims and reduce complaints from those opposed to spraying.

One way in which provinces can encourage cooperation from such municipalities is to agree to pay all of the costs incurred by the municipality in its efforts to reduce local vector populations. Obviously, municipalities would be well-advised to carefully record all expenses they incur which are directly related to emergency vector control even if the work is done before the province agrees to pay all or a portion of the costs involved.

\section{b. Value of Ground-Based Control}

Ground-based adulticiding, using a ULV truck-mounted aerosol generator, is often initiated or increased when an emergency vector control program is considered necessary. Due to the large areas involved in outbreaks of WEE and the urgency with which the treatment must be conducted, ground-based adulticiding can only supplement a larger scale aerial operation.

Weather conditions, vehicle speed, insecticide flow rate, and the optimum droplet size are specific for the equipment and the insecticides selected. Regarding droplet size, the objective is to obtain a narrow range of sizes so that 80 to $90 \%$ of the droplets are 5-20 $\mathrm{u}$ in diameter and have a volume median diameter (VMD) of 10u-17u (Mount et a1. 1975a).

Most bioassays to evaluate the effect of a given adulticide on mosquitoes, using ground-based aerosol generators, have been conducted using caged mosquitoes in open areas. Mosquitoes are placed at various heights above ground, at distances of 50 to $200 \mathrm{~m}$ beyond and perpendicular to the path of the equipment (Mount et al. 1968; Mount et a1. 1975a,b; Rathburn and Boike 1975; Rathburn et al. 1981; Walker and Meisch 1982). The results indicate high levels of mortality. However, the results using caged mosquitoes placed in wooded or protected sites indicate low to medium mortality (Taylor and Schoof 1971; Womeldorf et al. 1973).

Caged mosquitoes placed under shrubs or trees during aerial treatments of ULV propoxur and ULV malathion were also protected from the insecticide droplets, resulting in reduced mortality (Brust 1984; Brust and Ellis 1976b; Ellis and Brust 1982).

Only a few studies have evaluated the effect of groundbased aerosol treatments on natural populations of gravid Culex mosquitoes in urban areas. Results vary from no reduction of egg rafts laid by gravid females of $C x$. pipiens complex and $C x$. restuans, to $50 \%$ reduction for 1 to 4 days following treatment with ULV malathion (Leiser et al. 1982; Strickman 1979). The size 
of the treatment area, the dispersal rate of gravid females, the sample size and the environmental conditions at the time of treatment can affect the level of control. It is therefore important to replicate the experiments on different nights and to treat areas large enough to be representative of an operational program. This appears to have been done in a long term study on the effect of ULV ground-based adulticiding conducted by Geerey et a1. (1983). An analysis of 32 area-wide treatments over 5 years in a Chicago suburb showed that Culex females were reduced by $27 \%$ for 3 nights following the malathion treatments. The reduction during the first night was $39 \%$ and on the second and third nights, $24 \%$ and $18 \%$ respectively.

Natural populations of mosquitoes may escape most of the aerosol treatment due to the protection afforded by tall grassy vegetation, trees, shrubs, hedges, fences, houses and other buildings. When caged Culex spp. were placed $10 \mathrm{~m}$ from a residential street, mosquitoes were readily killed by a ULV application of malathion. However, when cages were placed $115 \mathrm{~m}$ from the street, in vegetation along alleys, the mosquitoes survived. The mean mortality in caged mosquitoes placed at various distances from the truck-mounted aerosol generator in the urban area of South Bend, IN was $26 \%$ (Leiser et al. 1982).

Insecticide droplets must make direct contact with mosquitoes if the application is going to be effective in killing adults. The best results would be expected when mosquitoes are in flight. Mosquito flight activity decreases sharply during the darkest part of the night (Nelson and Spadoni 1972), and adulticiding may be less effective during these hours.

In Winnipeg, Manitoba, during 1977 to 1984, where an average of an estimated $150 \mathrm{sq}$. mi. were treated with ULV ground-based adulticiding equipment in 7 nights (E1lis,pers.comm.) the reduction in trap counts of $C x$. tarsalis has been calculated to be $68 \%$ following a treatment (Raddatz 1985). This reduction was not based on experimental results of mosquito mortality following adulticiding, but was calculated rather as a factor in the trap count model that gave the best estimate of parameters that explained the variables in the trap counts during 7 summer seasons in Winnipeg. Because a minimum of 7 nights were needed, and because of unsuitable weather conditions, it usually required more nights to treat the Winnipeg area. There was no way to estimate

the $\%$ control on different nights after treatment. The estimate of $68 \%$ control appears high compared to that shown for the Chicago area by Geery et al. 1983).

\section{c. Assessment of Repellents}

The alternatives that are available to protect people against mosquito-borne encephalitis include direct mosquito control using insecticides and/or host protection by means of 
personal protection, vaccination, screening, behaviourial modification, suitable clothing, and sanitation. The term, "personal protection", is defined here as the use of an approved insect repellent by a person to prevent mosquito bites. Following an introduction to the insect repellents registered in Canada for protection against mosquitoes, the safety and effectiveness of DEET, the active ingredient common to most repellents, will be reviewed.

DEET is an effective repellent against more than 20 genera of insects and related arthropods. A wealth of scientific literature has been published on DEET on the chemistry, toxicology, and effectiveness of DEET over the past 36 years. Those interested in an in-depth review of DEET may wish to consult the bibliography prepared by Rutledge et al. (1978) and the DEET pesticide registration standard prepared by the U.S. EPA (Panetta et al. 1980). The latter document is a comprehensive review of the toxicology and efficacy of DEET.

Repellents must provide a $2+$ hour protection time before they can be registered for use in Canada. Several different active ingredients, alone or in combination, are used in repellent formulations. Repellents are registered with Agriculture Canada to protect against ticks, black flies, biting midges, horse flies, deer flies and mosquitoes. The per cent active ingredient(s) present in a formulation of repellent may vary considerably from product to product. Domestic class insect repellents can be purchased in a variety of forms including liquids, pressurized aerosols, sticks, foams, towellettes or wipes and impregnated fabrics. Given the large number and variety of repellents that are available to consumers for direct application to their skin, their safety and effectiveness need to be re-assessed on a regular basis to ensure that they meet current standards.

It is beyond the scope of this section to review the safety and effectiveness of every active ingredient used in mosquito repellents. However, DEET, alone or in combination with one or more of the other active ingredients, is the primary ingredient most commonly found in repellents. Thus, this assessment will be limited to DEET as a single active ingredient in a repellent product. It is not intended to be an exhaustive review. Rather, it attempts to address those concerns raised by public health officials over the widespread use of repellents during an encephalitis outbreak.

\section{Toxicology of DEET}

DEET has been marketed in Canada for over 30 years. In the U.S.A., an estimated 50-100 million people use a repellent each year. Most repellents sold contain DEET as the sole or primary active ingredient. Extrapolating from these U.S. estimates, 5-10 million people probably use a repellent each year in Canada. 
There have been numerous anecdotal reports of skin irritation following applicationof repellents, several cases of toxic systemic reactions from repeated use of products containing DEET (mostly children; most recovering but some ending in death) and several deaths following ingestion of products containing DEET (MMWR 1989).

Adverse effects are always attributed to DEET, even though the products involved may have been a mixture of DEET and several other active ingredients. The safety record of repellents is excellent considering the numbers of persons using them annually to obtain relief from the annoyance and bites of insects and ticks and to protect against arthropod-borne disease. On the other hand, any adverse effects, even in a very small segment of the human population, should underline the need for carefully conducted toxicological and epidemiological studies on all of the active ingredients used in repellents and the need for precautions to be followed explicitly in their use.

Dermally-applied DEET is rapidly absorbed through the skin and excreted in the urine of laboratory animals. Thus, bioaccumulation is expected to be minimal. DEET is excreted secondarily via the feces, lacrimal glands and the nasal mucosa.

Rates of excretion in urine varies with the species (Blomquist and Thorsell 1977):

- $80 \%$ excretion in 24 hours in guinea pigs;

- $90 \%$ excretion in 8-40 hours in mice; and

- $68 \%$ excretion in 24 hours in rats.

Studies of human metabolism of DEET are not very complete. Although up to $49 \%$ of dermally-applied DEET is lost through evaporation (Spencer et al. 1979), excretion rates are variable among the few individuals studied. For example, when $52 \mathrm{mg}$ of DEET was applied to the forearms of 4 human volunteers, $13.3 \%$ was recovered in the urine in 24 hours, $15.3 \%$ by 48 hours and $16.7 \%$ by 12 days (Feldman and Maibach 1970). Using 1 volunteer Blomquist and Thorsell (1977) showed that, when 2 applications of $0.12 \mathrm{mg} / \mathrm{kg}$ body weight was applied to the forearm, $5.5 \%$ was recovered in the urine in 48 hours after the first application and $3.8 \%$ was recovered in 48 hours after the second application. In a third study (Markina and Yatsenko, 1971), 20-40\% DEET creme formulations were applied to 15 volunteers. No toxicity was reported after 30 days of daily application. Most of the DEET absorbed through the skin $(>96 \%)$ is excreted in the urine within 24 hours.

A small percentage of individuals exhibit skin irritation and allergic effects from use of 15-100\% DEET formulations. Skin irritations, rashes, hives and blisters as well as eye, nose and mouth irritations have been reported (Hollebone pers. comm.). 
Repeated applications of DEET may cause drying and irritation of the skin, especially around the nose area (Ambrose et al. 1959; Phillips et al. 1972). There are reports of illnesses, including nausea and dizziness, after repeated applications of DEET. Neurological events associated with the repeated use of DEET have been observed. In 1989, 5 reports of generalized seizures, occurring 8-24 hours after DEET use, were investigated in New York and Connecticut where DEET use has increased due to the concern over Lyme disease (MMWR 1989). Four cases were 3-7 year-old boys; one was a 29 year-old man. All recovered. It is not known whether or not their illnesses were directly attributable to DEET use.

Some trans-placental effects may occur in pregnant small animals, but the reproductive effects, if any, in humans are unknown. DEET may decrease implantations but does not appear to be a spermatogenetic agent.

Although the general toxicity of DEET is low, it appears that the data available on DEET (and other active ingredients of repellents) and human toxicology are not adequate to assess clearly the chronic or sub-chronic effects of repeated doses of DEET. The U.S. EPA has identified a number of data gaps (Panetta 1980). Exposure studies to determine the effects of repeated use of DEET formulations should be initiated.

Several precautions should accompany any promotion of repellents as a means of personal protection during an outbreak of mosquito-borne encephalitis. The following list of precautions is based on one given in a recent MMWR (1989):

- Apply repellent only to exposed skin or clothing.

- Avoid applying high-concentration products to the skin, especially children.

- Avoid inhaling and ingesting repellents and getting into the eyes.

- Wear long sleeves and long pants to minimize the skin area that must be treated.

- Avoid applying repellents to children's hands which are likely to contact their eyes or mouths.

- Never apply repellents to irritated skin or wounds.

- Use sparingly; one application will usually last 2-8 hours. 
- Wash repellent off the skin after coming indoors.

- If an adverse reaction occurs, wash treated skin and call a physician; take the repellent to the physician.

The effectiveness of a given insect repellent may be affected by a number of inter-related factors including weather (ambient temperature, wind, humidity, etc.), mosquito biting pressure, mosquito species, user physiological characteristics and state (i.e., subject susceptibility), formulation ingredients and characteristics (absorption rate, evaporation rate, etc.), loss through abrasion, and application rate. Given all the possible combinations of factors, it is understandable that different products give different levels and periods of protection, depending upon the unique conditions occurring when they are evaluated. This variability is reflected in the technical and scientific literature (Panetta et al. 1980).

DEET loss, through abrasion, evaporation or washing off the skin or through absorption into the user's body, will result in reduced effectiveness. Increased wind velocity, increased temperature, rain and sweat can decrease efficacy by $50-90 \%$ (Khan et a1. 1973; Gilbert et al. 1957; Goulk et al., 1971; Schieffer et a1. 1976). Cutaneous absorption of DEET also may reduce efficacy (Mailbach et al. 1974).

The minimum effective dose on human skin required to repel caged Aedes aegypti appears to be 0.02-0.2 mg DEET/cm 


\section{ENVIRONMENTAL MONITORING}

a. Spray Dispersal

Whenever pesticides are sprayed, especially from aircraft, some material inevitably drifts outside the target zone. The amount of material lost from the target zone depends on the application technology, weather, topography, and the pesticide formulation. Different spray techniques deliver different ranges of droplet sizes, with ultra-low-volume equipment typically delivering droplets with a mass median diameter in the 70-100 um range (Randall 1977). The size of spray droplets is an important factor in determining the distance they drift, with droplets larger than 100 um drifting very little unless the wind during application is very strong. However, after emission from the nozzle droplets begin to evaporate, and they decrease in size.

The theoretical development of relationships between droplet diameter and fall time has been given by Seymour (1969), who illustrated the time for a droplet of given initial size to be reduced to zero. For example, a 100-um droplet of water would last only about 6 seconds at $30 \%$ relative humidity (RH), about 11 seconds at $50 \% \mathrm{RH}$, and about $12 \mathrm{sec}$ at $70 \% \mathrm{RH}$. Smaller droplets and the vapour produced from them move both horizontally and vertically with air motion, and may drift off the target zone, sometimes for quite long distances, leaving the target zone to recieve less than the ideal amount of material which was emitted from the sprayer. For example, Armstrong (1977) reported that 4 to $100 \%$ of Orthene emitted by a spray aircraft was deposited on spray blocks. When propoxur was applied by aircraft to Winnipeg in 1975 and 1981, generally less than $20 \%$ of the pesticide emitted from the aircraft could be accounted for by measurements of deposits (Manitoba Clean Environment Commission, 1982). Ware et al. (1984) monitored deposits of fenvalerate applied aerially to cotton and found that off-target deposits declined quickly with distance from the target zone out to about $100 \mathrm{~m}$, and then less strikingly out to at least $400 \mathrm{~m}$ downwind, where they were still 1-10\% of those in the target zone. The actual distance the drifting material can travel has no practical limit if the material is stable to decomposition. It may be carried long distances with air mass movements either in the gas phase or adsorbed to suspended particles. For example, DDT from forest spraying in New Brunswick and Maine was thought to have been the source of DDT detected in rainwater in the Magdalen Islands (Pearce et al. 1978). Sheehan et al. (1987) have summarized the topic of off-target drift (their chapter 5), and Table 1 is abstracted from their summary.

Within the target zone, the surface to volume relationship of receiving areas will determine to a large extent the concentration of pesticide received. Aerial sprays are generally 
applied to deliver the desired amount to a two-dimensional surface within the spray zone, but the pesticide is actually dispersed in three dimensions. For example, in the instance of a body of water, the simplest case is one in which the water is mixed throughout and so the greater the volume per area of sprayed surface, the greater the opportunity for dilution. In such a simple case, a large shallow pool would experience a higher concentration than a small deep pool with the same volume. The surface film itself provides a concentrating mechanism for movements of the pesticide either from air to water or from water to air, and organisms using the surface habitat are particularly prone to exposure.

Once deposited pesticides re-volatilize from surfaces such as soils or foliage at rates that depend on the temperature, wind, vapour pressure of the pesticide, the formulation, and the nature of the surface (Wheatley, 1973). The pesticides may then be dispersed further throughout the environment. For example, several pesticides including chlorpyrifos and malathion have been reported recently in fog droplets at concentrations much higher than those expected on the basis of Henry's Law constants (Glotfelty et al. 1987), and so these pesticides can be dispersed with fog droplets.

While the phenomenon of off-target drift has been documented many times, the concentration of pesticide reaching non-target zones is clearly subject to the mathematics of dilution, and exposure of animals or plants outside the target zone will almost always be much less than exposure within the target zone. Other modes of application would be expected to generate less offtarget drift than aerial application.

\section{b. Effects on Wildife}

The effects on wildlife are of two general kinds. Firstly there are the direct toxicological effects due to exposure to the pesticide being applied, and secondly there are the indirect or secondary effects of changes in one species on the biology of another (Hurlbert 1975). With regard to the direct toxicological effects, the critical parameters are exposure and toxicology.

Exposure is related to the amount of chemical an organism takes in per unit of time, and the duration over which the intake occurs. Toxicology is related to the chemical and physical properties of the pesticide and to the means by which exposure takes place (dietary, contact, inhalation, etc.). Discussion of the effects of a pesticide needs to start with the environmental chemistry of the pesticide since that will determine the exposure. However, this subject has a very large literature and will be treated only superficially here.

Most pesticides used for mosquito control in North America are designed to remain active for only short periods after application. A list of pesticides registered for mosquito control 
in Canada is given in Appendix I, and application rates range up to $550 \mathrm{~g} \mathrm{ai} / \mathrm{ha}$. An example of rapid 'disappearance' of three organophosphorus insecticides (temephos, Reldan, and chlorpyriphos) from the water column of small polyethylene-lined pools was given by Hughes et al. (1980), who found that over $90 \%$ of all three compounds frequently 'disappeared' in less than 2 days. The polyethylene apparently acted as a sorbent for the compounds, particularly chlorpyrifos, to first remove it from the water, and then gradually to supply it back and contribute to a residual effect. MacKenzie et al. (1983) followed the loss of temephos from mosquito breeding ponds in Ontario and found that residues in the water declined over $24 \mathrm{hr}$, but that residues in bottom sediment increased over that period and then declined over the following several days. Residues derived from temephos were still present in at the end of the sampling period, particularly in sediments, but they consisted principally of sulfoxide and sulfone metabolites rather than the parent pesticide.

Rapid disappearance can be explained by an array of processes which act to remove or change the pesticide after it is emitted, including photolysis, hydrolysis, adsorption, volatilization, and metabolism by different organisms. In water hydrolysis is often important in destroying the parent compound (and in creating new products), and the rate of hydrolysis is often described as a 'first-order' process in which the amount hydrolyzed at any instant depends on the amount of parent compound present at that time. This gives rise to an exponential decline in the amount remaining, often described by the half-life of the compound. For example, Marshall and Roberts (1978) summarized studies on the hydrolysis of chlorpyrifos, and cited earlier work showing the dependency on $\mathrm{pH}$ and temperature, as shown in Table 2. At constant $\mathrm{pH}$, hydrolysis was much faster at higher temperature, and at constant temperature, hydrolysis was much faster at more alkaline $\mathrm{pH}$.

The most widely used measure relating exposure and direct toxic effects is the exposure that is acutely lethal under defined test conditions. With terrestrial animals this is usually the single dose given orally that results in the death of half the treated animals over some arbitrary but defined observation period. With aquatic animals, the "dose" is usually expressed as the concentration supplied in the water rather than the amount actually taken into the animal.

Chemical pesticides generally have little or no specificity

for the target pests; if specificity is achieved it is through the application which attempts to maximize exposure of the pest without at the same time unduely exposing non-target species. Where the habitat of target and non-target species overlaps, however, applications will reach both. The acute toxicities of a few common pesticides to Culex mosquitoes, along with toxicities 
to non-target midges are presented in Table 3 (abstracted from Sheehan et al., 1987). The acute toxicities are similar for both groups, and is clear that these pesticides do not discriminate between the mosquitoes and the midges.

Some additional data on toxicity of common pesticides to other non-target invertebrate fauna are listed in Table 4, from Mayer and Ellersieck (1986).

In contrast to the conventional pesticides, some of the newer insect growth regulators appear to be selectively more toxic to target pests than to non-target fauna. Table 5 (abstracted from Brown 1978) is a list of the toxicity of methoprene and diflubenzuron to mosquito larvae and to several non-target taxa. Methoprene in particular is much more toxic to the mosquito larvae than to the other invertebrates tested.

The safety of fish and wildlife is perhaps of greater immediate concern to more people than that of invertebrates. Some concentrations acutely lethal to rainbow trout are given in the Table 6 below and oral doses toxic to laboratory rats are given in Table 7 .

The more subtle effects of pesticide exposure on non-target animals are obviously much more difficult to demonstrate, particularly in natural populations. However concepts and methodologies are beginning to emerge to detect signs of stress in ecosystems (Levin et al. 1983; Rapport et al. 1985). Some of the clearest cases have been with older organochlorine compounds (see review by Ripper 1956), but these will not be discussed here because they are no longer in use for arbovirus vector control in Canada. There are also convincing cases with organophosphours insecticides, and some of these are discussed below.

Organophosphorus and carbamate insecticides act on synaptic transmission by inhibiting acetylcholinesterase enzyme activity, and a number of cases of enzyme inhibition in non-target organisms has been recorded. For example, Lockhart et al. (1985) reported inhibition of about $75 \%$ of the brain acetyl cholinesterase activity in young fish stocked in shallow ponds sprayed with malathion for control of mosquitoes. The fish in this case (no predators) were apparently not harmed permanently because they survived. Their enzyme activities recovered to near pre-treatment levels in about two weeks, and the only evidence of ecological harm was a temporary cessation in growth.

Some more subtle effects of cholinesterase inhibitors have been noted in higher integrative functions. For example, exposure of young Atlantic salmon to fenitrothion increased their vulnerability to predation by brook trout (Hatfield and Anderson 1972). In rats, reduction of brain cholinesterase activity to 40 $60 \%$ of normal resulted in increased numbers of mistakes in solving problems presented by a maze (Banks and Russell 1967). Treatment of fidler crabs Uca pugnax with temephos resulted in 
impairment of their ability to escape predation by birds (Ward et a1. 1976).

There have been reports of bird kills after applications of organophosphorus insecticides and brain cholinesterase activities have been used as an aid in diagnosis. Diazinon applied to a golf course was implicated in the death of a small number of Canada geese in Missouri (Zinkl et al. 1978). Brain cholinesterase activities were about one third of those from control birds with a comparable brain storage history.

At the population level one of the most convincing examples of effects of insecticide applications acting on a species other than the target species has been the proliferation of prey after removal of predators. Killing non-target zooplankton has frequently produced an upsurge in algal numbers due to decreased cropping (Hurlbert 1975), and this has been reported following applications of insecticides for mosquito control in Canada (Hughes et al. 1980).

Perhaps the best-documented effect of pesticide spraying has been the development of pest populations resistant to the pesticides. In a recent review of this problem, worldwide there are now 447 species of insects and mites resistant to at least one insecticide (Georghiou 1986). The order of insects with the greatest propensity to develop resistance, unfortunately, includes the common vectors of viral infections, namely the Diptera, with 156 resistant species. Furthermore, of these 156 species of resistant Diptera, 132 are of medical or veterinary importance, while 23 are of agricultural importance. Many of these species are resistant to several classes of insecticides, with the current accounting as shown in the Table 8 .

\section{c. Effects on Humans}

The effects of pesticide applications are of three general types: benefits to human health and economic prosperity inherent in the control of disease vectors; direct toxicological effects on people exposed to the pesticides; indirect effects on people through changes in ecosystems.

Perhaps the clearest case of the benefits of vector control to people is DDT in tropical regions. Metcalf (1973) concluded that DDT campaigns eradicated malaria in 37 countries with a population of 728 million people, and gave partial or complete control in 80 more countries with 618 million people. In India, for example, cases of malaria declined from 100 million annually in 1933-35 to 150,000 in 1966, and deaths fell from 750,000 per year to 1,500 .

It is not uncommon for pesticides used for biting fly control to reach people through pathways independent of uses against biting flies. Gartrell et al. (1986) tabulated the quantities of pesticide residues found in typical U.S. food items as shown in 
Table 9. Obviously people consuming common food items are experiencing ongoing low levels of dietary exposure to a range of pesticides including some used for biting fly control. Given multiple exposure pathways leading to people, it seems unlikely that effects of pesticides used for biting fly control will be separable from effects of the same pesticides used for other puropses, except in the instance of acute effects associated with known local applications.

For obvious reasons most information on the direct toxicological effects of pesticides on people comes from accidental exposures, occupational exposures of people who work with pesticides, and occasionally suicides or homicides. However, there have been a number of experiments with human volunteers, many of which have been listed by Hayes (1975). For example, Laws et al. (1967) gave oral doses of temephos to volunteers (prison inmates in Puerto Rico) at up to $256 \mathrm{mg} / \mathrm{man} /$ day for 5 days, or 64 $\mathrm{mg} / \mathrm{man} /$ day for 4 weeks with no clinical symptoms or side effects noted, and no effect on red blood cell or plasma cholinesterase activities. Similar experiments with methoxychlor given orally to human volunteers (prison inmates in Alabama) were reported by Fabian et al. (1971). Methoxychlor given at rates up to 200 times the levels permitted by the U.S. Food and Drug Administration had none of the effects expected on the basis of prior experiments with rats. Exposed rat experienced changes in fatty acid distribution, inhibition of spermatogenesis, and alterations in the ultrastructure of intestine and liver, and these effects were sought but not found in the people.

Survey data on people who work with pesticides have not revealed unusual incidences of diseases, as compared with the population not exposed to pesticides occupationally, however, pesticide workers did suffer an unusually high frequency of death by trauma (Morgan et al. 1980). During the 1960s, medical students at the University of Montreal interviewed members of farm families where pesticides were used on apple orchards. About one third (520 out of 1661 interviewed) complained of symptoms (mainly eye irritation, fatigue, and headache) for a short period (up to 7 days) after spraying (Jegier 1969). Malathion has been shown to induce contact sensitization in some people (Milby and Epstein 1964).

It has been argued that substantially over 10,000 people are killed annually worldwide by exposure to pesticides, generally through exposure during application (Loevinsohn 1987), but little of this would be expected to be associated with biting fly control in North America.

Recently published Federal guidelines for the water quality do not give permissable quantities for any of the pesticides used commonly for mosquito control (Canadian Water Quality Guidelines 1987). 
One effect which should not be underestimated is the fear people express when exposure to pesticides seems unavoidable. This is analagous to fear of exposure to radiation, and it is noteworthy that a U.S. court considering the re-opening of the Three Mile Island nuclear plant ruled that the Nuclear Regulatory Commission must concern itself with popular fears about the reactor, regardless of whether or not the fears have a rational basis (Marshall 1982). People often associate high risk with the uses of pesticides. The risks associated with 30 different activities or technologies were ranked by four different groups of people, and pesticides ranged from 4th to 15th. 'Experts' ranked pesticides 8 th behind motor vehicles, smoking, alcohol, handguns, surgery, motorcycles, and x-rays (Slovic 1987).

Indirect effects on people through pesticide actions are much more difficult to document, except for the economic or public health effects for which the application was made. The common examples include contamination of fish and wildlife leading to intake by people with possible biological effects on the people (Jacobson and Jacobson 1988) but these examples generally refer to stable halogenated compounds. However, the methodologies emerging to deal with older compounds may also offer promise of detecting subtle effects of less stable pesticides used for biting fly control. Also of concern to people are effects of pesticides on non-target species of interest to humans for aesthetic or economic reasons, as discussed earlier. 
Table 1. Off-target deposits of aerially applied pesticides. Deposit as per cent of application rate.

\begin{tabular}{lccc}
\hline Pesticide & $\begin{array}{c}50 \mathrm{~m} \\
\text { downwind }\end{array}$ & $\begin{array}{c}100 \mathrm{~m} \\
\text { downwind }\end{array}$ & $\begin{array}{c}500 \mathrm{~m} \\
\text { downwind }\end{array}$ \\
\hline malathion & 6.5 & 1.5 & 0.04 \\
azinphos methy1 & 10.5 & 0.9 & 0.2 \\
methoxychlor & 1.2 & 0.9 & 0.09 \\
& 1.1 & 0.9 & 0.1 \\
fenvalerate & - & 0.5 & 0.15 \\
\hline
\end{tabular}

Abbreviated from Table 5.3 of Sheehan et a1. 1987 
Table 2. Rate of hydrolysis of chlorpyrifos in phosphate buffered (0.02 M) distilled water at $15 \mathrm{C}$ to $35 \mathrm{C}$, and an initial concentration of $0.12 \mathrm{mg} / \mathrm{L}$.

\begin{tabular}{llcc}
\hline $\begin{array}{c}\text { Temperature } \\
\text { deg. C }\end{array}$ & $\mathrm{pH}$ & $\begin{array}{c}\text { half-life } \\
\text { (days) }\end{array}$ & $\begin{array}{c}\text { duration of experiment } \\
\text { (days) }\end{array}$ \\
\hline 15 & 8.1 & 53.5 & 29 \\
25 & 8.1 & 22.9 & 28 \\
35 & 8.1 & 4.5 & 12 \\
& & & 29 \\
15 & 6.9 & 99.6 & 28 \\
35 & 6.9 & 35.2 & 26 \\
15 & 6.9 & 11.5 & 29 \\
25 & 4.7 & 206.3 & 28 \\
35 & 4.7 & 62.8 & 28 \\
\hline
\end{tabular}

From Marshall and Roberts (1978), Table 2.2. 
Table 3. Comparative toxicities of chemical pesticides to mosquitoes and other non-target aquatic invertebrates.

24-hr $\mathrm{LC}_{50}(\mathrm{ug} / \mathrm{L})$

\begin{tabular}{lcc}
\hline Insecticide & $\begin{array}{l}\text { mosquitoes } \\
\text { Culex spp }\end{array}$ & midges \\
\hline chlorpyrifos & $0.3-1.2$ & $0.5-7.0$ \\
malathion & $3.4-68.0$ & $7.0-100$ \\
methoxychlor & $8.9-18.9$ & $1.6(96 \mathrm{~h})$ \\
deltamethrin & $0.02-0.23$ & $0.02-0.29$ \\
carbaryl & $75-418$ & $10(48 \mathrm{~h})$ \\
\hline
\end{tabular}

From Sheehan et al. 1987 
Table 4. Acute (96-hr) toxicity of several pesticides to nontarget aquatic invertebrates.

\begin{tabular}{|c|c|c|}
\hline & $\begin{array}{l}\text { Gammarus } \\
\text { lacustris }\end{array}$ & $\begin{array}{l}\text { Pteronarcys } \\
\text { californica }\end{array}$ \\
\hline malathion & - & 10 \\
\hline chlorpyrifos & 0.11 & 10 \\
\hline propoxur & 34 & 18 \\
\hline temephos & 80 & 10 \\
\hline methoxychlor & .80 & 1.4 \\
\hline
\end{tabular}

* Mayer, F.L., Jr., and M.R. Ellersieck (1986). 
Table 5. Acute toxicity of methoprene and diflubenzuron to aquatic invertebrates. *

Species

$24-\mathrm{hr} \mathrm{LC}_{50}(\mathrm{ug} / \mathrm{L})$

diflubenzuron methoprene

$\begin{array}{lcc}\text { Mosquito (Aedes) } & 0.5 & 0.008 \\ \text { Clam shrimp (Eulimnadia) } & 0.15 & 1000 \\ \text { Tadpole shrimp (Triops) } & 0.8 & 5000 \\ \text { Cladoceran (Daphnia) } & 1.5 & 900 \\ \text { Mayfly nymph (Callibaetis) } & 50 & \ldots \\ \text { Dragonfly nymph (Orthemis) } & 50 & 20000 \\ \text { Midge (Chironomus) } & 10 & 10 \\ \text { Backswimmer (Notonecta) } & 10 & 1200 \\ \text { Protozoan (Paramoecium) } & \ldots & 1250 \\ \text { Amphipod (Hyalella) } & \ldots & 1250 \\ \text { Water boatman (Corisella) } & \ldots & 1650 \\ \text { Beetle larva (Hydrophilus) } & 100 & 50000 \\ \text { Copepod (Cyclops) } & 200 & 4600 \\ \text { Seed shrimp (Cypicercus) } & 500 & 1500\end{array}$

* From Brown (1978) p.150. 
Table 6. Concentrations of several pesticides acutely lethal to rainbow trout in 96 -hour exposures."

\begin{tabular}{lccc}
\hline Pesticide & $\begin{array}{c}\text { Fish weight } \\
(\mathrm{g})\end{array}$ & $\begin{array}{c}\text { Temperature } \\
(\mathrm{C})\end{array}$ & $\begin{array}{c}\mathrm{LC}_{50} \\
(\mathrm{ug} / \mathrm{L})\end{array}$ \\
\hline malathion & 1.4 & 12 & 200 \\
chlorpyrifos & 1.4 & 13 & 7.1 \\
propoxur & 1.2 & 13 & 8200 \\
temephos & 1.3 & 12 & 3490 \\
methoprene & 0.6 & 12 & 1600 \\
methoxychlor & 1.2 & 12 & 62 \\
diflubenzuron & 1.0 & 10 & $>100$ \\
\hline
\end{tabular}

* Data from Johnson and Finlay (1980) 
Table 7. Acute oral toxicity of several pesticides to laboratory rats.

\begin{tabular}{lll}
\hline Pesticide & \multicolumn{1}{c}{$\begin{array}{c}\text { Toxicity } \\
\left(\text { Oral } L D_{50}\right)\end{array}$} & \multicolumn{1}{c}{ Reference } \\
\hline malathion & $1375 \mathrm{mg} / \mathrm{kg}$ & Hayes (1975) \\
chlorpyrifos & $82-245 \mathrm{mg} / \mathrm{kg}$ & $\begin{array}{l}\text { Marshal1 \& } \\
\text { Roberts (1978) }\end{array}$ \\
propoxur & $80-191 \mathrm{mg} / \mathrm{kg}$ & $\begin{array}{l}\text { National } \\
\text { Research } \\
\text { Council(1982) }\end{array}$ \\
temephos & Canamid (1970) \\
methoxychlor & $1000 \mathrm{mg} / \mathrm{kg}$ & $\begin{array}{l}\text { Cyanardner \& } \\
\text { Gailey (1975) }\end{array}$
\end{tabular}


Table 8. Numbers of species of Diptera resistant to several classes of insecticides.

\begin{tabular}{cl}
$\begin{array}{c}\text { Number of species } \\
\text { of Diptera }\end{array}$ & $\begin{array}{c}\text { Class of insecticide } \\
\text { insects resist }\end{array}$ \\
\hline 108 & DDT \\
62 & Cyclodienes \\
11 & Organophosphorous \\
10 & Carbamates \\
0 & Pyrethroids \\
1 & Fumigants \\
\hline
\end{tabular}

Retabulated from Georghiou (1986) 
Table 9. Daily intake of selected pesticides calculated from residues in typical U.S. foods.

Pesticide

Average daily intake (ug/day)

carbaryl

0.825

chlorpyrifos

0.241

malathion

16.8

methoxychlor

0.249 


\section{PUBLIC INFORMATION}

a. Information Program

Objectives

The objective of the emergency public information program is to provide complete, current and factual information to the target

audiences on all aspects of the encephalitis outbreak. Given timely and accurate information, the media and the public may more readily support government actions. The following areas require special attention.

Information on the Disease

The disease, whether it is St. Louis Encephalitis (SLE) or Western Equine Encephalitis (WEE) or some other mosquito-borne encephalitis, must be described in terms understandable and specific to the target audience. For example, medical doctors will require information on the diagnosis and treatment of encephalitis whereas mothers with young infants will require information on recognizing the symptoms of the disease in babies. Other audiences, such as the news media, may wish a historical perspective on the disease.

In addition, the general public will require a basic overview of the disease, including such aspects as what the disease is, how it affects people, the typical symptoms of the disease, how the disease is transmitted, how the level of risk is monitored, what people can do to minimize the risk to themselves and members of their family, what measures can be taken to reduce transmission of the disease, and so on.

Information on Vector Control Measures

Any large-scale disease control program undertaken by the government will generate considerable media attention, especially on a regional basis. The news media and their audiences will want to know, in as much detail as possible, what measures are being taken to minimize the risk of disease transmission. They will want to know who is directing the control operations, what specific operations are involved in emergency mosquito control, and what are the pros and cons of the insecticide that is being used to control the vector mosquitoes.

Control Officials

People want to know who is directing the emergency mosquito control program. "Control officials" is a collective term which includes the persons directing the control operations, from a policy point-of-view (i.e. elected officials) and from a technical point-of-view (i.e. field operations personnel). The ultimate decision makers (including the decision to initiate control measures and what scale of operation) are the elected officials, including the Minister of Health and the Minister responsible for emergency measures. Other members of Cabinet may be involved in the approval of funding, waivers of Acts and Regulations, defending government actions, etc. The key decision maker is the Minister of Health and that person will be the focus of public and media 


\section{attention.}

The person(s) directing the emergency mosquito control operations in the field should be technically-qualified to oversee the operations base (usually at the airport), recommend applicators, insecticide, insecticide suppliers, etc. They should be experienced in mosquito abatement, knowing when conditions are optimum for mosquito control. They should be able to liaise with elected officials (particularly the Minister of Health), senior administrators, suppliers and staff at the operations base. Ideally, the person directing the program will have successfully supervised similar, large-scale mosquito control operations in the past and have the confidence of the public.

This person's decisions will have considerable impact on the success of the disease control program. Although some media exposure will be necessary, it should be kept to a minimum to allow this person to carry out the work without the added pressure of dealing with a sometimes aggressive and adversarial media.

\section{Application Method}

The public will want to know that the government is taking all reasonable steps necessary to minimize the health risks associated with the disease as quickly as possible. The media will want to know that the disease control operations are done using the most suitable methods and materials available. The most efficient and economical means of wide-area mosquito vector control is the aerial application of insecticide.

The spray operations should be done by experienced aerial applicators, using large, multi-engine aircraft. Large aircraft (e.g. DC-6) can carry sufficient insecticide to treat a small city during one flight. Two- or, preferably, 4-engine aircraft also provide that extra margin of safety necessary for low-level flights over developed urban and rural centres.

The optimum method of adult mosquito control is the application of very small droplets of insecticide over the area to be protected. In theory, these tiny droplets slowly drift through the area where mosquitoes are flying or resting. A few droplets, impinging on the body of the mosquito, are enough to kill the mosquito. Such ultralow volume applications (abbreviated ULV) require relatively little insecticide per unit area to be effective.

However, many factors may reduce the overall effectiveness of the ULV application. Control will never be 100\%. Changing wind conditions during the period of aerial application may cause the ULV droplets to be unevenly dispersed over the control zone. Heavy vegetation (e.g. thick bush, tall, dense grass) and dense concentrations of buildings and fences may filter out many of the droplets and provide cover for some of the mosquitoes. In some cases, monitoring of mosquito mortality may indicate that re-treatment is necessary. 
Insecticide Used

Inevitably, the government will have to defend, first, the largescale use of insecticide and, second, the specific insecticide chosen for use. Depending upon the number of confirmed human cases of encephalitis at the time, the defence may be relatively simple or complex. Foremost in minds of all the target audiences will be the question, "Is this insecticide safe?".

This general question will have a multitude of facets. Is it safe to breathe? Will it cause cancer? Should I keep the kids indoors? Will it ruin my garden vegetables? Will it hurt my flowers? Is it safe to pets? Will it mar my (very expensive) car finish? Should we cancel the baseball game? Can we still have our outdoor wedding party? Will it kill the butterflies? Will it kill my bees? These and dozens of other questions centre on the question of the safety of the insecticide to human health and environmental acceptability.

Obviously, these questions will be asked by the public and an information package on this topic should be prepared beforehand to answer at least the most commonly-asked questions. Much of this information is available from the insecticide manufacturers and can readily be obtained from them and from other sources such as the federal departments of Agriculture and Health and Welfare.

The key point here is cost-benefit. In simple terms, are the potentially harmful effects of the insecticide more or less desirable than the potentially harmful effects of the disease? On one side of the equation, the monetary costs associated with spraying can be tallied (including the costs of the insecticide, the insecticide application, and any damage associated with the application (e.g. bee kill]). On the other side, one can estimate the number and ages of persons who might contract the disease and the costs associated with their health care and lost earning potential. Unfortunately, many of the costs and benefits, on both sides of the equation, are either intangible or speculative and, thus, their defence is open to criticism. Nevertheless, knowing, for example, that $\$ 1$ million worth of dead bees is equivalent to the life. long, health care costs associated with one infant who becomes paralysed or mentally retarded from the disease may provide some perspective.

\section{Information on Personal Protection}

Practical advice on personal protection from mosquito bites, to people living in regions where mosquito-borne encephalitis is possible, should be provided whenever there is a potential disease outbreak as determined by vector/virus surveillance. Personal protection is simply taking precautionary steps to minimize being bitten by mosquitoes. These range from adjusting personal schedules or activities to avoid peak periods of mosquito activity or areas of high mosquito abundance, to covering exposed skin with clothing and wearing mosquito repellents. Minimizing the number of mosquito bites minimizes the risk of virus transmission from the infective mosquito to people. 
Repellents containing N, N-Diethyl meta-toluamide (DEET) are effective against mosquitoes. There are numerous formulations available, allowing for personal preferences. Repellents that contain Dimethyl phthalate are also effective against mosquitoes, and are often preferred for personal (less oily; DEET disolves hard plastic objects) or health (allergy) reasons. Repellent-impregnated, mesh clothing is available for persons who do not wish to wear repellent directly on their skin. Infants, who should not be treated with repellents, may be kept indoors or protected with mosquito netting when they are outdoors.

The public should be advised to wear repellents when they are exposed to mosquitoes as soon as an impending disease outbreak is perceived and throughout the period of risk. They should be advised to change their activities and schedules, where possible, to avoid the dawn and dusk periods when mosquitoes are generally most active, particularly in those areas where mosquitoes are very abundant. Persons who are occupationally most at risk (e.g. farmers) or most at risk because of their age (e.g. infants, the elderly) should take special precautions to avoid bites.

\section{b. Media Option}

Information can be provided to the public through the news media and through paid and/or unpaid public service announcements. Usually, a combination of approaches is taken because the news media tends to develop sensational feature stories on victims of the disease and the vector control operations rather than to issue simple, precautionary advice from health officials on such things as using mosquito repellents.

\section{Public Service Announcements}

During past health emergencies, such announcements have been published at government expense in daily newspapers to ensure that the public is given concise, accurate information on the disease and its control. Caught up in the popular issues of chemical safety, environmental concerns, etc., the news media may not be interested in or understand the importance of providing such basic information to the public.

Faced with this possibility, public information officers should prepare a series of such announcements in advance so that they can be issued quickly when the need arises.

\section{Press Releases}

Press releases contain the information that the issuer wishes the news media to pass on to the public, hopefully unchanged. But, to the news media, such releases are only possible story ideas which may be worth investigating further through personal interviews. Rarely, if ever, are press releases passed on to the public unaltered. Often, the stories take bizarre twists and turns leaving the issuer wishing that the press release had never been issued. 
Nevertheless, press releases are a tradition that continues. One can only attempt to make the most of the situation by anticipating where the press release may lead the reporter and being ready to deal with each direction the reporter may take. This means having the factual information and/or information sources available to respond to reporters questions. Ideally, the information sources will be consistent and confirm any information that has been provided in the press release. However, the more people that are involved, the greater the chance that some of these people will contradict each other, even professionals who are supposedly working towards the same goal.

\section{News Conferences}

News conferences are often the forum of choice for persons issuing information to the public through the news media. Typically, the news conference is held in a room which has been set-up to facilitate, physically, the work of the both the issuer and the reporters. The format is simple. Someone, usually a public information officer or administrative assistant, introduces the speaker and says a statement will be issued, after which the speaker will respond to the reporter's questions. A written copy of the statement is usually issued at the same time.

The speaker gives his statement and the reporters ask their questions. Sometimes, statements will be delivered by the person in charge but he answers only questions of a general or policy nature, deferring responses to questions of a technical nature to selected experts (e.g. a health official, an entomologist, a veterinarian) sharing the stage. Other times, there may be a series of government speakers and statements issued, each speaker fielding questions on their area of expertise after delivering a statement or all of the statements.

\section{c. Resource Materials}

Reference material is essential during a health emergency, particularly during an outbreak of mosquito-borne encephalitis. Information on relevant topics should be available for the public information program. Much of the needed information is contained in the various sections of this Manual and additional material may be obtained from experts in the various fields if gaps are perceived or more detail is required on specific topics.

Health officials of many different jurisdictions, after experiencing a mosquito or other arthropod-borne disease outbreak, have seen the merit in preparing audio-visual materials to describe the disease, the vectors and disease prevention and control. A modern classic example is the wealth of such materials prepared on tick-borne Lyme disease. Further, they have seen the merit of preserving every scrap of information they can obtain that documents an encephalitis outbreak, their unofficial information collections records rivalling any archival record. Such resource materials range from informative notes 
comprehensive film documentaries.

Persons involved in the public information program would be welladvised to find out who was involved during past health emergencies. Who was the epidemiologist, entomologist, veterinarian, medical doctor, archivist, freelance writer, environmentalist? etc. Equally important, one should know if those key players were for or against past emergency measures, if only to develop a list of resource persons.

The resource materials, whether they be scientific publications, technical manuals, program reports, posters, videos, public service announcements, or any other form of documentation, can be grouped under the following headings.

\section{Diseases}

Resource information on SLE and WEE should include historical accounts of past outbreaks; descriptions of the viruses and the symptoms of disease in humans; the distribution and incidence of the diseases in Canada and other jurisdictions; the prevalence of viral antibodies in the general population; the age groups and occupational types most at risk; fatality rates; the seasonal history of the diseases; clinical features; diagnosis; and the known and speculative parts of the transmission cycles.

\section{Vectors}

Information on the vectors of SLE and WEE should include descriptions of their life cycles; geographical distribution and seasonal abundance; larval breeding sites; blood-feeding requirements and sources of blood; blood-source seeking behaviour; flight activity periods; dispersal range; day-time resting sites; effect of weather on vector activity, abundance and virus transmission; methods and materials used to monitor larval and adult vector populations.

\section{Control Methods}

Reference material under this topic might be grouped under such headings as vaccination of horses; mosquito larviciding methods, materials, and equipment; mosquito adulticiding methods, materials and equipment; reduction of larval mosquito breeding sites; biological control of mosquitoes.

\section{Insecticides}

Information on the mosquito larvicides and adulticides which may be used for disease vector control can be assembled quickly if requested from the right people. For each insecticide involved, technical information might be grouped under such headings as toxicology, environmental impact; use restrictions; precautions; mode of action against mosquitoes; efficacy; residual activity; emergency procedures and contacts; suppliers; unit costs.

\section{Personal Protection}


Useful information on personal protection might be grouped under such headings as repellents; repellent-impregnated clothing; protective clothing; screened doors and windows; avoidance of mosquito bites. Each of these headings, in turn, might have a series of sub-headings.

For example, under repellents, information could be grouped on the relative effectiveness of the various repellents available; how mosquito repellents work; the pros and cons of wearing mosquito repellents; who should and who should not wear repellents; local sources, stocks and costs of repellents.

\section{Posters}

A series of posters, depicting symptoms of the disease, the transmission cycle, horse vaccination, the mosquito life cycle, and methods of mosquito control, would be useful for display in doctors' or veterinarians' offices, schools, and selected public buildings. Such a series of posters was developed after a recent outbreak of WEE in Mani toba.

\section{Videos}

A documentary videotape on dealing with a outbreak of encephalitis would also be useful. The media would be able to select portions for incorporation into news reports, saving them time and effort in obtaining difficult to obtain footage. By itself, it might be excellent teaching material for public education in schools. Again, such a video was prepared for Manitoba Health after a recent outbreak and copies may be available.

\section{Films}

Films have been prepared on encephalitis control in the U.S.A. However, these films may not be applicable to Canadian conditions and are now out-of-date, in terms of the insecticides available and the equipment used in mosquito control.

\section{Advertisements}

Paid, public service announcements were part of the 1981 WEE outbreak in Manitoba. Copies of these should be available in provincial archives.

\section{Media/Public/Private Archives}

Whenever the news media develops a major news item, it reviews its historical records for archival reference and resource material. Such reviews quickly allow a reporter to formulate an overview of the issues, develop a list of experts (often adversaries) to contact for information and outline possible approaches to the story assignment. 0ld documentary photographs and video-tapes are a source for new, supporting visual elements of the story.

Persons responsible for the government's public information program also would find such material very helpful in assembling a comprehensive 
public information package. It would certainly be worthwhile to review copies of any such material that might be on file from previous health emergencies. Knowing what the media knows, soon will know, or soon will want to know enables their questions to be answered with confidence, consistency and accuracy. Also, knowing what visual features the news media will likely want to update (e.g. mosquito biting victim, veterinarian vaccinating a horse, sick horses, Government Cabinet meeting in progress, spray planes being loaded, spraying in progress) often enables these requests to be anticipated and arranged at convenient times rather than endured while surveillance and control officials are under greater pressure.

\section{d. Specific Needs}

Each target audience has special information needs. Some examples are given below.

Chemically-sensitive persons

Such individuals will want more detailed information on the insecticides used and repellents recommended and on the schedule of spray operations over their community. They will also want to know what they can do to minimize their exposure to the insecticide.

\section{Doctors}

Medical doctors may require more detailed information on the symptoms and diagnosis of the disease, the procedures to follow for procesing blood samples, and the treatment that should be followed.

Mothers with infants

Special advice should be provided to expectant mothers and mothers with infants. Such topics include personal protection without repellents and what symptoms of the disease in infants will require attention.

\section{Horse Owners}

A horse owner, whether caring for the family pet or a thoroughbred racer, will want to know what has to be done to protect the horse from mosquito bites and the disease.

\section{Beekeepers}

Beekeepers will want to know the exact boundaries of spray blocks, the times of spray applications, what measures they can take to protect their bees from exposure to the insecticide sprayed, and what they may have to do to support any claims for bee kill or lost honey production they may make.

\section{Farmers}

Farm families, outside sprayed areas, will need special attention, emphasizing personal protection from mosquito bites and source reduction 
on their farm property.

Veterinarians

Veterinarians will need information on the disease in horses, timing of vaccinations, processing blood samples, and reporting and treating suspected horse cases.

Elected Officials

Elected officials, particularly mayors and reeves, will need to know what the risks of disease are to their specific communities and what, if any, plans, are being made to include their municipalities in any emergency spray program. The timely supply of information is critical for their support of the program at a local level. 
PART II 


\section{SURVEILLANCE PROGRAM}

a. Protocols for Arbovirus Surveillance

A Mode1: Western Equine Encephalitis

Protocols for western equine encephalitis surveillance are presented as a model for arbovirus surveillance. These protocols are the absolute minimum essential to provide an indication of risk. Additional surveillance procedures (see SURVEILLANCE, 4d) which re-enforce and expand the annual database for assessment of risk and provide a mechanism for improving the database are recommended WEE surveillance program developed in Manitoba remains the most detailed system of surveillance carried out in Canada.

Co-ordination of Program

The program should be supervised by a designated Coordinator, who is Chair of the Surveillance Committee, and who is responsible for reporting to senior administration in the Ministry/Department of Health. The Chair should also be given the responsibility of coordinating the funding authorized for arbovirus surveillance.

The Committee is responsible for carrying out the surveillance program, and should, through the Chair, report to and be accountable to the Ministry/Department of Health. The terms of reference of the surveillance committee should be well-defined and restricted to an advisory role.

b. Resource Requirements for Surveillance Program

Stable Funding

\section{Staffing}

co-ordinator

permanent staff of program (areas of expertise must include virology, public health (infectious diseases, epidemiology), medical entomology, veterinary health (horses, poultry), meteorology, administration).

seasonal staff

office staff

expert consultants (depending on expertise of permanent staff)

\section{Equipment}

laboratory facilities for virological work, laboratory facilities for mosquito handling, veterinary facilities for equine diagnosis and equine and chicken autopsies, vehicles for serum collection (including at least 1 truck), flock cages, mosquito traps, microscopes, cold tables, temperature recorders, rain gauges, wind recorders, chickens. 


\section{Supplies}

virological supplies, mice, reagents, picnic coolers (1+ per trap site), dry ice; entomological supplies, collection apparatus (aspirators), forceps, mosquito cages, cryovials, sealing tape, labels, repellent; sentinel flock supplies, repair kit, chicken feed (complete grower ration), medication, and water supply, capillary tubes; veterinary supplies, for collecting horse serum samples, autopsy samples; office supplies, including report preparation and distribution; travel support for field workers.

\section{c. Components of Surveillance Program}

1. Weather

Weather data should be monitored over several points across the province, and interpreted by a meteorologist. Atmospheric Services of Environment Canada provide these services on a userfee basis.

\section{Pre-season Assessment}

The following conditions should be reviewed and the implications on the current season assessed: soil saturation levels and late season rainfall of previous year, snowfall, mean average weekly temperatures for winter months, late winter/early spring mean average weekly temperatures, and anticipated rate of snowmelt.

\section{Seasonal Monitoring}

Weekly weather forecasts and updates should be prepared as data available on soil saturation levels. Retrospective weather reports on previous week should be prepared.

\section{Reporting Techniques}

Retrospective and forecast reports on conditions should be presented in a standardised format including weather map(s) covering surveillance area, and indicating weather stations/data collection sites. 'A narrative summary of weather conditions should be provided in a consistent weekly format, and be easily interpreted by people without scientific training or experience.

\section{i1. Mosquito Monitoring}

Flock traps: A detailed description of the flock trap is given by Wong et al. (1976), Wong and Neufeld (1982). Prior to each spring, all traps should be checked, and repaired as necessary. Flock trap cages are subject to vandalism as well as damage by animals attracted to the birds. Careful attention should be made to mesh screening, and attachment of mosquito trapping component. To protect traps from exposure to the elements, repaint, as necessary. Each trap/trap site should be 
equipped with continuous max/min temperature recorders, rain gauges, wind recorders.

Traps sites should be carefully selected, using the following criteria: site not too exposed but with reasonable vegetation providing shelter, proximity to breeding sites and preferred blood sources, history of surveillance or monitoring of mosquito populations, proximity to human population centres, safety from vandalsim, ease of access for surveillance crew, ease of maintenance (i.e., watering and feeding flock).

\section{Trapping Frequency}

Daily whenever possible; minimum of. weekly (unless populations increases above non-outbreak levels) and biweekly during critical periods (mid June to mid July). Mosquitoes should be sorted into five groups: $C x$. tarsalis, $C x$. restuans, $C s$. inornata, Ae. vexans, and 'others' and counted (see 3 below).

A minimum of five traps, located in different areas over jurisdiction; 12 to 15 traps are recommended. No additional traps should be set up in an emergency unless in areas where there is prior knowledge of WEE activity.

Reporting Techniques

Trap catches should be reported weekly and in a consistent format which includes three parts.

1) tabulation for each trap site per trap/night (per trap/week for summaries): the total number of female mosquitoes caught, total number of $C x$. tarsalis caught, and percentage of $C_{x}$. tarsalis of total number caught.

2) graphic comparison both with average catch for non-outbreak years and average catch for outbreak years for the same collection period.

3) a narrative presentation of trap results, interpreting counts to ambient conditions of temperature, precipitation, wind, and any other pertinent information (e.g., change to local environment). The narrative should also follow a consistent format, and be easily interpreted by people without scientific training or experience.

Other trapping, including livetrapping of mosquitoes for viral analysis with $\mathrm{CO}_{2}$-baited traps, and using standard New Jersey style light traps (see SURVEILLANCE 4b), is recommended. However, these trapping strategies are considered lower priority than a network of flock traps. 
1i1. Virus Activity

Mosquito Isolations

Mosquitoes should be collected daily from each flock trap by using a battery-operated aspirator. Mosquitoes removed from traps should be confined in mesh cages and transported, in coolers, promptly to the laboratory for immediate sorting and counting. Mosquitoes should be kept cool until and during counting. Mosquitoes should be retained on a cold table ( $-5 \mathrm{C}$ ) while being sorted, identified, and counted. Those mosquitoes retained for virological testing should be pooled according to species, trap location, and date of collection. Mosquitoes should be pooled and tested in the following priority: all Cx. tarsalis, all Cx. restuans, all Cs. inornata, and Ae. vexans for the remaining quota of weekly pools. Pooled mosquitoes should be live when processed, or kept frozen at temperatures $-70 \mathrm{C}$ until processing, to prevent loss of virus. (Where $-70 \mathrm{C}$ storage is not immediately available, short term storage of pooled mosquitoes, well protected from exposure to carbon dioxide, at $-40 \mathrm{C}$ in dry ice may be used, but only with risk of underestimating virus infection of mosquitoes.)

The number of pools processed weekly will vary according to trap catches, and may require the use of a weekly maximum number (quota) which depends on availability of resources. The number of pools to be processed should be consistent, annually. Pools should consist of a maximum of 50 mosquitoes per pool; and fewer for pools of Culex species, to allow more precise assessment of minimum field infection rates.

Pools should be processed using recognized virological techniques. Several techniques are available, and the system adopted may depend in part on available expertise and facilities. Processing should be made and results confirmed within four to five days of pools being submitted for analysis (five to six days post collection). Analysis by cytopathology in tissue cultures (embryo mice, or VERO, BHK-21, primary AFGMK cell cultures), with virus isolations confirmed by neutralization test (Sekla and Stackiw, 1976, 1982) is recommended. Identity of isolations should be confirmed by the National Arbovirus Reference Centre in Toronto.

Reporting techniques- results of tests should be reported weekly and in a consistent format which includes three parts: 1) tabulation of pools per date submitted, per species, per location, and including i) percentage (of total pools) of positive $C x$. tarsalis pools/date/location, $i \mathrm{i}$ ) percentage (of all $\mathrm{Cx}$. tarsalis pools) of positive $C x$. tarsalis pools/date/location, and iii) numbers of mosquitoes/pool.

2) graphic comparison of season results to date with average results of non-outbreak years and with average results of outbreak years. 
3) a narrative interpreting results and comparing results with frequency and extent of positive pools in average non-outbreak years as well as average outbreak conditions should also be provided in a consistent format.

\section{Sentinel Flocks}

Flock animals should be selected for survival under field conditions and handling. In Manitoba, the preferred sentinel host is a cross between Rhode Island Red and White broiler chickens (raised indoors), five weeks old at the beginning of surveillance. Flocks, consisting of fifteen birds each, should be set up, retained in flock trap cages (Wong et al. 1976, Wong and Neufeld 1982). Birds should be provided with a constant supply of fresh water, food and medication (Wong and Neufeld 1982).

Birds should be tagged individually for identification. They should be established as free from prior exposure by a preexposure bleeding. Subsequent bleedings should be made biweekly unless indicators suggest above normal (i.e., above non outbreak) conditions, at which time birds should be bled weekly. All birds should be bled from the deep ulna vein, and the blood allowed to clot. Positive birds should be replaced with pre-bled birds (acquired at the same time as the sentinel birds, and maintained as potential replacement birds, as needed), calculations of rates of seroconversion should exclude replacement birds until they have been exposed for two weeks.

Serological samples should be processed using recognized virological techniques. Haemaggluttination-inhibition (HI) and confirmation of $\mathrm{HI}$ positive samples with complement fixation (CF) (Sekla and Stackiw 1976, 1982) is recommended. An HI titre of 1:32 should be considered evidence of seroconversion. Positive samples should be confirmed by the Animal Diseases Research Institute (Agriculture Canada), in Ottawa.

Reporting techniques - results of tests should be reported weekly and in a consistent format which includes three parts: 1) tabulation of rates of seroconversion per date and per location, including number of birds infected/site, number of birds surviving per site, and number of replacement birds placed per site.

2) graphic comparison of season results to date with average results of non-outbreak years and with average results of outbreak years.

3) a narrative interpreting results and comparing results with frequency and extent of seroconversion rates in average nonoutbreak years as well as average outbreak conditions should also be provided in a consistent format. Interpretation of serconversion data should be made with account of mosquito monitoring data, virus isolation rates, and bird mortality. 
1v. Equine Cases

An inventory of veterinarians should be compiled and updated annually. Advisories should be sent out to all practising veterinarians informing them of the surveillance program, describing pattern of equine infections and listing symptoms of infection. In conjunction with the advisory, veterinarians should be requested to submit two blood samples from horses suspected of being infected to the provincial veterinary laboratory. The first sample (acute sample) should be taken at onset of symptoms; and the second, needed for confirmation of diagnosis, after two to four weeks. Sample submissions should include data on age and vaccination history of sick animals, and data on residency or travel in the area of possible infection. Veterinarians should also report frequency of horse vaccinations in their practice.

Confirmation of equine infection should be carried out by HI test, with positives (of acute and subsequent samples) confirmed by complement fixation (CF) test, or neutralization tests for virus isolation (Sekla and Stackiw 1976, 1982; Wong and Neufeld 1982).

Confirmation of a diagnosis of WEE infection in horses is required, and should be based on the following criteria:

i) paired samples (acute+subsequent) must show a 4 fold or greater increase in antibody titre (by HI or CF tests).

ii) single samples (if no second sample is available) must show an HI titre of 1:64 in young (less than one year) animals with no history of vaccination and with CNS symptoms; an antibody titre of 1:256 in horses of any age and immunization status; an antibody titre of 1:1024 in older horses with a known history of vaccinations.

iii) Infection of horses which died without blood samples being collected can be confirmed by examination of brain tissue for lesions (if tests show animal is negative for rabies infection), or by presence of WEE virus confirmed by neutralization test.

Positive samples should be confirmed by the Animal Diseases Research Institute (Agriculture Canada), in Ottawa.

\section{Reporting Techniques}

Incidence of possible equine infections and results of tests should be reported weekly and in a consistent format which includes three parts:

1) tabulation of numbers of submission of samples of suspected infections/week, of numbers of confirmed cases submitted(by week of submission)/week, numbers of deaths/week.

2) graphic comparison of season results to date with average results of non-outbreak years and with average results of outbreak years.

3) a narrative interpreting results and comparing results with 
frequency of suspected and confirmed cases in average non-outbreak years as well as average outbreak conditions should also be provided in a consistent format. Interpretation should include information on location and age of horse cases, status of suspected cases, and an account of exposure period, weather, mosquito monitoring data, virus isolation rates, and bird mortality.

\section{v. Human Cases}

Monitoring of human infections should not be part of predictive function of surveillance program, but should be carried out as part of the assessment of severity of an outbreak.

Tests for WEE infection in patients are carried out at request of a physician. Blood sera should be analyzed by recognized techniques, which include $\mathrm{HI}$ and $\mathrm{CF}$ tests for screening, neutralization tests, and, if infection is recent, immunoglobulin $M$ (IgM) antibody determinations (Sekla and Stackiw 1982).

Reports of suspect and confirmed cases should be reported immediately, with details of date of onset of symptoms, age, activity, residence/location of (suspected) infection.

vi. Contributions and Activities from Other Jurisdictions Contributions from other jurisdictions are only useful if there is a formal agreement to contribute and if procedures, scheduling, and techniques are compatible with the surveillance program. Regular weekly contact with a supervisory personnel in other jurisdictions should be made by co-ordinator of the program.

Weekly contact should also provide information on activities of other jurisdictions in mosquito control or in other insect control which may impact on surveillance data.

Collaboration with Neighbouring Jurisdictions

Information from neighbouring jurisdictions provides valuable information for the surveillance committee by assisting assessment of likelihood of virus activity.

Reporting Techniques

The co-ordinator should make regular, weekly (or less, as risk assessment requires) contact with supervisory contact person on a pre-arranged schedule. Exchange of information should be made in a consistent format.

\section{d. Approach}

Surveillance should be started early in the spring, and continue until fall. Staff should be hired, rather than volunteer help used, because of the requirement for reliability of regular trap collection which is not met when volunteers are used. 
Account must be made of weekends, holidays, staff time off for field staff.

A system of communicating information to the Minister of Health, the media, and the public must be planned well in advance. This should be coordinated through the Chair, and regular reporting times should be scheduled, and rigidly followed.

Activity Schedule

Setting up program: January-February

Review areas of activity, procedures, lines of authority.

Review criteria of risk; review objectives, annual

procedures; evaluate any innovations to program

Arrange hiring of seasonal help

Contact neighbouring jurisdictions; plan group workshop/joint meeting

Schedule meetings on weekly basis

Establish system of communications

Make inventory of equipment, supplies

March - April

Train seasonal help

Purchase equipment and supplies; repairs equipment as necessary

April

Prepare flocks and set up flock traps

Prepare recording procedures, record sheets, maps of trap locations, record logs

Arrange computer programming for report preparation

Weekly Program

Regular weekly update of surveillance status.

Monthly program

Plan reporting schedule

Season wrap-up

Prepare annual/seasonal reports and distribute. 


\section{CONTINGENCY PLANNING FOR IMPLEMENTATION OF EMERGENCY VECTOR CONTROL}

This section is a description of the general approach to aerial ULV applications, resource requirements, planning procedures, jurisdictions, operating procedures and reporting activities involved in emergency vector control during an outbreak of mosquito-borne encephalitis. Although the guidelines contained in this section are based on Manitoba's experience in dealing with outbreaks of Western Equine Encephalitis (in 1975, 1977, 1981 and 1983), the basic concepts should be applicable to the control of other mosquito-borne diseases in Canada. As policies and procedures change over time and vary from province to province, changes should be expected.

The technical information and advice given in this section are designed to assist someone who has been appointed to organize and direct an emergency spray operation, enabling a fast and organized response to the health emergency. In addition, it should help those officials and personnel who are supporting the field operation to understand what is involved in major emergency vector control.

Although this section is designed to be a detachable field reference, users should refer to the first part of this manual for a review of the more general aspects of vector suppression, in both an emergency and a non-emergency context.

\section{a. General Approach to Aerial ULV Applications}

If the situation warrants, the Minister of Health will declare a health emergency due to the threat of mosquito-borne encephalitis. This decision will set the emergency vector control program into motion. Before the decision is made, an assessment of the risk to human health and the province's capacity to minimize that risk will have been made. Included in that assessment will have been a determination of the best way to reduce the mosquito vector population and, thereby, the risk to public health.

Both ground-based and aerial vector control measures will have been reviewed. Aerial ultralow volume (ULV) application of insecticide will have been recommended as the most economical and efficient means of large-area mosquito vector control. The aerial application firm with the experience and equipment to apply the mosquito adulticide will have been contacted to determine its availability and how soon it can position itself within the province. The discussion below will assume that an experienced aerial application firm (such as Conair Aviation with a DC6 spray plane equipped with a Beecomist ULV spray system) will be hired. The supplier of the chosen insecticide also will have been contacted to determine how soon it can deliver the estimated insecticide requirements. Prices of these goods and services will 
have been discussed if not negotiated and finalized. Arrangements will have been tentatively made to secure the required support personnel, facilities, equipment and cooperation that will be required during the health emergency.

Coincident with these preparations, someone with the required knowledge and experience will be selected to direct the largearea mosquito vector control program. That person will likely be an entomologist who has managed a large mosquito abatement program, is familiar with the methods, materials and equipment used in major aerial ULV aerial applications of insecticide for mosquito control, and can draw on a network of professional contacts for advice and assistance as required during the course of the emergency operations.

In preparation for the vector control program, the director of spray operations will be affirmed and, with the emergency measures coordinator, review what resources are required to carry out the vector control program in the safest, most economical and most efficient way possible. He will assist the emergency measures coordinator in setting up the field operations base at the nearest suitable airport and begin developing detailed spray block designs and a spray schedule with the aerial applicator.

Once the field operations base has been established and the required support personnel, supplies and equipment have arrived, the operations director will oversee the actual spray operations. Progress at every step will be reported to and general direction will be received from the Deputy Minister of Health, through established channels.

After the spray operations have been completed, the operations director will assist with the dismantling of the operations base, including such things as the return of unused insecticide to the manufacturer and the disposal of contaminated materials. He may also be involved in debriefing the Minister on the aerial spray program, in a review of the emergency expenses, and in the preparation of a report on the work carried out, including recommendations for future such programs.

All of this may seem fairly simple and straight-forward. However, the description given above is only an outline of some of the key aspects of the vector control program from the operations director's point-of-view. Much more is involved. Plans for, organization of and operation of the aerial ULV applications is described in more detail below. Again, it is written from the perspective of the spray operations director.

\section{b. Resource Requirements}

Funding

Emergency funding to cover all aspects of the health emergency (including surveillance, public information, vector control, efficacy evaluation, and environmental monitoring) will 
be needed. Because the legislature will likely be in summer recess when the outbreak occurs, Cabinet will have to meet and approve the estimate expenditures.

Cost estimates for the vector control operations will have to be developed, based on the number and sizes of the spray blocks, the insecticide required for their treatment, the cost of aerial application and various equipment and supplies needed. The Director of Spray Operations will assist in the preparation of these estimates. These estimates will be considered, along with those of the various supporting programs, by Cabinet.

Operations Personnel

The Director of Spray Operations, reporting directly to the Deputy Minister of Health, initiates and carries out the required activities of spraying cities and towns at risk. He coordinates all of the activities at the Field Operations Base to ensure that aerial spraying is carried out safely, efficiently and in a professional manner. He liaises with provincial and federal officials, university researchers and other organizations that monitor and assess the efficacy and impact of spraying.

The person selected to be Director of Spray Operations ideally should have a M.Sc. or $\mathrm{Ph} . \mathrm{D}$. in medical-veterinary entomology, at least 5 years practical experience in managing a major mosquito abatement operation, and a good knowledge and understanding of the safe handling and application of insecticides.

Before spraying begins, the Director may be required to review draft contracts for aerial application and for the supply of insecticide on behalf of the province and to develop detailed cost estimates based on final agreements with the suppliers of goods and services.

With the provincial emergency measures organization (EMO) representative (usually designated as the Communications officer) and the airport duty officer, the Director of spray operations will also assist in locating and assembling the Operations Base taking into consideration such matters as a ramp area for the aircraft, an area for loading, an area for insecticide storage tankers, an area for a field office and meeting room and an area for parking vehicles and storing equipment and in determining who receives visitor passes and vehicle passes from airport security. Also, the airport fire, ramp security and police should be briefed as to what will be done at the airport and any potential problems associated with the operations.

Also, all necessary equipment and supplies (detailed below) will be requested, including office supplies, safety gear and protective clothing, spill control and clean-up materials, special maps, portable generator and lights, washroom and emergency shower, small tools, and two-way radio and telephone communi- 
cations equipment.

The aerial applicator will be responsible for the supply of all equipment, tools and supplies associated with transferring the insecticide from the storage tanks to the aircraft, for fuelling the plane and for the maintenance of the aircraft and spray systems. They will also be responsible for arranging accommodation, food services and transportation for their staff.

As soon as the cities and towns likely to be sprayed are known, the Director will acquire the necessary topographical maps for these areas. These will be used to plot the spray blocks. At least three copies of each must be prepared. The aerial applicator, the public information officer, and the lead monitoring agency will each require a copy. The spray blocks will be designed in consultation with the aerial applicator.

Typically, the spray block is square or rectangular (usually $13 \mathrm{x}$ $13.5 \mathrm{~km}$ in size), centred over the community and, includes the community and a surrounding buffer zone of about $10 \mathrm{~km}$ in all directions. From these maps, the applicator can determine the longitude-latitude coordinates necessary for programming the onboard, computerized, navigation system for each spray block.

The Director also may be required to attend various meetings of the Core Coordinating Committee, the surveillance committee, and the various monitoring organizations to brief them on the status of preparations being made for spraying and to explain the various procedures that will be followed, including the criteria for spraying and methods of communication.

Different tasks may be designated to other members of the spray team. The manufacturer's representative may be asked to oversee the provisions for storage tankers, the transfer of insecticide into them from arriving tanker trucks and out of them to the aircraft, spill control, sampling for chemical assays, calculating insecticide requirements for each flight and obtaining technical information on the insecticide for use by various agencies and individuals associated with the health emergency. The supervisor of the aerial application crew may be asked to plot spray block maps, lead media tours of the aircraft and prepare written post-flight summaries. The EMO Communications Officer may be asked to open and close the facilities daily, maintain a written operations log (noting when personnel arrive and leave the site and where they can be reached in an emergency, all deliveries, spray flight times, telephone calls, etc.) and, of course, communicate information to and from the various support groups.

During the spray operations, the Director, working at the Operations Base, will lead a spray team, comprised of himself, the supervisor of the aerial application crew (including pilot, navigator, and maintenance crew), the technical manager of the insecticide manufacturer, and the EMO officer on site. Based on biological, meteorological and operational criteria, the spray 
team will decide which of the possible spray blocks should be sprayed at any given opportunity. The basis of all such decisions should be noted in the written log.

The Director, as final decision-maker, will issue a workorder for each flight and it will proceed as planned, barring mechanical problems or last-minute changes in weather conditions and he will have this information passed on, through pre-arranged channels, to everyone who must be advised.

Following each flight, the Director will be briefed by the aerial application staff on the spray operation, including any problems encountered, their observations on wind conditions and insecticide drift, etc.

When the spray operations have been completed, the Director will oversee the disassembly of the Operations Base, including the applicator's equipment, storage tankers, field office and meeting room, etc. An inventory of all remaining equipment, tools and supplies should be made. Also, he should assemble all the paperwork that was generated during the operations (including the log, work-orders, delivery slips, maps of spray blocks, etc.) for delivery to the EMO. This information may be vital for the province to deal with possible future damage claims. If the Director is required to prepare a written report on the operations to the Deputy Minister of Health, he should ensure he has a copy of all the required documentation should be ensured.

Equipment

A variety of equipment is needed at the Operations Base for routine, occasional and emergency use. The requirements may vary from operation to operation, depending upon the equipment provided by the aerial applicator and the EMO. Some of items found helpful, if not essential during past operations are listed below.

\section{Field office}

The provincial EMO may have a fully-equipped, mobile field office available for use. If not, a large construction trailer can be rented and equipped with tables, chairs, filing cabinet, bookcase, waste-paper baskets, desk trays, clock, lamps, bulletin boards, pencil sharpener, calculators, typewriter, photocopier, chalk-board, tape-recorder, multiple telephone lines, portable VHF transceiver, public address system, pagers, fax machine, and other equipment.

Meeting/Lunch Room

A second rented construction trailer should be equipped with tables, chairs, coffee machine and supplies, cot, bulletin board, ashtrays, clock, and any other equipment that might be useful in a combined meeting-lunchroom for the operations personnel that are 
working from before dawn until well after sunset every day for 24 consecutive weeks.

\section{Protective Clothing and Facilities}

Many items are necessary for safely transferring insecticide from arriving transport tankers to the storage tankers and from these to the aircraft and for personal hygiene. These items include fire extinguishers, first aid kit, rubber-coated apron, rubber boots, coveralls, ear-plugs, face-shield, hard-hats, rubber gloves, goggles, respirators and cartridges, rain-suits, eye-wash station, emergency shower, portable barricades, portable toilet, pesticide spill absorption material, and scoop shovels.

\section{Insecticide Storage/Loading Area}

A variety of tools and equipment are needed in the storage/loading area. These include small tools (including mechanic's tool-box, metric and imperial socket wrench sets, hammer, pliers, hack-saw, vice-grips, wire-cutters, tape-measure, screw-driver set, drum plug wrench); stop-watch; polypropylene rope; garbage cans; empty drums (with tops removed); padlocks; heavy-duty extension cords (various lengths); mechanic's light; portable outdoor lighting; insecticide transfer pumps, hoses, couplings, filter, cartridges, and spare parts (usually provided by the aerial applicator); a rented, 40,000 L capacity, compartmentalized, tanker-trailer unit for the bulk storage of insecticide, kept for the duration, with wooden-crib supports.

\section{Supplies}

Many different materials are used during the emergency spray operations. These are categorized below.

\section{Field office}

Office supplies, including pencils, pens, chalk, markers, high-lighters, rulers, yardsticks, dividers, compass, erasers, rubber bands, paper-clips, transparent tape, masking tape, electrician's tape, file folders, legal and $22 \times 28 \mathrm{~cm}$ envelopes, carbon paper, archive boxes, 2 -way memos, typewriter correction fluid, $22 \times 28 \mathrm{~cm}$ paper, note-pads, clip-boards, 3-ring punch, 3ring binders, staplers, scissors, utility knife, date stamp, ink pad, calenders, thumb tacks, message pads, long-distance record pads; telephone directories (city, province, provincial government); dictionary; maps, including topographical maps, provincial highway maps, and city street maps; maintenance supplies, including broom and dustpan, cleaning rags, paper towels, window cleaner, and garbage bags; thermos water-jugs; flash-lights and spare batteries. 


\section{Insecticide}

Insecticide should be delivered by tanker truck directly from the manufacturer to the Operations Base. The first trailer units, with a combined storage capacity of at least 40,000 L and kept as storage tanks for receiving future shipments, must be properly sited and supported. Sufficient insecticide should be ordered to treat the spray blocks for all known communities at risk. Additional insecticide should be ordered as soon as new communities are added to the list.

Inventories of Personnel, Equipment and Supplies

Much time will be saved if resource lists are up-dated annually by the EMO or some other designated agency for operations personnel. The most important such listings are given below.

Suppliers of Services, Materials and Equipment (supplier's name, address, telephone numbers [working and non-working hours], fax numbers, type of equipment/supplies/services, quantity on hand or time required to produce/obtain and supply).

Resource Personnel in Government, Universities, and Business (including the names, addresses, and telephone numbers of professionals, technical experts, operations personnel, etc.).

\section{c. Planning and Scheduling}

Contingency plans may have been prepared for a provincial health emergency. If so, various committees probably have been established, if only one paper, to deal with the emergency. An emergency involving an outbreak of mosquito-borne encephalitis may or may not fit into an existing general health emergency plan. In any case, a coordinating committee, composed of the relevant departments will lead and direct the various surveillance and vector control activities.

In the case of a mosquito-borne encephalitis outbreak, this lead committee (which may be called the Core Coordinating Committee or Task Force or something else) will probably consist of the Deputy Ministers and Ministers of Health, Emergency Measures, Environment and Agriculture. Key professional staff will report to them (occasionally or regularly, directly or through their Deputy Minister) during the emergency. These staff may include the chairpersons of the surveillance and monitoring committees, the provincial epidemiologist, the director of the provincial diagnostic laboratory, the provincial entomologist, the provincial veterinarian, the public information coordinator, the director of purchasing, the EMO coordinator, the provincial solicitor, etc.

Regardless of the reporting system, this lead committee will require complete up-to-date information and recommendations each time it meets. It will be under pressure to make decisions which may or may not be popular to the public, the media, environmental 
groups, professionals, etc., which may have major health, economic, social, and political consequences. Thus, this lead committee depends on the best professional, scientific and practical advice that is available to it.

At the beginning, the lead committee will be deciding on who will be responsible for doing what and when it should be available. Appointments to key temporary positions will be made. Authority will be given to acquire vector control equipment and supplies, escalate surveillance activities and diagnostic services, organize monitoring programs, develop an emergency public information program, second staff, call back key personnel from vacation leaves, etc. Obviously, during the first few days at least, the activities of this committee and the people supporting may appear chaotic. The success of the lead committee will be entirely dependent on how well the members of the committee and the people working with them interact. Much will depend on the leadership abilities of the Minister of Health and the Deputy Minister.

Based on the information and advice given to them, the lead committee will oversee the emergency surveillance, public information and vector control programs. They will decide which communities should be sprayed and direct the operations group to do so as quickly and safely as possible. The spray operations group will be expected to develop a tentative spray schedule, based on the lead committee's priorities, usually the largest and closest population centre first and the smallest and most distant last if the incidences of disease are distributed throughout a given region of the province.

Changes in scheduling the individual spray blocks will be unavoidable if the spray operations are to take advantage of any opportunities that local weather conditions permit. One should not be waiting around to spray one town because of poor weather conditions if spraying conditions are good over another town. Changes in plans may make the work of the support groups difficult, if not impossible, at times but the key concern should be the protection of as many people as possible as soon as possible.

Additions to the initial list of towns and cities at risk should be expected, especially if the emergency was recognized relatively early. Additional supplies will have to be acquired, spray block maps drawn, and so on.

\section{d. Legislation and Jurisdiction}

From a legal and jurisdictional point-of-view, dealing with an outbreak of mosquito-borne encephalitis within a given province is that province's responsibility. If it does not have the resources to cope with the problem, it can call upon the federal government for assistance. Usually, some of the resources are 
available and those that are not can be obtained fairly readily from other jurisdictions through existing provincial and federal cooperative agreements. Depending upon the scope of the emergency and the costs incurred, the province may be eligible for federal aid.

From a vector control point-of-view, several legal matters must be considered. These include the official declaration of a provincial health emergency (and, later, its termination), the waiver of those sections of any provincial Acts or regulations that might prevent the province from implementing a large-area, aerial application of insecticide for vector control, adherence to the federal Pest Control Products Act and its regulations, respect for the International Migratory Birds Convention, the waiver of federal aviation regulations governing low-level flights over populated areas and within air traffic control zones, the successful negotiation of contracts with major suppliers of goods and services, and the approval of funds for emergency expenditures. The lead committee, Spray Operations Director, EMO Coordinator and provincial solicitors will have to ensure that all these matters are finalized before spraying begins.

The declaration of a health emergency enables all the other legal matters to be considered. A formal emergency declaration is usually made by the Minister of Health on the advice of provincial health officials and the disease surveillance committee. The basis of such a recommendation to the Minister is a combination of known virus and vector activity and suspected or confirmed cases of the disease with an expectation that the health situation will deteriorate unless there is intervention. This declaration will reinforce the vector control authority of the Minister and serve to activate various support and assistance agreements with other government departments (provincial and federal).

After considering the findings and recommendations of the surveillance committee, the first decision of the Minister is whether or not the situation requires a formal declaration. If so, the Minister will advise and consult with other Ministers of Cabinet, including the Attorney-General and the Minister

responsible for Emergency Measures. The necessary papers will be prepared for the Minister to formally declare a health emergency.

Similarly, when the surveillance committee and/or provincial epidemiologist consider the health emergency to have passed, the Minister will be advised to formally declare the health emergency to be terminated. Usually, this will occur soon after the vector control program has been completed and no further human cases have been detected.

\section{e. Procedural Guidelines}

Several key operational procedures, which have been developed during past spray operations and may serve as guidelines for 
future emergency vector control operations, are outlined below.

\section{Estimating Insecticide Requirements}

Knowing the names of the cities and towns that will be sprayed, topographical maps can be used to draw spray blocks encompassing each of these communities. Most of the population centres will be small. A $13 \times 13.5 \mathrm{~km}$ spray block will protect these centres. The area of such a block is 17,550 hectares $(43,348$ acres $)$.

Some blocks (e.g. those of larger cities or those with unusual terrain) may be rectangular or irregular in shape. The important point is to ensure that an adequate buffer zone surrounds the community.

Knowing the insecticide and the approved application rate, the quantity of insecticide required can be calculated. Adding up the insecticide requirements for all of the spray blocks and allowing an extra 500 litres for insecticide remaining in transfer pumps, hoses and filters and the bottom of spray tanks, the insecticide requirements can be estimated.

For example, if the total area of the spray blocks was 500,000 hectares and the insecticide was applied at $0.4385 \mathrm{~L} /$ hectare, one would need $219,250 \mathrm{~L}$ for the treatments.

\section{Storage of Insecticide}

Bulk insecticide will be shipped by truck, directly from the manufacturer's formulation plant to the Operations Base, in tank trailers. Tankers are required to store the arriving insecticide. From past experience a storage tank capacity of at least 40,000 L is essential. One large or 2 smaller tank trailers meet this requirement. Three spray blocks could be treated with this amount of stored product. Usually, the first one or 2 tankers to arrive are used for this purpose until they can be rented locally. While the insecticide in these storage tanks is being used in the vector control program, additional supplies will be en route.

Ideally, such tanks should have both ends sloped to provide fast drainage, a 4-compartment manifold with a $7.5 \mathrm{~cm}$ gate valve outlet allowing curbside discharge and mechanical emergency valves and operator in the cabinet.

Such storage tankers must be situated adjacent to the aircraft's parking position for easy loading. They must be properly positioned and then reinforced with heavy timbers or supported by a multi-wheeled trailer dolly for safety reasons.

Transferring Insecticide from Delivery to Storage Tanker

The driver of the delivery truck will usually handle the transfer of material from his tanker to the storage tanker with the assistance of operations personnel. 
Before the transfer begins, someone knowledgeable in the safe operation of tanker trailers should be responsible for ensuring the following: inspecting the storage tanker to ensure that it has sufficient room available to accept the full load being delivered; blocking the wheels of the tanker; grounding the tanker; ensuring the transfer hoses are in good repair, sufficient lengths of hose are available, and all connections are secure; ensuring the tanker vent is present or the lid is open and that the bottom valve is closed; ensuring that everyone involved is wearing all necessary protective clothing and that the spill control tools and supplies are on hand; ensuring, if filling is being done through the open top Iid, that the insecticide transfer hose has been securely fastened in place using heavy wire.

A delivery/transfer record form should be completed, detailing the shipment number, the supplier, the carrier's name, the driver's name, the shipment tanker number, the storage tanker number, the date and time transfer occurred, the weigh bill number, the personnel involved in the transfer, and a description of any spills and actions taken to clean-up the spills.

Transferring Insecticide from Tanker to Aircraft

The aerial application crew will locate and assemble the transfer pumps, liquid meter, filter unit, hoses, and related gear needed for loading. Insecticide is transferred through $7.5 \mathrm{~cm}$ reinforced hose equipped with quick-couplers. Two pumps with a capacity of $900 \mathrm{~L}$ per minute, are connected in tandem to the tanker. Next, a metering device, calibrated for the insecticide to be used, is linked by hose to the pumps. Then, an upright filter canister is employed to filter out any particulate contamination. Lastly, a long hose is attached to the intake valve of the aircraft.

Before loading the aircraft, ensure the vents or top lid is open on the storage tanker to prevent the tanker walls from collapsing under vacuum. Before opening the emergency valves, ensure all hoses are properly connected at both ends. In case of emergency, the emergency valves may be closed by operating the remote controller located at the front end of the tanker.

Four persons are needed for loading. One person for relaying signals from the ground crew to the aerial crew, one person onboard for monitoring liquid levels in the on-board spray tanks, and two people for opening and closing valves, starting and stopping pumps, monitoring the meter and disconnecting and moving hoses from the aircraft.

In this fashion, sufficient insecticide for one small spray block (about $9000 \mathrm{~L}$ ) can be pumped on-board in 11 minutes.

Preparation of Spray Block Maps

often, several topographical maps must be pieced together to 
have a sheet on which the spray block can be drawn. The National Topographical Series is used at a scale of 1:50,000 (1 cm $=0.25$ $\mathrm{km}$ ). In Manitoba, these maps are available from the Maps Sales office of Manitoba Natural Resources. Features important to the aerial application including population centres, roads, forests, swamps, water bodies, reservoirs, contour lines, transmission lines, rail-roads, and towersare all present on the map. Because 3 finished sets of spray block maps are needed, the maps are best prepared in a room with adequate table space.

The 3 sets will be used by the pilots (these are considered the originals for legal purposes), the EMO (including the public information officer) and monitoring/assessment agencies. The originals will have annotations made on them pertaining to the aerial application, including flight times and date, any observations made by the pilots, start and finish points, the flight line and directional sequence followed, etc.). They should be provided to the EMO after the operations are complete.

If original maps from previous emergency spray programs are available when the current emergency is declared, they should be provided to the Spray Operations Director to facilitate the preparation of new maps.

A legend should be given for each spray block map including name of the community, date and time sprayed, load number, waypoint coordinates and line numbers. The map itself will have the flight lines (drawn in a North-South direction) that will be followed by the spray plane. Depending on wind direction when the plane arrives over the spray block, spraying may start on either side of the spray block. Usually, the navigation system is set to 2 points on the east side of the spray block (the NE and SE ends of the first flight line, usually marked ' 8 ' and ' 7 ', respectively). These 2 points are called 'waypoints' and are used to determine precise latitude-longitude coordinates for setting the computerized navigation system (i.e. an inertial navigation system such as the Litton LTN-51 or a Loran system such as the Texas Instruments TI 9100).

A typical spray block for a small rural town is $13 \times 13.5 \mathrm{~km}$ allowing about a $6.5 \mathrm{~km}$ buffer zone around the community. This design minimizes mosquito movement into the community after spraying takes place and allows for some displacement of the actual block sprayed due to droplet drift with wind. Larger cities will usually require an irregularly-shaped design, reflecting their uneven growth in different directions.

The typical $13 \times 13.5 \mathrm{~km}$ block provides for $17 \mathrm{~N}-\mathrm{S}$ flight lines $13 \mathrm{~km}$ in length and $0.8 \mathrm{~km}$ apart. The $\mathrm{N}-\mathrm{S}$ line orientation provides for a cross-wind component necessary for spray droplet dispersal and drift. Thus, spray flights can never be flown when the winds are coming from due $N$ or due $S$. (This was not a serious operational problem in August during 1975, 1977, 1981, or 1983, 
in Manitoba, because the wind was not directly $\mathrm{N}$ nor directly $\mathrm{S}$ for very long.)

General Record-Keeping

Difficult as it is to recognize and make notes on every significant meeting that is attended, activity that takes place, event that occurs, and telephone call that is made at the Operations Base, it must be done. The key personnel (including the Spray Operations Director, manufacturer's representative, aerial crew supervisor, and EMO Communications officer) should each maintain chronological records of everything they do and discuss with others. For everyone's benefit, these people should meet daily to review what has happened in the last 24 hours and what plans have been made for the remainder of the spray operations. Each person can update the others on significant points for them to record. Their records will prove invaluable to themselves and their organizations after the emergency program is finished for financial, legal, and technical reasons.

Every receipt, delivery slip, work-order, expense claim, requisition, purchase order, invoice, memo, letter, report, spray block map, etc. must be properly noted, organized and provided to the appropriate support group. Such details are usually handled by the Communications officer.

\section{f. Reporting Procedures}

Each province may have its own reporting procedures, depending upon how it has organized itself to respond to an outbreak of mosquito-borne encephalitis. Even approved contingency plans can be expected to change, depending upon the policies of the government responsible, the senior administration, the social and economic climate. However, reporting lines and procedures should be made known to everyone as soon as possible to avoid possible personal or government embarrassment.

Typically, the surveillance committee will report to the Deputy Minister of Health through its chairperson. The operations personnel likely also will report directly to this senior administrator through the Director of Spray Operations. The Deputy Minister will report, in turn, to the Minister of Health. Monitoring groups, made up of professionals from various organizations and levels of governments, will likely be organized by the Deputy Minister of Environment and will report their findings to that office. The Deputy, of course, will report to the Minister. These and supporting Ministers may form the Core Coordinating Committee or Emergency Task Force and will make recommendations to or be empowered by the Provincial Cabinet. Although these or similar channels may be established, one should expect changes to occur or informal channels to form over the course of the emergency program. Whatever official channels are established, 
the emergency personnel would be well-advised to follow them carefully.

During the emergency, everyone involved, regardless of their level in the temporary organization, will need to be kept up-todate, if not up-to-minute, on all significant findings, occurrences, and activities within their area of responsibility and will be required to summarize and pass on this information and/or recommendations to the person to whom they report. Periodically, especially if the emergency operations are lengthy, they also may be required to provide reports to the various committees. After the emergency is over, they may have to prepare written reports on the activities of their group, highlighting significant data, activities, expenses, etc. Possibly, the reports of the various organizations, agencies, groups, and individuals may be presented at a special symposium or published in a government document. Also, some documents will be required for legal purposes. For these reasons, the need for complete and accurate records and reports and the need for following official reporting procedures is obvious. 


\section{PUBLIC INFORMATION}

a. Implementation

The timely provision of complete and accurate information on the status of the mosquito-borne disease involved, the vector control measures planned, and on personal protection is necessary to maintain public support of the emergency measures being taken. A well-respected public information officer should be appointed to coordinate the distribution of information being provided to the public - through both the news media and telephone inquiry lines. During an encephalitis outbreak, the information coordinator and their staff release information on disease surveillance and control on a daily basis.

As soon as disease surveillance indicates the possibility of a mosquito-borne encephalitis outbreak, the public information program should start. Initially, this may entail the information coordinator being briefed on the activity levels of the virus and mosquito vectors and any concerns that provincial health officials may have that an outbreak may occur.

By involving the information coordinator early, that person is enabled to review contingency plans for dealing with an encephalitis outbreak. Steps can be taken early to update resource files, contact lists, and operational requirements. Colleagues available for help during natural disasters may be alerted in turn. Further, as an outbreak becomes imminent, the information coordinator may begin to attend meetings of the surveillance committee and of health officials to gain a better understanding of the ongoing surveillance and planned control program and to prepare additional draft press releases on all aspects of these interrelated programs.

Alerted to the possibility of an outbreak, the coordinator may be asked to draft press releases on such topics as personal protection from mosquito bites and the status of disease surveillance for issuance by the Minister of Health. Emphasis should be placed on the escalated surveillance program and the importance of the public protecting themselves from mosquito bites. Depending on the government's position on emergency mosquito control, the steps that are under consideration (should the situation deteriorate) may be outlined in the initial statement.

By the time a health emergency is declared, the information coordinator will have organized a facility for an emergency telephone inquiry service, complete with telephones, desks, chairs, map boards, chalk boards, office equipment and supplies, and a coffee-maker. Separate telephone lines should be available for the public, for the media and for internal communication.

Trained professionals should answer the phones. The chosen professionals will likely include public health nurses, medical 
entomologists, agronomists, wildlife biologists, and apiarists who have good public relations skills and can readily assimilate detailed, technical information on all aspects of the disease surveillance and control programs.

Assuming a detailed, information package on the biology and control of the mosquito-borne disease has been prepared by the information coordinator and approved by professional health and vector control officials, this information could be distributed to the media and local government officials early in the public information program, smoothing the way for subsequent information releases.

\section{b. Maintenance Requirements}

Preparation is the key to a good public information program. An information coordinator should only have to update the basic information files once alerted to a possible outbreak of mosquitoborne encephalitis. Neither the government, the media or the public will understand or accept the emergency program if the public information program fails through lack of advance preparation.

\section{Requirements During Non-epidemic Years}

Some of the information maintained between outbreaks is similar to that which would be periodically updated to deal with any emergency situation, natural or otherwise. Other information may be specific to mosquito-borne encephalitis. The basic requirements are outlined below.

\section{Compendium of Questions/Answers}

Ideally, the information coordinator will have developed an information manual for use by the professionals fielding questions from the public through the telephone inquiry line. If an outbreak of mosquito-borne encephalitis has occurred at some time in the past, the questions that the public (and media) will undoubtedly ask will be apparent from a review of old press clippings, publications (e.g. Donogh 1976; Anon. 1976, 1978; Mahdy et al. 1979) and administrative and consultant reports (e.g. Anon. 1983).

Some of the answers may also be obvious. However, many questions may not have been adequately answered in the past. Either they were poorly articulated or understood or current surveillance and control methods and materials make the past answers obsolete. However, anticipating the questions, the information officer can quickly contact his network of experts to obtain accurate, factual, up-to-date answers. Thus, he can prepare good responses to the flood of calls that the inquiry line will receive.

Many of the technical questions will revolve around the human 
safety and environmental impact of the insecticides used. It would be difficult, in a manual such as this, to attempt to answer all the possible questions that might be asked about the dozens of different mosquito control products that might be used alone or in combination during a vector control program. However, it would be useful to list the more-commonly asked questions.

Once it has been decided which insecticide(s) will be used during the health emergency, the answers, specific to those active ingredients, can be developed with the assistance of technical resource people.

Some of the more commonly asked questions follow:

... how safe is this insecticide to me, my children, my pets?

... what is the insecticide being sprayed?

... who manufactures, sells, distributes the insecticide?

... was this insecticide used as a nerve gas during the war?

... wasn't this insecticide banned in the U.S.A.?

... on what other insects is this insecticide registered for use?

... will all the bees, butterflies, lady bugs, dragonflies, be killed?

... what other products could have been used in the larviciding, adulticiding?

... why was this product chosen over the others available?

... is this insecticide safe to the environment?

... will it kill nestlings, insect-eating birds?

... if sprayed over rivers, will it kill the fish?

... is it safe to eat the vegetables, fish, birds, that have been sprayed?

... can I, my kids, my pet be outdoors during the spraying?

... will this insecticide harm the paint/finish on my car?

... does this insecticide cause cancer?

... will this chemical aggravate my asthma, bronchitis, allergies?

The answers to some questions cannot be prepared in advance. These include queries on precisely when and where vector control will be carried out during any given day. The information coordinator must obtain updates on such matters every few hours, recognizing that changing weather conditions may drastically alter vector control plans from hour to hour. Because most of the calls received will centre on the question, "When is spraying planned for my community?", this makes the task of rapidly transferring updated spray schedules a challenge. Also, there will be some questions of a technical nature that either cannot be anticipated or adequately answered by personnel manning the telephones. The information coordinator then must attempt to obtain the precise answers from the experts who do know and relay this information back to the original caller. The specific question/answer can then be added to the compendium. 


\section{List of Technical Resource Persons}

As noted above, some questions cannot be anticipated. Thus, it is vital that the information coordinator maintain a network of professional contacts to whom he can turn when answers are needed quickly.

The listing would include the names, addresses and telephone numbers (day and night) of other information officers. These people might include colleagues in federal, provincial and municipal departments across Canada. During an emergency, these persons, in turn, may be able to obtain needed technical information through professionals in their respective departments.

In addition, the listing would include professionals in provincial and municipal departments of health, environment, and agriculture who have valuable knowledge and experience in fields related to disease surveillance and control. Many of these individuals will likely already be involved, directly or indirectly, in dealing with the outbreak.

\section{Lists of Contacts}

Various contact lists can be developed by the information coordinator for use during the encephalitis outbreak. The contacts who are listed will vary from province to province and from year to year. Computerizing such lists will facilitate scheduled revisions.

\section{Media Contacts}

Because the public information program is dependent on the news media to provide up-to-the-minute information to the public, it is very important that contacts be established with all the news-rooms of major radio and television stations and newspapers in the province.

Such contact lists (complete with names, addresses and telephone numbers) must be updated frequently to remain current and to reflect changes in the organizational structure of the firms and staff.

From public awareness surveysit has been shown that television news is the most effective means of disseminating information to the general public. If any compromises must be made, it should not include this medium

\section{Local Officials}

Lists of municipal and regional contacts may also prove useful during the health emergency. Knowing who the local mayors, reeves, MLA's and MP's are may be helpful, especially when there communities are locations where human/horse cases have been observed and/or which will be aerially sprayed. Early notification of these very important people may be vital to local 
community support.

In addition, it may be helpful to develop special lists of contacts for each community that may be involved in the vector control operations. The lists might include local public health nurses, medical doctors, veterinarians, airport managers, RCMP detachments, etc.

Health Officials

Although it will not normally be necessary for the information coordinator to contact surveillance officials in other jurisdictions, this being done regularly by local surveillance personnel. The following contacts, current in 1989, may prove use ful:

Municipal

Winnipeg

Dr. Doug G. Luckhurst

City of Winnipeg

Health Department

280 William Avenue

Winnipeg, Manitoba R3B OR1

(204) 986-2415

Provincial

Ontario

Dr. Gordon Surgeoner

Department of Environmental Biology

University of Guelph

Guelph, Ontario N1G 2W1

(519) $824-4120$

Manitoba

Dr. Lyla Sekla

Manitoba Health Services Commission

Cadham Provincial Laboratory

750 William Avenue

Winnipeg, Manitoba R3C $3 Y 1$

(204) $944-0270$

Dr. Margaret V. Fast

Manitoba Health

Preventive Medical Services

831 Portage Avenue

Winnipeg, Manitoba R3G ON6

(204) 945-6834 


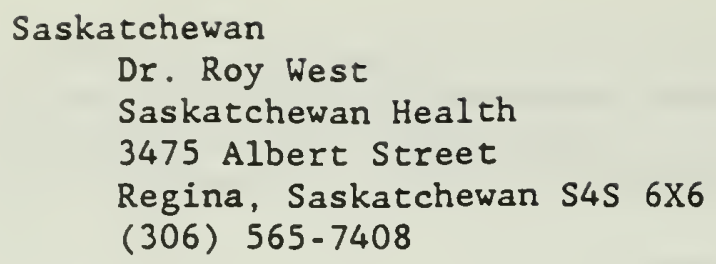

Alberta

Dr. John R. Waters

Alberta Social Services and Community Health Seventh Street Plaza

Edmonton, Alberta T5J 3E4

Federal

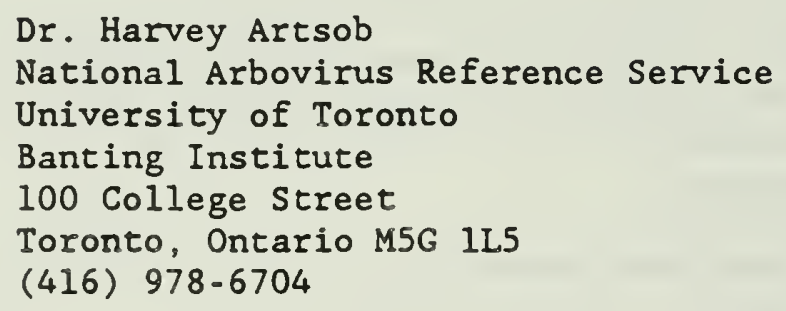

U.S. CDC

Dr. D. Bruce Francy

US Health and Human Services

Public Health Service

Centers for Disease Control

Center for Infectious Diseases

P.0. Box 2087

Fort Collins, Colorado 90522-2087

(303) $221 \cdot 6432$

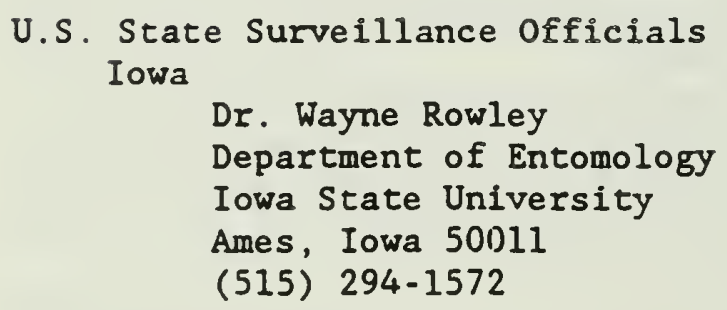


Dr. Michael Osterholm

Minnesota Department of Health

717 Delaware Street Southeast

Minneapolis, Minnesota 55440

(612) $623-5414$

South Dakota

Kenneth A. Senger

Epidemiology

South Dakota Department of Health

Joe Foss Building

Pierre, South Dakota 57501

(605) $773-3364$

North Dakota

Robert Hennes

Division of Environmental Sanitation and Vector Control

North Dakota State Department of Health

State Capital Building

Bismark, North Dakota 58505

Veterinarians

Lists of practising veterinarians in any given province can be obtained from the Agriculture Department, usually the provincial veterinarian or senior pathologist. Such contacts may be helpful in helping TV News film crews obtain footage of such visual elements as, for example, horse vaccinations.

\section{c. Assessment}

A follow-up assessment of the effectiveness of public information is important. In order to improve future such programs, it is worth assessing the various components of the latest program. Few such evaluations have been carried out for public information programs developed for outbreaks of mosquitoborne encephalitis.

One such study was carried out after the 1983 encephalitis outbreak in Manitoba (Anon. 1983). The consultant assessed public perceptions of the health risk presented by WEE, public perceptions of the aerial spraying program, the relative effectiveness of the different media used in the awareness campaign, the public's comprehension of the message elements and any behaviourial impact the campaign produced. It was concluded that the objectives of that public information program were met, in general, but that a significant number of Manitobans did not act on advice to avoid mosquito bites and to avoid exposure to the insecticide spray.

Such weaknesses in the information program, especially 
failing to induce people to protect themselves by wearing mosquito repellents when they are outdoors, underline the need for continual improvement. 
PART III 


\section{REFERENCES CITED}

Adamson, J.D., and S. Dubo. 1942. Clinical findings in encephalitis (Western equine). Can. Publ. Hlth. J. 33: 288300 .

Akesson, N.B., and W.E. Yates. 1982. The use of aircraft for mosquito control. AMCA Bull. 1: 1-96.

Allen, R., S.K. Taylor, and S.E. Sulkin. 1970. Studies of arthropod-borne virus infection in Chiroptera. VIII. Evidence of natural St. Louis encephalitis virus infection in bats. Am. J. Trop. Med. Hyg. 19: 85-91.

Ambrose, A.M., D.K. Huffman, and R.T. Salamone. 1959.

Pharmacologic and toxicologic studies on $\mathrm{N}, \mathrm{N}$

diethyltoluamide. I. Toxicol. Appl. Pharmacol. 1: 97-115.

Anderson, A.W., and R.F. Harwood. 1966. Cold tolerance in adult female Culex tarsalis (Coquillett). Mosquito News 26: 1-7.

Andrews, W.N., W.A. Rowley, Y.W. Wong, D.C. Dorsey, and W.J. Hausler. 1977. Isolation of trivittatus virus from larvae and adults reared from field-collected larvae of Aedes trivittatus (Diptera: Culicidae). J. Med. Entomol. 13: 699701 .

Anonymous. 1965. Prevention and control of vector problems associated with water resources. USHEW, PHS, CDC. 26 PP.

Anonymous. 1974. Equipment for vector control. WHO Manual, Geneva, Switzerland. 179 pp.

Anonymous. 1976. Control of St. Louis Encephalitis. USHEW/CDC Vector Topics No. 1. 35 pp.

Anonymous. 1977. Engineering aspects of vector control operations. lst Rpt. WHO Expert Comm. on Vector Biology and Control, Tech. Rpt. Series 603: 1-40.

Anonymous. 1978. Control of Western Equine Encephalitis. USHEW/CDC Vector Topics No. 3. 35 pp.

Anonymous. 1982. Emergency vector control after natural disaster. Pan American Hlth. Assoc. 98 pp. 
Anonymous. 1983. Western equine encephalitis public information program evaluation survey: final report. Criterion Research Corp. 25 pp. + appendix.

Anonymous. 1987. California's mosquitoborne encephalitis virus surveillance and control program. California Mosquito and Vector Control Association and UC Berkeley. 14 pp. + appendices.

Anonymous. 1989. Mosquitoes in Illinois: recommendations for prevention and control. Illinois Dept. Public Hlth. 30 pp.

Armstrong, J.A. 1977. Relationship between the rates of pesticide application and the quantity deposited on the forest. In: J.R. Roberts, R. Greenhalgh, and W.K. Marshall (Eds.) Proc. Symp. Fenitrothion: The Long Term Effects of its Use in Forest Ecosystems. National Research Council, Ottawa. pp. $159-182$.

Arntfield, P.W., and W.J. Gallaway. 1982. Blood-feeding in overwintering Culex tarsalis (Diptera: Culicidae) from Manitoba. Can. Ent. 114: 85-86.

Artsob, H. 1983. Distribution of California serogroup viruses and virus infections in Canada. In: Calisher, C.H., and W. H. Thompson (Eds.). California Group Viruses. pp. 277-290.

Artsob, H., and L. Spence. 1979. Arboviruses in Canada. In: Kurstak, E. (Ed.). Arctic and Tropical Arboviruses. Academic Press. Chapter 4. pp. 39-65.

Artsob, H., L.P. Spence, C.H. Calisher, L.H. Sekla, and R.A. Brust. 1985. Isolation of California encephalitis serotype from mosquitoes collected in Manitoba, Canada. J. Am. Mosq. Control Assoc. 1: 257-258.

Artsob, H., L. Spence, and C. Th'ng. 1979a. Serological survey of Niagara and Windsor residents for arbovirus antibodies. In: M.S. Mahdy, L. Spence, and J.M. Joshua (Eds.). Arboviral Encephalitides in Ontario With Special Reference to St. Louis Encephalitis. Ontario Ministry of Health. pp. 149-162.

Artsob, H., L. Spence, and C. Th'ng. 1979b. Horses as monitors for arboviral activity in southern Ontario. In: M.S. Mahdy, L. Spence, and J.M. Joshua (Eds.). Arboviral Encephalitides in Ontario with Special Reference to St. Louis Encephalitis. Ontario Ministry of Health. pp. 233-243. 
Atkins, E.G. 1975. Injury to honey bees by poisoning. In: Dadant \& Sons (Eds.). The Hive and the Honey Bee. Dadant \& Sons, Hamilton, Illinois.

Bailey, C.L., B.F. Eldridge, D.E. Hayes, D.M. Watts, R.F. Tammarie1lo, and J.M. Dalrymple. 1978. Isolation of St. Louis encephalitis virus from overwintering Culex pipiens mosquitoes. Science 199: 1348-1349.

Bailey, S.F., D.A. Ellason, and B.L. Hoffmann. 1965. Flight and dispersal of the mosquito Culex tarsalis Coquillett in the Sacramento Valley of California. Hilgardia 37: 73-113.

Banks, A., and R.W. Russe11. 1967. Effects of chronic reductions in acetylcholinesterase activity on serial problem-solving behavior. J. Comp. Physiol. Psychol. 64: 262-267.

Barrett, M.W., and G.A. Chalmers. 1975. A serologic survey of pronghorns in Alberta and Saskatchewan, 1970-1972. J. Wildlife Dis. 11: 157-163.

Beadle, L.D. 1952. Eastern equine encephalitis in the United States. Mosquito News 12: 102-107.

Bellamy, R.E., and W.C. Reeves. 1963. The winter biology of Culex tarsalis (Diptera: Culicidae) in Kern County, California. Ann. Entomol. Soc. Am. 56: 314-323.

Bellamy, R.E., W.C. Reeves, and R.P. Scrivani. 1958. Relationships of mosquito vectors to winter survival of encephalitis viruses. II. Under experimental conditions. Am. J. Hyg. $67: 90-100$.

Bellamy, R.E., W.C. Reeves, and R.P. Scrivani. 1967. Experimental cyclic transmission of western equine encephalitis virus in chickens and Culex tarsalis through a year. Am. J. Epidemio1. 85: 282-296.

Bellance, R., E. Rossier, M. Lemaitre, N.G. Willis, and P. Bellander. 1973. Eastern equine encephalomyelitis in Eastern Canada. Am. J. Publ. H1th. 64: 189-190.

Bennington, E.E., J.S. Blackmore, and C.A. Sooter. 1958a. Soil temperature and the emergence of Culex tarsalis from hibernation. Mosquito News 18: 297-298.

Bennington, E.E., C.A. Sooter, and H. Baer. 1958b. The diapause in adult female Culex tarsalis Coquillett. Mosquito News.18: 
Berry, R.L., B.J. LaLande Weigert, C.H. Calisher, M.A. Parsons, and G.T. Bear. 1977. Evidence of transovarial transmission of Jamestown Canyon virus in Ohio. Mosquito News 37: 494-496.

Bidlingmayer, W.L. 1985. The measurement of adult mosquito population changes - some considerations. J. Am. Mosq. Control Assoc. 1: 328-348.

Blackmore, J.S., and R.P. Dow. 1962. Nulliparity in summer and fall populations of Culex tarsalis Coquillett. Mosquito News 22: $291-294$.

Blackmore, J.S., and J.F. Winn. 1956. A winter isolation of western encephalitis virus from hibernating Culex tarsalis Coquillett. Proc. Soc. Exp. Biol. Med. 91: 146-148.

Blomquist, L., and W. Thorse11. 1977. Distribution and fate of insect repellent ${ }^{14} \mathrm{C}-\mathrm{N}, \mathrm{N}$ diethyl-m-toluamide in the animal body: II. Distribution and excretion after cutaneous application. Acta Pharmacol. Toxicol. 41: 235-243.

Bowen, G.S., and D.B. Francy. 1980. Surveillance. In: Monath, T.P. (Ed.). 1980. St. Louis Encephalitis. American Public Health Association, Washington, D.C. Pp. 473-499.

Brennan, J.M., and R.F. Harwood. 1953. A preliminary report of the laboratory colonization of the mosquito, Culex tarsalis Coquillett. Mosquito News 13: 153-157.

Bristow, G.E. 1979. Climatological conditions in southwestern Ontario in 1975. Their possible impact on the outbreak of St. Louis Encephalitis. In: Mahdy, M.S., L.S. Spence and J.M. Joshua (Eds.). Arboviral Encephalitides in Ontario With Special Reference to St. Louis Encephalitis. Ontario Ministry of Health. pp. 70-77.

Brown, A.W.A. 1978. Ecology of Pesticides. John Wiley and Sons. 525 pp.

Brust, R.A. 1982. Population dynamics of Culex tarsalis Coquillett in Manitoba. In: Sekla, L. (Ed.). 1982. Western Equine Encephalitis in Manitoba. Manitoba Health Services Commission. pp. 21-30.

Brust, R.A. 1984. Mosquito Control Evaluations. In: Final Technical Report Volume Environmental Monitoring Program for 
the 1983 Aerial Spraying of Malathion to Combat Western Equine Encephalitis. Manitoba Environment, Workplace Safety and Health. pp. 189-225.

Brust, R.A., and R.A. Ellis. 1976a. Mosquito surveys in Manitoba during 1975. Can. J. Publ. H1th. 67 (Suppl.): 47-53.

Brust, R.A., and R.A. Ellis. 1976b. Assessment of the emergency mosquito control operation in Manitoba, 1975. Can. J. Publ. H1th. 67 (Supp1.): 69-71.

Brust, R.A., R.A. Ellis, and K.W. Plews. 1976. Guidelines for mosquito control in Manitoba. Manitoba Mines, Resources, and Environmental Management. 19 pp.

Burdick, D.J., and E.H. Kardos. 1963. The age structure of fall, winter, and spring populations of Culex tarsalis in Kern County, California. Ann. Entomol. Soc. Am. 56: 527-535.

Burgoyne, W.E., and N.B. Akesson. 1971. The aircraft as a tool in large scale vector control programs. Int. Agr. Avia. Centre, Cranfield, England.

Burton, A.N., R. Connell, J.G. Rempel, and J.B. Gallop. 1961. Studies on western equine encephalitis associated with wild ducks in Saskatchewan. Can. J. Microbio1. 7: 205-302.

Burton, A.N., and J. Mclintock. 1970. Further evidence of western encephalitis infection in Saskatchewan mammals and birds and in reindeer in northern Canada. Can. Vet. J. 11: 232-235.

Burton, A.N., J. Mclintock, and D.B. Francy. 1973. Isolation of St. Louis encephalitis and Cache Valley viruses from Saskatchewan moquitoes. Can. J. Pub1. H1th. 64: 368-73.

Burton, A.N., J. Mclintock, and J.G. Rempe1. 1966a. Western equine encephalitis virus in Saskatchewan garter snakes and leopard frogs. Science 154: 1029-1031.

Burton, A.N., J. Mclintock, J. Spalatin, and J.G. Rempel. 1966b. Western equine encephalitis in Saskatchewan birds and mammals 1962-1963. Can. J. Zoo1. 12: 133-141.

Buth, J.L. 1983. The bionomics of three potential vectors of Western Equine Encephalitis in Manitoba. M.Sc. Thesis, University of Manitoba. 112 pp.

Calisher, C.H. 1983. Taxonomy, classification and geographic 
distribution of California group bunyaviruses. In:

Calisher, C.H., and W.H. Thompson (Eds.). California Group Viruses. pp. 1-16.

Calisher, C.H., T.P. Monath, D.J. Moth, J.S. Lazuick, D.W. Trent, D.B. Francy, G.E. Kemp, and F.W. Chandler. 1980.

Characterization of Fort Morgan virus, an alphavirus of the western equine encephalitis complex in an unusual ecosystem. Am. J. Trop. Med. Hyg. 29: 1428-1440.

Cameron, G.D.W. 1942. Western equine encephalitis. Can. Publ. H1th. J. 33: 383-387.

Canadian Water Quality Guidelines. 1987. Canadian Council of Resource and Environment Ministers. Environment Canada, Ottawa.

Centers for Disease Control. 1985. Arboviral infections of the central nervous system, United States 1984. Morbidity \& Mortality Weekly Rpt. 34: 283.

Chamberlain, R.W. 1980. History of St. Louis Encephalitis. In: St. Louis Encephalitis. Monath, T.P. (Ed.). American Public Health Association, Washington, D.C. Chapt. 1. pp. 3-61.

Chamberlain, R.W. 1987. Historical perspectives on the epidemiology and ecology of mosquito-borne virus encephalitides in the United States. Am. J. Trop. Med. Hyg. 37 (Supp1.) : 8-17.

Chamberlain, R.W., R.E. Kessling, D.D. Stamin, and W.D. Sudia. 1957. Virus of St. Louis Encephalitis virus in three species of wild birds. Am. J. Hyg. 65: 110-118.

Chamberlain, R.W., and W.D. Sudia. 1961. Mechanism of transmission - of viruses by mosquitoes. Ann. Rev. Entomol. 6: 371-390.

Chamberlain, R.W., D.W. Sudia, and J.D. Gillett. 1959. St. Louis encephalitis virus in mosquitoes. Am. J. Hyg. 70: 221-236.

Chant, G.D., W.F. Baldwin, and L. Forster. 1973. Occurrence of Culiseta melanura (Diptera:Culicidae) in Canada. Can. Ent. 105: 1359 .

Chapman, H.C. 1961. Abandoned mines as overwintering sites for mosquitoes, especially Culex tarsalis Coquillett in Nevada. Mosquito News 21: $324-327$. 
Clark, G.G., T.H. Work, and O.G.W. Berlin. 1976. Environmental influences on CDC light trap collections of Culex tarsalis in a focus of WEE and SLE transmission. Proceedings and Papers of the Annual Conference of California Mosquito Control Association 44: 33-40.

Clarke, J.L. 1943. Studies of the flight range of mosquitoes. J. Econ. Entomol. 36: 121-122.

Copps, P.T., G.A. Surgeoner, and B.V. Helson. 1984. An assessment of sampling techniques for adult mosquitoes in southern Ontario. Proc. entomol. Soc. Ont. 115: 61-70.

Crane, G.T., R.E. Elbel, and C.H. Calisher. 1977. Transovarial transmission of California encephalitis virus in the mosquito Aedes dorsalis at Blue Lake, Utah. Mosquito News 37: 479482 .

Crans, W.J. 1977. The status of Aedes sollicitans as an epidemic vector of eastern equine encephalitis in New Jersey. Mosquito News 37: 85-89.

Cyanamid of Canada, Ltd. 1970. Abate 4-E for control of black fly and mosquito larvae.

Davidson, W.G., Snell, E., Joshua, J.M., and R. West. 1976. Human arboviral infection in Canada in 1975. Can. Dis. Weekly Rept. 2-21: $81-83$.

Davison, R.0. 1942. Encephalitis in Saskatchewan, 1941 (Preliminary report). Can. Publ. Hlth. J. 33: 388-398.

Deibel, R., S. Srihongse, M.A. Grayson, P. Grimstad, M.S. Mahdy, H. Artsob, and C.H. Calisher. 1983. Jamestown Canyon virus: the etiological agent of an emerging human disease? In: Calisher, C.H., and W.H. Thompson (Eds.). California Group Viruses.

Dillenberg, H. O. 1965. Western equine encephalomyelitis (WEE) in Saskatchewan. Can. J. Pub. H1th. 56: 17-20.

Dillenberg, H.O., M.S. Acker, R.J.P. Belcourt, and F.P. Nagler. 1956. Some problems in the epidemiology of neurotropic virus infections. Can. J. Publ. H1th. 47: 6-14.

Dixon, D., and B. Fingler. 1982. The affects of the 1981 Manitoba emergency mosquito control program on honey bees. In: Sekla, L. (Ed.). Western Encephalitis in Manitoba. Manitoba 
Health Services Commission. pp. 243-247.

Dixon, D.P., and B. Fingler. 1984. The effects of the mosquito control program on bees. In: Final Technical Report Volume. Environmental Monitoring Program for the 1983 Aerial Spraying of Malathion to Combat Western Equine Encephalitis. Manitoba Environment, Workplace Safety and Health. pp. 101-121.

Dominion Bureau of Statistics. 1941. Census of Canada. pp. 6-7.

Donogh, N.R. 1976. Public information on Western Encephalomyelitis and emergency mosquito control in Manitoba, 1975. Can. J. Pub1. H1th. 67 (Supp1. 1): 61-62.

Donovan, C.R., and M. Bowman. 1942a. Some epidemiological features of poliomyelitis and encephalitis, Manitoba, 1941. Can. Publ. Hlth. J. 33: 246-257.

Donovan, C.R., and M. Bowman. 1942b. Epidemiology of encephalitis: western equine type, Manitoba, 1941. Can. Med. Assoc. J. 46: $525-537$.

Dorland, R., M.S. Mahdy, H. Artsob, and A. Prytula. 1979. Wild bird surveillance in the study of arboviral encephalitis. In: Mahdy, M.S., L. Spence, and J.M. Joshua (Eds.). 1979. Arboviral Encephalitides in Ontario With Special Reference to St. Louis Encephalitis. Ontario Ministry of Health. pp. 208224.

Dow, R.P., L.C. LaMotte, Jr., and G.T. Crane. 1976. Posthibernating Culex tarsalis and Culiseta inornata: oviparity and tests for virus. Mosquito News 36: 63-68.

Draisey, T.F., M. Dretrech, and M.J. Oxley. 1979. Pathology of the central nervous system in arboviral encephalitides. Case reports of St. Louis Encephalites. In: Arborival Encephalitides in Ontario with Special Reference to St. Louis Encephalitis. Ontario Ministry of Health. pp. 122-136.

Eadie, J., and B. Friesen. 1982. Epidemiological study of western equine encephalitis in 1981. In: Sekla, L. (Ed.). Western equine encephalitis in Manitoba. Manitoba Health Services Commission. pp. 142-155.

Earnst, M.P., H.A. Goolishian, J.R. Calverley, R.G. Hayes, and H.R. Hill. 1971. Neurologic, intellectual, and psychologic sequelae following western encephalitis. Neurology 21: 969974. 
Edman, J.D., and D.J. Taylor. 1968. Culex nigripalpus: seasonal shift in the bird-mammal feeding ratio in a mosquito vector of human encephalitis. Science 161: 67-68.

Eldridge, B.F. 1968. The effect of temperature and photoperiod on blood-feeding and ovarian development in mosquitoes of the Culex pipiens complex. Am. J. Trop. Med. Hyg. 17: 133-140.

Eldridge, B.F.1987a. Diapause and related phenomena in Culex mosquitoes: their relation to arbovirus disease ecology. In: Harris, K.F. (Ed.) Current topics in vector research. Vol. 4: 1-28. Springer Verlag, New York.

Eldridge, B.F. 1987b. Strategies for vector surveillance, prevention, and control of arboviruses in western North America. Am. J. Trop. Med. Hyg. 37 (suppl.): 77-86.

Eldridge, B.F., and C.L. Bailey. 1979. Experimental hibernation studies in Culex pipiens (Diptera: Culicidae): reactivation of ovarian development and blood-feeding in prehibernating females. J. Med. Entomol. 15: 462-467.

Ellis, R.A. 1976. Emergency measures and mosquito control operations during the 1975 Western encephalomyelitis outbreak in Manitoba. Can. J. Publ. Hlth. 67(Suppl.): 59-60.

Ellis, R.A. 1982a. Emergency mosquito vector control in Manitoba. Part 1. Aerial ULV application of insecticide for large area vector control. Prairie Pest Management. 188 pp.

Ellis, R.A. 1982b. Emergency mosquito vector control in Manitoba. Part 2. Mosquito control by rural municipalities.

Ellis, R.A. 1982c. Emergency mosquito vector control in Manitoba. Part 3. Public relations information manual. Prairie Pest Management. $83 \mathrm{pp}$.

E11is, R.A. 1984. Annual report on mosquito surveillance and control in Winnipeg-1984. Insect Control Branch, Parks and Recreation, City of Winnipeg.

Ellis, R.A., and R.A. Brust. 1982. Effectiveness of the emergency mosquito vector control operations. In: Sekla, L. (Ed.). 1982. Western Equine Encephalitis in Manitoba. Manitoba Health Sciences Commission. pp. 209-222.

Ellis, R.A., and D.M. Wood. 1974. First Canadian record of 
Corethrella brakeleyi (Diptera: Chaoboridae). Can. Ent. 106: $221-222$.

Fabian, R., Griffin, T.B., and F. Coulston. 1971. Occurrence of insecticidal chlorinated hydrocarbons and their breakdown products in man and the resulting toxicologic consequences. In: Tahori, A.S. (Ed.). Pesticide Terminal Residues. International Union of Pure and Applied Chemistry. Butterworths, London. pp. 145-160.

Feldlaufer, M.F., and W.J. Crans. 1979. The relative attractiveness of carbon dioxide to parous and nulliparous mosquitoes. J. Med. Entomol. 15: 140-142.

Feltz, E.T., B. List-Young, D.G. Ritter, P. Holden, G.R. Noble, and P.S. Clark. 1972. California encephalitis virus: serological evidence of human infections in Alaska. Can. J. Microbio1. 18: 757-762.

Ferguson, F.F. 1954. Biological factors in the transmission of arthropod-borne virus encephalitides. Publ. Hlth. Monogr. 23: $1-37$.

Fox, J. 1982. The case for control of Western equine encephalitis in humans through vaccination. In: Sekla, L. (Ed.). Western Equine Encephalitis in Manitoba. Manitoba Public Services Commission. pp. 259-263.

Francy, D.B., W.A. Rush, M. Montoya, D.S. Inglish, and R.A. Bolin. 1981. Transovarial transmission of St. Louis encephalitis virus by Culex pipiens complex mosquitoes. Am. J. Trop. Med. Hyg. 30: 699-705.

Fraser, H. 1982. Summer weather in Manitoba, 1981. In: Sekla, I. (Ed.). 1982. Western Equine Encephalitis in Manitoba. Manitoba Health Services Commission. pp. 31-35.

Fraser, J.M., and R.A. Brust. 1976. Weather conditions affecting mosquito populations in southern Manitoba during 1975. Can. J. Pub1. H1th. 67 (Supp1.) : 40-46.

Friesen, B., and J. Eadie. 1982. Clinical study of western equine encephalitis in 1981. In: Sekla, L. (Ed.). Western Equine Encephalitis in Manitoba. Manitoba Health Services Commission. pp. 118-128.

Fulton, J.S. 1938. A report of two outbreaks of encephalomyelitis in Saskatchewan. Can. J. Comp. Med. 11: 39-46. 
Fulton, J.S. 1941. Relation of equine encephalomyelitis to the epidemic of human encephalitis in Saskatchewan in 1938. Can. Publ. Hlth. J. 32: 6-12.

Fulton, J.S., and A.N. Burton. 1953. After effects of western equine encephalitis in man. Can. Med. Assoc. J. 69: 268272 .

Fusse11, E.M. 1964. Dispersal studies on radioactive-tagged Culex quinquefasciatus Say. Mosquito News 24: 422-426.

Gallaway, W.J. 1983. Ecology and overwintering biology of potential mosquito vectors of Western Equine Encephalitis in Manitoba. M.Sc. Thesis, University of Manitoba. 80 pp.

Gahlinger, P., W.C. Reeves, and M.M. Milby, 1986. Air conditioning and television as protective factors in arboviral encephalitis risk. Am. J. Trop. Med. 35: 601-610.

Gardner, D.R., and J.R. Bailey. 1975. Methoxychlor: its effects on environmental quality. National Research Council of Canada, NRCC 14102. 164 pp.

Gareau, U. 1941. Clinical aspects of an epidemic of human encephalomyelitis in Saskatchewan in 1938. Can. Publ. Hlth. J. $32: 1-5$.

Gartrell, M.J., J.C. Craun, D.S. Podrebarac, and E.L. Gunderson. 1986. Pesticides, selected elements, and other chemicals in adult total diet samples, October 1980-March 1982. J. Assoc. Offic. Agric. Chem. 69: 146-161.

Geery, P.R., R.E. Holub, and R.R. Keen. 1983. Statistical evaluation of ground applied ULV Malathion on natural populations of Aedes vexans and Culex species. Mosquito News 43: $206-208$.

Georghiou, G.P. 1986. The magnitude of the resistance problem. In: Pesticide Resistance: Strategies and Tactics for Management. National Academy Press, Washington. pp. 14-43.

Gilbert, I.H. 1957. Evaluation of repellents against mosquitoes and deerflies in Oregon. J. Econ. Entomol. 50:46-48.

Gilbert, I.H., J.L. Gouck, and C.N. Smith. 1957. New mosquito repellents. J. Econ. Entomol. 48: 741-743. 
Gjullin, C.M., T.D. Mulhern, and R.C. Husbands. 1963. The daily resting cycle of several species of mosquitoes. Mosquito News 23: $203-210$.

Glotfelty, D.E., J.N. Seiber, and L.A. Liljedahl. 1987, Pesticides in fog. Nature 325: 602-605.

Goulk, H.K., D.R. Godwin, K. Posey, C.E. Schreck, and D.E. Weidhaas. 1971. Resistance to aging and rain of repellenttreated netting used against salt marsh mosquitoes in the field. Mosquito News 31: 95-99.

Greech, W.B. 1977. St. Louis encephalitis in the United States, 1975. J. Infect. Dis. 135: 1014.

Grimstad, P.R. 1983. Mosquitoes and the incidence of encephalitis. Adv. Virus Res. 28: 357-439.

Gubler, D.J., R. Novak, and C.J. Mitche11. 1982. Arthropod vector competence - epidemiological, genetic, and biological considerations. In: Steiner, W.W., W.J. Tabachnick, K.S. Rai, and S. Nararg (Eds.). 1982. Recent Developments in the Genetics of Insect Disease Vectors. Stipes Publishing Company, Champaign, I11. Pp. 343-378.

Gwatkin, R., and I.W. Moynihan. 1942. Search for sources and carriers of equine encephalitis virus. Can. J. Res. C. 20: $321-337$.

Hagstrum, D.W., and E.B. Workman. 1971. Interaction of temperatures and feeding rate in determining the rate of development of larval Culex tarsalis (Diptera: Culicidae). Ann. Entomo1. Soc. Am. 64: 668-671.

Hammon, W.MCD., and W.C. Reeves. 1952. California encephalitis virus, a newly described agent. Calif. Med. 77: 303-309.

Hammon, W.McD., W.C. Reeves, S.R. Benner, and B. Brookman. 1945. Human encephalitis in the Yakima Valley, Washington, 1942, with forty-nine virus isolations (Western Equine and St. Louis types) from mosquitoes. J. Am. Med. Assoc. 128: 11331139 .

Hardy, J.L., G. Apperson, S.M. Asman, and W.C. Reeves. 1978. Selection of a strain of Culex tarsalis highly resistant to infection following ingestion of Western Equine Encephalomyelitis virus. Am. J. Trop. Med. Hyg. 27: 313- 
Hardy, J.L., and J.P. Bruen. 1974. Aedes melanimon as a vector of WEE virus in California. Proceedings and Papers of the 42nd Annual Conference of the California Mosquito Control Association 42: 36 .

Hardy, J.L., E.J. Houk, L.D. Kramer, and W.C. Reeves. 1983. Intrinsic factors affecting vector competence of mosquitoes for arboviruses. Ann. Rev. Entomol. 28: 229-62.

Hardy, J.L., W.C. Reeves, J.P. Bruen, and S.R. Presser. 1979. Vector competence of Culex tarsalis and other mosquito species for Western Equine Encephalomyelitis virus. In: Kurstak, E. (Ed.). 1979. Arctic and Tropical Arboviruses. Academic Press, New York. pp. 157-171.

Hardy, J.L., L. Rosen, L.D. Kramer, S.B. Presser, D.A. Shrazer, and M.J. Turel1. 1981. Effect of rearing temperature on transovarial transmission of St. Louis encephalitis virus in mosquitoes. Am. J. Trop. Med. Hyg. 29: 963-968.

Hardy, J.L., L. Rosen, W.C. Reeves, R.P. Scrivani, and S.B. Presser. 1984. Experimental transovarial transmission of $\mathrm{St}$. Louis encephalitis virus by Culex and Aedes mosquitoes. Am. J. Trop. Med. Hyg. 33: 166-175.

Harwood, R.F. 1962. Trapping overwintering adults of the mosquitoes Culex tarsalis and Anopheles freeborni. Mosquito News 22: 26-31.

Harwood, R.F., and E. Halfhil1. 1964. The effect of photoperiod on fat body and ovarian development of Culex tarsalis (Diptera: Culicidae). Ann. Entomol. Soc. Am. 57: 596-600.

Harwood, R.F., and N. Takata. 1965. Effect of photoperiod and temperature on fatty acid composition of the mosquito Culex tarsalis. J. Insect Physiol. 11: 711-716.

Hatfield, C.T., and J.M. Anderson. 1972. Effects of two insecticides on the vulnerability of Atlantic salmon (Salmo salar) parr to brook trout (Salvelinus fontinalis) predation. J. Fish. Res. Bd. Can. 29: 27-29.

Hawryluk, G., G. Hammond, and B. Friesen. 1981. Neuropsychological sequelae after western equine encephalitis infection. In: Sekla, L. (Ed.). Western equine encephalitis in Manitoba. Manitoba Health Services Commission. pp. 129-141. 
Hayes, C.G., and R.C. Wallis. 1977. Ecology of western equine encephalomyelitis in the eastern United States. Adv. Virus Res. 21: 37-83.

Hayes, R.O. 1961. Host preferences of Culiseta melanura and allied mosquitoes. Mosquito News 21: 179-187.

Hayes, R.O., J.B. Daniels, K.S. Anderson, M.A. Parsons, H.K. Maxfield, and L.C. LaMotte. 1961. Detection of eastern encephalitis virus and antibody in wild and domestic birds in Massachusetts. Am. J. Hyg. 75: 183-189.

Hayes, R.O., L.C. LaMotte, and P. Holden. 1967. Ecology of arboviruses in Hale County, Texas, during 1955. Am. J. Trop. Med. Hyg. 16: 675-687.

Hayes, W.J., Jr. 1975. Toxicology of Pesticides. Williams and Wilkins, Baltimore. 580 pp.

Hayles, L.B. 1976. Amount of Western Equine Encephalitis virus inoculated by transmitting Culex tarsalis mosquitoes. Res. vet. Sci. 21: 358-359.

Hayles, L.B., J. McLintock, and J.R. Saunders. 1972. Laboratory studies on the transmission of Western Equine Encephalitis virus by Saskatchewan mosquitoes. I. Culex tarsalis. Can. J. Comp. Med. 36: 83-88.

Helson, B.V., G.A. Surgeoner, J. Thorsen, and R.E. Wright. 1979a. Antibody surveillance of sentinel chickens for monitoring arbovirus activity in Ontario. In: Mahdy, M.S., L. Spence, and J.M. Joshua, (Eds.). Arboviral Encephalitides in Ontario With Special Reference to St. Louis Encephalitis. Ontario Ministry of Health. pp. 225-243.

Helson, B.V., G.A. Surgeoner, and R.E. Wright. 1979b. Mosquitoes of southwestern Ontario, their seasonal distribution, prevalence and new records. In: Mahdy, M.S., L. Spence, and J.M. Joshua, (Eds.). Arboviral Encephalitides in Ontario With Special Reference to St. Louis Encephalitis. Ontario Ministry of Health. pp. 181-198.

Henderson, L.P., R.A. Brust, and F.C. Wong. 1979. Biological transmission of Western Encephalomyelitis virus by Culex tarsalis Coquillett. Mosquito News 39: 385-390.

Herbold, J.R., Heuschele, W.P. Berry, R.L., and M.A. Parsons. 
1983. Reservoir of St. Louis encephalitis virus in Ohio bats. Am. J. Vet. Res. 44: 1889-1893.

Hess, A.D., C.E. Cherubin, and L.C. LaMotte. 1963. Relation of temperature to activity of Western and St. Louis Encephalitis viruses. Am. J. Trop. Med. Hyg. 12: 657-667.

Hess, A.D., and R. O. Hayes. 1967. Seasonal dynamics of Western Encephalitis virus. Am. J. Med. Sci. 253: 333-348.

Hess, A.D., and P. Holden. 1958. The natural history of the arthropod-borne encephalitides in the United States. Ann. N.Y. Acad. Sci. 70: 294-311.

Hoff, G.L., T.M. Yuill, J.O. Iversen, and R.P. Hanson. 1969. Snowshoe hares and the California encephalitis group in Alberta, 1961-1968. Bull. Wildl. Dis. Assoc. 5: 254.

Hoff, G.L., T.M. Yuil1, J.O. Iversen, and R.P. Hanson. 1970. Selected microbial agents in snowshoe hares and other vertebrates of Alberta. J. Wildl. Dis. 6: 472-478.

Holden, P., R.O. Hayes, C.J. Mitche11, D.B. Francy, J.S. Lazuick, and T.B. Hughes. 1973. House sparrows, Passer domesticus (L.) as hosts of arboviruses in Hale County, Texas. I. Field studies. 1965-1969. Am. J. Trop. Med. Hyg. 22: 244-253.

Howard, J.J., D.E. Emord, and C.D. Morris. 1983. Epizootiology of eastern equine encephalomyelitis virus in upstate New York, USA. V. Habitat preference of host-seeking mosquitoes (Diptera: Culicidae). J. Med. Entomol. 20:62-69.

Houk, E.J., L.D. Kramer, J.L. Hardy, and S.L. Presser. 1986. An interspecific mosquito model of the mesenteron infection barrier to Western Equine Encephalitis virus (Culex tarsalis and Culex pipiens). Am. J. Trop. Med. Hyg. 35: 632-641.

Howitt, B.F., H.R. Dodge, L.K. Bishop, and R.H. Garrie. 1949. Recovery of the virus of eastern equine encephalomyelitis from mosquitoes (Mansonia perturbans) collected in Georgia. Science 110: 141-142.

Howitt, B.F. 1938. Recovery of the virus of equine encephalomyelitis from the brain of a child. Science 88: 455 .

Hudson, J.E. 1978. Overwintering sites and ovarian development of some mosquitoes in central Alberta, Canada. Mosquito News 
Hughes, D.N., M.G. Boyer, M.H. Papst, C.D. Fowle, G.A.V. Rees, and P. Baulu. 1980. Persistence of three organophosphorus insecticides in artificial ponds and some biological implications. Arch. Environ. Contam. Toxicol. 9: 269-279.

Hurlbert, S. 1975. Secondary effects of pesticides on aquatic ecosystems. Residue Reviews 58: 81-148.

Iversen, J.O., R.J. Wagner, C. DeJong, and J. McLintock. 1973. California encephalitis in Saskatchewan. Isolation from boreal Aedes mosquitoes. Can. J. Pub1. H1th. 64: 590-594.

Jackson, F.W. 1942. Encephalitis. Can. Med. Assoc. J. 46: $364-365$.

Jacobson, J.L., and S.W. Jacobson. 1988. New methodologies for assessing the effects of prenatal toxic exposure on cognitive functioning in humans. In: Evans, M.S. (Ed.). Toxic Contaminants and Ecosystem Health: A Great Lakes Focus. J. Wiley and Sons. pp. 373-388.

Jegier, Z. 1969. Pesticide residues in the atmosphere. Ann. N.Y. Acad. Sci. 160: 143-154.

Johansen, C.A. 1977. Pesticides and pollinators. Ann. Rev. Entomo1. 22: 177-192.

Johnson, W.W., and M.T. Finley. 1980. Handbook of acute toxicity of chemicals to fish and aquatic invertebrates. U.S. Fish and Wildlife Service. Resource Pub. 137. 98 pp.

Jones, J.R. 1979. St. Louis Encephalitis (SLE) in Windsor-Essex Co. Ont., 1975. In: Mahdy, M.S., L. Spence, and J.M. Joshua (Eds.). Arboviral Encephalitides in Ontario With Special Reference to St. Louis Encephalitis. Ontario Ministry of Health. pp. 78-94.

Joshua, J.M. 1979. Clinical and epidemiological aspects of encephalitis in Ontario 1975-76. A composite picture. In: Mahdy, M.S., L. Spence, and L.M. Joshua (Eds.). Arboviral Encephalitides in Ontario with Special Reference to St. Louis Encephalitis. Ontario Ministry of Health. pp.137-148.

Khan, A. A., H.I. Maibach, and D.L. Skidmore. 1973. Insect repellents. 2. Effect of temperature on protection time. J. Econ. Entomol. 6: 437-438. 
Karabatsos, N. 1980. General characterstics and antigenic relationships. In: St. Louis Encephalitis, Monath, T.P. (Ed.). Am. Public Health Assoc., Washington, D.C. Chapt. 3. pp. $105-158$.

Keener, G.G., Jr. 1952. Observations on overwintering of Culex tarsalis Coquillett (Diptera: Culicidae) in western Nebraska. Mosquito News 12: 205-209.

Kemp, G.E. 1981. St. Louis Encephalitis (SLE). In: CRC Handbook Series in Zoonoses, Sect. B, Viral Zoonoses. pp. 71-83.

Kettyls, G.D., and E.J. Bowmer. 1975. Western equine encephalitis. Epidemiol. Bull. 19: 24.

Kettyls, G.D., V.M. Verral1, L.D. Wilton, J.B. Clapp, D.A. Clarke, and J.D. Rubblee. 1972. Arbovirus infections in man in British Columbia. Can. Med. Assoc. J. 106: 1175-1179.

Kliewer, J.W., T. Miura, and H.C. Chapman. 1969. Seasonal occurrence and physiology of Culex tarsalis in foothills of Fresno County, California. Ann. Entomol. Soc. Am. 62: 13-18.

Kokernot, R.H., J. Hayes, N.J. Rose, and T.H. Work. 1967. St. Louis encephalitis in McLeansboro, Illinois, 1964. J. Med. Entomol. 4: 255-260.

Laws, E.R., F.R. Morales, W.R. Hayes, Jr., and C.R. Joseph. 1967. Toxicology of Abate in volunteers. Arch. Environ. Health 14: 289-291.

Leduc, J.W. 1979. The ecology of California group viruses. J. Med. Entomol. 16: 1-17.

LeDuc, J.E., W. Suyemoto, B.F. Eldridge, and E.S. Saugstad. 1972 . Ecology of arboviruses in a Maryland freshwater swamp. II. Blood feeding patterns of potential mosquito vectors. Am. J. Epidemiol. 96: 123-128.

Leiser, L.B., J.C. Beier, and G.B. Graig. 1982. The efficacy of malathion ULV spraying for urban Culex control in South Bend, Indiana. Mosquito News 43: 617-618.

Lemaitre, M.P. 1972. La premiere observation de case d'encephalite du type $E$ dans la province de Quebec. Revue M.V. Quebec 2: $33-36$. 
Leung, M.K., A. Burton, J. Iversen, and J. McLintock. 1975. Natural infection of Richardson's ground squirrels with western equine encephalitis virus, Saskatchewan, 1964-1973. Can. J. Microbiol. 21: 954-958.

Levin, S.A., K.D. Kimball, W.H. McDowe11, and S.F.Kimball. 1983. New perspectives in ecotoxicology. ERC Report 14a, Ecosystems Research Center, Cornell University. 158 pp.

Lillie, L.E., F.C. Wong, and R.A. Drysdale. 1976. Equine epizootic of western encephalomyelitis in Manitoba in 1975. Can. J. Pub1. H1 th. 67 (Suppl.): 21-27.

Lindsey, H.A., C.H. Calisher, and J.H. Mathews. 1976. Serum dilution neutralization test for California group virus identification and serology. J. Clin. Microbiol. 4: 503510 .

Lockhart, W.L., D.A. Metner, F.J. Ward, and G.M. Swanson. 1985. Population and cholinesterase responses in fish exposed to malathion sprays. Pest. Biochem. Physiol. 24: 12

Loborinsohn, M.E. 1987. Insecticide use and increased mortality in rural central Luzon, Phillipines. Lancet, June 13: 13591362 .

Luby, J.P. 1979. St. Louis Encephalitis. Epidemiol. Rev. 55 pp.

MacFarlane, B.L., J.A. Embil, H. Artsob, L. Spence, and K.R. Rozee. 1981. Antibodies to California group of arboviruses in the moose (Alces alces americana Clinton) population of Nova Scotia. Can. J. Microbiol. 27: 1219.

MacFarlane, B.L., J.E. Embree, J.A. Embril, K.R. Rozee, and H. Artsob. 1982. Antibodies to the California group of arboviruses in animal populations of New Brunswick. Can. J. Microbiol. 28: 200.

MacKay, J.F.W., W. Stackiw, and R.A. Brust. 1968. Western encephalitis (W.E.) in Manitoba - 1966. Man. Med. Rev. 2: $56-57$.

Mackenzie, D.L. 1979. Mosquito control in Ontario prior to, during and following the St. Louis encephalitis outbreak. In: Mhady, M.S., L. Spence, and J.M. Joshua (Eds.). Arboviral Encephalitides in Ontario With Special Reference to St. Louis Encephalitis. Ontario Ministry of Health. pp. 282-332. 
MacKenzie, D.L., H.E. Braun, and R. Frank. 1983. Municipal larviciding programs with temephos to control early and midseason mosquitoes in Ontario, 1974-80. Mosquito News 43: $187-194$.

Madder, D.J., R.S. MacDonald, G.A. Surgeoner, and B.V. Helson. 1980. The use of oviposition activity to monitor populations of Culex pipiens and Culex restuans (Diptera: Culicidae). Can. Ent. 112: 1013-1017.

Madder, D.J., G.A. Surgeoner, and B.V. Helson. 1983a. Induction of diapause in Culex pipiens and Culex restuans (Diptera:

Culicidae) in southern Ontario. Can. Ent. 115: 877-883.

Madder, D.J., G.A. Surgeoner, and B.V. Helson. 1983b. Number of generations, egg production, and developmental time of Culex pipiens and Culex restuans (Diptera: Culicidae) in southern Ontario. J. Med. Entomol. 20: 275-287.

Magnare11i, L.A. 1975. Relative abundance and parity of mosquitoes collected in dry-ice baited and unbaited CDC miniature light traps. Mosquito News 35: 350-353.

Mahdy, M.S., E. Bansen, and N.R. Paul. 1979a. Laboratory surveillance of acute human central nervous system disease in monitoring arbovirus activity in Ontario. In: Mahdy, M.S., L. Spence, and J.M. Joshua (Eds.). Arboviral Encephalitides in Ontario With Special Reference to St. Louis Encephalitis. Ontario Ministry of Health. pp. 244-281.

Mahdy, M.S., L. Spence, and J.M. Joshua (Eds.). 1979b. Arboviral Encephalitides in Ontario With Special Reference to St. Louis Encephalitis. In: The Committee on Programs for the Prevention of Mosquito-borne Encephalitis. Ontario Ministry of Health. xiii +364 pp.

Maibach, H.I., A.A. Khan, and R. Achilov, 1974 . Use of mosquito repellents for maximum efficiency. Arch. Dermato1. 109: 3235 .

Manitoba Clean Environment Commission. 1982. Report on mosquito control programs in Manitoba. 141 pp.

Manitoba Environment Workplace Safety and Health. 1984. Final Technical Report Volume. Environmental Monitoring Program for the 1983 Aerial Spraying of Malathion to Combat Western Equine Encephalitis. 225 pp. + appendices.

Marsha11, E. 1982. Fear as a form of pollution. Science 215: 481. 
Marshall, W.K., and J.R. Roberts. 1978. Ecotoxicology of chlorpyrifos. National Research Council of Canada, No. 16079. 314 pp.

Mayer, F.L., Jr., and M.R. Ellersieck. 1986. Manual of acute toxicity: interpretation and data base for 410 chemicals and 66 species of freshwater animals. U.S. Fish and Wildlife Service, Res. Pub. 160. 579 pp.

McDonald, P.T. 1975. Factors influencing diapause in Culex tarsalis. Proceedings and Papers of the Annual Conference of California Mosquito Control Association 43: 88.

McGregor, S.E. 1976. Insect pollination of cultivated crop plants. Agriculture Handbook No. 496. United States Department of Agriculture, Washington, D.C.

McGugan, A.C. 1941. Equine encephalomyelitis (Western type) in humans in Alberta, 1941. Can. Publ. H1th. J. 33: 148-151.

McLean, D.M. 1975. Arboviruses and human health in Canada. Associate Committee on Scientific Criteria for Environmental Quality. National Research Council of Canada, No. 14106. pp. $1-35$.

McLean, D.M. 1979. Arbovirus vectors in the Canadian Arctic. In: Kurstak, E. (Ed.). 1979. Arctic and Tropical Arboviruses. Academic Press, New York. 327 pp.

Mclean, D.M., S.K.A. Bergman, A.P. Gould, P.N. Grass, M.A. Miller, and E.E. Spratt. 1975. California encephalitis virus prevalence throughout the Yukon Territory, 1971-1974. Am. J. Trop. Med. Hyg. 24: 676-684.

McLean, D.M., S.K.A. Bergman, E.A. Graham, G.P. Greenfield, J.A. Olsen, and R.D. Patterson. 1974. California encephalitis virus presence in Yukon mosquitoes during 1973. Can. J. Publ. Hlth. 65: 23-28.

McLean, D.M., P.N. Grass, B.D. Judd, D. Omiralova, and K.M. Stuart. 1977. Natural foci of California encephalitis virus activity in the Yukon Territory. Can. J. Publ. H1th. 68: 6973 .

Mclean, D.M., S.M. Gubash, P.N. Gross, M.A. Miller, M. Petric, and T.E. Walters. 1975. California encephalitis virus development in mosquitoes as revealed by transmission studies, 
immunoperoxidase staining, and electron microscopy. Can. J. Microbiol. 21: 453-462.

McLintock, J. 1946. Collecting and handling mosquitoes on Western equine encephalitis investigations in Manitoba. Can. J. Res. E. 24: $55-62$

McLintock, J. 1947. Infection cycles in western equine and St. Louis encephalitis. Man. Med. Rev. No. 1047. pp. 635-637.

McLintock, J. 1948. Virus laboratory--Winnipeg. In: Dept. Publ. H1th. Ann. Rept., 1947. pp. 161-168.

McLintock, J. 1976. The arbovirus problem in Canada. Can. J . Pub1. Hlth. (Supp1. 1): 8-12.

McLintock, J. 1978. Mosquito-virus relationships of American encephalitides. Ann. Rev. Entomol. 23: 17-37.

McLintock, J., A.N. Burton, H. Dillenberg, and J.G. Rempel. 1966. Ecological factors in the 1963 outbreaks of western encephalitis in Saskatchewan. Can. J. Publ. Hlth. 57: 561575 .

McLintock, J., A.N. Burton, J.A. McKiel, R.R. Hall, and J.G. Rempe1. 1970. Known mosquito hosts of western encephalitis virus in Saskatchewan. J. Med. Entomol. 7: 446-454.

McLintock, J., P.S. Curry, R.J. Wagner, M.K. Leung, and J.0. Iversen. 1976. Isolation of Snowshoe Hare virus from Aedes implicatus larvae in Saskatchewan. Mosquito News 36: 233237.

McLintock, J., L.B. Hayles, and J.O. Iversen. 1979. Forecasting outbreaks of western equine encephalomyelitis in Saskatchewan. 21 pp. Unpublished Manuscript.

McLintock, J., and J. Iversen. 1975. Mosquitoes and human disease in Canada. Can. Ent. 107: 695-704.

McLintock, J., and J.G. Rempe1. 1963. Midsummer mosquito abundance in southern Saskatchewan, 1962. Mosquito News 23: 242-249.

Medovy, H. 1941. Western equine encephalomyelitis in infants. J. Ped. 22: 308-318.

Metcalf, R.L. 1973. A century of DDT. J. Agr. Food Chem. 21: 511519. 
Meyer, K.F., C.M. Haring, and B. Howitt. 1931. The etiology of epizootic encephalomyelitis in horses in the San Joaquin Valley, 1930. Science: 227-228.

Meyer, R.p., T. McKenzie, C.K. Fukushima, and R.K. Washino. 1975. Comparison of three sampling methods for measurement of adult mosquito populations. Proceedings and Papers of the Annual Conference of the California Mosquito Control Association 43: 154.

Milby, M.M., E.E. Kauffman, and J.F. Harvey. 1978. Conversion of CDC light trap indices to New Jersey light trap indices for several species of California mosquitoes. Proceedings and Papers of the Annual Conference of th California Mosquito and Vector Control Association 46: 58-60.

Milby, T.H., and W.L. Epstein. 1964. Allergic contact sensitivity to malathion. Arch. Environ. H1th. 9: 434-437.

Mitchell, C.A., and R.V.L. Walker. 1942. Studies in equine encephalitis suseptibility of some mammals and birds. Can. J. Comp. Med. 5: 314-319.

Mitche11, C.J. 1981. Diapause termination, gonoactivity, and differentiation of host-seeking behavior from blood-feeding behavior in hibernating Culex tarsalis (Diptera: Culicidae). J. Med. Entomol. 18: 386-394.

Mitche1l, C.J. 1979. Winter survival of Culex tarsalis (Diptera: Culicidae) hibernating in mine tunnels in Boulder County, Colorado, USA. J. Med. Entomol. 16:482-487.

Mitche11, C.J. 1983. Mosquito vector competence and arboviruses. In: Harris, K.F. (Ed.). Current Topics in Vector Research, Praeger Publishers, New York, N.Y. Pp. 63-92.

Mitche11, C.J., D.B. Francy, and T.P. Monath. 1980. Arthropod vectors. In: Monath, T.P., and W.C. Reeves (Eds.). St. Louis Encephalitis. American Public Health Association, Washington, D.C. Chapter 7. pp. 313-379.

Mitche11, C.J. 1983. Differentiation of host-seeking behaviour from blood-seeking behaviour in overwintering Culex pipiens (Diptera: Culicidae) and observations on gonotrophic dissociation. J. Med. Entomo1. 20: 157-163.

Monath, T.P. 1979. Arthropod-borne encephalitis in the Americas. 
Bu11. W.H.O. 57: 513-533.

Monath, T.P. (Ed.). 1980. St. Louis Encephalitis. American Public Health Association, Washington, D.C. $\mathrm{xx}+680 \mathrm{pp}$.

Monath, T.P. 1980. Epidemiology. In: Monath, T.P. (Ed.). 1980. St. Louis Encephalitis, American Public Health Association, Washington, D.C. pp. 239-312.

Monath, T.P. 1984. Ecology and control of mosquito-borne arbovirus disease. In: Kurstak, E., and R.G. Marusyk (Eds.). 1984. Control of Virus Diseases. Marcel Dekker, Inc., New York. pp. 115-134.

Monath, T.P., C.B. Cropp, G.S. Bowen, G.E. Kemp, G.E. Mitchell, and J.J. Gardner. 1980. Variation in virulence for mice and rhesus monkeys among $\mathrm{St}$. Louis encephalitis strains of different origin. Am. J. Trop. Med. Hyg. 29: 948-962.

Monath, T.P., G.E. Kemp, C.B. Cropp, and F.W. Chandler. 1978. Necrotizing myocarditis in mice infected with western equine encephalitis virus: clinical, electrocardiographic, and histopathologic correlations. J. Inf. Dis. 138: 59-66.

Moon, T.E. 1976. A statistical model of the dynamics of a mosquito vector (Culex tarsalis) population. Biometrics 32: 355-368.

Moore, C.G. 1963. Seasonal variation in autogeny in Culex tarsalis Coq. in Northern California. Mosquito News 23: 238-241.

Morgan, D.P., L.I. Lin, and H.H. Saikaly. 1980. Morbidity and mortality in workers occupationally exposed to pesticides. Arch. Environ. Contam. Toxicol. 9: 349-38

Morgante, O., E.M. Barager, and F.A. Herbert. 1968. Central nervous system disease in humans due to simultaneous epidemics of echovirus type 9 and western encephalomyelitis virus infection in Alberta. Can. Med. Assoc. J. 98: 11701175.

Morgante, O., and J.A. Shemanchuk. 1967. Virus of the California encephalitis complex: isolation from Culiseta inornata. Science 157: 692-693.

Morgante, O., H.N. Vance, J.A. Shemanchuck, and R. Windsor. 1968. Epizootic of Western Encephalomyelitis virus infection in equines in Alberta in 1965. Can. J. Comp. Med. 32:403-408. 
Mount, G.A., C.S. Lofgren, N.W. Pierce, and C.N. Husman. 1968. Ultra-low volume nonthermal aerosols of Malathion and Naled for adult mosquito control. Mosquito News 28: 99-103.

Mount, G.A., N.W. Pierce, and K.F. Baldwin. 1975a. Ultralow volume ground aerosols of propoxur (Baygon MOS) for control of adult mosquitoes. Mosquito News 35: 490-492.

Mount, G.A., N.W. Pierce, and K.F. Baldwin. 1975b. Comparison of two aerosol generator nozzle systems: estimates of droplet size and caged mosquito assays. Mosquito News 35: 501-504.

Mulder, D.W., M. Parrott, and M. Thaler. 1952. Sequelae of western equine encephalitis. Neurology 1: 318-327.

Nathanson, N. 1980. Pathogenesis. In: St. Louis Encephalitis. Monath, T.P. (Ed.) Chapt. 5. American Public Health Association, Washington, D.C. 1980. pp. 201-236.

National Research Council of Canada. 1982. Effects of propoxur on environmental quality with particular reference to its use for control of biting flies. No. 18572. $238 \mathrm{pp}$.

Nayer, J.K., L. Rosen, and J.W. Knight. 1986. Experimental vertical transmission of $\mathrm{St}$. Louis encephalitis virus by Florida mosquitoes. Am. J. Trop. Med. Hyg. 35: 1296-1301.

Nelson, R.L. 1964. Parity in winter populations of Culex tarsalis Coquillett in Kern County, California. Am. J. Hyg. 80: 242253.

Nelson, R.L., and R.D. Spadoni. 1972. Nightly patterns of biting activity and parous rates of some California mosquito species. Proceedings and Papers of the Annual Conference of the California Mosquito Control Association 40: 72-76.

Nelson, R.L., M.M. Milby, W.C. Reeves, and P.E.M. Fine. 1978. Estimates of survival, population size, and emergence of Culex tarsalis at an isolated site. Ann. Entomol. Soc. Am. 71: $801-808$.

Neufeld, J., and G. Nayar. 1982. Western equine encephalitis in Manitoba - equine cases 1975-1981. In: Sekla, L. (Ed.). Western equine encephalitis in Manitoba. Manitoba Health Services Commission. pp. 62-79.

Newhouse, V.F., R.W. Chamberlain, J. Johnston, and D.W. Sudia . 1966. Use of dry ice to increase mosquito catches of the CDC 
miniature light trap. Mosquito News 26: 30-35.

Norris, M. 1946. Recovery of a strain of western equine encephalitis virus from Culex restuans. Can. J. Res. E. 24: $63-70$.

Olson, J.G., W.C. Reeves, R.W. Emmons, and M.M. Milby. 1979. Correlation of Culex tarsalis population indices with the incidence of St. Louis encephalitis and western equine encephalomyelitis in California. Am. J. Trop. Med. Hyg. 28: $335-343$.

Panetta, J. (Ed.). 1980. DEET pesticide registration standard. U.S. EPA, Office of Pesticides and Toxic Substances, 136 pP.

Pavilanis, V., I.L. Wright, and M. Silverberg. 1957. Western equine encephalitis: report of a case in Montreal. Can. Med. Assoc. J. 77: 128-130.

Pearce, P.A., L.M. Reynolds, and D.B. Peaka11. 1978. DDT residues in rainwater in New Brunswick and estimate of aerial transport of DDT into the Gulf of St. Lawrence, 1967-68. Pestic. Monit. J. 11: 199-204.

Petersen, J.J., and O.R. Willis. 1972. Procedures for the mass rearing of a mermithid parasite of mosquitoes. Mosquito News 32: $226-230$.

Phillips, L., M. Steinberg, H.I. Maibach, and W.A. Akers. 1972. A comparison of rabbit and human skin response to certain irritants. Toxicol. Appl. Pharmacol. 21: 369-382.

Pinger, R.R., W.A. Rowley, Y.W. Wong, and D.C. Dorsey. 1975. Trivittatus virus infections in wild mammals and sentinel rabbits in central Iowa. Am. J. Trop. Med. Hyg. 24: 10061009 .

Prior, M.G., and R.M. Agnew. 1971. Antibody against western equine encephalitis virus occurring in the serum of garter snakes (Colubridae: Thamnophis) in Saskatchewan. Can. J. Comp. Med. 35: 40-43.

Quong, T.L. 1942. The pathology of western equine encephalomyelitis. 18 human cases, Manitoba epidemic, 1941. Can. Pub. Hlth. J. 33: 300-306.

Raddatz, R.I. 1982. Forecasts of Culex tarsalis populations in Winnipeg. In: Sekla, L. (Ed.) Western Equine Encephalitis in 
Manitoba. Manitoba Health Services Commission. 296 pp.

Raddatz, R.L. 1985. A biometeorological model of an encephalitis vector. Boundary Layer Meteorol. 34: 185-199.

Rainey, M.B., G.V. Warren, A.D. Hess, and J.S. Blackmore. 1962. A sentinel chicken shed and mosquito trap for use in encephalitis field studies. Mosquito News 22: 337-342.

Randall, A.P. 1977. Research developments in aerial application techniques for spruce budworm control in the province of Quebec (with reference to fenitrothion). In: Roberts, J.R., R. Greenhalgh, and W.K. Marshall (Eds.). Proceedings of a Symposium on Fenitrothion: the long term effects of its use in forest ecosystems. National Research Council, Ottawa. pp. $159-182$.

Rapport, D.A., H.A. Regier, and T.C. Hutchinson. 1985. Ecosystem behavior under stress. Am. Nat. 125: 617-640.

Rathburn, C.B., Jr., and A.H. Boike, Jr. 1975. Ultra low volume tests of several insecticides applied by ground equipment for the control of adult mosquitoes. Mosquito News 35: 26-29.

Rathburn, C.B., Jr., A.H. Boike, C.F. Hallman, and R.L. Welles. 1981. Field tests of insecticides applied as ULV sprays by ground equipment for the control of adult mosquitoes.

Mosquito News 41: 132-135.

Rees, D.M., L.J. Ogden, G.C. Collett, and J.E. Graham. 1959. The 1958 encephalitis outbreak in Northern Utah. 3. Mosquito populations in relation to the outbreak. Mosquito News 19: $227-231$.

Reeves, W.C. 1965. Ecology of mosquitoes in relation to arboviruses. Ann. Rev. Entomol. 10: 25-46.

Reeves, W.C. 1968. A review of developments associated with the control of Western Equine and St. Louis Encephalitis in California during 1967. Proceedings and Papers of the Annual Conference of the California Mosquito Control Association 36: $65-70$.

Reeves, W.C. 1971. Mosquito vector and vertebrate host interaction: the key to maintenance of certain arboviruses. In: Fallis, A.M. (Ed.). Ecology and Physiology of Parasites. University of Toronto Press, Toronto. 
Reeves, W.C. 1974. Overwintering of arboviruses. 1974. Progr. Med. Virol. 17: 193-220.

Reeves, W.C., R.E. Bellamy, A.F. Geib, and R.P. Scrivani. 1964. Analysis of the circumstances leading to abortion of a western equine encephalitis epidemic. Am. J. Hyg. 80: 205220 .

Reeves, W.C., R.E. Bellamy, and R.P. Scrivani. 1961.

Differentiation of encephalitis virus infection rates from transmission rates in mosquito vector populations. Am. J. Hyg. 73: $303-315$.

Reeves, W.C., and W. McD. Hammon. 1952. California encephalitis virus, a newly described agent. III. Mosquito infection and transmission. J. Immunol. 69: 511-514.

Reeves, W.C., and W. McD. Hammon. 1962. Epidemiology of the arthropod-borne viral encephalitides in Kern County, California, 1943-1952. University of California Press, Berkeley. 257 pp.

Reeves, W.C., W. McD. Hammon, D.P. Furman, H.E. McClure, and B. Brookman. 1947. Recovery of western equine encephalitis virus from wild bird mites (Liponyssus sylvarium) in Kern County, California. Science 105: 411.

Reisen, W.K. 1986. Overwintering studies on Culex tarsalis (Diptera: Culicidae) in Kern County, California: life stages sensitive to diapause induction cues. Ann. Entomol. Soc. Am. 79: $674-676$.

Reisen, W.K., R.P. Meyer, and M. M. Milby. 1986a. Overwintering studies on Culex tarsalis (Diptera: Culicidae) in Kern County, California: survival and experimental induction and termination of diapause. Ann. Entomol. Soc. Am. 79: 664673.

Reisen, W.K., R.P. Meyer, and M.M. Milby. 1986b. Overwintering studies on Culex tarsalis (Diptera: Culicidae) in Kern County, California: temporal changes in abundance and reproductive status with comparative observations on $C$. quinquefasciatus (Diptera: Culicidae). Ann. Entomol. Soc. Am. 79: 677-685.

Reisen, W., M. Milby, W. Reeves, R. Meyer, and M. Block. 1983. Population ecology of Culex tarsalis (Diptera: Culicidae) in a foothill environment of Kern County, California: temporal 
changes in female relative abundance, reproductive status and survivorship. Ann. Entomol. Soc. Am. 76: 800-808.

Reisen W.K. and T. P. Monath, 1989. Western Equine

Encephalomyelitis. In: Monath, T.P. (Ed.). The Arboviruses: Epidemiology and Ecology. Vol. V. pp. 89-137.

Reisen, W.K., G. Yoshimura, W.C. Reeves, M.M. Milby, and R.P. Meyer. 1984. The impact of aerial applications of ultra-low volume adulticides on Culex tarsalis populations (Diptera: Culicidae) in Kern County, California, U.S.A. 1982. J. Med. Entomol. 21: 573-585.

Reiter, P. 1983. A portable, battery-powered trap for collecting gravid Culex mosquitoes. Mosquito News 43: 496-498.

Ripper, W.E. 1956. Effect of pesticides on balance of arthropod populations. Ann. Rev. Entomol. 1: 403-438.

Ritter, D.G., and E.T. Feltz. 1974. On the natural occurrence of California encephalitis virus and other arboviruses in Alaska. Comp. J. Microbiol. 20: 1359-1366.

Roehrig, J.T., J.H. Mathews, and D.W. Trent. 1983. Identification of epitopes on the $\mathrm{E}$ glycoprotein of St. Louis encephalitis virus using monoclonal antibodies. Virology 128: 118-126.

Rodzilsky, B., H.E. Robertson, and J. Chorney. 1968. Western encephalitis: report of eight fatal cases: Saskatchewan epidemic, 1965. Can. Med. Assoc. J. 98: 79-86.

Rosen, L. 1987. Overwintering mechanisms of mosquito-borne arboviruses in temperate climates. Am. J. Trop. Med. Hyg. 37 (Supp1.): $69-76$.

Rowley, W.A., Y.W. Wong, R.W. Currier, D.C. Dorsey, B.M. Christensen, and W.J. Hausler, Jr. 1979. Mosquito-borne virus activity in Iowa, 1975-1976. Mosquito News 39: 1-9.

Rush, W.A. 1962. Observations on an overwintering population of Culex tarsalis with notes on other species. Mosquito News. 22: $176-181$.

Savage, A. 1942. Note on the prevalence of encephalomyelitis in Manitoba horses prior to 1941. Can. Publ. H1th. J. 33: 258.

Schaefer, C.H., and T. Miura. 1972. Source of energy utilized by natural populations of the mosquito Culex tarsalis, for 
overwintering. J. Insect Physiol. 18: 797-805.

Schaefer, C.H. T. Miura, and R.K. Washino. 1971. Studies on the overwintering biology of natural populations of Anopheles freeborni and Culex tarsalis in California. Mosquito News 31: $153-157$.

Schaefer, C.H., and R.K. Washino. 1969. Changes in the composition of lipids and fatty acids in adult Culex tarsalis and Anopheles freeborni during the overwintering period. J. Insect Physiol. 15: 395-402.

Schaefer, C.H., and R.K. Washino. 1970. Synthesis of energy for overwintering in natural populations of the mosquito $\mathrm{Culex}$ tarsalis. Comp. Biochem. Physiol. 35: 503-506.

Schiefer, B.A., R.W. Vavra, Jr., R.L. Frommer, and E.J. Gerber. 1976. Field evaluation of several repellents against black flies (Diptera, Simuliidae). Mosquito News 36: 242-247.

Sciple, G.W., C.G. Roy, P. Holden, L.C. LaMotte, J.V. Irons, and T.D.Y. Chin. 1968. Encephalitis in the high plains of Texas. Am. J. Epidemiol. 87: 87-98.

Sekla, L.H. (Ed.). 1976. Special Supplement Western Encephalitis. Can. J. Publ. H1th. 67 (Supp1.). 75 pp.

Sekla, L. (Ed.). 1982. Western equine encephalitis in Manitoba. Manitoba Health Services Commision. 296 pp.

Sekla, L.H., and W. Stackiw. 1976. VI. Laboratory diagnosis of western encephalomyelitis. Can. J. Publ. Hlth. 67 (Supp1.): $33-39$.

Sekla, L.H., and W. Stackiw. 1982. Arbovirus isolations and serology in Manitoba. In: Sekla, L. (Ed.). Western Equine Encephalitis in Manitoba. Manitoba Public Services Commission. pp. 103-116.

Sekla, L., and W. Stackiw. 1982. Arbovirus isolations from mosquitoes in Manitoba: value in decision making. In:

Sekla, L. (Ed.). Western Equine Encephalitis in Manitoba, Manitoba Health Services Commission. pp. 50-60.

Sekla, L., W. Stackiw, and R.A. Brust. 1980. Arbovirus isolations from mosquitoes in Manitoba. Mosquito News 49: 377-380.

Service, M.W. 1976. Mosquito Ecology. Field Sampling Methods. 
John Wiley and Sons. New York, Toronto. xii +583 pp.

Seymour, K.G. 1969. Evaluation of spray drift potential. In: Gould, R.F. (Ed.). Pesticidal formulations research, physical and colloidal chemical aspects. Adv. Chem. Ser., Amer. Chem. Soc. $86: 135-154$.

Sheehan, P.J., A. Baril, P. Mineau, D.K. Smith, A. Harfenist, and W.K. Marshall. 1987. The impact of pesticides on the ecology of prairie nesting ducks. Can. Wildl. Serv., Tech. Rept. Ser. 19 .

Shelton, R.M. 1973. The effect of temperature on development of eight mosquito species. Mosquito News 33: 1-12.

Shemanchuk, J.A. 1969a. Epidemiology of western encephalitis in Alberta: response of natural populations of mosquitoes to avian host. J. Med. Entomol. 6: 269-275.

Shemanchuk, J.A. 1969b. On the hibernation of Culex tarsalis Coquillett, Culiseta inornata Williston, and Anopheles earlei Vargas (Diptera: Culicidae) in Alberta. Mosquito News 25: 456-462.

Shemanchuk, J.A., and O. Morgante. 1968. Isolation of western encephalitis virus from mosquitoes in Alberta. Can. J. Microbio1. 14: 1-5.

Slovic, P. 1987. Perception of risk. Science 236: 280-285.

Smart, K.L., R.E. Elbel, R.F.N. Woo, E.R. Kern, G.T. Crane, G.L. Bales, and D.W. Hil1. 1972. California and western encephalitis viruses from Bonneville Basin, Utah in 1965. Mosquito News 32: 382-389.

Snel1, E. 1966. Preventative medicine - selective topics of current interest. Man. Med. Rev. 46: 23-29.

Spadoni, R.D., R.L. Nelson, and W.C. Reeves. 1975. Seasonal occurrence, egg production, and blood-feeding activity of autogenous Culex tarsalis. Ann. Ent. Soc. Am. 67: 895-902.

Spalatin, J., A.N. Burton, J. McLintock, and R. Connell. 1963. Isolation of western equine encephalitis (WEE) virus from mosquitoes in Saskatchewan, 1962. Can. J. Comp. Med. Vet. Sci. 27: 283-289. 
Spalatin, J., R. Corne11, A.N. Burton, and B.J. Gallop. 1964. Western equine encephalitis in Saskatchewan reptiles and amphibians, 1961-1963. Can. J. Comp. Med. Vet. Sci. 28: 131142 .

Spence, I., Artsob, H., Grant, L., and C. Th'ng. 1977. St. Louis encephalitis in southern Ontario: laboratory studies for arboviruses. Can. Med. Assoc. J. 116: 35-36.

Spencer, T.S., J.A. Hill, R.J. Feldman, and H.I. Maibach. 1979. Evaporation of diethyl toluamide from human skin. J. Investigative Dermato1. 72: 317-319.Sulkin, S.E., and R. Allen. 1974. Virus infection in bats. Mongr. Virol. Vol. 8. 103 pp.

Spielman, A. 1971a. Studies on autogeny in natural populations of Culex pipiens, its seasonal abundance of autogenous and anautogenous populations. J. Med. Entomo1. 8: 555-561.

Spielman, A. 1971b. Bionomics of autogenous mosquitoes. Ann. Rev. Entomo1. 16: 231-248.

Spielman, A., and J. Wong. 1973a. Studies on autogeny in natural populations of Culex pipiens. III. Mid-summer preparation for hibernation in autogenous populations. J. Med. Entomol. 10: $319-324$.

Spielman, A., and J. Wong. 1973b. Environmental control of ovarian diapause in Culex pipiens. Ann. Entomol. Soc. Am. 66: 905907.

Srihongse, S., J.P. Woodal1, M.A. Grayron, R. Deibel, T.F. Bast, C.D. Morris, E.M. Bosler, J.L. Benach, J.J. Howard, and J. Berlin. 1980. Arboviruses in New York State: surveillance in arthropods and nonhuman vertebrates, 1972-1977. Mosquito News 40: 269-276.

Strickman, D. 1979. Malathion as ground applied ULV evaluated against natural populations of Culex pipiens and $C x$. restuans. Mosquito News 39: 64-67.

Sudia, W.D., and R.W. Chamberlain. 1962. Battery-operated light trap, an improved model. Mosquito News 22: 126-129.

Sulkin, S.E., and R. Allen. 1974. Virus infection in bats. Mongr. Virol. Vo1. 8. $103 \mathrm{pp}$. 
Surgeoner, G.A., W. Ralley, and M. Mahdy. 1982. Investigation of western equine encephalitis activites in north western Ontario 1981. In: Sekla, L. (Ed.). Western Equine Encephalitis in Manitoba. Manitoba Health Services Commission. pp. 80-84.

Surgeoner, G.A., and B.V. Helson. 1978. An oviposition trap for arbovirus surveillance in Culex sp. mosquitoes (Diptera: Culicidae). Can. Ent. 110: 1049-1052.

Suzuki, M., and C.A. Phillips. 1966. St. Louis encephalitis, histopathologic study of fatal cases from Houston epidemic in 1964. Arch. Pathol. 81: 47.

Takata, N., and R.F. Harwood. 1964. Fatty acid composition during postembryonic development of the mosquito Culex tarsalis. Ann. Entomol. Soc. Am. 57: 749-753.

Taylor, R.G., and H.F. Schoof. 1971. The relative effectiveness of malathion thermal aerosols and ground-applied ULV against three species of mosquitoes. Mosquito News 31: 346-349.

Tekle, A. 1960. The physiology of hibernation and its role in the geographical distribution of populations of the Culex pipiens complex. Am. J. Trop. Med. Hyg. 9: 321-330.

Tempelis, C.H. 1975. Host-feeding patterns of mosquitoes, with a review of advances in analysis of blood meals by serology. J. Med. Entomo1. 11: 635-653.

Tempelis, C.H., W.C. Reeves, R.E. Bellamy, and M.F. Lofy. 1965. A three-year study of the feeding habits of Culex tarsalis in Kern County, California. Am. J. Trop. Med. Hyg. 14: 170177.

Tempelis, C.H., and R.K. Washino. 1967. Host-feeding patterns of Culex tarsalis in the Sacramento Valley, California, with notes on other species. J. Med. Entomo1. 4: 315-318.

Tesh, R.P. 1984. Transovarial transmission of arboviruses in their invertebrate vectors. In: Harris, K.F. (Ed.). Current Topics in Vector Research. Vol. II. Chapter 3. pp. 57-76.

Thorsen, J., H. Artsob, L. Spence, G. Surgeoner, B. Helson, and R. Wright. 1980. Virus isolations from mosquitoes in southern Ontario, 1976 and 1977. Can. J. Microbiol. 26: 436-440. 
Thorsen, J., H. Artsob, G. Surgeoner, B. Helson, L. Spence, and R. Wright. 1979. Virus isolations from mosquitoes in southern Ontario 1976 and 1977. In: Mahdy, M.S., L. Spence, and J.M. Joshua (Eds.). Arboviral Encephalitides in Ontario With Special Reference to St. Louis Encephalitis. Ontario Ministry of Health. pp.199-207.

Trainer, D.O., and G.L. Hoff. 1971. Serologic evidence of arbovirus activity in a moose population in Alberta. J. Wildl. Dis. 7: 118-119.

Trent, D.W., T.P. Monath, G.S. Bowen, A.V. Vorden, C.B. Cropp, and G.R. Kemp. 1980. Variation among strains of St. Louis encephalitis virus: basis for genetic pathogenic, and epidemiologic classification. Ann. N.Y. Acad. Sci. 354: 219237.

Tsai, T.F., and C.J. Mitche11. 1989. St. Louis Encephalitis. In: Monath, T.P. (Ed.), The Arboviruses: Epidemiology and Ecology. Vol. IV. PP. 113-143.

Turel1, M.J., and J.W. LeDuc. 1983. The role of mosquitoes in the natural history of California serogroup viruses. In:

Calisher, C.H., and W.H. Thompson (Eds.). California Group Viruses. pp. 43-55.

Walker, T., and M.V. Meisch. 1982. Evaluation of pyrethrum and two synthetic pyrethroids alone and in mixtures with malathion and ULV ground aerosols against riceland mosquitoes. Mosquito News 42: 167-171.

Walsh, J.D. (Ed.) 1987. California's mosquitoborne encephalitis virus surveillance and control program. California Department of Health Services, Vector Surveillance and Control Branch. $46 \mathrm{pp}$.

Walton, T.E. 1921. Venezuelan, Eastern and Western Encephalomyelitis. In: Gibbs, E.P.J. (Ed.) Virus Diseases of Food Animals. Academic Press. New York. Pp. 587-625.

Ward, D.V., B.L. Howes, and D.F. Ludwig. 1976. Interactive effects of predation pressure and insecticide (Temephos) toxicity on populations of the marsh fiddler crab Uca pugnax. Mar. Biol. 35: $116-126$.

Ware, G.W., N.A. Buck, and B.J. Estesen. 1984. Deposit and drift losses from aerial ultra-low-volume and emulsion sprays in Arizona. J. Econ. Entomol. 77: 298-303. 
Waters, J. 1976. An epidemic of western encephalomyelitis in humans-Mani toba 1975. Can. J. Publ. Hlth. 67(Supp.): 28-32.

Watts, D.M., G.G. Clark., C.L. Crabbs, C.A. Rossi, T.R. Ollin, and C.L. Bailey. 1987. Ecological evidence against vertical transmission of eastern equine encephalitis virus by mosquitoes (Diptera: Culicidae) on the Delmarva Peninsula, U.S.A. J. Med. Entomo1. 24: 91-98.

Watts, D.M., G.R. DeFoliart, and T.M. Yuill. 1976. Experimental transmission of trivittatus virus (California virus group) by Aedes trivittatus. Am. J. Trop. Med. Hyg. 25: 173-176.

Watts, D.M., and B.F. Eldridge. 1975. Transovarial transmission of arboviruses by mosquitoes: a review. Med. Biol. 53: 271278 .

Wheatley, G.A. 1973. Pesticides in the atmosphere. In: Edwards, C.A. (Ed.). Environmental Pollution By Pesticides. Plenum Press. pp. 365-408.

Wilton, D.P., and G.C. Smith. 1985. Ovarian diapause in the geographic strains of Culex pipiens (Diptera: Culicidae). J. Med. Entomol. 22: 524-528.

Womeldorf, J.D., E.E. Lusk, K.R. Townzen, and P.A. Gillies. 1973. Evaluation of low volume nonthermal aerosols for mosquito control in California. Proceedings of the California Mosquito Control Association 42: 69-73.

Wong, T.C., L.E. Lillie, and R.A. Drysdale. 1976. Sentinel flock monitoring procedures for Western Encephalomyelitis in Manitoba-1975. Can. J. Publ. Hlth.67 (Supp1.): 15-20.

Wong, F., and J. Neufeld. 1982. Sentinel flock monitoring procedures for Western Equine Encephalitis in Manitoba, 19761981. In: Sekla, L. (Ed.). 1982. Western Equine Encephalitis in Manitoba, Manitoba Health Services Commission. pp. 86-97.

Wood, D.M., P.T. Dang, and R.A. Ellis. 1979. The insects and arachnids of Canada, (Diptera: Culicidae). Canadian Government Publishing Centre, Supply and Services Canada, Hull, Quebec. 390 pp.

Yuill, T.M., and R.P. Hanson. 1964. Serologic evidence of California encephalitis virus and western equine encephalitis 
virus in snowshoe hares. Zoon. Res. 3: 153-164.

Yuill, T.M., J.O. Iverson, and R.P. Hanson. 1969. Evidence for arbovirus infections in a population of snowshoe hares: a possible mortality factor. Bull. Wildl. Dis. Assoc. 5: 248253.

Zink1, J.G., J. Rathert, and R.R. Hudson. 1978. Diazinon poisoning in wild Canada geese. J. Wildl. Manag. 42: 406-408.

Zweighaft, R.M., C. Rasmussen, A. Brolnitsky, and J.C. Lashof. 1979. St. Louis encephalitis: the Chicago experience. Am. J. Trop. Med. Hyg. 28: 114-118. 
INSECTICIDES COMMONLY USED IN MOSQUITO CONTROL PROGRAMS IN CANADA

This appendix provides information (1) on the insecticides that are commonly used in municipal mosquito abatement programs in Canada, and (2) on the repellents available for personal use and for use on livestock to protect against mosquito bites.

Although there are several hundred pest control products registered for use against mosquitoes in Canada, most of these products are for the home-owner. Relatively few insecticides are used in routine mosquito abatement programs. The products listed below have been extracted from an all-inclusive listing provided by the Pesticides Directorate of Agriculture Canada. They are only examples of those that have been or are being used for largescale mosquito control programs. A complete pest control product listing is beyond the scope of this manual.

The 3-letter code at the beginning of each line refers to the registrant. The names and addresses of these registrants are given in Appendix 3. The number following each registrant's code is the Pest Control Products Act registration number.

TEMEPHOS

$\begin{array}{lll}\text { KEM } & 13694.00 & \text { RIDDEX ABATE 4E } \\ \text { KEM } & 13695.00 & \text { KEMSAN ABATE 2-G } \\ \text { SAF } & 15567.00 & \text { SANEX ABATE 2G } \\ \text { SAF } & 16460.00 & \text { SANEX ABATE 4E }\end{array}$

PROPOXUR

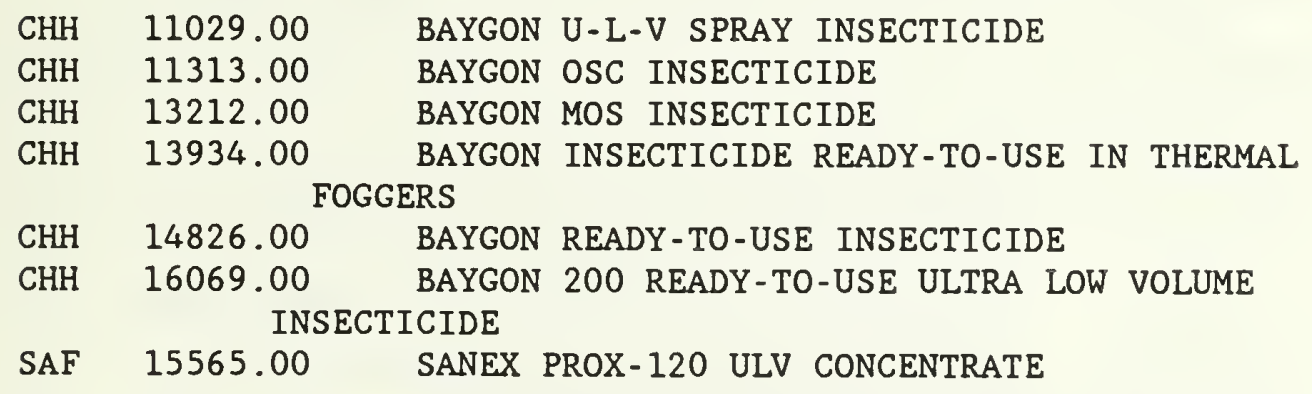

BACILLUS THURINGIENSIS ISRAELENSIS (BTI)

$\begin{array}{llll}\text { ABT } & 18158.00 & \text { VECTOBAC - 200 G } \\ \text { ABT } & 19455.00 & \text { VECTOBAC 600 L } \\ \text { ABT } & 19466.00 & \text { VECTOBAC 200 G } \\ \text { DUP } & 19220.00 & \text { BACTIMOS G }\end{array}$




\begin{tabular}{lr} 
ZOE & \multicolumn{1}{r}{19241.00} \\
ZOE & 19239.00 \\
& \multicolumn{2}{l}{} \\
CHLORPYRIFOS \\
DOW & \multicolumn{1}{l}{10636.00} \\
DOW & 10637.00 \\
DOW & 12223.00 \\
& \multicolumn{1}{l}{} \\
MALATHION \\
CGC & 12590.00 \\
CHP & 11591.00 \\
CYC & 9337.00 \\
& \\
DIS & 13883.00 \\
DIT & 9975.00 \\
EMO & 18322.00 \\
GAX & 8624.00 \\
GAX & 16198.00 \\
GRA & 15380.00 \\
INT & 5821.00 \\
INT & 8480.00 \\
KEK & 18184.00 \\
KEM & 9920.00 \\
& \\
KEM & 12216.00 \\
KEM & 15994.00 \\
SAF & 6022.00 \\
SAF & 9947.00 \\
SAF & 16099.00 \\
UAG & 19364.00 \\
&
\end{tabular}

METHOXYCHLOR

$\begin{array}{ll}\text { CGC } & 10481.00 \\ \text { CHP } & 10603.00 \\ & \\ \text { GRA } & 15382.00 \\ \text { GRX } & 16208.00 \\ \text { INT } & 13558.00 \\ \text { INT } & 18088.00 \\ \text { KEM } & 19172.00 \\ \text { LAT } & 10690.00 \\ \text { MBY } & 14772.00 \\ \text { SAF } & 12733.00 \\ \text { STD } & 11617.00 \\ \text { UAG } & 13727.00\end{array}$

TEKNAR HP-D

TEKNAR G
DURSBAN*2E

DURSBAN*4E

DURSBAN $2 \quad 1 / 2 \quad G$

\author{
MALATHION 500 EC \\ CHIPMAN MALATHION 500 \\ CYTHION LIQUID INSECTICIDE PREMIUM GRADE \\ MALATHION \\ MALATHION 50
}

MALATHION 5OE EMULSIFIABLE LIQUID INSECTICIDE M-50 EMULSIFIABLE CONCENTRATE

GARDEX 50\% MALATHION EC INSECTICIDE

GARDEX MALATHION ULV CONCENTRATE

GREEN LEAF MALATHION 50\% EC INSECTICIDE

CO-OP MALATHION 500 EC INSECTICIDE

CO-OP MALATHION INSECT SPRAY

VANQUISH EMULSIFIABLE CONCENTRATE

RIDDEX MALATHION 500 EC EMULSIFIABLE

CONCENTRATE

RIDDEX CYTHION ULV INSECTICIDE

RIDDEX MALFOG 45 FOGGING INSECTICIDE

SANEX MALATHION 5OM

SANEX DYNA-FOG M-L LIQUID INSECTICIDE

SANEX MALATHION 50 EC

CLEAN CROP MALATHION 50\% EC INSECTICIDE
METHOXYCHLOR 240 EC

CHIPMAN METHOXYCHLOR SPRAY CONCENTRATE

INSECTICIDE

GREEN LEAF METHOXYCHLOR 25\% EC INSECTICIDE METHOXYCHLOR 240

CO-OP METHOXYCHLOR 25 EC INSECTICIDE

IPCO METHOXYCHLOR 25\% EC

KEMSAN METHOXYCHLOR 240 EC INSECTICIDE

LATER'S METHOXYCHLOR 25\% EC INSECTICIDE

METHOXOL 240-EC

SANEX MOXY GARDEN SPRAY CONCENTRATE

METHOXYCHLOR 2.4 EC INSECTICIDE

CLEAN CROP METHOXYCHLOR 240 
METHOPRENE

ZOE $\quad 13797.00$

ALTOSID SR-10 INSECT GROWTH REGULATOR

ZOE $\quad 15152.00$

ALTOSID BRIQUET MOSQUITO GROWTH REGULATOR

PYRETHRINS

$\mathrm{ABE} \quad 9836.00$

WACO TOSSIT PYRETHRUM FORMULA TO KILL MOSQUITO LARVAE

RESMETHRIN

PEI $\quad 15187.00$

SBP-1382 MOSQUITO FOG - 40\%

2. REPELLENTS FOR PROTECTION OF PEOPLE \& LIVESTOCK

Several hundred repellents are registered in Canada for protecting humans, pets and livestock (including horses) from mosquito bites. Available space does not permit the listing of all such pest control products. The presence or absence of a product on this listing does not constitute endorsement or otherwise. The examples of repellents given below are used only to illustrate the active ingredients, types of formulations, target users, and manufacturers involved.

The repellents listed are sorted by active ingredient. The 3-letter code at the beginning of each line signifies the registrant. The number following the 3 -letter code is the Pest Control Products registration number. Some repellents are listed under several active ingredients because the formulations contain 2 or more active ingredients. The names and addresses of the registrants are given in Appendix III.

OIL OF CITRONELLA

DTC $\quad 8719.00$

CERTIFIED CITRONELLA OIL INSECT REPELLENT

MCO $\quad 1526.00$ REPALFLY MCKIRDY'S SPECIAL FLY REPELLENT

MRA 18500.00

$X$-IT INSECT REPELLENT

MRA 20779.00

$\mathrm{X}$-IT INSECT REPELLENT STICK

NEW $\quad 18682.00$

V-TO ROLL ON CITRONEL MILK

NEW $\quad 18780.00$

V-TO PUMP SPRAY CITRONEL MILK 


$\begin{array}{lrl}\text { JBL } & 15433.00 & \text { BF-100 BLACK FLY REPELLENT } \\ \text { MCO } & 1526.00 & \text { REPALFLY MCKIRDY'S SPECIAL FLY REPELLENT } \\ \text { MLS } & 18364.00 & \text { CUTTER EVERGREEN SCENT INSECT REPELLENT } \\ \text { MLS } & 18365.00 & \text { CUTTER INSECT REPELIENT } \\ \text { MLS } & 18366.00 & \text { CUTTER EVERGREEN SCENT INSECT REPELLENT CREAM } \\ \text { MRA } & 20779.00 & \text { X-IT INSECT REPELLENT STICK } \\ \text { NOZ } & 12401.00 & \text { NOXEMA INSECT REPELLENT LOTION } \\ \text { NOZ } & 12402.00 & \text { NOXEMA TAN \& GUARD LOTION }\end{array}$

DEET PLUS RELATED ACTIVE TOLUAMIDES

$\begin{array}{lrll}\text { AFY } & 20846.00 & \text { SKEETER BEATER INSECT REPELLENT } \\ \text { AIG } & 14333.00 & \text { AIR GUARD INSECT REPELLENT BUSH STRENGTH } \\ \text { ANW } & 9154.00 & \text { AMWAY D-15 INSECT REPELLENT } \\ \text { AMW } & 14811.00 & \text { AMWAY D-15 INSECT REPELLENT TOWELETTE } \\ \text { ARE } & 17746.00 & \text { UNCLE KEITH'S BUG FREE INSECT REPELLENT } \\ \text { ARE } & 18726.00 & \text { UNCLE KEITH'S BUG FREE JACKET } \\ \text { ARI } & 19018.00 & \text { DEET-1OO INSECT REPELLENT } \\ \text { AVM } & 16638.00 & \text { EN-GARDE LONG LASTING INSECT REPELLENT } \\ \text { BDC } & 18399.00 & \text { BRENTDALE CHEMICALS INSECT REPELLENT } \\ \text { BOY } & 17956.00 & \text { COMBAT INSECT REPELLENT } \\ \text { BOY } & 17957.00 & \text { COMBAT LIQUID INSECT REPELLENT } \\ \text { CCN } & 18040.00 & \text { CONN CHEM INSECT REPELLENT } \\ \text { CCN } & 20774.00 & \text { CCL QUICK-BREAKING INSECT REPELLENT FOAM II } \\ \text { CGC } & 4039.00 & \text { TANTOO REPELLENT } \\ \text { CGC } & 12958.00 & \text { TANTOO GEL } \\ \text { CHZ } & 16811.00 & \text { COGHLANS INSECT REPELLENT } \\ \text { COS } & 18400.00 & \text { COPELAND INSECT REPELLENT PRESSURIZED SPRAY } \\ \text { DEV } & 16818.00 & \text { DECA INSECT REPELLENT } \\ \text { DFT } & 19107.00 & \text { THE ORIGINAL SKEETO-BAN JACKET OR PANTS } \\ \text { DFT } & 19194.00 & \text { THE ORIGINAL SKEETO-BAN INSECT REPELLENT } \\ \text { FFL } & 18419.00 & \text { BUG STOP 1OO } \\ \text { FUB } & 10790.00 & \text { FULLER REPEL-GEL } \\ \text { GUC } & 18199.00 & \text { GOTCHA! INSECT REPELLENT } \\ \text { HOH } & 18499.00 & \text { RIVER TRAIL LIQUID INSECT REPELLENT } \\ \text { INT } & 9601.00 & \text { CO-OP INSECT REPELLENT } \\ \text { INT } & 19772.00 & \text { CO-OP INSECT REPELLENT PRESSURIZED SPRAY } \\ \text { JOH } & 9203.00 & \text { OFF! FRESH OUTDOOR SCENT INSECT REPELLENT } \\ \text { JOH } & 11004.00 & \text { OFF! INSECT REPELLENT } \\ \text { JOH } & 13252.00 & \text { DEEP WOODS FORMULA OFF INSECT REPELLENT LOTION } \\ \text { JOH } & 13253.00 & \text { DEEP WOODS PRESSURIZED SPRAY INSECT REPELLENT } \\ \text { JOH } & 13990.00 & \text { OFF! INSECT REPELLENT TOWELETTES } \\ \text { JOH } & 15583.00 & \text { OFF! PUMP SPRAY INSECT REPELLENT } \\ \text { JOH } & 18896.00 & \text { DEEP WOODS OFF! PUMP LIQUID } \\ \text { JOH } & 19154.00 & \text { DEEP WOODS MAXIMUM STRENGTH SPRAY } \\ & & \end{array}$




\begin{tabular}{|c|c|c|}
\hline \multirow{2}{*}{$\begin{array}{l}\mathrm{JOH} \\
\mathrm{JOH}\end{array}$} & 21255.00 & OFF！ PRESSURIZED INSECT REPELLENT \\
\hline & 21299.00 & OFF! SKINTASTIC LOTION INSECT REPELLENT (WITH \\
\hline JOL & 19457.00 & BITE FREE INSECT REPELLENT \\
\hline JOL & 20710.00 & SUREKILLER BITE FREE II INSECT REPELLENT \\
\hline KSL & 18958.00 & KELSEY TRAIL BUG BUSTER INSECT REPELLENT JACKET \\
\hline KSL & 18959.00 & KELSEY TRAIL BUG BUSTER INSECT REPELLENT \\
\hline LAL & 18421.00 & PROTECTION INSECT REPELLENT \\
\hline MEI & 16486.00 & STOP BITE INSECT REPELLENT LIQUID \\
\hline MLS & 18364.00 & CUTTER EVERGREEN SCENT INSECT REPELLENT \\
\hline MLS & 18365.00 & CUTTER INSECT REPELLENT \\
\hline MLS & 18366.00 & CUTTER EVERGREEN SCENT INSECT REPELLENT CREAM \\
\hline MLS & 18367.00 & CUTTER INSECT REPELLENT CREAM \\
\hline MLS & 19322.00 & CUTTER MAXIMUM STRENGTH INSECT REPELLENT LIQUID \\
\hline NAC & 11140.00 & SWAT SPRAY INSECT REPELLENT \\
\hline NOZ & 12401.00 & NOXEMA INSECT REPELLENT LOTION \\
\hline NOZ & 12402.00 & NOXEMA TAN \& GUARD LOTION \\
\hline PGH & 18093.00 & THE ORIGINAL MUSKOL INSECT REPELLENT \\
\hline PGH & 18778.00 & MUSKOL INSECT REPELLENT WITH SUNSCREEN \\
\hline PGH & 19909.00 & MUSKOL INSECT REPELLENT SPRAY PRESSURIZED \\
\hline PGH & 20455.00 & MUSKOL LITE INSECT REPELLENT SPRAY \\
\hline PIC & 14674.00 & PIC INSECT REPELLENT LOTION \\
\hline PIC & 18105.00 & PIC 75 DEET INSECT REPELLENT \\
\hline PIC & 18543.00 & PIC $X-100$ DEET \\
\hline PUG & 17435.00 & PARASECT INSECT REPELLENT \\
\hline RAW & 14153.00 & RAWLEIGH INSECT REPELLENT \\
\hline REC & 7137.00 & NERO INSECT REPELLENT SOLUTION \\
\hline REC & 11219.00 & "Z" INSECT REPELLENT SOLUTION \\
\hline REC & 11430.00 & CANADIAN TIRE INSECT REPELLENT \\
\hline REC & 15351.00 & RECORD 100 INSECT REPELLENT \\
\hline SAF & 16056.00 & SANEX JUNGLE POWER INSECT REPELLENT \\
\hline SAF & 17341.00 & JUNGLE POWER INSECT REPELLENT \\
\hline SAF & 18153.00 & JUNGLE POWER FOAM INSECT REPELLENT \\
\hline SAF & 18707.00 & $\begin{array}{l}\text { SANEX JUNGLE POWER PRESSURIZED SPRAY INSECT } \\
\text { REPELLENT }\end{array}$ \\
\hline SAF & 19607.00 & VET-TEK MUSTANG INSECT REPELLENT \\
\hline SAN & 11309.00 & SANFAX BUG OFF INSECT REPELLENT \\
\hline SEM & 18399.01 & HURK III INSECT REPELLENT \\
\hline SIH & 19910.00 & BUZAWAY INSECT REPELLENT SPRAY \\
\hline STL & 14292.00 & 6-12 PLUS INSECT REPELLENT STICK \\
\hline STL & 14293.00 & 6-12 PLUS INSECT REPELLENT SPRAY \\
\hline STL & 14294.00 & 6-12 PLUS INSECT REPELLENT LOTION \\
\hline STO & 14316.00 & REPEX INSECT REPELLENT \\
\hline STO & 20489.00 & REPEX INSECT REPELLENT PRESSURIZED SPRAY \\
\hline STT & 16970.00 & LIQUID INSECT REPELLENT NO 955 \\
\hline TET & 18326.00 & BLAST INSECT REPELLENT \\
\hline TNR & 20375.00 & BEN'S 100 SPRAY INSECT REPELLENT \\
\hline TNR & 20376.00 & BEN'S 100 LOTION INSECT REPELLENT \\
\hline
\end{tabular}




$\begin{array}{ll}\text { TRO } & 11841.00 \\ \text { TRO } & 18398.00 \\ \text { WAL } & 14326.00 \\ \text { WDG } & 19412.00 \\ \text { WES } & 18310.00 \\ \text { WHS } & 18399.02 \\ \text { WIC } & 20018.00 \\ \text { WIC } & 20392.00 \\ \text { WIS } & 17238.00 \\ \text { WIS } & 17239.00 \\ \text { WIS } & 18148.00 \\ \text { WIS } & 18194.00 \\ \text { WIS } & 18195.00 \\ \text { ZOD } & 16861.00 \\ \text { ZOD } & 20188.00\end{array}$

ETHYLHEXANEDIOL
TROJAN TRO-PELL TRL-455

TROJAN CHEMICALS TRB-505 INSECT REPELLENT WATKINS INSECT REPELLENT LOTION WEDGCO BUZZ-OFF WILD ONE INSECT REPELLENT INSECT REPELLENT

WIN INSECT REPELLENT II WIN PUMP INSECT REPELLENT IB REPEL 100 INSECT REPELLENT LIQUID REPEL INSECT REPELLENT LOTION REPEL INSECT REPELLENT PRESSURIZED SPRAY REPEL INSECT REPELLENT PUMP SPRAY REPEL 100 INSECT REPELLENT NON-AEROSOL PUMP ZOECON VAPORETTE REPEL 30 REPELLENT STARBAR EQUINE SUPER WIPE

CHASSE INSECTES INSECT REPELLENT

INLAND-ALCARE ELLIS INSECT REPELLENT

BF-100 BLACK FLY REPELLENT

$X$-IT INSECT REPELLENT

$X$-IT INSECT REPELLENT STICK

6-12 PLUS INSECT REPELLENT STICK

6-12 PLUS INSECT REPELLENT SPRAY

6-12 PLUS INSECT REPELLENT LOTION

VAPORETTE REPEL PERSONAL INSECT REPELLENT

OIL OF LAVENDER

PEV $9930.00 \quad$ FLY SCREEN

BISBUTYLENE TETRAHYDROFURFURAL

$\begin{array}{lr}\text { AIG } & 14333.00 \\ \text { AVM } & 18312.00 \\ \text { BAX } & 10862.00 \\ \text { BAX } & 19659.00 \\ \text { BDC } & 18399.00 \\ \text { BDC } & 20837.00 \\ \text { BOY } & 17956.00 \\ \text { CCN } & 20498.00 \\ \text { CCN } & 20774.00 \\ \text { CCN } & 20817.00 \\ \text { CGC } & 4039.00 \\ \text { CGC } & 8679.00 \\ \text { CGC } & 12958.00 \\ \text { COS } & 18400.00\end{array}$

AIR GUARD INSECT REPELLENT BUSH STRENGTH ATTACK INSECT REPELLENT

PARA - S BOMB

SPRAY N' REPEL

BRENTDALE CHEMICALS INSECT REPELLENT

INSECT REPELLENT

COMBAT INSECT REPELLENT

CCL INSECT REPELLENT III

CCL QUICK-BREAKING INSECT REPELLENT FOAM II

CCL PUMP INSECTICIDE SPRAY VII

TANTOO REPELLENT

TANTOO LIQ INSECT REPELLENT

TANTOO GEL

COPELAND INSECT REPELLENT PRESSURIZED SPRAY 


\begin{tabular}{|c|c|c|}
\hline FAR & 13923.00 & SUPER SWAT FLY REPELLENT \\
\hline FFL & 19522.00 & BUG STOP 50 \\
\hline FUB & 10790.00 & FULLER REPEL-GEL \\
\hline GUC & 18199.00 & GOTCHA! INSECT REPELLENT \\
\hline HAU & 15576.00 & HARTZ DOG FLEA \& TICK SPRAY \\
\hline HAU & 15577.00 & HARTZ CAT FLEA \& TICK SPRAY \\
\hline INT & 9601.00 & CO-OP INSECT REPELLENT \\
\hline INT & 19772.00 & CO-OP INSECT REPELLENT PRESSURIZED SPRAY \\
\hline JAN & 18389.00 & $\begin{array}{l}\text { SIPHEX } 14 \text { INSECTICIDE WITH REPELLENT (FOR CATS } \\
\& \text { DOGS) }\end{array}$ \\
\hline $\mathrm{JOH}$ & 13252.00 & DEEP WOODS FORMULA OFF INSECT REPELLENT LOTION \\
\hline $\mathrm{JOH}$ & 13253.00 & DEEP WOODS PRESSURIZED SPRAY INSECT REPELLENT \\
\hline $\mathrm{JOH}$ & 19730.00 & DEEP WOODS PUMP SPRAY INSECT REPELLENT \\
\hline $\mathrm{JOH}$ & 21299.00 & $\begin{array}{l}\text { OFF! SKINTASTIC LOTION INSECT REPELLENT (WITH } \\
\text { ALOE VERA) }\end{array}$ \\
\hline JOL & 19457.00 & BITE FREE INSECT REPELLENT \\
\hline JOL & 20710.00 & SUREKILLER BITE FREE II INSECT REPELLENT \\
\hline NAC & 11140.00 & SWAT SPRAY INSECT REPELLENT \\
\hline PIC & 14674.00 & PIC INSECT REPELLENT LOTION \\
\hline SAF & 18153.00 & JUNGLE POWER FOAM INSECT REPELLENT \\
\hline SAF & 19607.00 & VET-TEK MUSTANG INSECT REPELLENT \\
\hline SAN & 11309.00 & SANFAX BUG OFF INSECT REPELLENT \\
\hline SEM & 18399.01 & HURK III INSECT REPELLENT \\
\hline STO & 18041.00 & REPEX INSECT REPELLENT \\
\hline TRO & 18398.00 & TROJAN CHEMICALS TRB-505 INSECT REPELLENT \\
\hline WDG & 19412.00 & WEDGCO BUZZ-OFF \\
\hline WES & 18310.00 & WILD ONE INSECT REPELLENT \\
\hline WHS & 18399.02 & INSECT REPELLENT \\
\hline WIC & 20018.00 & WIN INSECT REPELLENT II \\
\hline WIC & 20392.00 & WIN PUMP INSECT REPELLENT IB \\
\hline ZOD & 20188.00 & STARBAR EQUINE SUPER WIPE \\
\hline \multicolumn{3}{|c|}{ DI-N-PROPYL ISOCINCHOMERONATE } \\
\hline AFL & 16041.01 & DELLA DAIRY SPRAY \\
\hline AIG & 14333.00 & AIR GUARD INSECT REPELLENT BUSH STRENGTH \\
\hline AVM & 18312.00 & ATTACK INSECT REPELLENT \\
\hline BDC & 18399.00 & BRENTDALE CHEMICALS INSECT REPELLENT \\
\hline BDC & 20837.00 & INSECT REPELLENT \\
\hline BOY & 17956.00 & COMBAT INSECT REPELLENT \\
\hline $\mathrm{CCN}$ & 20498.00 & CCL INSECT REPELLENT III \\
\hline CGC & 4039.00 & TANTOO REPELLENT \\
\hline CGC & 12120.00 & CIBA-GEIGY PREMIUM LIVESTOCK SPRAY LIQUID \\
\hline CGC & 12958.00 & TANTOO GEL \\
\hline CLB & 20534.00 & PURGE FOAM INSECT REPELLENT FOR HORSES \\
\hline $\cos$ & 18400.00 & COPELAND INSECT REPELLENT PRESSURIZED SPRAY \\
\hline EMO & 18587.00 & BANISH \\
\hline EMO & 18588.00 & SWAT \\
\hline
\end{tabular}




$\begin{array}{lrl}\text { EMO } & 18589.00 & \text { HOG -WASH } \\ \text { EMO } & 18590.00 & \text { EVICT } \\ \text { FAR } & 10375.00 & \text { FARNAM WIPE LIQ WIPE-ON FLY REPELLENT } \\ \text { FAR } & 13438.00 & \text { ROLL-ON FLY REPELLENT \& INSECTICIDE } \\ \text { FAR } & 13554.00 & \text { FARNAM FLYS - AWAY REPELLENT BOMB II } \\ \text { FAR } & 13922.00 & \text { SWAT FLY REPELLENT CREAM } \\ \text { FFL } & 19522.00 & \text { BUG STOP 50 } \\ \text { FUB } & 10790.00 & \text { FULLER REPEL-GEL } \\ \text { GUC } & 18199.00 & \text { GOTCHA! INSECT REPELLENT } \\ \text { INT } & 9601.00 & \text { CO-OP INSECT REPELLENT } \\ \text { INT } & 19735.00 & \text { CO-OP HORSE GARD INSECTICIDE REPELLENT } \\ \text { INT } & 19772.00 & \text { CO-OP INSECT REPELLENT PRESSURIZED SPRAY } \\ \text { JOH } & 13253.00 & \text { DEEP WOODS PRESSURIZED SPRAY INSECT REPELLENT } \\ \text { JOL } & 19457.00 & \text { BITE FREE INSECT REPELLENT } \\ \text { JOL } & 20710.00 & \text { SUREKILLER BITE FREE II INSECT REPELLENT } \\ \text { KEK } & 18583.00 & \text { BODY GARD } \\ \text { KEK } & 18584.00 & \text { HORSE-SHOO } \\ \text { LOR } & 12585.00 & \text { LORRAIN RUB-ON LIQUID HORSE INSECTICIDE } \\ \text { MLS } & 18365.00 & \text { CUTTER INSECT REPELLENT } \\ \text { MLS } & 18366.00 & \text { CUTTER EVERGREEN SCENT INSECT REPELLENT CREAM } \\ \text { MLS } & 18367.00 & \text { CUTTER INSECT REPELLENT CREAM } \\ \text { NAC } & 11140.00 & \text { SWAT SPRAY INSECT REPELLENT } \\ \text { PGH } & 18093.00 & \text { THE ORIGINAL MUSKOL INSECT REPELLENT } \\ \text { PGH } & 18778.00 & \text { MUSKOL INSECT REPELLENT WITH SUNSCREEN } \\ \text { PGH } & 19909.00 & \text { MUSKOL INSECT REPELLENT SPRAY PRESSURIZED } \\ \text { PGH } & 20455.00 & \text { MUSKOL LITE INSECT REPELLENT SPRAY } \\ \text { PIC } & 14674.00 & \text { PIC INSECT REPELLENT LOTION } \\ \text { RAL } & 10434.00 & \text { PURINA RUB-ON EMULSION HORSE INSECTICIDE } \\ \text { SAF } & 18153.00 & \text { JUNGLE POWER FOAM INSECT REPELLENT } \\ \text { SAF } & 18707.00 & \text { SANEX JUNGLE POWER PRESSURIZED SPRAY INSECT } \\ \text { SAF } & 19607.00 & \text { VET-TEK MUSTANG INSECT REPELLENT } \\ \text { SAN } & 11309.00 & \text { SANFAX BUG OFF INSECT REPELLENT } \\ \text { SEM } & 18399.01 & \text { HURK III INSECT REPELLENT } \\ \text { STO } & 18041.00 & \text { REPEX INSECT REPELLENT } \\ \text { TRO } & 18398.00 & \text { TROJAN CHEMICALS TRB - 505 INSECT REPELLENT } \\ \text { WDG } & 19412.00 & \text { WEDGCO BUZZ-OFF } \\ \text { WES } & 18310.00 & \text { WILD ONE INSECT REPELLENT } \\ \text { WHS } & 18399.02 & \text { INSECT REPELLENT } \\ \text { WIC } & 20392.00 & \text { WIN PUMP INSECT REPELLENT IB } \\ \text { ZOD } & 16041.00 & \text { STARBAR DAIRY SPRAY WITH REPELLENT } \\ \text { ZOD } & 20188.00 & \text { STARBAR EQUINE SUPER WIPE } \\ & & \end{array}$

\section{N-OCTYL BICYCLOHEPTENE DICARBOXIMIDE}

$\begin{array}{lll}\text { AIG } & 14333.00 & \text { AIR GUARD INSECT REPELLENT BUSH STRENGTH } \\ \text { AVM } & 18312.00 & \text { ATTACK INSECT REPELLENT } \\ \text { BAX } & 19659.00 & \text { SPRAY N }\end{array}$




\begin{tabular}{|c|c|c|}
\hline BDC & 18399.00 & BRENTDALE CHEMICALS INSECT REPELLENT \\
\hline BOY & 17956.00 & COMBAT INSECT REPELLENT \\
\hline $\mathrm{CCN}$ & 20774.00 & CCL QUICK-BREAKING INSECT REPELLENT FOAM II \\
\hline $\mathrm{CCN}$ & 20817.00 & CCL PUMP INSECTICIDE SPRAY VII \\
\hline CGC & 4039.00 & TANTOO REPELLENT \\
\hline CGC & 8679.00 & TANTOO LIQUID INSECT REPELLENT \\
\hline CGC & 12958.00 & TANTOO GEL \\
\hline CLB & 20534.00 & PURGE FOAM INSECT REPELLENT FOR HORSES \\
\hline $\cos$ & 18400.00 & COPELAND INSECT REPELLENT PRESSURIZED SPRAY \\
\hline EMO & 18587.00 & BANISH \\
\hline EMO & 18588.00 & SWAT \\
\hline EMO & 18589.00 & HOG-WASH \\
\hline EMO & 18590.00 & EVICT \\
\hline FAR & 13438.00 & ROLL-ON FLY REPELLENT \& INSECTICIDE \\
\hline FAR & 13923.00 & SUPER SWAT FLY REPELLENT \\
\hline FFL & 19522.00 & BUG STOP 50 \\
\hline FUB & 10790.00 & FULLER REPEL-GEL \\
\hline GUC & 18199.00 & GOTCHA！ INSECT REPELLENT \\
\hline $\mathrm{HAU}$ & 15576.00 & HARTZ DOG FLEA \& TICK SPRAY \\
\hline $\mathrm{HAU}$ & 15577.00 & HARTZ CAT FLEA \& TICK SPRAY \\
\hline INT & 19735.00 & CO-OP HORSE GARD INSECTICIDE REPELLENT \\
\hline JAN & 18389.00 & $\begin{array}{l}\text { SIPHEX } 14 \text { INSECTICIDE WITH REPELLENT (FOR CATS } \\
\& \text { DOGS) }\end{array}$ \\
\hline $\mathrm{JOH}$ & 13252.00 & DEEP WOODS FORMULA OFF INSECT REPELLENT LOTION \\
\hline $\mathrm{JOH}$ & 19730.00 & DEEP WOODS PUMP SPRAY INSECT REPELLENT \\
\hline JOL & 19457.00 & BITE FREE INSECT REPELLENT \\
\hline JOL & 20710.00 & SUREKILLER BITE FREE II INSECT REPELLENT \\
\hline KEK & 18583.00 & BODY GARD \\
\hline KEK & 18584.00 & HORSE - SHOO \\
\hline LOR & 12585.00 & LORRAIN RUB-ON LIQUID HORSE INSECTICIDE \\
\hline MLS & 18364.00 & CUTTER EVERGREEN SCENT INSECT REPELLENT \\
\hline MLS & 18366.00 & CUTTER EVERGREEN SCENT INSECT REPELLENT CREAM \\
\hline MLS & 18367.00 & CUTTER INSECT REPELLENT CREAM \\
\hline NAC & 11140.00 & SWAT SPRAY INSECT REPELLENT \\
\hline PGH & 18093.00 & THE ORIGINAL MUSKOL INSECT REPELLENT \\
\hline PGH & 18778.00 & MUSKOL INSECT REPELLENT WITH SUNSCREEN \\
\hline PGH & 20455.00 & MUSKOL LITE INSECT REPELLENT SPRAY \\
\hline PIC & 14674.00 & PIC INSECT REPELLENT LOTION \\
\hline RAL & 10434.00 & PURINA RUB-ON EMULSION HORSE INSECTICIDE \\
\hline SAF & 18707.00 & $\begin{array}{l}\text { SANEX JUNGLE POWER PRESSURIZED SPRAY INSECT } \\
\text { REPELLENT }\end{array}$ \\
\hline SAF & 19607.00 & VET-TEK MUSTANG INSECT REPELLENT \\
\hline SAN & 11309.00 & SANFAX BUG OFF INSECT REPELLENT \\
\hline SEM & 18399.01 & HURK III INSECT REPELLENT \\
\hline STO & 18041.00 & REPEX INSECT REPELLENT \\
\hline TRO & 18398.00 & TROJAN CHEMICALS TRB-505 INSECT REPELLENT \\
\hline WDG & 19412.00 & WEDGCO BUZZ-OFF \\
\hline WES & 18310.00 & WILD ONE INSECT REPELLENT \\
\hline
\end{tabular}




\section{APPENDIX II}

\section{SPRAY EQUIPMENT USED IN CANADA FOR MOSQUITO CONTROL}

A wide variety of insecticide application equipment is used in mosquito control programs in Canada. Sprayers can be conveniently divided into equipment used for mosquito larviciding and for mosquito adulticiding. Some equipment may be used for either method of mosquito control (e.g., back-pack sprayers); other equipment may be used only for adulticiding (e.g., ULV generators).

Although specific examples of spray equipment are given below to illustrate the choices available, mention of a particular product does not constitute endorsement. Other products, equivalent to those mentioned, may be available locally and perform equally well.

Because most of the spray equipment used in Canada is manufactured in the U.S.A., tank sizes are given in U.S. gallons.

\section{Mosquito Larviciding}

\section{(1) Portable, Manual}

(a) Hand-carried, liquid sprayer

The standard, two- or three-gallon, pressurized-type, sprayer is often used to apply a larvicide to small-sized mosquito breeding sites. These simple sprayers (equipped with a stainless steel tank, an 8-inch gun valve unit and wand, 4 feet of hose, and an adjustable nozzle tip assembly) can provide many years of service. An example is the B \& G Extenda-Ban Model N-224S.

(b) Neck-carried, granular applicator

Sometimes referred to as "belly grinders", these units are bucket-shaped, have a rotary hand-crank for auguring granules through a bottom-mounted granule spreader, and are hung from the operator's neck.

Most such spreaders are used to apply fertilizer or herbicide granules to home grounds. Although not used commonly for mosquito larviciding (because of the discomfort of the unit, the weight of granules, and its dust-generating properties), they can be useful when better granular application equipment is unavailable. A variety of makes and models are available at garden supply centres.

(c) Back-Pack, liquid sprayer 
Many brands of these multi-purpose sprayers are available. They are used to apply fertilizers, pesticides, spray water onto small fires, etc. Typically, a plastic, $20 \mathrm{~L}$, tank is mounted to a back-pack harness and a side-mounted hand-pump lever is used to pressurize the tank, moving the insecticide through a 3 or 4 foot hose and the gun valve unit and wand. For larviciding, a coarse, pin-stream nozzle is most often used. An example is the CP3 Mk2.

\section{(2) Portable, Powered}

(a) Back-Pack, liquid sprayer

These units are powered by a small, gasoline engine. The tank is moulded above the engine and both are mounted to a backpack carrier. Some units only dispense liquid sprays; others, depending on options, can dispense sprays as a mist and/or dispense granules. Although some units are very noisy and heavy, they are an efficient, low-cost means of spraying. An example of this type of sprayer is the Echo SHR-200E.

A multi-mode example is the Echo Model DM-9. This versatile unit is a combination sprayer and duster. It can dispense a liquid spray, mist, dust or granules depending on the configuration of options used. In mosquito control work, it is used most often as either a mist-blower or as a granular applicator.

(b) Back-pack, mist-blower

These units are equipped with a special misting nozzle.

These units can mist up to $10 \mathrm{~m}$ from the blower. See also discussion in 2 (a) above. An example is the Echo Model DM-9, described above.

(c) Back-pack, granular applicator:

The unit is equipped with a granule spreading nozzle. Granules can be spread up to 8-10 m from the unit, depending on nozzle angle and winds. See also discussion in 2 (a) above. An example is the Echo Model DM-9, described above.

\section{(3) Truck-mounted, powered}

(a) Liquid, hose and nozzle sprayer

Because these types of sprayers are commonly used by commercial applicators and farmers to apply pesticides and fertilizers, there are countless makes and models available.

One example is the Agrotec which, like many other such skidmounted units, comes with a 100, 150, or 200 gallon tank and a gun, hose and reel kit.

(b) Liquid, mist-blower 
Mist-blowers used for tree spraying also can be used to apply a residual spray to mosquito resting and oviposition sites. A popular unit amongst mosquito abatement workers is the heavyduty, mister-duster combination sprayer. These units have a liquid tank (or a 45-gallon drum) and a granule hopper. Thus, they can be used to apply liquid mists or granules. One such unit is the Buffalo Turbine Sprayer-Duster Model K.

(c) Granular-blower applicator. See (3)(c) above.

(4) Trailer-mounted, powered:

(a) Liquid, mist-blower

These sprayers are typically used for spraying large trees to control defoliating insects but are also used by some municipalities to apply residual sprays to wide, roadside ditches to kill resting, adult mosquitoes. They are not suitable for small areas or narrow ditches. An example is the John Bean mistblower or tree sprayer. Several models are used.

\section{(5) Aircraft-mounted, powered}

(a) Boom and nozzle sprayer

Boom and nozzle liquid sprayers are not used commonly for mosquito larviciding because of problems associated with spray evaporation, drift and foliage penetration and difficult terrain. However, they are sometimes used to apply 'liquid larvicides to wide, drainage ditches, sewage lagoons and sloughs, using aircraft.

Most equipment used on agricultural fixed-and rotary-wing aircraft is suitable. A commonly used brand is Simplex gear.

(b) Granular applicator

Granular larvicides are frequently applied by aircraft (mostly rotary-wing) to mosquito larval breeding sites. Canada.

Simplex gear is probably the most often equipment used in

\section{Mosquito Adulticiding}

\section{(1) Portable, Powered}

(a) Hand-carried, liquid sprayer

Sma11, hand-carried, pressurized are not widely used for adult mosquito control. Their use is limited to applying a residual spray to walls of buildings (e.g., stables, homes), near doors and windows, where adult mosquitoes may rest or attempt to enter a structure. An example is the B \& G Extenda-Ban Model N- 
(b) Hand-carried, thermal fogger

These foggers thermal-pneumatically produce aerosol droplets 10-60 microns in diameter. Most units have a 5-7 L tank for the fogging solution (usually a mixture of diesel and insecticide concentrate). This type of aerosol generator uses relatively old technology and most mosquito abatement agencies have or are switching over to non-thermal aerosol generators (see below). Portable, propane, thermal foggers generally are not suitable for mosquito control. An example is the Swingfog Model SN 50.

(c) Hand-carried, ULV generator

Several light-weight, gasoline-powered units are available and suitable for smaller outdoor mosquito adulticiding. They have a limited tank capacity (usually 0.25-0.5 gallons). These units are popular for use around farms, golf courses, small parks and campgrounds. Care should be taken to select a unit that will stand up to normal use. The most commonly used unit in Canada is the Leco ULV P-1.

(d) Back-Pack, liquid sprayer

These units, equipped with a flat-fan nozzle, may be used to apply a residual spray around buildings and vegetation to kill resting mosquitoes. An example of the liquid only sprayer is the Echo SHR-200E.

(e) Back-pack, mist-blower

These units are much more efficient than the liquid sprayers above. They can treat larger mosquito resting sites must faster because of their greater swath width and powered, pumping system. An example is the Echo Model DM-9.

\section{(2) Truck-mounted, powered}

(a) Liquid, hose and nozzle sprayer

As noted above, these types of sprayers are commonly used by commercial applicators and farmers to apply pesticides and fertilizers. As a result, there are countless makes and models available. Gasoline- or electric-powered units can be purchased or assembled locally from readily available components.

One example is the Agrotec which, like many other such skidmounted units, comes with a 100, 150, or 200 gallon tank and a gun, hose and reel kit.

(b) Liquid, mist-blower

Most such units have been developed for urban forestry and orchard use. Municipalities responsible for both mosquito and 
tree pest management use these sprayers for both purposes. The heavy-duty units can apply a wide swath of mist of residual mosquito adulticide through mosquito resting and oviposition sites (e.g., wide, grassy, roadside ditches). An example of this type of mist-blower is the Buffalo Turbine Sprayer-Duster Model $\mathrm{K}$.

\section{(c) Thermal fogger}

Based on the same principle as the hand-carried thermal foggers, these units thermally produce aerosol droplets 5-200 microns in diameter. Heavy-duty, skid-mounted, models are usually used in routine mosquito fogging programs. As noted above, most mosquito abatement agencies have replaced their old thermal foggers with the more modern cold aerosol equipment. An example is the TIFA Model 100-E.

\section{(d) ULV generator}

Many different kinds of light-, medium- and heavy-duty ULV generators are available for skid- or fixed-mounting on allterrain vehicles, trucks and trailers. Commonly-used brands include those of Leco, Londonaire, Microgen and Curtis-Dyna. Most units draw the adulticide from the original, unpressurized $20 \mathrm{~L}$ insecticide container. One of the most commonly-used, heavyduty, gasoline-powered, skid-mounted units for mosquito adulticiding is the Leco Model HD.

A relatively-new, battery-operated ULV generator is the Whispermist (Models 10 and 20). This type of cold fogger can be powered using a separate 12 volt battery or the truck battery. It is a light-weight, medium duty model which has the advantage of being very quiet running.

\section{(3) Aircraft-mounted, powered}

(a) Boom and nozzle sprayer

Conventional boom and nozzle sprayers, like those used for agricultural applications, can also be used to apply residual sprays for adult mosquito control. Several models are available from Simplex.

\section{(b) ULV generator}

ULV nozzles can be substituted for convention teejet nozzles to enable ULV applications of mosquito adulticides. Various types of such rotary atomizers are available. They may be driven by wind, hydraulics or electricity. Beecomist Model 360 sprayheads have been used successfully on both rotary- and fixed-wing aircraft. 
SUPPLIERS OF REPELLENTS AND INSECTICIDES COMMONLY USED FOR MOSQUITO CONTROL IN CANADA

ABE ABELL WACO LTD., 246 ATWELL DR., REXDALE, ON, M9W 5 B4. $416-675-1635$

ABT ABBOTT LABORATORIES, CAPD D-928, NORTH CHICAGO, IL, 60064, USA.

$312-937-8904$

AFL ALFA-LAVAL AGRI, 2020 FISHER DR., PETERBOROUGH, ON, K9J $7 \mathrm{~B} 7$. $705-876-3122$

AFY

ALL FOR YOU PRODUCTS INC., BOX 179, SMEATON, SK, SOJ 2JO. $306-426-2031$

AIG AIR GUARD CONTROL INC., 26 WATERMAN AVE., TORONTO, ON, M4B $1 Y 5$.

$416-499-8500$

ALT

ALSI CIE LTEE, 150 RUE SEIGNEURIALE, C.P. 5040, BEAUPORT, - PQ, G1E 4Y6. $418-661-6703$

AMW AMWAY CORP., 7575 E. FULTON RD., ADA, MI, 49355, USA. $616-676-5428$

ARE

ARCTIC TRADING CO. INC., P.O. BOX 910, CHURCHILL, MB, ROB OEO. $204-675-2164$

ARI AMERICAN REPELLENT INC., 1200 JAMES RD., N. LITTLE ROCK, AR, 72118, USA. $501-851-2337$

AVM AVMOR LTD., 433 RUE STE-HELENE, MONTREAL, PQ, H2Y 2L1. $514-849-4541$

BAX BAYVET DIV. CHEMAGRO LTD., 77 BELFIELD RD., ETOBICOKE, ON, M9W 1 G6.

$416-248-0771$

BDC

BRENTDALE CHEMICALS, 41 RACINE RD., REXDALE, ON, M9W 226. 
BOY BOYLE-MIDWAY CANADA LTD., 2 WICKMAN RD., TORONTO, ON, M8Z $5 \mathrm{M} 5$.

$416-255-9163$

CCN CCL INDUSTRIES, 26 WATERMAN AVE., TORONTO, ON, M4B 1 Y5. $416-755-9271$

CGC CIBA-GEIGY CAN/AG DIV., 6860 CENTURY AVE., MISSISSAUGA, ON, L5N 2 W5.

$416-821-4420$

CHH CHEMAGRO LTD., 256 BRITANNIA RD.E., MISSISSAUGA, ON, L4Z $1 S 6$.

$416-890-0332$

CHP CHIPMAN INC., 400 JONES RD., P.O. BOX 9910, STONEY CREEK, ON, L8G $3 \mathrm{Z1}$.

$416-643-4123$

CHZ COGHLAN'S LTD., 121 IRENE ST., WINNIPEG, MB, R3T 4C7. $204-284-9550$

CLB CLINE BUCKNER INC., SUBSIDIARY WATERBURY INC. CO., P.O.BOX 640,100

CALHOUN ST., INDEPENDENCE, LA, 70443, USA.

$504-878-6751$

COS COPELAND LABORATORIES LTD., 41 RACINE RD., REXDALE, ON, M9W $2 Z 6$.

$416-743-4272$

CYC CYANAMID CANADA INC., 88 MCNABB ST., MARKHAM, ON, L3R 6E6. $416-470-3600$

DEV DECA INVESTMENTS, P.O. BOX 1, KIRKLAND LAKE, ON, P2N 3M6. $705-567-7844$

DFT DRAFT ENTERPRISES LTD., 912 STRATHCONA RD., EAST SELKIRK, MB, ROE OMO.

$204-738-2656$

DIS PRODUITS VETERINAIRES DISPAR, 675 ST-PIERRE SUD, JOLIETTE, $P Q$, J6E

321 .

$514-759-0497$

DIT DITCHLING CORP. LTD., P.O. BOX 395, DON MILLS, ON, M3C 
257.

$416-438-6807$

DOW DOW CHEMICAL OF CANADA LTD., P.O. BOX 1012, MODELAND RD., SARNIA, ON, N7T 7K7.

$519-339-5029$

DTC DRUG TRADING CO. LTD., 1960 EGLINTON AVE. E., BOX 335, STATION "A", SCARBOROUGH, ON, M1K 5C1. 416-288-1100

DUP DUPHAR B.V., C.J. VAN HOUTENLAAN 36, WEESP, 1381 CP, THE NETHERLANDS

EMO EMPIRE INTERNATIONAL, P.O. BOX 695, STREETSVILLE POSTAL STATION, MISSISSAUGA, ON, L5M 2C2

FAR FARNAM COMPANIES, 301 WEST OSBORN RD., PHOENIX, AZ, 85013-3928, USA. $602-285-1660$

FFL FLORA \& FAUNA LABS. INC., 8500 PILLSBURY AVE. SO., MINNEAPOLIS, MN, 55420, USA.

$612-881-6908$

FUB FULLER BRUSH CO., 1115 GUELPH LINE, P.O. BOX 5019, BURLINGTON, ON, L7R $3 Z 8$.

416-335-8000

GAX GARDEX CHEMICALS LTD., 246 ATTWELL DR., REXDALE, ON, M9W $5 \mathrm{~B} 4$.

$416-6751638$

GRA GREENLEAF GARDEN SUPPLIES, 4612 DAWSON ST., P.O. BOX 82338 , BURNABY, BC, V5C 5 P8. 604-299-9505

GUC GUARDIAN CHEMICALS, P.O. BOX 3029, FORT SASKATCHEWAN, AB, T8L 2 T1.

HAU HARTZ CANADA INC., 1125 TALBOT ST., ST. THOMAS, ON, N5P 3W7.

$519-631-7660$

HOH HOME HARDWARE STORES LTD., 34 HENRY ST. W., ST. JACOBS, ON, NOB 2NO.

$519-664-2252$ 
ILD INLAND ALCARE JANITOR SUPPLIES, 10916-119 ST., EDMONTON, $A B, T 5 H 3 P 4$.

$403-453-5800$

INT INTERPROVINCIAL CO-OP LTD., 945 MARION ST., ST. BONIFACE, MB, R2J OK7.

$204-233-3461$

JAN JANSSEN PHARMACEUTICA, ANIMAL HEALTH DIV., 1-6705

MILLCREEK DR., MISSISSAUGA, ON, L5N 5R9.

$416-567-2504$

JBL LJB LABORATORIES, 1001 E. CASS ST., ST. JOHNS, MI, 48879, USA.

$517-224-4784$

JOH JOHNSON (S.C.) \& SON LTD., 1 WEBSTER ST., BOX 520, BRANTFORD, ON, N3T 5R1.

$519-756-7900$

JOL JOHN LIM CO.(THE), 1285 ST. MARY'S AVE., MISSISSAUGA, ON, L5E 1 M8.

$416-271-2711$

KEK KEM MANUFACTURING CAN. LTD., 6660 CAMPOBELLO RD., MISSISSAUGA, ON, L5N 2 L9.

416-826- 8240

KEM KEMSAN INC., 462 TRAFALGAR RD.BOX 727, OAKVILLE, ON, L6J $5 \mathrm{C} 1$.

$416-8452271$

KSL KELSEY SPORTSWEAR LTD., 563 NOTRE DAME AVE., WINNIPEG, MB, R3B $1 S 5$.

204-786-1503

LAL LALCO SUPERCO LTD., C.P.280 STATION ROSEMOUNT, MONTREAL, $\mathrm{PQ}, \mathrm{H} 1 \mathrm{X}$ 3B8.

LAT LATER CHEMICALS LTD., 12080 HORSESHOE WAY, RICHMOND, BC, V7A 4 V5.

$604-271-4224$

LOR LORRAIN, LEO LABS ENGR., 6151 IRWIN ST., LASALLE, PQ, H8N $1 \mathrm{~A} 1$.

$514-366-4805$ 
RHONE POULENC CANADA INC., 2000 ARGENTIA RD., PLAZA 3, SUITE 400, MISSISSAUGA, ON, L5N 1 V9.

$416-821-4450$

MCO MCKIRDY (J.G.M.) LTD., 19 LAKELAND POINT DR., KINGSTON, ON, K7M 4E8.

$613-389-5573$

MEI MEIER, IAAN, 31 EDILOU DR., TORONTO, ON, M8W 4B1

MLS MILES LABORATORIES INC., HOUSEHOLD PRODUCTS DIV., $7123 \mathrm{~W}$. $65 \mathrm{TH}$ ST., CHICAGO, IL, 60638, USA.

$312-458-6100$

MRA MARTIN OUTDOOR PRODUCTS, O.B. FASAN, 169 AURORA HTS DR, AURORA, ON, L4G $2 X 1$.

$416-841-6760$

MFX MORFLEX CHEM. CO., 2110 HIGH POINT RD., GREENSBORO, NC, 27403 , USA.

$919-292-1781$

NAC NATIONAL CHEMSEARCH OF CAN., DIV. OF NCH CANADA INC . 245 ORENDA RD., BRAMALEA, ON, L6T 1 E7.

$416-457-5220$

NEW

NEWVILLE COMPANY INC., P.O. BOX 5637, STATION "A", CALGARY, AB, T2H $1 Y 1$

NOZ NOXELL (CANADA) CORP., 3333 UNITY DR., MISSISSAUGA, ON, L5L 3 T 3 .

$416-828-2500$

PEI ROUSSEL BIO CORP., 170 BEAVERBROOK RD., LINCOLN PARK, NJ, 07035 , USA. $201-628-7200$

PEV PETRUNKA, MARY, 6-2643 EAST ARTHUR ST., THUNDER BAY, ON, P7E 5 P5.

$807-622-4972$

PGH SCHOLL-PLOUGH CANADA INC., 6400 NORTHAM, MISSISSAUGA, ON, L4J $1 \mathrm{~J} 1$.

$416-755-4141$

PIC PIC CORP., 23 S. ESSEX AVE., ORANGE, NJ, 07050, USA. 
PUG INSECTICIDES PUROGUARD LTEE, 264 RUE QUERBES, DORION, PQ, J7V $1 \mathrm{~J} 7$.

$514-455-7402$

RAL RALSTON PURINA CANADA INC., 6151 RUE IRWIN, LASALLE, PQ, H8N 1 Al.

$514-366-2040$

RAW RAWLEIGH, W.T. CO. CAN. LTD., 354 ISABEY, ST. LAURENT, PQ, H4T 1W1.

$514-342-4212$

REC RECOCHEM INC., 131 EAST DR., BRAMPTON, ON, L6T $1 B 5$. 416-791- 1788

SAF SANEX CHEMICALS LTD., 2695 SLOUGH ST., MISSISSAUGA, ON, L4T $1 \mathrm{G} 2$.

$416-677-4890$

SAN SANFAX INDUSTRIES LTD., TRANS CANADA HWY., 1650 SOUTH SERVICE RD., DORVAL, PQ, H9P 1 H9. $514-683-7700$

SEM SEAL CHEMICAL CORP. CANADA LTD, BOX 103, WINNIPEG, MB, R3C $2 \mathrm{G} 1$.

$204-694-2545$

SIH SI-PHARM LTD., 192 FORSYTH RD., NEWMARKET, ON, L3Y 7X9.

STD STANCHEM, A BUSINESS UNIT OF C-I-L INC., ATTN: D.

MACLEAN, 43 JUTLAND RD., TORONTO, ON, M8Z $2 \mathrm{G} 6$. $416-259-8231$

STL STERLING DRUG LTD., YONGE ST. S., AURORA, ON, L4G 3 H6. 416-773-1122

STO STANLEYKEM INC., P.O. BOX 2099, CAMBRIDGE, ON, N3C 2 V6. $519-658-9449$

STT STANALEX DEVELOPMENTS INC., 392 VODDEN ST.E., BRAMPTON, ON, L6V $2 \mathrm{~N} 3$.

TET TENTEL ENTERPRISES, P.O. BOX 156, BARRIE, ON, L4M 4T2. $705-436-4941$ 
TNR TENDER CORP., P.O. BOX 42, INDUSTRIAL PARK, LITTLETON, NH, 03561 , USA.

$603-444-5464$

TRO TROJAN CHEMICALS, DIV. OF VALLEY CAMP LTD., 41 RACINE RD., REXDALE, ON, M9W $2 Z 6$.

416- $741-7372$

UAG UNITED AGRI PRODUCTS, PO BOX 22116, 439 SOVEREIGN RD., LONDON, ON, N6C 4NO.

$519-659-9111$

UCC UNION CARBIDE CANADA LTD., P.O. BOX 700, POINTE-AUX-TREMBL, PQ, H1B 5K8.

$514-645-5311$

WAL WATKINS INC., 30-5 SCURFIELD BLVD.,, WINNIPEG, MB, R3Y $1 \mathrm{G} 3$.

$204-489-1295$

WDG WEDGCO CHEMICAL LTD., 623 HUNTS CRES. N.W., CALGARY, AB, T2K $4 \mathrm{~J} 2$.

$403-291-2441$

WES WESCLEAN NORTHERN SALES LTD., P.O. BOX 1367, HAY RIVER, NT, X1A 2L8.

WHS WHEELS MAINTENANCE PRODUCTS LTD., 60 SIGNET DRIVE, WESTON, ON, M9L 2 Y4.

$416-740-5885$

WIC WIN CHEMICALS \& EQUIPMENT LTD., 1295 EGLINTON AVE E., UNIT \#11, MISSISSAUGA, ON, L4W 3E6.

416-625-9672

WIS WISCONSIN PHARMACAL CO., 2977 HIGHWAY 60, P.O. BOX 198, JACKSON, WI, 53037, USA.

414-677-4121

ZOC ZOECON INDUSTRIES, 12200 DENTON DRIVE, DALLAS, TX, 75234 , USA.

$214-243-2321$

ZOD ZOECON CANADA INC., 3-12 STANLEY COURT, WHITBY, ON, L1N $8 \mathrm{P} 9$.

$416-430-2511$ 
ZOECON CORP., 975 CALIFORNIA AVE., PALO ALTO, CA, 94304, USA.

$415-857-1130$ 
ARBOVIRUS SURVEILLANCE PROGRAMS IN CANADA, $1980^{3}$

Arbovirus surveillance programs entail monitoring of adult populations of vector species and transmission rates (using sentinel flocks or mammals), virus isolations, and diagnosis of amplifying and dead-end host infections.

Arbovirus surveillance programs are presently carried out annually in Manitoba and Saskatchewan [for western equine encephalitis (WEE)], Newfoundland [for eastern equine encephalitis (EEE)] and Ontario [for St. Louis encephalitis (SLE)]. No programs are carried out in Alberta, British Columbia, the Maritimes, Quebec, or the Territories. The lack of surveillance in these regions is partly a result of the lack of human encephalitis cases in recent years. Provincial governments are not convinced of the need for surveillance. In Quebec there is no one in the government who is responsible to take action, should an outbreak occur.

Manitoba

Arbovirus surveillance is coordinated by the Manitoba Arbovirus Surveillance Committee, which was formed in 1976 after the 1975 outbreak of WEE. A surveillance program is carried out annually to predict (and prevent) outbreaks of WEE in the human population. Virus identification and diagnostic services are available at provincial public health and veterinary laboratories.

\section{Saskatchewan}

An annual arbovirus surveillance program is part of a longterm study initiated over 20 years ago. The surveillance program is a joint venture between scientists at the federal Research Station and the University of Saskatchewan (Department of Veterinary Microbiology), who have expertise and facilities to identify arboviruses.

Ontario

Surveillance for St. Louis encephalitis was initiated after outbreaks of the disease in 1975. A reduction in incidence of human cases of the disease since has resulted in reduced support for surveillance. A surveillance program is being carried out through the joint efforts of University of Guelph and the Essex

3 Chance, M. M. 1982. The Canada Biting Fly Centre. A feasibility study of the proposed Canada Biting Fly Centre. Final Rpt. DSS file no.05SU.01837-8-0177. Contract ser. no. ISU7800295. 243pp. 
County Abatement district. Expertise on mosquito-borne arboviruses is also available at Queen's University.

The National Arbovirus Reference Centre (University of Toronto) provides information expertise, reagents, and identification (diagnostic and confirmatory) services on a national basis. It surveys occurrence and activity of arboviruses across the country. The expertise of its personnel is highly regarded by virologists and other experts. The Centre is cooperating in arbovirus surveillance programs in Ontario and Newfoundland.

\section{Other Regions}

The Animal Diseases Research Institute (Agriculture Canada, Ottawa) provides identification (confirmation) services of arbovirus infections of livestock.

Expert virologists with facilities for identifying arboviruses are located in Vancouver (U.B.C.), Edmonton, (University of Alberta, Public Health Laboratory) and Montreal (Institute Armand-Frappier at l'Université de Montréal) and Halifax (Dalhousie University/Izaak Walton Killam Hospital). In Newfoundland, work is carried out in cooperation with the National Arbovirus Reference Centre. Personnel at U.B.C., University of Saskatchewan, and Dalhousie have carried out surveys of arboviruses in the territories, Alberta, Ontario, Quebec and the Maritimes. 


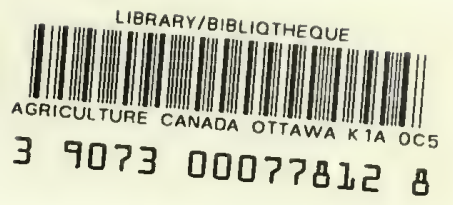


